\title{
Mechanistic and Structural Characterization of Thiamine Diphosphate Dependent Enzyme Transketolases from Human and E.coli
}

\author{
Dissertation \\ for the award of the degree \\ “Doctor rerum naturalium" (Dr.rer.nat.) \\ of the Georg-August-Universität Göttingen
}

within the doctoral program "Basic program. Biology"

of the Georg-August University School of Science (GAUSS)

\author{
submitted by \\ Shao-Bo Dai \\ born in Xi'an, China
}

Göttingen 2017 


\section{Members of the Thesis Committee}

Prof. Dr. Kai Tittmann

Department of Molecular Enzymology

Georg-August University Göttingen

Prof. Dr. Ralf Ficner (First Reviewer)

Department of Molecular and Structural Biology Institut für Mikrobiologie und Genetik Georg-August-Universität Göttingen

Dr. Till Ischebeck (Second Reviewer)

Department of Plant Biochemistry Albrecht-von-Haller-Institut für Pflanzenwissenschaften Georg-August-Universität Göttingen

\section{Further members of the Examination Board}

Prof. Dr. Ivo Feußner

Department of Plant Biochemistry Albrecht-von-Haller-Institut für Pflanzenwissenschaften Georg-August-Universität Göttingen

Prof. Dr. Jörg Stülke

Department of General Microbiology Georg-August-Universität Göttingen

Prof. Dr. Ricardo Mata

Institute of Physical Chemistry Georg-August-Universität Göttingen

Dr. Manfred Konrad

Department of Enzyme Biochemistry Max Planck Institute for Biophysical Chemistry Göttingen Date of the oral examination: 20.06 .2017 
Herewith I declare that I prepared this thesis "Mechanistic and Structural Characterization of Thiamine Diphosphate Dependent Enzyme Transketolases from Human and E.coli" independently and with no other sources and aids than quoted. This thesis (wholly or in part) has not been submitted elsewhere for any academic award or qualification.

Göttingen, 20.04.17

Shao-Bo Dai 


\section{Contents}

List of Figures

$\begin{array}{ll}\text { List of Tables } & 13\end{array}$

$\begin{array}{ll}\text { Abbreviations } & 14\end{array}$

$\begin{array}{ll}\text { Acknowledgements } & 18\end{array}$

$\begin{array}{lll}\text { 1. Introduction } & 19\end{array}$

$\begin{array}{lll}1.1 & \mathbf{1 9}\end{array}$

$\begin{array}{ll}1.2 & \mathbf{2 0}\end{array}$

$\begin{array}{lll}1.3 & \text { Covalent intermediates in ThDP catalysis } & \mathbf{2 4}\end{array}$

1.4 Biological function of ThDP-dependent enzymes and transketolase $\quad 27$

$\begin{array}{lll}1.5 & \text { Catalytic mechanism of transketolases } & \mathbf{3 0}\end{array}$

$\begin{array}{lll}1.6 & \text { Three dimensional structure of transketolases }\end{array}$

$1.7 \quad$ Applications of transketolases in organic synthesis $\quad \mathbf{3 3}$

$\begin{array}{lll}1.8 & \text { Introduction of low barrier hydrogen bonds (LBHBs) } & \mathbf{3 4}\end{array}$

$1.9 \quad$ Role of LBHBs in serine protease $\quad 35$

$\begin{array}{lll}1.10 & \text { LBHBs in phosphate binding proteins } & \mathbf{3 7}\end{array}$

$\begin{array}{lll}1.11 & \text { Aim of the thesis }\end{array}$

2. Materials and Methods 39

$\begin{array}{lll}2.1 & \text { Materials } & 39\end{array}$

2.2 Methods $\quad \mathbf{4 6}$

2.2.1. Molecular biology $\quad \mathbf{4 6}$

2.2.1.1 Concentration determination of DNA 48

2.2.1.2 Site directed mutagenesis using Polymerase Chain Reaction (PCR) 49

2.2.1.3 Agarose gel electrophoresis 449

$\begin{array}{lll}2.2 .1 .4 & \text { DNA sequencing } & 49\end{array}$ 
2.2.1.5 Plasmid transformation $\quad \mathbf{5 0}$

$\begin{array}{ll}2.2 .2 & \mathbf{5 0}\end{array}$

2.2.2.1. Concentration determination of proteins $\quad \mathbf{5 0}$

2.2.2.2. Sodium dodecyl sulfate-polyacrylamide gel electrophoresis (SDS-PAGE) $\quad 50$

$\begin{array}{lll}\text { 2.2.2.3. } & \text { Protein expression } & \mathbf{5 1}\end{array}$

2.2.2.3.1 IPTG induction of protein expression $\quad \mathbf{5 1}$

2.2.2.3.2. Auto-induction of human transketolase $\quad \mathbf{5 1}$

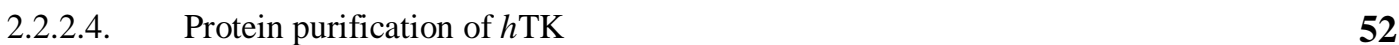

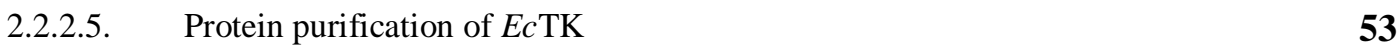

2.2.2.6 Buffer exchange of proteins $\quad \mathbf{5 3}$

2.2.3. Kinetic methods $\quad \mathbf{5 3}$

2.2.3.1. Steady-state activity assay $\quad \mathbf{5 3}$

2.2.3.2 Stopped-flow kinetic methods $\quad \mathbf{5 5}$

$\begin{array}{lll}\text { 2.2.3.2.1 } & \text { Single mixing experiments } & \mathbf{5 5}\end{array}$

2.2.3.2.2 Sequential mixing experiments $\quad \mathbf{5 5}$

2.2.3.3 Temperature jump (T-jump) relaxation technique $\quad \mathbf{5 6}$

2.2.4. Biophysical methods $\quad \mathbf{5 6}$

2.2.4.1. Circular Dichroism (CD) spectroscopy $\quad \mathbf{5 6}$

$\begin{array}{lll}\text { 2.2.4.1.1. } & \text { Active site titration of EcTK } & \mathbf{5 7}\end{array}$

2.2.4.1.2. Analysis of tautomeric forms of ThDP at different $\mathrm{pH}$ values $\quad \mathbf{5 7}$

2.2.4.1.3. $\quad$ Analysis of substrate and ligand binding $\quad \mathbf{5 8}$

2.2.4.2. Analysis of covalent reaction intermediates by acid quench ${ }^{1} \mathrm{H}-\mathrm{NMR} \quad \mathbf{5 8}$ spectroscopy

2.2.4.3. X-ray Crystallography

2.2.4.3.1. Crystallization $\quad 59$

$\begin{array}{lll}\text { 2.2.4.3.2. } & \text { Crystal harvest and substrate soaking } & 60\end{array}$

2.2.4.3.3. Crystal testing in-house $\quad \mathbf{6 1}$ 
2.2.4.3.4. Data collection at the synchrotron $\quad \mathbf{6 1}$

$\begin{array}{lll}\text { 2.2.4.3.5. Data processing } & \mathbf{6 1}\end{array}$

2.2.4.3.6. Model building, refinement and validation $\quad 62$

$\begin{array}{lll}3 . & \text { Results } & 63\end{array}$

3.1. Observation and characterization of a low barrier hydrogen bond (LBHB) 63 in $h \mathrm{TK}$

3.1.1. Capture of a low barrier hydrogen bond (LBHB) in human TK wild type $\quad 63$

3.1.2. Structure of $h$ TK-E160Q reveals the replacement of the LBHB by an 64 ordinary H-bond

3.1.3. Crystal structure evidence of the elongated scissile bond and distorted $\quad \mathbf{6 5}$ covalent intermediate in $h \mathrm{TK}$ E160Q

3.1.4 Crystal structure of $h$ TK-E160Q at resting state $\quad \mathbf{6 8}$

3.1.5 Capture X5P at the docking site in the structure of $h$ TK E366Q variant $\quad 69$

3.1.6. Steady-state kinetic analysis $\quad \mathbf{7 2}$

3.1.7. Pre-steady-state kinetic analysis by stopped-flow spectroscopy $\quad \mathbf{7 4}$

3.1.8 Circular Dichroism (CD) analysis of the tautomeric and protonic states of $\quad \mathbf{7 8}$ ThDP

3.1.9. Temperature jump (T-jump) analysis of the tautomeric and protonic states $\quad \mathbf{8 2}$ of ThDP

3.1.10. Nuclear magnetic renascence (NMR) analysis of intermediates for the donor half reaction

3.2. Capture the post-cleavage intermediate dihydroxyethyl thiamine diphosphate (DHEThDP) with non-phosphorylated substrates xylulose and fructose

3.2.1. Spectroscopic and kinetic analysis of the formation of DHEThDP intermediate by using non- phosphorylated sugars

3.2.2 Structure determination of the DHEThDP intermediate in hTK by using non-phosphorylated sugars

3.2.3. Interactions of the DHEThDP intermediate within the active site of $h \mathrm{TK}$ 
3.2.5. Single-mixing stopped-flow analysis of the donor half reaction using nonphosphorylated sugars

3.2.6. Double-jump stopped-flow analysis for the reactivity of the post-cleavage DHEThDP intermediate from non-phosphorylated sugars with native acceptor R5P

3.2.7. Activation of transketolase by exogenous phosphite dianion for the conversion of non-phosphorylated donor substrates

3.3. Dynamic hotspots in human and E.coli transketolases

3.3.1. Flipping histidine observed in E.coli transketolase

3.3.1.1. Observation of histidine flipping in the crystal structure of EcTK D469N and EcTK D469A

3.3.1.2. Preliminary studies of "histidine flipping"-related active site mutants

3.3.2. Catalytic function study of the intrinsic dynamics in human transketolase

3.3.2.1. Crystal structures of $h \mathrm{TK}$ S427A, Q428A and H77A reveal different active site architectures

3.3.2.2. Preliminary functional studies of the "dynamic hotspots" in human TK

4.1 Function of the low barrier hydrogen bond (LBHB) in $h \mathrm{TK}$

$\begin{array}{lll}\text { 4.1.1 Crystallographic studies on the LBHB } & \mathbf{1 1 8}\end{array}$

$\begin{array}{ll}\text { 4.1.2 } & \mathbf{1 2 1}\end{array}$

4.2 Capture of the central dihydroxyethyl thiamine diphosphate (DHEThDP) intermediate from non-phosphorylated substrates

4.2.1 Accumulation of the DHEThDP intermediate in the crystal structure of human TK with xylulose and fructose

4.2.2 DHEThDP intermediate exists as an enolate form

4.2.3 Kinetical studies on the donor and acceptor half reactions of hTK with nonphosphorylated substrates

4.2.4 Potential application of transketolase using fructose in the field of biocatalysis

4.2.5 Binding energy and enzyme catalysis 
$\begin{array}{ll}\text { 5. Summary and Conclusion } & 136\end{array}$

$\begin{array}{lr}\text { 6. } & 139\end{array}$

$\begin{array}{ll}\text { 7. } & 156\end{array}$

$\begin{array}{lr}\text { Curiculum Vitae } & 172\end{array}$ 


\section{List of Figures}

Fig 1: Structure and nomenclature of thiamine diphosphate.

Fig 2: Activation of cofactor ThDP and the formation of carbene.

Fig 3: Schematic illustration of the protonic and tautomeric states of the aminopyrimidine ring of ThDP.

Fig 4: General reaction mechanism of ThDP-dependent enzymes.

Fig 5: ${ }^{1}$ H-NMR based analysis of the intermediates distribution at equilibrium for the donor half reaction of transketolase with physiological substrates X5P, F6P and artificial substrate HPA.

Fig 6: Pentose phosphate pathway and the major connection points to other metabolic pathways.

Fig 7: General catalytic mechanism of transketolase.

Fig 8: Three dimensional structure of human TK.

Fig 9: Acceptor substrate range for the engineered transketolase.

Fig 10: Energy diagrams for hydrogen bonds as a function of interatomic distance $\left(R_{\mathrm{A}-\mathrm{-}}\right.$ в).

Fig 11: LBHB-facilitated general base mechanism for the acylation of chymotrypsin, the first stage of proteolytic catalysis.

Fig 12: Simplified scheme of the steady-state activity assay of transketolase.

Fig 13: A low barrier hydrogen bond (LBHB) in the crystal structure of human TK wild type in complex with physiological substrate X5P at $0.97 \AA$.

Fig 14: Active site communication in human TK.

Fig 15: An ordinary H-bond is observed in the crystal structure of $h \mathrm{TK}$ E160Q in complex with donor substrate F6P.

Fig 16: Detailed view on the chemical structure of the protonation state for LBHBrelative amino acid residues.

Fig 17: Observation of the elongated scissile bond and physically distorted covalent intermediate in $h$ TK E160Q in complex with F6P.

Fig 18: Active site interactions with the F6P-ThDP intermediate.

Fig 19: Active site architecture of $h \mathrm{TK}$ E160Q at resting state. 
Fig 20: Michaelis complex of $h$ TK-E366Q with donor substrate X5P. $\quad 70$

Fig 21: Active site architecture of $h$ TK E366Q in complex with donor substrate X5P. $\quad 71$

$\begin{array}{ll}\text { Fig 22: } & \text { Steady-state kinetic analysis of } h \text { TK. }\end{array}$

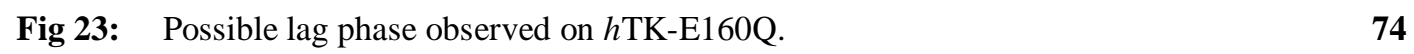

Fig 24: Representative progress curves of the pre-steady-state kinetic analysis of $h$ TK $\quad 75$ and LBHB-related active site variants.

Fig 25: The first phase of the pre-steady-state kinetics for human TK E160Q and E160A. $\quad 76$

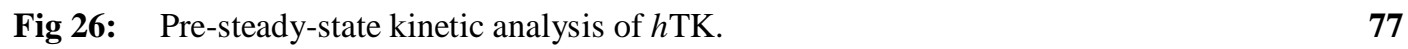

Fig 27: Protonic and tautomeric forms of ThDP and Glu366 in the process of C2-H 79 ionization.

Fig 28: Near-UV CD spectra of the tautomeric and protonic states of ThDP at different $\mathrm{pH}$ values for human TK wild type and LBHB-related active site variants.

Fig 29: Near-UV CD spectra of the tautomeric and protonic states of ThDP at different $\mathrm{pH}$ values for human TK E165Q.

Fig 30: Temperature jump analysis of the tautomeric and protonic forms of ThDP.

Fig 31: ${ }^{1} \mathrm{H}-\mathrm{NMR}$ based analysis of the intermediate distribution at equilibrium after reaction of human TK wild type and LBHB-related active site variants with physiological substrate F6P were acid quenched and isolated.

Fig 32. Two routes for the formation of the covalent DHEThDP intermediate.

Fig 33: ${ }^{1} \mathrm{H}-\mathrm{NMR}$ based analysis of the intermediate distribution at equilibrium after 87 reactions of human TK wild type with physiological substrates F6P/X5P and nonphosphorylated sugars fructose/xylulose were acid quenched and isolated.

Fig 34: UV-Vis difference spectra of $h \mathrm{TK}$ with non-phosphorylated sugars.

Fig 35: Detailed view of the carbanion-enamine intermediate trapped in $h \mathrm{TK}$ by using xylulose at a resolution of $1.5 \AA$.

Fig 36: Hydrogen bonding networks of the DHEThDP intermediate formed by using a.) xylulose and b.) fructose within the active site of $h \mathrm{TK}$.

Fig 37: Binding of post-cleavage product D-glyceraldehyde from xylulose to $h \mathrm{TK}$.

Fig 38: Binding of post-cleavage product D-erythrose from fructose to $h \mathrm{TK}$.

Fig 39: Kinetic analysis for the reaction of the formation of DHEThDP intermediate in $h \mathrm{TK}$ with the non-phosphorylated sugar fructose. 
Fig 40: Comparison of the rate constants for AP formation using fructose and xylulose measured by single-mixing stopped-flow spectroscopy.

Fig 41: Reaction transients for the depletion of DHEThDP intermediate in $h \mathrm{TK}$ with the acceptor substrate R5P.

Fig 42: Kinetic analysis for the reaction of DHEThDP intermediate obtained from nonnative donor substrate fructose with native acceptor substrate R5P.

Fig 43: Kinetic analysis of the effect of phosphite dianion for the formation of DHEThDP intermediate by the non-phosphorylated sugars fructose and xylulose.

Fig 44: Circular Dichroism (CD) measurement for the binding of phosphite and phosphate dianions to the active site of $h \mathrm{TK}$.

Fig 45: Crystal structure of $h \mathrm{TK}$ with phosphite dianion binding at the active site.

Fig 46: Superposition of the crystal structure of $h \mathrm{TK}$ resting state $(1.75 \AA$ PDB accession 106 number: $3 \mathrm{MOS}$ ) with $h \mathrm{TK}$ in complex with phosphite dianion $(1.5 \AA)$.

Fig 47: Detailed view of the "flipping histidine" in the active site of EcTK D469N and D469A mutants.

Fig 48: Near-UV CD spectra of the tautomeric and protonic states of ThDP at different $\mathrm{pH}$ values for EcTK D469N and D469A.

Fig 49: Single turn-over reaction of $E c T K$ D $469 N$ and D469A with donor substrate $\beta$ hydroxypyruvate (HPA).

Fig 50: Reconstitution of holo-EcTK from cofactor ThDP binding to apoenzyme.

Fig 51: Detailed view of the "dynamic hotspots" in human TK and related active site mutations.

Fig 52: Stopped-flow analysis of the pre-steady-state kinetic of the $h \mathrm{TK}$ donor half reaction.

Fig 53: Near-UV CD spectra of the tautomeric and protonic states of ThDP at different $\mathrm{pH}$ values for $h \mathrm{TK}$ “dynamic hotspots”-related active site variants.

Fig 54: Sequence alignment of transketolases from different species.

Fig 55: Minimal reaction sequence for the pre-steady-state reaction of transketolase (donor half reaction).

Fig 56: Superposition of the ThDP molecule in holo-hTK (red) and covalent reaction intermediates DHEThDP in hTK soaked with xylulose (left panel, yellow) and fructose (right panel, blue).

Fig 57: Mechanism for the formation of enolate form of DHEThDP. 
Fig 58: Conformational equilibrium compositions of sugars in aqueous solution.

Fig 59: A possible low barrier hydrogen bond (LBHB) observed in the crystal structure of EcTK-D469N at a resolution of $1.06 \AA$.

Fig 60: Temperature jump analysis of human TK E160Q.

Fig 61: Steady-state kinetic analysis of hunan TK wild type and LBHB-related active site variants.

Fig 62: Detailed view of the carbanion-enamine intermediate trapped in hTK by using fructose at a resolution of $1.5 \AA$.

Fig 63: Photodiode array based stopped-flow analysis of human transketolase with nonphosphorylated sugar fructose.

Fig 64: Single-mixing stopped-flow analysis of human TK with fructose.

Fig 65: Single-mixing stopped-flow analysis of E.coli TK with fructose.

Fig 66: Circular Dichroism (CD) spectra of substrate binding to human and E.coli TK.

Fig 67: "Flipping histidine" observed in the active site of EcTK D469N apo-enzyme.

Fig 68: Single-mixing stopped-flow analysis of EcTK-D469N and EcTK-D469A with physiological substrate fructose 6-phosphate (F6P).

Fig 69: Purification of hTK for crystallization. 


\section{List of Tables}

Table 1: $\quad$ List of utilized mutagenesis and sequencing primers of $h \mathrm{TK}$ and $E c \mathrm{TK}$

Table 2: $\quad$ Substrates and conditions for ligand binding CD measurement.

Table 3: Conditions for substrate soaking experiments and data collection

Table 4: Overview of the steady-state kinetic constants for $h$ TK wild type and LBHBrelated active site variants.

Table 5: Overview of the pre-steady-state kinetic constants for $h \mathrm{TK}$ wild type and LBHB-related active site variants.

Table 6: X-ray statistics for the crystal structures of human transketolase E160Q ground state, E160Q in covalent complex with donor substrate fructose 6phosphate (F6P) and E366Q in non-covalent complex with donor substrate xylulose 5-phosphate (X5P).

Table 7: X-ray statistics for the crystal structures of human transketolase wild type in complex with non-phosphorylated sugars xylulose and fructose as well as human transketolase wild type with activating phosphite dianion.

Table 8: $\quad$ X-ray statistics for the crystal structures of human transketolase active site variants Q428A, S427A and H77A in complex with donor substrate fructose 6-phosphate (F6P).

Table 9: X-ray statistics for the crystal structures of E.coli transketolase active site variants D469N, D469A holo-enzyme in ground state and D469N apoenzyme.

Table 10: Analysis of the hydrogen bond distance at the same LBHB position in other transketolase structures. 


\section{Abbreviations}

AcThDP

Ala

AHAS

Amp

AP

$\mathbf{A P H}^{+}$

app

Apo

Arg

Asn

Asp

BSA

CD

CoA

Da

DHEThDP

DFT

DHAP

DXS

DXP

E4P

E. coli

EcTK

EDO

EDTA

F6P
Acetyl-ThDP

Alanine

Acetolactate synthase

Ampicillin

Aminopyrimidine

Aminopyrimidinium

Apparent

Apo-enzyme, enzyme without cofactor

Arginine

Asparagine

Asparaginic acid

Bovine serum albumin

Circular dichroism

Coenzyme A

Dalton

Dihydroxyethyl thiamine diphosphate

Density functional theory

Dihydroxyacetone phosphate

1-Deoxy-d-xylulose 5-phosphate synthase

1-Deoxy-d-xylulose 5-phosphate

D-erythrose 4-phosphate

Escherichia coli

Transketolase from escherichia coli

1,2-ethanediol

Ethylendiamintetraacetate

D-fructose 6-phosphate 


\begin{tabular}{|c|c|}
\hline GAP & D-glyceraldehyde 3-phosphate \\
\hline G3P-DH & Glycerol 3-phosphate dehydrogenase \\
\hline GA & Glycol aldehyde \\
\hline Gln & Glutamine \\
\hline Glu & Glutamic acid \\
\hline Gly & Glycine \\
\hline His & Histidine \\
\hline His-tag & Hexa-histidine tag \\
\hline HL-ThDP & Hydroxylactyl thiamin diphosphate \\
\hline HPA & $\beta$-hydroxypyruvate \\
\hline$h \mathbf{T K}$ & Human transketolase \\
\hline Ile & Isoleucine \\
\hline IP & Iminopyrimidine \\
\hline IPTG & Isopropyl- $\beta$-D-thiogalactoside \\
\hline $\boldsymbol{k}$ & Rate constant \\
\hline$K_{\mathbf{D}}$ & Dissociation constant \\
\hline $\boldsymbol{K}$ & Equilibrium constant \\
\hline LB & Luria-bertani-media \\
\hline LBHB & Low-barrier hydrogen bond \\
\hline Leu & Leucine \\
\hline Lys & Lysine \\
\hline MES & 2-(N-morpholino) ethanesulfonic acid \\
\hline Met & Methionine \\
\hline MEP & Methylerythritol 4-phosphate \\
\hline MW & Molecular weight \\
\hline MWCO & Molecular weight cut-off \\
\hline NADH/NAD ${ }^{+}$ & Nicotinamide adenine dinucleotide (reduced form/oxidized form) \\
\hline nd & Not determined \\
\hline $\mathrm{Ni}^{2+}-\mathbf{N T A}$ & $\mathrm{Ni}^{2+}-\mathrm{Nitrilotriacetic}$ acid \\
\hline
\end{tabular}




\begin{tabular}{|c|c|}
\hline NMR & Nuclear magnetic resonance \\
\hline obs & Observed \\
\hline OD & Optical density \\
\hline OMPDC & Orotidine 5'-monophosphate decarboxylase \\
\hline PAGE & Polyacrylamide gel electrophoresis \\
\hline PCR & Polymerase chain reaction \\
\hline PDB & Protein data bank \\
\hline PDC & Pyruvate decarboxylase \\
\hline PDH & Pyruvate dehydrogenase \\
\hline PEG & Polyethylene glycol \\
\hline pH & Negative logarithm of $\mathrm{H}^{+}$concentration \\
\hline Phe & Phenylalanine \\
\hline PMSF & Phenylmethylsulfonylfluorid \\
\hline POX & Pyruvate oxidase \\
\hline PPP & Pentose phosphate pathway \\
\hline Pro & Proline \\
\hline QM/MM & Quantum Mechanical/Molecular Mechanical \\
\hline R5P & D-ribose 5-phosphate \\
\hline rmsd & Root-mean-square deviation \\
\hline rpm & Revolutions per minute \\
\hline RT & Room temperature \\
\hline S7P & D-sedoheptulose 7-phosphate \\
\hline SDS & Sodium dodecylsulfate \\
\hline SEC & Size exclusion chromatography \\
\hline Ser & Serine \\
\hline SLS & Swiss light source \\
\hline TAL & Transaldolase \\
\hline TCA & Trichloro acetic acid \\
\hline TEMED & Tetramethylethylenediamine \\
\hline
\end{tabular}


ThDP Thiamin diphosphate

Thr Threonine

TIM Triose-phosphate isomerase

Thr Threonine

TK Transketolase

Tris Tris(hydroxymethyl)aminomethane

Trp Tryptophane

Tyr Tyrosine

UV/Vis Ultraviolet and visible fraction of electromagnetic radiation

Val Valine

wt Wild type

X5P D-xylulose 5-phosphate 


\section{Acknowledgements}

I would like to thank Prof. Dr. Kai Tittmann for the supervision of this PhD thesis and his continuous support during the last four years. I would also like to thank him for his numerous useful ideas, discussions and encouragements. Furthermore, I would like to thank him for the NMR data collection and for teaching me fast kinetic techniques.

I would like to thank Prof. Dr. Ralf Ficner for external co-supervision of this thesis and for taking part in the thesis committee. In addition, I would like to thank him for his generous ability to use the instruments and facilities of his department. I would like to thank Dr. Till Ischebeck for being a member of my thesis committee.

I am also thankful to Prof. Dr. Ivo Feussner, Prof. Dr. Jörg Stülke, Prof. Dr. Ricardo Mata, and Dr. Manfred Konrad for kindly accepting to be the members of the examination board, despite their busy schedules.

I am deeply thankful to Dr. Piotr Neumann for his continuous support in all the theoretical and practical aspects of protein crystallography. Especially, I would like to thank him for carrying out the majority of the X-ray data processing work as well as helping with harvest very fragile crystals. I would like to thank him for his suggestions and comments on the structure refinement.

I would like to thank all the former and present members of the department of Molecular Enzymology (the old name is department of Bioanalytics) for their help in the daily lab work and the great working atmosphere. First, I would like to thank Dr. Danilo Meyer for helping me in the initial phase of my $\mathrm{PhD}$ and for introducing me all the basic things in the lab. I would like to thank Dr. Kathrin Schröder-Tittmann for her support in the protein purification. Thanks to Dr. Florian Brodhun for organizing all the basic stuffs in the daily lab work. I would like to thank Dr. Victor Sautner for his continuous support in the protein crystallographic data analysis and the discussion on kinetics and thermodynamics problems. Thanks to Fabian Nikolaus Rabe von Pappenheim and Lisa-Marie Funk for the discussion of the LBHB project. I would like to thank Fabian Nikolaus Rabe von Pappenheim, Fabienne Libuda, Sabin Prajapati, Sören Rindfleisch and Dr. Oliver Kupski for proofreading of the thesis.

I would like to thank Dr. Oliver Kupski for collecting X-ray data for a collaborative project with Prof. Dr. Paul Dalby at the University College London.

Finally, I am grateful to my parents for their endless support during my studies and Dan $\mathrm{Yu}$ for her unconditional support. 


\section{Introduction}

\subsection{Thiamine and the biological function of its active forms}

Thiamine (Vitamin B1) serves as an indispensable component in all prokaryotes and eukaryotes with both enzymatic and non-enzymatic functions (Bettendorff and Wins, 2009). It plays pivotal roles in numerous primary metabolic pathways such as the synthesis of carbohydrates and branched-chain amino acids (Bunik, 2013). Thiamine also involves in the production of neurotransmitters and nucleic acids by the courtesy of Pentose Phosphate Pathway (PPP) which produces ribose 5-phosphate, a precursor compound for the synthesis of nucleic acids (Bunik et al., 2013). As an essential micronutrient for human beings, the intake of thiamine exclusively relies on dietary supplies as it can not be endogenously synthesized by the human body (Bettendorff, 1994; Bettendorff and Wins, 2009).

Thiamine deficiency would lead to a variety of diseases and some even have life-threatening consequences. One of the most well-known disease is named beriberi, which has been documented since ancient China (approx. 2600 BC), is a cluster of symptoms including loss of weight, impaired heart function, emotional disturbance and weakened limb (Zahr et al., 2011). The reason for causing beriberi in Asian countries was not clear until the end of the 19th century. In regions where the polished rice was served as the main staple food, rice husk which is the primary supply of thiamine was removed, causing thiamine deficiency in the population (Goselink et al., 2012). Later on, patients with beriberi were treated with a thiamine-rich diet such as whole grain brown bread and they were gradually recovered. Beriberi has been categorised into four groups and one of them is named "wet beriberi" which specially causes damages to the cardiovascular system and is sometimes fatal due to the cause of heart failure (Stroh et al., 2014).

In vivo, thiamine can be phosphorylated at the thiazole hydroxyethyl group, which leads to the formation of thiamine mono-, di- and tri-phosphates (Rindi, 1982). Thiamine triphosphates and the adenosine conjugates were shown as signal molecules in prokaryotes when they are under carbon or amino acid starvation conditions (Bettendorff et al., 2007). The most important thiamine derivative is the di-phosphate ester thiamine diphosphate or thiamine pyrophosphate (ThDP), which is the biological active form of thiamine. ThDP acts as a crucial cofactor for many metabolic enzymes such as pyruvate dehydrogenase (PDH) multi-enzyme complex which links glycolysis to the citric acid cycle and transketolases in the Pentose Phosphate Pathway (PPP). These enzymes catalyse a broad range of reactions (Kluger and Tittmann, 2008). Despite of the universal of ThDP dependent enzymes, the catalytic 
mechanisms share a common feature that similar cofactor-derived intermediates are involved in the reaction (Schellenberger, 1998).

\subsection{Reactivity of ThDP}

The chemical structure of thiamine was proposed by Williams and colleagues in 1935 and 1936 (Williams, 1935, 1936), and later on Williams and Waterman established a synthetic strategy to chemically synthesize thiamine, proved the chemical structure and also provided a practical and commercially available route for the large scale preparation of thiamine (Williams and Cline, 1936). Since then, the mechanistic study of thiamine had dramatically increased and people had proposed a variety of reaction mechanisms until Breslow identified the correct reactive center of ThDP (Breslow, 1957). As shown in Fig 1, the cofactor ThDP is composed of two ring systems, the six-membered aminopyrimidine and the five-membered thiazolium, and they are connected by a methylene bridge. A diphosphate anchor is connected to the other side of the thiazolium ring and is proposed to facilitate the binding of cofactor to the active sites of ThDP-dependent enzymes (Kluger and Tittmann, 2008). Breslow first proposed the methylene group to be the ionization site to form the nucleophilic carbanion, but this was rejected by Westheimer (Fry et al., 1957) by showing that neither thiamine nor ThDP could incorporate deuterium at the methylene bridge site. After this failure, Breslow examined the deuterium exchange at the adjacent $\mathrm{C}-\mathrm{H}$ on the thiazolium ring and finally discovered the activation mechanism of ThDP (Breslow, 1957). He proposed that the formation of the C2carbanion is the first step in the ThDP-dependent enzyme reactions, which gained well acceptance and was confirmed in many enzymes that use ThDP as cofactor (Kluger, 1990; Kluger and Tittmann, 2008).

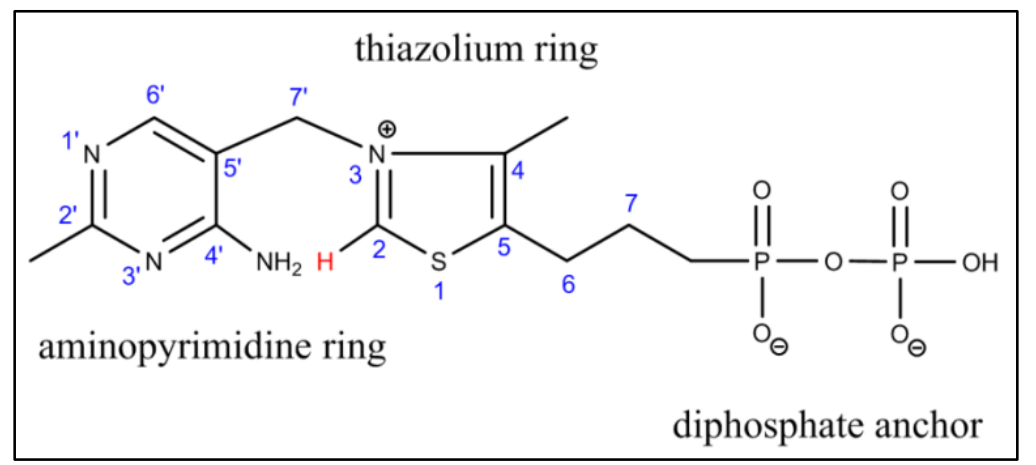

Fig 1: Structure and nomenclature of thiamine diphosphate. Chemical structure of ThDP is composed of a six-membered aminopyrimidine ring, a five-membered thiazolium ring and a diphosphate (or pyrophosphate) moiety. The diphosphate moiety is also named "anchor" because of the function for facilitating the binding of ThDP to the active sites of ThDP-dependent enzymes. The acidic hydrogen at the $\mathrm{C} 2$ position is shown in red. 


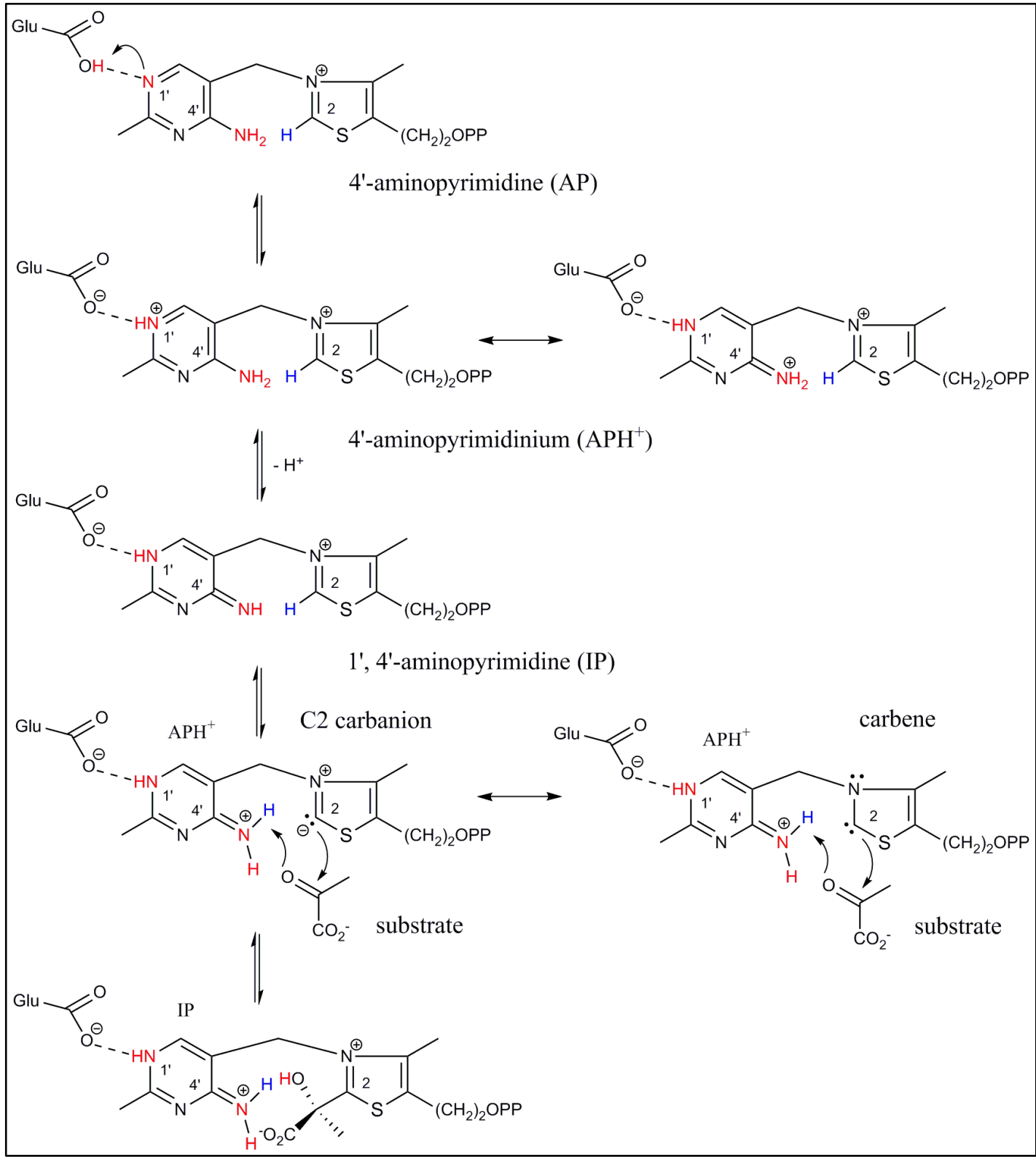

Fig 2: Activation of the cofactor ThDP and the formation of carbene. The figure was prepared according to Kluger and Tittmann (2008) and Meyer et.al. (2013) with minor modifications. The protonic and tautomeric states of the six-membered aminopyrimidine ring ( $\mathrm{AP}, \mathrm{IP}$ and $\mathrm{APH}^{+}$) are indicated. The conserved glutamate interacting with the N1' of thiamine is shown with hydrogen bonding in black dashed line. The accumulation of carbene resonance form is highlighted as well.

Subsequent studies revealed that $\mathrm{ThDP}$ on its own only shows very low catalytic activity under physiological conditions. The reason for this behaviour is mainly that the $\mathrm{C} 2$ of thiazolium has a $\mathrm{pKa}$ around 17-19 and exhibits low acidity under physiological $\mathrm{pH}$ (Washabaugh and Jencks, 1988). As a consequence of that, only small amount of the reactive nucleophilic form could be generated, leading to the low activity of free ThDP. However, when the cofactor binds to 
the active site of a ThDP-dependent enzyme, the $\mathrm{C} 2$ deprotonation velocity was observed to be dramatically increased when PDC and transketolase were studied (Hübner et al., 1998; Kern et al., 1997). This result had also excluded the proposal in an early model that the ThDP C2carbanion is stabilised in the enzyme-bound state (Crosby and Lienhard, 1970; Kern et al., 1997). Moreover, Breslow had proposed that carbenes (Breslow, 1957), which are highly reactive chemical compounds, may occur transiently in the catalytic cycle of ThDP dependent enzymes. But the existence of these short-lived compounds was not confirmed until Meyer and colleagues (Meyer et al., 2013; Neumann and Tittmann, 2014) observed the accumulation of a stable carbene as the major resonance form of thiamine in the crystal structure of pyruvate oxidase in complex with a substrate mimic.

The covalent reaction of ThDP-dependent enzymes only happens at the $\mathrm{C} 2$ position of the five-membered thiazolium ring, which is similar at the initial step but will diversify in the subsequent steps among ThDP-dependent enzymes (Frank et al., 2007a; Kluger and Tittmann, 2008; Schellenberger, 1998). The six-membered aminopyrimidine ring was later postulated to have co-catalytic function, especially at the cofactor activation step (Schellenberger, 1998). Earlier work done by Hübner and colleagues have clearly revealed the catalytic importance of the N1' and N4' nitrogen atoms of the aminopyrimidine ring by employing functional, kinetic analysis of cofactor analogues, site-directed mutagenesis and NMR-based proton/deuterium exchange methods (Kern et al., 1997). Pioneering crystallographic work of several ThDPdependent enzymes such as TK, POX and PDC have pointed out a V-conformation of ThDP after incorporation into the active sites. This conformation brings the $\mathrm{C} 2$ of thiazolium and the 4'-amino group of aminopyrimidine in a very close proximity (Muller et al., 1993). Based on those structural and functional results, a currently well-accepted mechanism for the cofactor activation was proposed which involves both ring systems of ThDP. As shown in Fig 2, a glutamate residue hydrogen bonding with the N1' atom first donates its proton to the 4'aminopyrimidine (AP), leading to the formation of the conjugate acid form 4'aminopyrimidinium $\left(\mathrm{APH}^{+}\right)$. The $\mathrm{APH}^{+}$form then losses one proton to generate the 1',4'iminopyrimidine (IP) form, a tautomeric form of the AP, whose 4'-imino function acts as a general base to deprotonate the C2 on the thiazolium (Jordan, 2003; Nemeria et al., 2004, 2009). This proton relay assisted by the "catalytic triad" of glutamate-iminopyrimidine-thiazol has been assumed to be a paradigm for the cofactor activation. But in 2008, the crystal structure of one ThDP-dependent enzyme named glyoxylate carboligase revealed the substitution of the conserved glutamate by a valine (Kaplun et al., 2008; Shaanan and Chipman, 2009). This unexpected finding thus brought a modification of the activation mechanism. Recently, computational Quantum Mechanical/Molecular Mechanical (QM/MM) methods performed on transketolase (TK) (Nauton et al., 2016) and 1-Deoxy-D-xylulose 5-phosphate synthase (DXS) 
(White et al., 2016) have revealed a new route for ThDP activation which involves a histidine near the N4' atom and a water molecule. These subversive hypotheses gained from computational calculation therefore need to be tested experimentally.

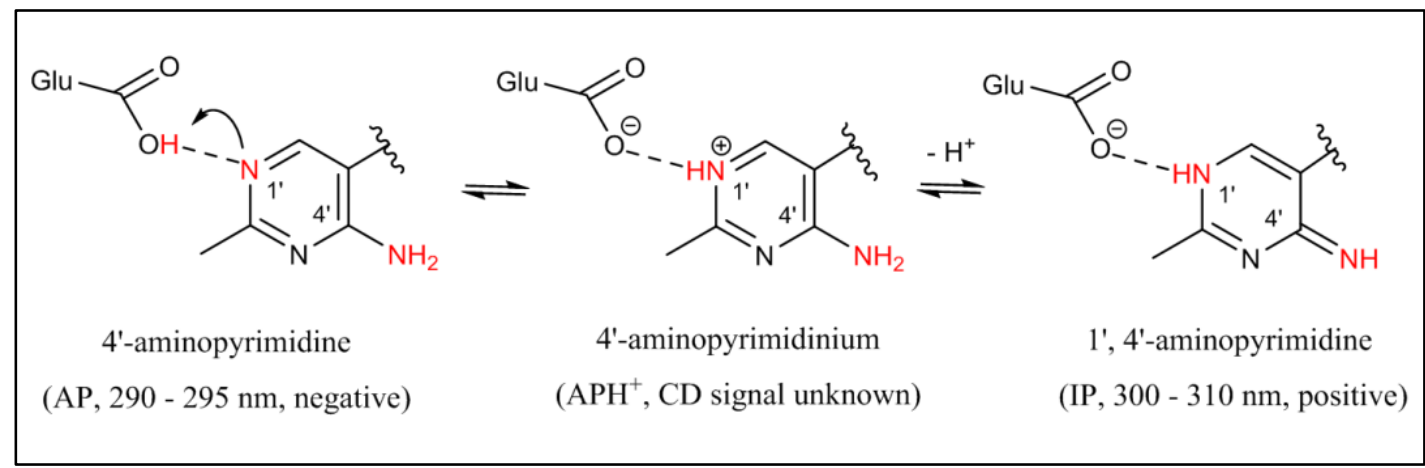

Fig 3: Schematic illustration of the protonic and tautomeric states of the aminopyrimidine ring of

ThDP. The transfer of hydrogen atoms between the three tautomeric forms (AP, IP and $\mathrm{APH}^{+}$) are highlighted in red and the corresponding CD signatures are indicated.

In addition to the activation of the cofactor, the aminopyrimidine ring is proposed to function in multiple subsequent steps in ThDP dependent enzymes (Nemeria et al., 2009). The three protonic and tautomeric forms are supposed to play individual roles either as acid or base in different reaction steps. The $\mathrm{pKa}$ values for the $([\mathrm{AP}]+[\mathrm{IP}]) /\left[\mathrm{APH}^{+}\right]$equilibrium were determined for several ThDP dependent enzymes. The coincidence of the $\mathrm{pKa}$ with the optimum $\mathrm{pH}$ for enzymatic activity indicates that all three forms (AP, IP and $\mathrm{APH}^{+}$) must coexist during the reaction path (Nemeria et al., 2004). In order to characterize those protonic and tautomeric states on ThDP dependent enzymes, the group of Jordan spent a considerable time to synthesize model compounds and assigned the AP and IP form by UV-Vis and Circular Dichroism (CD) spectroscopy (Jordan et al., 2002; Nemeria et al., 2004, 2007). According to their findings, the AP tautomer exhibits a negative $\mathrm{CD}$ signal and a corresponding absorbance band at 320-330 nm, while the IP form gives rise to a positive CD signal and absorbs at 300$310 \mathrm{~nm}$ (Lüdtke et al., 2013). The spectra signature of the conjugate acid $\mathrm{APH}^{+}$form so far could not be unambiguously assigned, but recent studies on PDC and phosphoketolase have revealed a negative $\mathrm{CD}$ band at $290 \mathrm{~nm}$ which is very likely to be the $\mathrm{APH}^{+}$form (Schneider, 2014; Wechsler, 2014). 


\subsection{Covalent intermediates in ThDP catalysis}

After losing the $\mathrm{C} 2$ proton on the thiazolium of ThDP, the resulting carbanion functions as a nucleophile which attacks the carbonyl group of a variety of substrates including 2-keto acid (in particular pyruvate), phosphate derivatives of monosaccharides and other more complicated donor substrates (Kluger and Tittmann, 2008). The addition changes the electronic properties of the cofactor and the substrates are hold in a precise position, which is the common background for numerous biochemical reactions using ThDP. These reactions share a common feature that involves the so-called "Breslow intermediate" which is the carbanion/enamine form generated by the cleavage of the initial covalent substrate-ThDP adduct (Bunik, 2013).

Although ThDP-dependent enzymes catalyze a wild variety of reactions in different metabolic pathways, those reactions share a common mechanism that involves analogous covalent intermediates (Fig 4). In recent years, the direct observation of many of those intermediates has become possible due to the development of a variety of chemical and biophysical methods (Kluger and Tittmann, 2008; Tittmann et al., 2003; Wille et al., 2006). More and more proposed intermediates that were only theoretically postulated in the past, have now been observed. As shown in Fig 4, the first covalent intermediate in the reaction sequence of all ThDP-dependent enzymes is the sp3-hybridized substrate-ThDP covalent adduct which is formed after the nucleophilic attack of the $\mathrm{C} 2$-carbanion on the carbonyl group of substrates. This intermediate or analogues have been observed by $\mathrm{x}$-ray crystallography in many ThDPdependent enzymes such as transketolase (TK) (Asztalos et al., 2007; Lüdtke et al., 2013), pyruvate decarboxylase (PDC) (Kutter et al., 2009; Meyer et al., 2010), benzoylformate decarboxylase (BFD) (Bruning et al., 2009) and pyruvate oxidase (POX) (Meyer et al., 2012; Tittmann and Wille, 2009; Wille et al., 2006). These structures have delineated an identical mechanistic features for the intermediate alignment, especially for the enzymes that take pyruvate as the first substrate (Kluger and Tittmann, 2008). The common features for this intermediate are summarized as follows: 1.) the scissile substrate bond is found to be at the perpendicular position relative to the five-membered thiazolium ring (Wille et al., 2006); 2.) A term named maximum overlap mechanism, that is the positively charged thiazolium ring serves as an optimal electron sink for the electron pair which is formed after the bond cleavage (Turano et al., 1982), could also be applied to ThDP enzymes (Asztalos et al., 2007; Meyer et al., 2010; Wille et al., 2006). An unexpected but reasonable structural observation was that the $\mathrm{C}-\mathrm{C}$ single bond connecting substrates and the thiazolium ring exhibits a strong deviation from planarity of up to $30^{\circ}$ (Asztalos et al., 2007; Lüdtke et al., 2013). In addition, the scissile C2$\mathrm{C} 3$ bond is elongated to around $1.6 \AA$ which is approx. $0.1 \AA$ longer than the normal C-C single 
bonds (Lüdtke et al., 2013). These high-energy states of intermediates are thought to have the ability to reduce the barrier for the subsequent steps, thus enhancing the overall efficiency for the enzymatic catalysis (Lüdtke et al., 2013; Neumann and Tittmann, 2014; Tittmann, 2014).

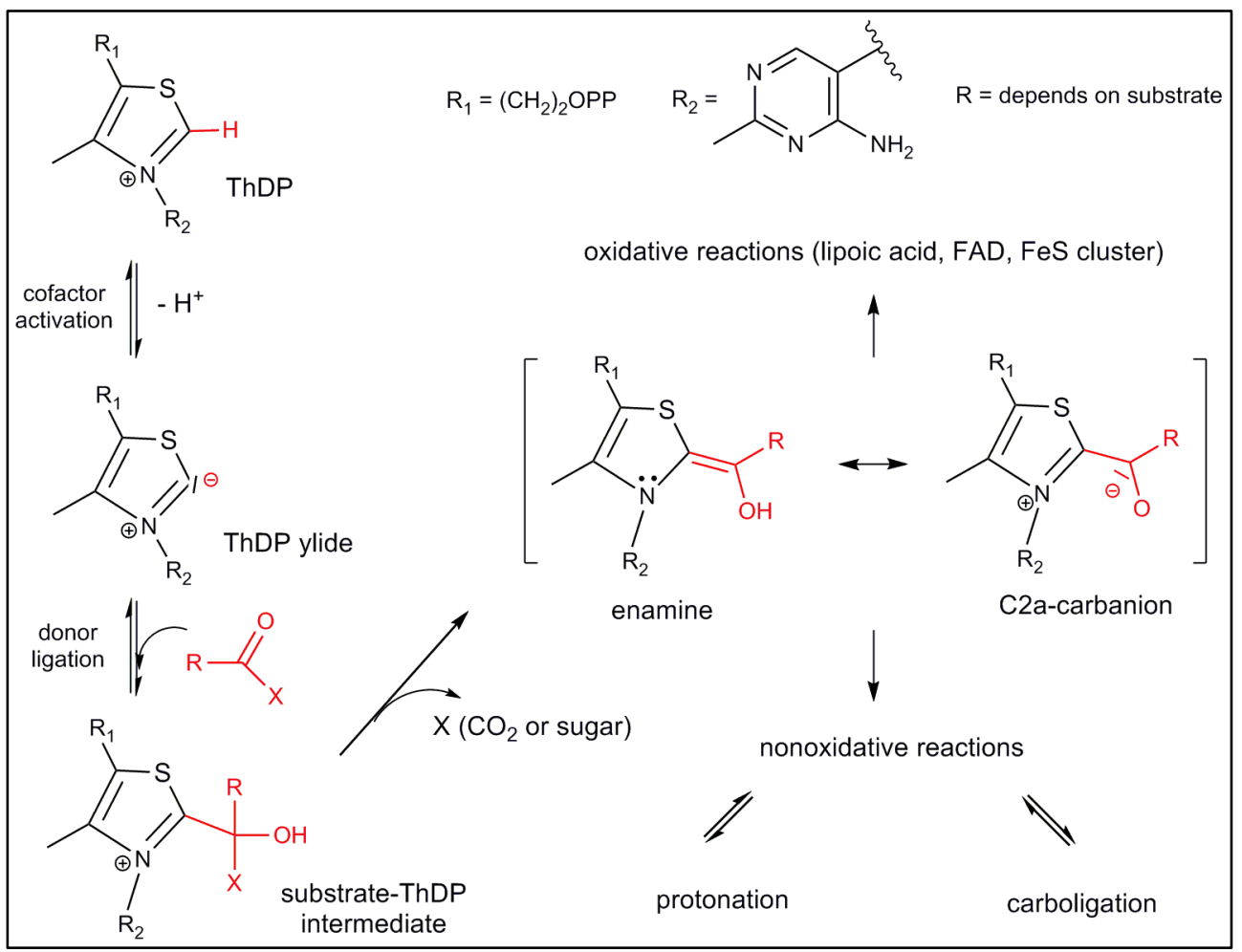

Fig 4: General reaction mechanism of ThDP-dependent enzymes. The analogous intermediates are highlighted in red and different fates of those intermediates are indicated.

After the cleavage of the substrate-ThDP intermediate, the reaction trajectory reaches a central branching point, the formation of the post-cleavage $\mathrm{C} 2 \alpha$-carbanion/enamine intermediate. The chemical features of this intermediate have been characterized as the sp3 hybridized $\mathrm{C} 2 \alpha$ carbanion in resonance with the sp2 hybridized enamine form (Kluger and Tittmann, 2008). In the following reaction steps, this intermediate may proceed through either non-oxidative or oxidative reactions. Two possible reactions can generally happen in the non-oxidative direction, protonation at the $\mathrm{C} 2 \alpha$ to generate HEThDP intermediate with the elimination of acetaldehyde in ThDP-dependent decarboxylases (Meyer et al., 2010) and carboligation of another keto-acid or sugar phosphate to the formation of a conjugate in acetohydroxy acid synthases (AHAS) (Belenky et al., 2012) or transketolases (TK) (Asztalos et al., 2007). In the oxidative pathway, the carbanion/enamine intermediate will be oxidized by neighbouring redox-active cofactors such as lipoamide (PDHc) (Ciszak et al., 2003), flavins (POX) 
(Tittmann et al., 2002) or $\mathrm{Fe}_{4}-\mathrm{S}_{4}$ clusters (PFOR) (Chabrière et al., 2001). The generated acetyl-ThDP (AcThDP) intermediate can further react to produce 8-acetyl-dihydrolipoamide (PDHc), acetyl-CoA (PFOR), acetate and acetylphosphate (POX).

Spectroscopic observation of this intermediate was first reported by Jordan and colleagues that an enamine analogue binding to PDC exhibits a UV-Vis absorbance at $440 \mathrm{~nm}$ (Kuo and Jordan, 1983). Subsequent studies with substrate analogues of BFD (Sergienko et al., 2000) and IPDC (Schütz et al., 2005a) revealed the $\lambda_{\max }$ for the enamine is 400 and $380 \mathrm{~nm}$, respectively. Recently, the formation and depletion of the enamine analogue DHEThDP intermediate was measured kinetically at $300 \mathrm{~nm}$ by a rapid-mixing technique (Asztalos et al., 2007; Golbik et al., 2005). In addition, the structural snapshots of this central intermediate have been observed in several ThDP-dependent enzymes such as ScTK (Fiedler et al., 2002), POX (Meyer et al., 2012; Wille et al., 2006), phosphoketolase (Suzuki et al., 2010), BCKDC (Machius et al., 2006) and oxalyl-coenzyme A (CoA) decarboxylase (Berthold et al., 2007). Some of those results suggest a planar, enamine-like state, while others lead to different conclusions like the accumulation of the carbanion state or the non-planar state of enamine. Furthermore, a recently released crystal structure of pyruvate oxidase with super-resolution revealed that the intermediate samples between the carbanion and a newly proposed ketone in a tautomeric equilibrium (Meyer et al., 2012). Although a lot of information could be obtained from these structures, there are still some questions that need to be addressed. For example, several researches have challenged the existence of the resonance-stabilized enamine form which is believed to be a paradigm in the ThDP dependent enzymes reactions, so a question arises if the enamine intermediate is actually necessary in thiamine catalysis (Amara et al., 2008; Berkessel et al., 2010). Another question would be that how enzymes can stabilize the $\mathrm{C} 2 \alpha$-carbanion which is very reactive and prone to get protonated.

In addition to X-ray crystallography, a NMR-based chemical quench method has been developed recently which enables the identification and quantification of the covalent intermediates during ThDP catalysis (Tittmann et al., 2003). Chemically or chemoenzymatically synthesized intermediates were used as standards and can be distinguished unambiguously by the ${ }^{1} \mathrm{H}-\mathrm{NMR}$ chemical shifts of the C6'-H singlets. This method has been successfully applied to a variety of ThDP-dependent enzymes and the quantitative analysis of intermediates by defined time points made the determination of microscopic rate constants of individual steps possible for multi-step reaction mechanisms (Kluger and Tittmann, 2008). In addition, the combination of this method with site-directed mutagenesis enables a more comprehensive study of individual active site residues (Tittmann et al., 2003). The covalent intermediates in the donor half reaction of transketolase have also been extensively studied by 
this approach (Fig 5). Chemical shifts of the C6'-H singlets of intermediates X5P-ThDP, F6PThDP and DHEThDP are 7.35, 7.34 and $7.31 \mathrm{ppm}$ respectively, which allows an unambiguous assignment of each intermediate during catalysis (Asztalos et al., 2007; Mitschke et al., 2010). Under equilibrium conditions, covalent adduct X5P-ThDP and F6P-ThDP are stabilized at the active site of transketolase while the post-cleavage intermediate DHEThDP solely accumulates when an artificial substrate $\beta$-hydroxypyruvate (HPA) is used as donor (Lüdtke, 2012). The release of $\mathrm{CO}_{2}$ renders this reaction irreversible, which makes HPA an ideal donor substrate in the bio-synthetic reactions using transketolases as catalysts (Hailes et al., 2013).

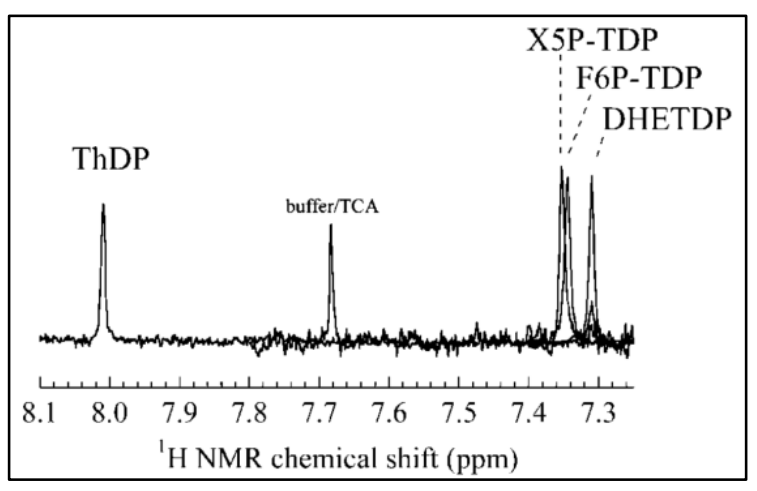

Fig 5: ${ }^{1} \mathrm{H}-\mathrm{NMR}$ based analysis of the intermediates distribution for the donor half reaction of transketolase with physiological substrates X5P, F6P and artificial substrate HPA. C6'-H fingerprints of X5P-ThDP, F6PThDP, DHEThDP intermediates and free ThDP are indicated. Figure is adapted from (Kluger and Tittmann, 2008).

\subsection{Biological functions of ThDP-dependent enzymes and transketolases}

ThDP-dependent enzymes represent a huge family of enzymes which play essential roles in a variety of anabolic and catabolic pathways, such as the Krebs cycle (also known as the tricarboxylic acid cycle) (Frank et al., 2007b), non-mevalonate isoprenoid biosynthesis in plants and bacteria (Lichtenthaler, 1999) and the non-oxidative branch of pentose phosphate pathway (Lindqvist et al., 1992). Several non-mammalian enzymes have been identified as targets for fungicides, herbicides or antimicrobial compounds (Bunik et al., 2013). One of those examples is acetohydroxyacid synthase (AHAS) which is absent in mammals but is responsible for the biosynthesis of the branched chain amino acids in plants, fungi and bacteria (Chipman et al., 2005; LaRossa and Schloss, 1984). Several compounds have been discovered to serve as herbicides by inhibiting the activity of AHAS (Gedi and Yoon, 2012). Another antibacterial target of ThDP-dependent enzymes is 1-deoxy-D-xylulose 5-phosphate synthase (DXS) which is the first committed enzyme in the methylerythritol 4-phosphate (MEP) pathway (also named non-mevalonate pathway) for isoprenoids biosynthesis (Du et al., 2011; Lichtenthaler, 2000). The fact that this pathway only exists in plants and microorganisms but 
absent in human makes DXS an ideal target for antibacterial agents or herbicides (Rohdich et al., 2001). Compounds like clomazone and 5-ketoclomazone have been proven to inhibit DXS and the inhibition mechanisms are revealed as well (Matsue et al., 2010; Mueller et al., 2000). Transketolases (EC 2.2.1.1) could be found in almost all tissues in animals and plants (Kochetov, 1982) and contain ThDP as a prosthetic group and require bivalent cations for the catalytic activity (Schenk et al., 1998; Schneider and Lindqvist, 1998; Sprenger et al., 1995). Transketolases are mainly involved in two metabolic pathways, namely the Calvin cycle of photosynthesis and the Pentose-Phosphate Pathway (PPP) (Soderberg, 2005). In the first reaction of the non-oxidative branch of PPP, TKs catalyse the transfer of a C2-carbon fragment from a C5-ketose (D-xylulose 5-phosphate, X5P) to a C5-aldose (D-ribose 5-phosphate, R5P) to form a C7-ketose (sedoheptulose 7-phosphate, S7P). One molecule of glyceraldehyde 3phosphate (G3P) is obtained thereof due to the release of a C2-carbon fragment. In the Calvin cycle, the reverse reaction of the conversion from S7P and G3P to X5P and R5P is catalysed by TKs (Raines, 2003). The second reaction catalysed by TKs in PPP pathway is the transfer of a 2-carbon fragment from the C5-ketose (D-xylulose 5-phosphate, $\mathrm{X} 5 \mathrm{P}$ ) to a C4-aldose (Derythrose 4-phosphate, E4P) to give a C6-ketose (D-Fructose 6-phosphate, F6P) and a C3aldose (D-Glyceraldehyde 3-phosphate, G3P) as products. Again, in the Calvin cycle the reverse reaction occurs (Raines, 2003; Soderberg, 2005).

As shown in Fig 6, the Pentose Phosphate Pathway generally consists of two distinct phases. The oxidative phase generates two molecules of NADPH which serves as the reducing agent to sustain the glutathione level via glutathione reductase (Kruger and von Schaewen, 2003). This function is very important in erythrocytes whose main responsibility is to transfer oxygen to different tissues. A major disadvantage caused by this process is the accumulation of the high concentration of reactive oxygen species (ROS) such as peroxides, superoxide and singlet oxygen. These species are neutralized by a detoxification process mediated by gluthathione peroxidase and gluthathione reductase with the participation of NADPH as the reducing agent. The second phase of PPP is called the non-oxidative phase which produces a variety of sugar phosphates (Keller et al., 2014). These molecules can be further incorporated into several biosynthetic pathways like glycolysis, biosynthesis of aromatic amino acids and nucleotides (Soderberg, 2005). 


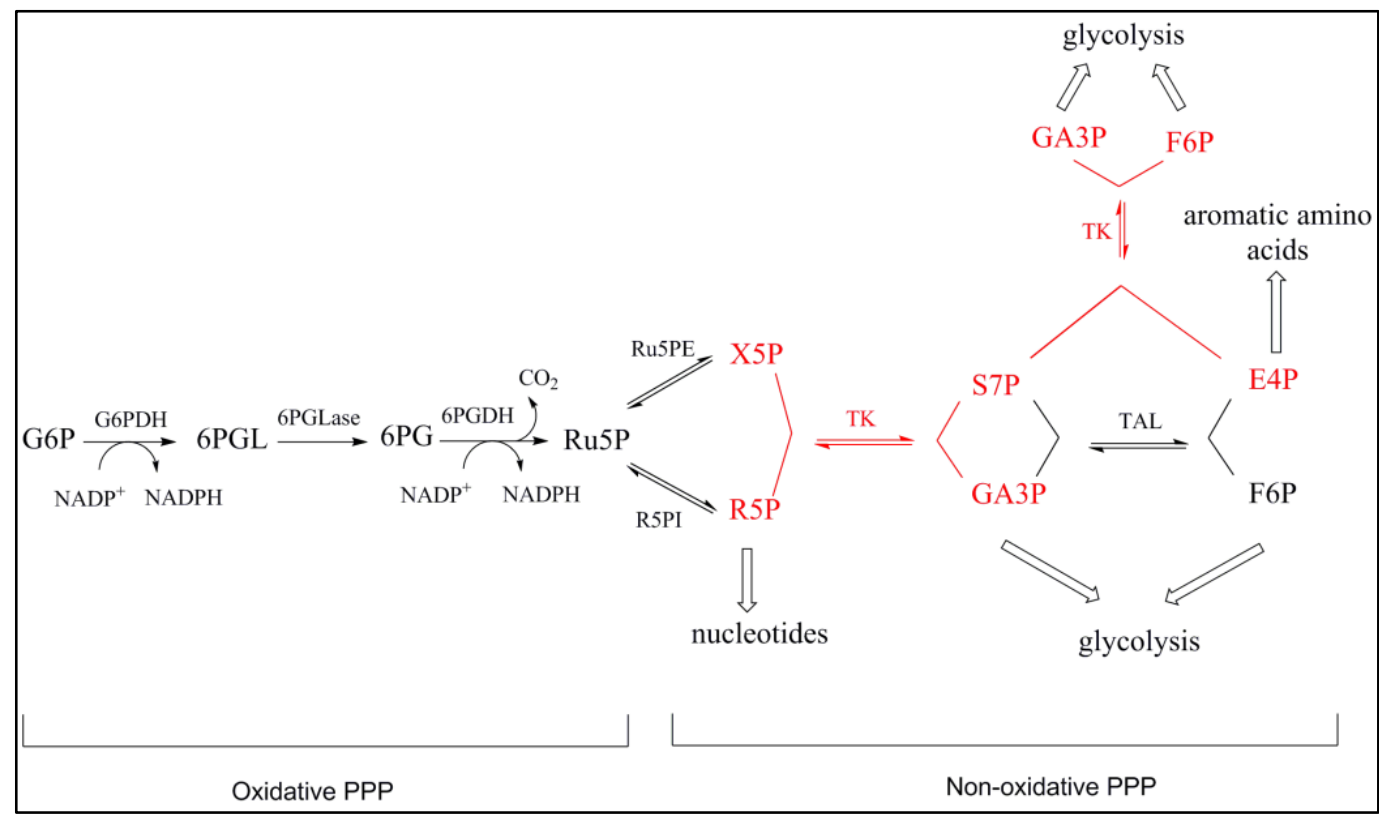

Fig 6: Pentose Phosphate Pathway and the major connection points to other metabolic pathways.

Pentose Phosphate Pathway can be divided into two parts, the oxidative part generating reducing agents NADPH and the non-oxidative part which is responsible for the sugars phosphates interconversion. The reactions catalysed by transketolases are highlighted in red. Abbreviations: G6P, glucose 6-phosphate; G6PDH, glucose 6-phosphate dehydrogenase; 6PGL, 6-phosphoglucono- $\delta$-lactone; 6GPLase, 6phosphoglucono- $\delta$-lactonase; 6PG, 6-phosphogluconate; 6PGDH, 6-phosphogluconate dehydrogenase; Ru5P, D-ribulose 5-phosphate; Ru5PE, ribulose 5-phosphate-3-epimerase; R5PI, ribose 5-phosphate isomerase; TAL, transaldolase. This figure is adapted from (Soderberg, 2005).

In addition, human TK exhibits a pivotal function in the metabolism due to its distribution in almost all the tissues (Sax et al., 1996). Several diseases were reported to have relationship with mammalian transketolase such as Alzheimer disease (Eisinger et al., 1994), diabetes (Hammes et al., 2003) and Wernicke Korsakoff syndrome (Zhao et al., 2009). However, those studies couldn't confirm any molecular basis for the observed effects. In human genome, two human TK related proteins TKTL1 and TKTL2 were revealed to have similar sequence identity to $h$ TK of $61 \%$ and $66 \%$, respectively (Mitschke et al., 2010). Although no enzymatic and cellular function have been reported for TKTL2, TKTL1 has been proposed to have linkage with metastasis and cancer patient mortality (Coy et al., 2005). Sequence alignment between $h \mathrm{TK}$ and TKTL1 reveals a major difference of 38 amino acids in the $\mathrm{N}$-terminal of the PP domain, which contains several conserved active site residues involved in cofactor and substrate binding. A "pseudo- $h$ TK" $\Delta 38$ deletion variant of human TK was generated as a TKTL1 model and Circular Dichroism and NMR studies were conducted. These results 
indicated that it is unable to bind ThDP. This study thus argues the enzymatic function of TKTL1 in the tumor cell metabolism (Schneider et al., 2012).

\subsection{Catalytic mechanism of transketolases}

Transketolases are typical ThDP-dependent enzymes and catalyse the reversible transfer of a $\mathrm{C} 2$-unit (namely 1,2-dihydroxyethyl) from ketose phosphates (donor substrate) to the $\mathrm{C} 1$ position of aldose phosphates (acceptor substrate) (Mitschke et al., 2010). Interconversion of sugar phosphates with varying carbon lengths (C3-C7) can be catalysed by transketolases. As shown in Fig 7, the whole transketolase reaction consists of two half reactions, namely the donor-half reaction (donor ligation and cleavage) and the acceptor-half reaction (acceptor ligation and product release). Identical to all ThDP-dependent enzymes, the reaction starts from the activation of the cofactor which results to the formation of the reactive ylide form of ThDP. The C2-carbanion of ThDP then attacks the carbonyl group of the donor subtrates (ketose phosphates) in a nucleophilic way to generate the first covalent intermediate (donorThDP adduct). Ionization of the $\mathrm{C} 3-\mathrm{OH}$ and the cleavage of the scissile $\mathrm{C} 2-\mathrm{C} 3$ bond of the donor-ThDP covalent intermediate leads to the formation of the next dihydroxylethyl-ThDP (DHEThDP) carbanion/enamine intermediate, which is accompanied by the release of the first product (an aldose phosphate). This central intermediate can then either react with the released aldose phosphate (reverse reaction) or attack the aldehyde group of another acceptor substrate (aldose phosphates) to form the product-ThDP covalent adduct. In the last step, one molecule of product (ketose phosphate) is released and the reaction cycle finally completes.

The catalytic mechanism of transketolase has been extensively studied using site-directed mutagenesis, protein X-ray crystallography, NMR spectroscopy and other biophysical techniques (Schellenberger, 1998; Tittmann, 2014). Asp155 of human TK was revealed to be essential for ThDP binding and divalent cation coordination which further influences the catalytic dimer formation (Wang et al., 1997). The conserved Asp477 which interacts with the C2-hydroxy group of acceptor substrates in Saccaromyces cerivisiea TK (ScTK) was investigated to be a determinant of the enantioselectivity of transketolase (Nilsson et al., 1998). X-ray crystallographic studies of $h \mathrm{TK}$ and $E c$ TK in complex with donor substrates have allowed a detailed analysis of how substrates interact with the protein and how the intermediates are stabilized at the active site of the enzymes (Asztalos et al., 2007; Lüdtke et al., 2013). One of the most astonishing findings revealed by high-resolution structures of covalent donor-ThDP intermediates is a strong out-of-plane distortion of the $\mathrm{C} 2-\mathrm{C} 2 \alpha$ bond which connects the cofactor and substrate. In addition, the scissile $\mathrm{C} 2 \alpha-\mathrm{C} 3 \alpha$ single bond was 
shown to exhibit an elongation of $0.1 \AA$ which represents a high-energy state and can reduce the barrier for the subsequent steps (Lüdtke et al., 2013; Neumann and Tittmann, 2014; Tittmann, 2014).

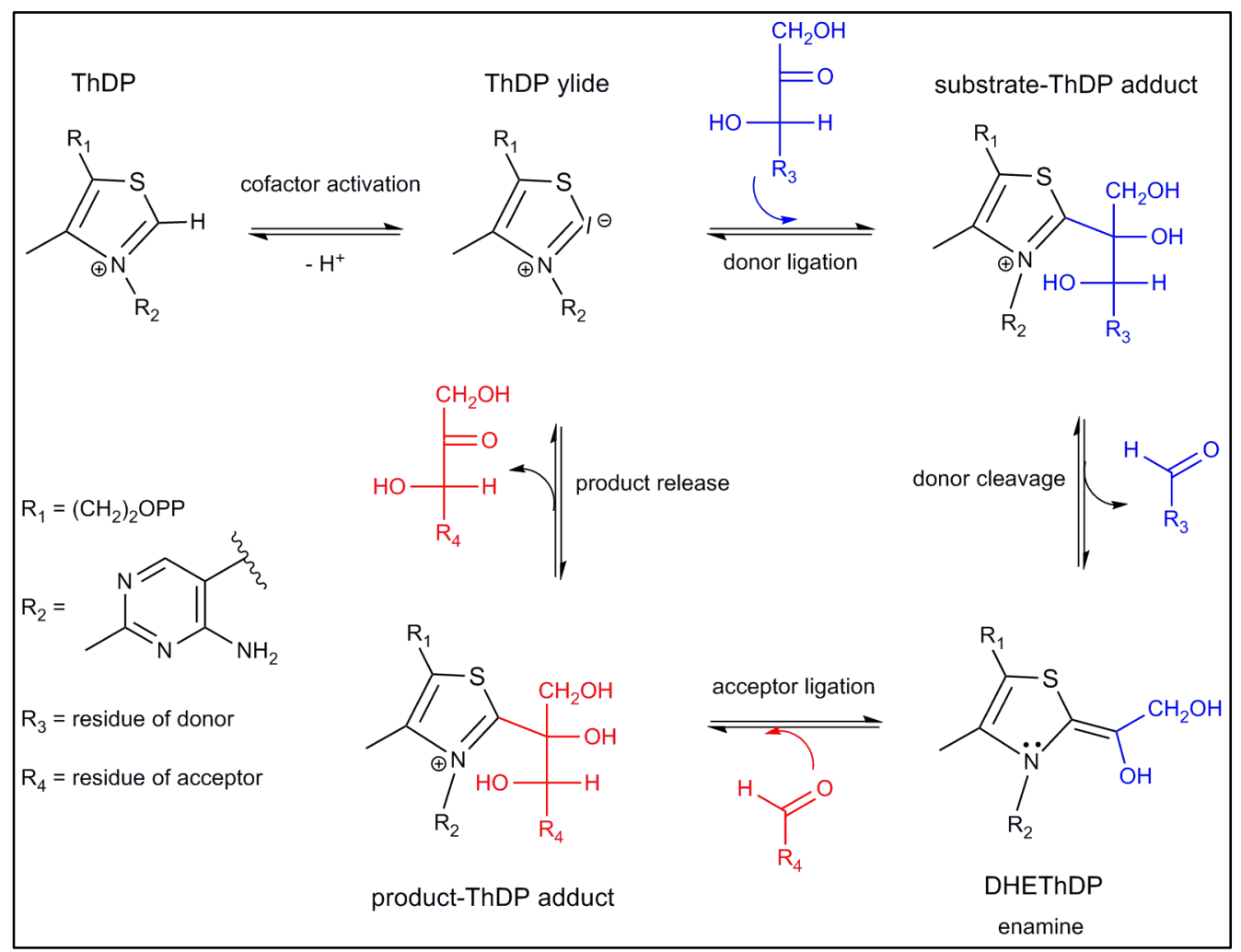

Fig 7: General catalytic mechanism of transketolase. Simplified steps and reaction intermediates are indicated. The donor-half and acceptor-half reactions are highlighted in blue and red, respectively.

\subsection{Three dimensional structure of transketolases}

The crystal structure of yeast transketolase was solved in the year of 1992 (Lindqvist et al., 1992) which represented the first structural determination of a ThDP-dependent enzyme. From that time on, structures of transketolases from several species (Gerhardt et al., 2003; Littlechild et al., 1995; Lukacik et al., 2015; Mitschke et al., 2010) were determined and those structures revealed a remarkable similarity despite that their sequences are not strictly conserved (Schenk et al., 1998). Most transketolases were crystallized in the orthorhombic form with $\mathrm{P} 2{ }_{1} 2_{1} 2_{1}$ space group, while human TK was found to be in the monoclinic form with space groups C2 and $\mathrm{P} 22_{1}$ (Lüdtke et al., 2013). The biologically active form of all transketolases were found to be homo-dimeric with active sites formed at the interface of the two neighboring subunits. The monomer of TKs shows a bean-like shape and consists of three individual $\alpha / \beta$-type domains, 
namely the N-terminal PP domain which is responsible for the binding of the pyrophosphate (anchor) of the cofactor (residues 1-276), the Pyr domain which forms interactions with the aminopyrimidine ring of ThDP, and a C-terminal domain (residues 493-623) (Mitschke et al., 2010). The function of the C-terminal domain so far is not clear, but a structural alignment with nucleotide-binding domains of other protein suggested a similar nucleotide-binding function (Schenk et al., 1998).

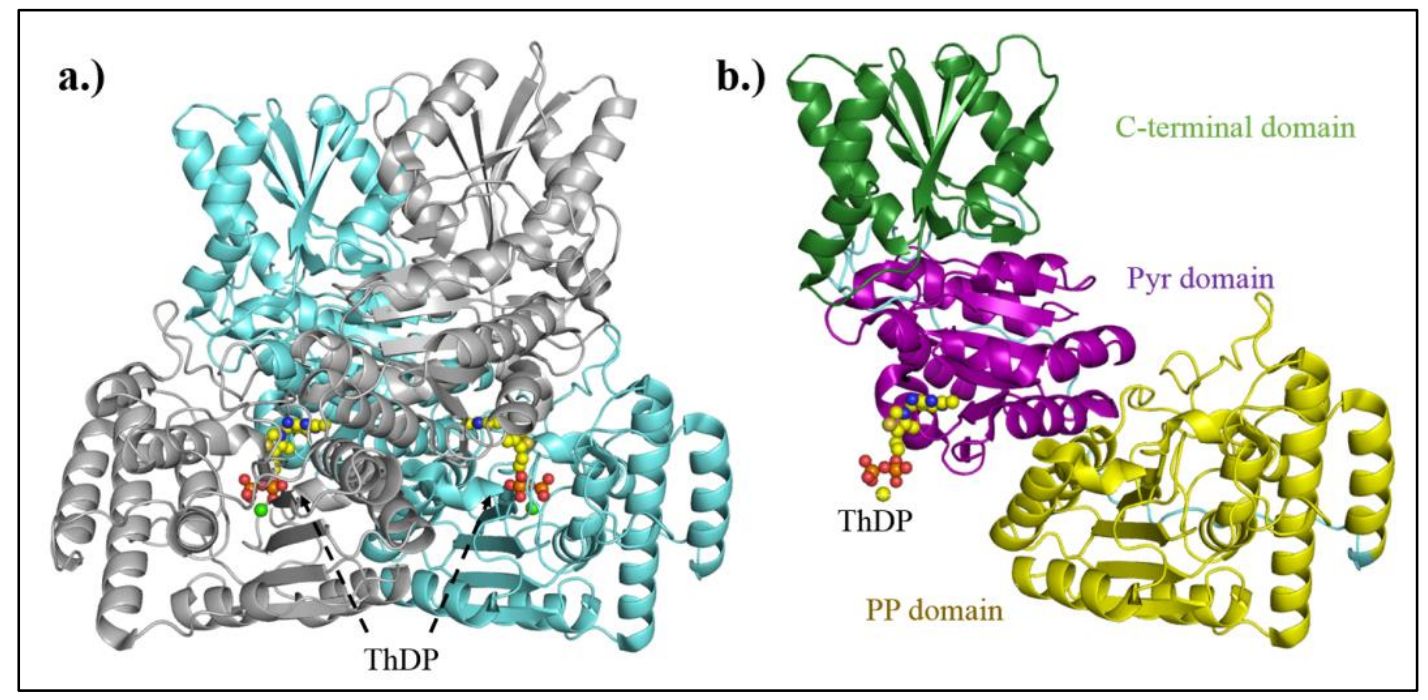

Fig 8: Three dimensional structure of human TK. a.) Crystal structure of homo-dimeric human TK (PDB code: 3MOS) (Mitschke et al., 2010) is shown in cartoon representation with the bound cofactor ThDP (yellow) and the divalent cations (green). Individual subunits are shown in cyan and grey, respectively. b.) Domain architecture of human TK monomer is shown in cartoon representation with the three domains in different colors: N-terminal PP domain (yellow), middle Pyr domain (purple) and C-terminal domain (green).

In addition, the substrate entrance site of transketolases are conserved among different species which mainly consist of several arginine, serine and histidine residues. These positively charged amino acid residues could interact with the phosphate group of the substrates and therefore position and guide the substrates into the active site for further catalysis (Schneider and Lindqvist, 1998). Other functions of the phosphate groups were proposed in enzymes such as orotidine 5 '-monophosphate decarboxylase (OMPDC) that the phosphate moiety can reduce the binding energy thus enhancing the reactivity for the so-called "substrate in pieces" reaction (Amyes and Richard, 2007; Amyes et al., 2005; Tsang et al., 2008). Now the question arises that whether this activation mechanism could also be applied to transketolases. This issue will be comprehensively studied in this thesis. 


\subsection{Application of transketolases in organic synthesis}

Since the identification of transketolase in 1953 (Racker et al., 1953) in yeast Saccharomyces cerevisiae, the application of this biologically important enzyme in the field of organic synthesis has been extensively studied (Turner, 2000). The successful overexpression of EcTK and a more recently generated His-tagged protein have made this enzyme an easily available material for large-scale production (French and Ward; Hobbs et al., 1993). The thermostability of $E c \mathrm{TK}$ also proves itself an ideal biocatalyst, therefore it can be incorporated into multienzyme synthetic experiments (Aucamp et al., 2008). The in vivo reaction of TKs, which is the reversible transfer of a $\mathrm{C} 2$-unit from ketose phosphates (donor) to the $\mathrm{C} 1$ position of aldose phosphates (acceptor), has been exploited in several synthetic strategies. But the use of the artificial substrate hydroxypyruvic acid (HPA) renders the reaction more useful as the concomitant release of $\mathrm{CO}_{2}$ results in an irreversible transformation (Srere et al., 1958).

The application of TKs in organic synthesis can be mainly divided into two approaches. The first approach is to incorporate TK into multi-enzyme synthetic routes (commonly called onepot synthesis) for the conversion of cheap, commercially available materials into valuable compounds (Guérard et al., 1999; Hecquet et al., 1996; Takayama et al., 1997). A good example is the synthesis of D-xylulose 5-phosphate (D-X5P) which is difficult to be synthesized by conventional chemical methods, but D-X5P has particular values for mechanistic studies of several enzymes and various metabolic diseases (Shaeri et al., 2008). In the one-pot synthetic approach, D-fructose 1,6-bis-phosphate undergoes the retro-aldol cleavage catalysed by fructose 1,6-bis-phosphate aldolase to give a mixture of dihydroxyacetone phosphate (DHAP) and D-glyceraldehyde 3-phosphate (GAP). GAP then serves as the acceptor substrate of EcTK to react with HPA to generate D-X5P. The equilibrium of GAP and DHAP is mediated by the use of a third enzyme named triose phosphate isomerase (TIM). The overall yield could reach $82 \%$ on a gram scale (Zimmermann et al., 1999). The second approach involves the use of saturation mutagenesis to engineer TK towards non-physiological acceptor substrates (Fig 9). A series of EcTK variants including single, double or even triple mutations were generated and can accommodate substrates such as hydroxylated substrates (Hibbert et al., 2007), aliphatic substrates (Cázares et al., 2010) and aromatic substrates (Payongsri et al., 2015). 


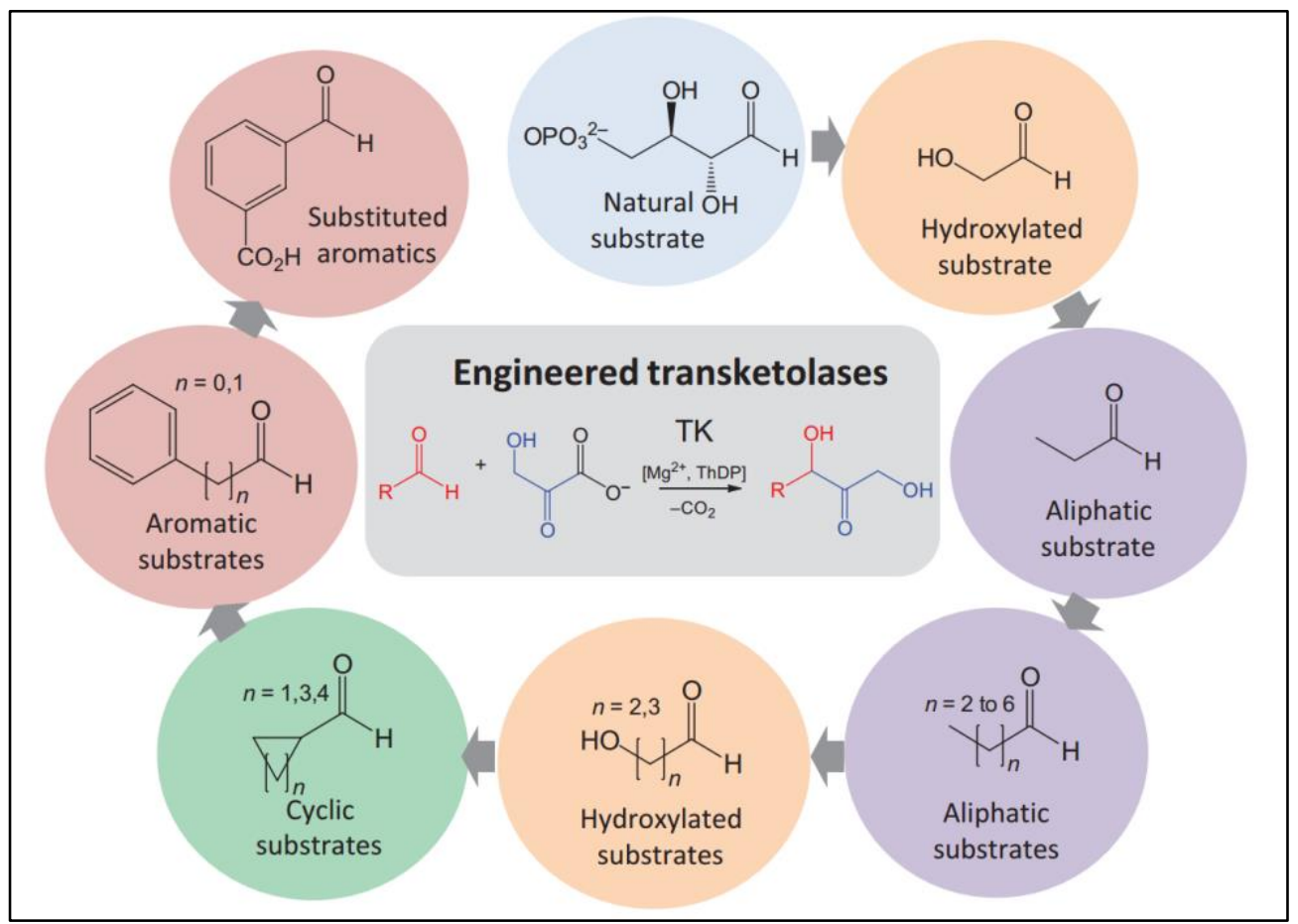

Fig 9: Range of acceptor substrates for the engineered transketolase. Figure is adapted from (Hailes et al., 2013).

\subsection{Introduction of low barrier hydrogen bonds (LBHBs)}

A low-barrier hydrogen bond (LBHB) is a special type of hydrogen bond which can occur when the $\mathrm{pKa}$ of the two heteroatoms are closely matched (Cleland and Kreevoy, 1994; Cleland et al., 1998). This allows the hydrogen to be more equally shared and this hydrogensharing leads to the formation of especially short, strong hydrogen bonds (Frey et al., 1994; Warshel et al., 2006).

Fig. 10 shows the energy profiles for different hydrogen bond types between oxygen heteroatoms. The hydrogen bond situation in water is shown in panel A, where the hydrogen clearly belongs to either the left-hand or right-hand oxygen and is more loosely bonded to the other one with a hydrogen bond length of $2.8 \AA$. Such a hydrogen bond is essentially electrostatic, and the covalent $\mathrm{O}-\mathrm{H}$ bond is usually $0.9-1.0$ A in length. Panel B represents the situation when the $\mathrm{pKa}$ of the two heteroatoms are identical and the overall O-O distance is shortened to $2.55 \AA$. Now the hydrogen can freely move between the two heteroatoms with the average position at the middle between the two oxygen atoms. This is when a LBHB forms. Panel C represents an even stronger hydrogen bond, the distance of which reduces to $2.3 \AA$ and is characterized as a single-well or short-strong hydrogen bond (Cleland et al., 1998). 


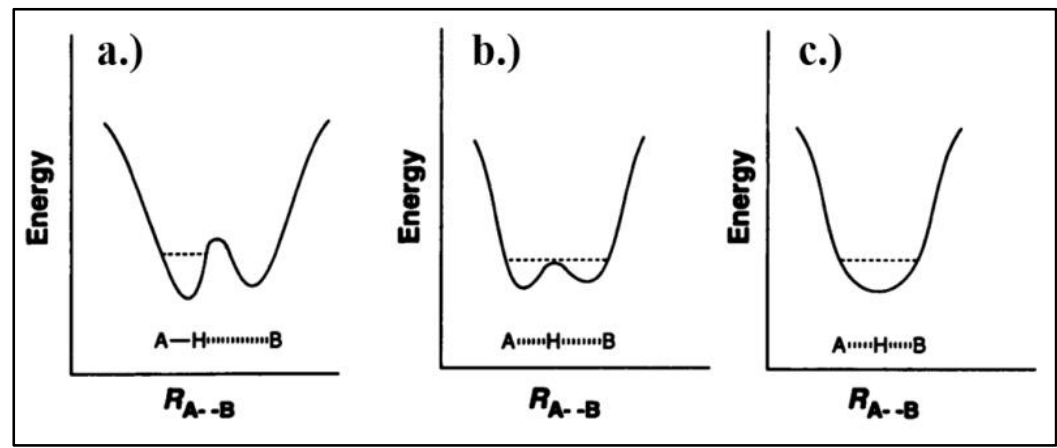

Fig 10: Energy diagrams for hydrogen bonds as a function of interatomic distance $\left(\boldsymbol{R}_{\mathrm{A}-\mathrm{B}}\right)$. a.) The two heteroatoms are separated by a weak hydrogen bond with a distance of $2.8 \AA$. The hydrogen atom is covalently bonded to atom A due to the deep and narrow energy well. b.) The two heteroatoms are separated by a strong LBHB with a distance of $2.55 \AA$. The energy barrier for the hydrogen to move between the two heteroatoms has dropped due to the strong LBHB. The average position of the hydrogen therefore lies at the center of the two heteroatoms. c.) The two heteroatoms are so close (hydrogen bond length of $2.29 \AA$ ) that the energy barrier becomes zero. In this case, the hydrogen atom bonds to the two heteroatoms in an equally covalent way. The figure is adapted from (Frey et al., 1994).

The idea that LBHBs play a role in enzymatic catalysis was first proposed in 1993 and 1994 (Gerlt and Gassman, 1993a, 1993b) and has been extensively studied during the past twenty years (Cleland, 2000; Cleland et al., 1998). Several groups have proposed that LBHBs could play a role in enzymatic catalysis by the conversion of a weak hydrogen bond in the initial enzyme-substrate complex state into a LBHB in the transition state (Du et al., 2005; Frey et al., 1994; Graham et al., 2014; Kuhn et al., 1998; Yamaguchi et al., 2009). When an enzymatic reaction involves the formation of an unstable intermediate, the transition state for forming it will also resemble it, and an ordinary hydrogen bond will be converted into a LBHB in the intermediary state. When an enzyme-inhibitor complex mimics the authentic unstable intermediate during the enzymatic reaction, metastable intermediates at the active sites will be formed and could be captured by spectroscopic and crystallographic methods which allows the observation of LBHBs (Kuhn et al., 1998; Nadal-Ferret et al., 2014; Nichols et al., 2015).

\subsection{Role of LBHBs in serine protease}

One of the most extensively studied examples of a LBHB in enzymatic catalysis is shown by serine protease family (Cassidy et al., 1997; Frey et al., 1994; Fuhrmann et al., 2006; Kuhn et al., 1998; Tamada et al., 2009). The chymotrypsin of serine protease family is characterized by a double- $\beta$ barrel fold with the catalytic triad (Asp102, His57, Ser195) located at the interface of the two domains. 


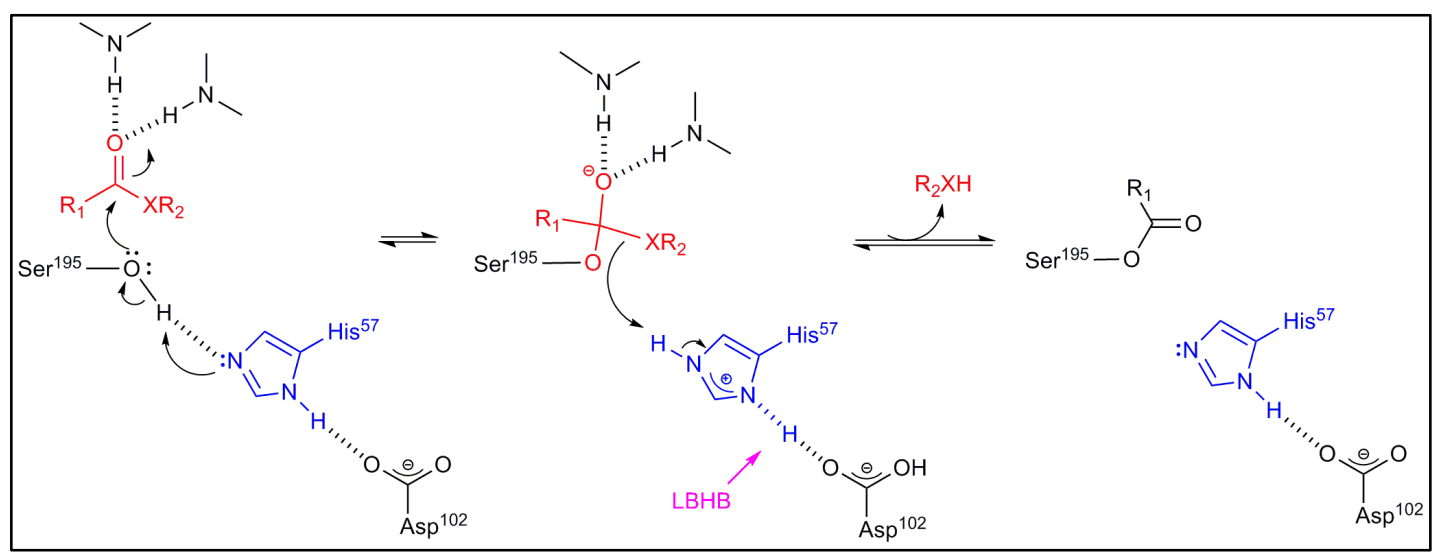

Fig 11: LBHB-facilitated general base mechanism for the acylation of chymotrypsin, the first stage of proteolytic catalysis. A LBHB (indicated by magenta arrow) is proposed to form between His57 and Asp102 in the transition state. The catalytic general base, His 57, is shown in blue and the substrate is shown in red. Figure is adapted from (Frey et al., 1994).

Fig. 11 shows a simplified mechanism for the first stage of the proteolytic catalysis, acylation. His57 first facilitates the deprotonation of Ser195 and thereby increases the nucleophilicity of the O $\gamma$ of Ser195. Then Ser195 attacks the carbonyl of the substrate's scissile bond, leading to the formation of the first tetrahedral intermediate. Frey and colleagues proposed that His57 and Asp102 form a LBHB in the transition state of the tetrahedral intermediate for acylation (Frey et al., 1994). They also hypothesized that the formation of such a higher energy bond would promote the formation of the transition state by stabilizing the Asp-His dyad and increasing the basicity of His57, thus enhancing the first step of the acylation reaction. His57 protonates the amine of the scissile bond, leading to the formation of the acyl enzyme and the subsequent release of the N-terminal portion of the cleaved substrate. The second stage of the reaction (deacylation) follows a similar mechanism which involves the participation of a catalytic water molecule. After a second meta-stable tetrahedral intermediate the $\mathrm{C}$-terminal portion of the substrate is released.

This mechanism is accepted by some but rejected by others (Ishikita and Saito, 2014). Frey and colleagues reported a low field proton at $\delta 18.3 \mathrm{ppm}$ for the proton between His57 and Asp102 of chymotrypsin in 1994 (Frey et al., 1994). In 1998, Peter and colleagues solved the crystal structure of a serine protease from Bacillus lentus and they could unambiguously observe an unusual, short hydrogen bond between the aspartic acid and the histidine in the catalytic triad (Kuhn et al., 1998). They could also locate a hydrogen atom at the middle position between the two heteroatoms. These results support the LBHB hypothesis. But in 2006, Fuhrmann and colleagues reported one X-ray crystal structure at $0.90 \AA$ A resolution of $\alpha$ lytic protease bound to the peptidyl boronic acid inhibitor, MeOSuc-Ala-Ala-Pro-boroVal, 
which mimics the transition state of the acylation reaction. They observed an ordinary hydrogen bond at the same position, which excluded the presence of the LBHB during serine protease catalysis (Fuhrmann et al., 2006). Nowadays, the role of LBHBs in serine protease is still a debated topic in the field of enzymology.

\subsection{LBHBs in phosphate binding proteins}

In addition to enzymes, examples of LBHBs in other protein families have also been observed such as the phosphate binding proteins (PBPs) (Elias et al., 2012). The molecular mechanism that bacteria can discriminate between phosphate, which is essential to life, and the chemically similar arsenate, a compound highly toxic to most organisms, has been elusive for years until the super-resolution structures of PBPs were solved. Upon phosphate binding, a LBHB was observed between $\mathrm{O} 2$ of the bound phosphate and the carboxylate of Asp62, but in the arsenate-bound structure, the short high-energy hydrogen bond undergoes a distortion which is recognized as the key component of the recognition site.

\subsection{Aim of the thesis}

The main objective of this thesis is to perform a more comprehensive study on the function of low barrier hydrogen bonds (LBHBs) in transketolase which is one of the most well-known and extensively studied ThDP-dependent enzymes. Although extensive studies from kinetic, spectroscopic and structural aspects have been performed on this enzyme, the involvement of a LBHB in the catalysis of TKs has never been studied. In this thesis, we would like to use high-resolution X-ray crystallography, site-directed mutagenesis, Circular Dichroism (CD) spectroscopy, in combination with steady-state and pre-steady-state kinetics, to comprehensively study the catalytic function of the LBHB in human TK. The concept of LBHBs playing a pivotal role during enzymatic reactions has been a controversial topic in the past twenty years (Warshel et al., 2006), therefore the main purpose of this thesis is to shed light on this topic and try to confirm if LBHBs play any role in the enzymatic catalysis.

Another key objective of this thesis is to investigate the function of the phosphate group on the native substrates of transketolase. Numerous enzymes take phosphorylated compounds as their native substrates rather than the non-phosphorylated counterparts, suggesting potential functional roles of the phosphate moiety. For transketolases, previous X-ray crystallographic studies have revealed an important role of the phosphate group for the stabilization of substrates by forming hydrogen bonding and salt bridge with amino acid side chains upon 
binding to the active site (Schneider and Lindqvist, 1998). But what functional role does the phosphate group play at different points of the reaction coordinate is still unknown and needs to be further studied. Does the phosphate group have other functions? For example, reducing the binding energy of substrates to facilitate reactions which has been proposed in several mechanistically unrelated enzymes (Kholodar et al., 2015). A question arises that whether this mechanism could also be applied to transketolases. In this thesis, the above mentioned questions will be addressed by combining X-ray crystallography, UV-Vis spectroscopy and stopped-flow technique. A more detailed picture of the function of the phosphate group shall be drawn in the end.

The third aim of this thesis is to preliminarily investigate the intrinsic linkage between protein flexibility and the enzymatic function. Previous work in our group have revealed a ring flipping phenomenon for a catalytic histidine residue in EcTK (Neumann and Tittmann, 2014) and a conformational dynamics synchronizing intermediate, water molecules, amino acid backbone and side chains in hTK (Lüdtke et al., 2013). In this thesis, several methods will be incorporated such as site-directed mutagenesis, X-ray crystallography, Circular Dichroism (CD) spectroscopy and fast kinetic techniques to investigate the relationship between these intrinsic dynamics and the enzymatic catalysis. 


\section{Materials and Methods}

\subsection{Materials}

\section{Compound}

D-fructose 6-phosphate, disodium salt hydrate

D-ribose 5-phosphate, disodium salt hydrate

D-xylulose 5-phosphate sodium salt

D-erythrose 4-phosphate, sodium salt

D-fructose

D-glyceraldehyde

D-erythrose

D-glucose

D-xylulose

D-lactose

Acid sulphur

Acetic acid

Acrylamide

Agar

Agarose

Ammonium chloride

Ammonium sulfate

\section{Supplier}

Sigma-Aldrich Chemie GmbH,

Deisenhofen

Sigma-Aldrich Chemie GmbH,

Deisenhofen

Sigma-Aldrich Chemie GmbH,

Deisenhofen

Sigma-Aldrich Chemie GmbH,

Deisenhofen

Sigma-Aldrich Chemie GmbH,

Deisenhofen

Sigma-Aldrich Chemie GmbH,

Deisenhofen

Sigma-Aldrich Chemie GmbH,

Deisenhofen

AppliChem GmbH, Darmstadt

Sigma-Aldrich Chemie GmbH,

Deisenhofen

AppliChem GmbH, Darmstadt

Carl Roth GmbH \& Co KG, Karlsruhe

Carl Roth GmbH \& Co KG, Karlsruhe

Carl Roth GmbH \& Co KG, Karlsruhe

AppliChem GmbH, Darmstadt

AppliChem GmbH, Darmstadt

Carl Roth GmbH \& Co KG, Karlsruhe

AppliChem GmbH, Darmstadt 
Ampicillin

Barium chloride, dihydrate

Benzylsulfonylfluoride (PMSF)

Calcium chloride

Coomassie Brillant blue G-250

Deuterium oxide $99.9 \%$

Diethyl ether

Dithioerythritol (DTE)

Dithiothreitol (DTT)

Dowex $50 \mathrm{Wx} 8$ cation exchanger

Ethylenediaminotetraacetic acid (EDTA)

Ethanol $(97.0 \%)$

Ethanol $(99.9 \%)$

Ethylene glycol

Glycerol (96\%)

Glycine

Glycylglycine

Hydrochloric acid (37 \%)

$\beta$-Hydroxypyruvate sodium salt (97\%)

Imidazole

Kanamycin sulfate

Magnesium chloride, hexahydrate

Magnesium sulfate

$\beta$-Mercaptoethanol

Methanol
AppliChem GmbH, Darmstadt

AppliChem GmbH, Darmstadt

AppliChem GmbH, Darmstadt

Carl Roth GmbH \& Co KG, Karlsruhe

AppliChem GmbH, Darmstadt

Sigma-Aldrich Chemie GmbH,

Deisenhofen

Carl Roth GmbH \& Co KG, Karlsruhe

AppliChem GmbH, Darmstadt

AppliChem GmbH, Darmstadt

AppliChem GmbH, Darmstadt

AppliChem GmbH, Darmstadt

Carl Roth GmbH \& Co KG, Karlsruhe

Carl Roth GmbH \& Co KG, Karlsruhe

AppliChem GmbH, Darmstadt

Carl Roth GmbH \& Co KG, Karlsruhe

Carl Roth GmbH \& Co KG, Karlsruhe

AppliChem GmbH, Darmstadt

Th. Geyer GmbH \& Co. KG, Renningen

Fluka Chemie GmbH, Switzerland

AppliChem GmbH, Darmstadt

Carl Roth GmbH \& Co KG, Karlsruhe

Carl Roth GmbH \& Co KG, Karlsruhe

Carl Roth GmbH \& Co KG, Karlsruhe

Carl Roth GmbH \& Co KG, Karlsruhe

Carl Roth GmbH \& Co KG, Karlsruhe 
Nicotinamide adenine dinucleotide, reduced (NADH)

Phenylmethylsulfonyl fluoride

Phosphoric acid (85\%)

Potassium chloride

Potassium dihydrogen phosphate

Potassium hydroxide

Polyethylene glycol (PEG) 6000

Polypropylene glycol (PPG) 400

Pyruvate

Sodium acetate, trihydrate

Sodium chloride

Sodium dodecylsulfate (SDS)

Sodium phosphite dibasic pentahydrate

Streptomycin sulfate

$\mathrm{N}, \mathrm{N}, \mathrm{N}^{\prime}, \mathrm{N}^{\prime}$-Tetramethylethylendiamin (TEMED)

Thiamin

Thiamin diphosphate (ThDP)

Trichloroacetic acid

Trimethylsilyl propionic-2,2,3,3- d4 acid, sodium salt

Tris-(hydroxymethyl)-aminomethane (Tris)

Tryptone
Sigma-Aldrich Chemie GmbH,

Deisenhofen

Sigma-Aldrich Chemie GmbH,

Deisenhofen

Carl Roth GmbH \& Co KG, Karlsruhe

Fluka Chemie GmbH, Switzerland

Carl Roth GmbH \& Co KG, Karlsruhe

AppliChem GmbH, Darmstadt

Fluka Chemie AG, Buchs, Switzerland

Fluka Chemie AG, Buchs, Switzerland

Sigma-Aldrich Chemie GmbH,

Deisenhofen

AppliChem GmbH, Darmstadt

AppliChem GmbH, Darmstadt

AppliChem GmbH, Darmstadt

Sigma-Aldrich Chemie GmbH,

Deisenhofen

AppliChem GmbH, Darmstadt

Carl Roth GmbH \& Co KG, Karlsruhe

AppliChem GmbH, Darmstadt

AppliChem GmbH, Darmstadt

Sigma-Aldrich Chemie GmbH,

Deisenhofen

Sigma-Aldrich Chemie GmbH,

Deisenhofen

AppliChem GmbH, Darmstadt

AppliChem GmbH, Darmstadt 
Yeast extract

Enyzmes, Marker and Kit systems

DNase

Plasmid prapartion QIAprep® Spin

Miniprep kit

DpnI (10 U/ $\mu \mathrm{l})$

dNTP mix (10 mM)

Lysozyme

Pfu Turbo DNA polymerase $(2.5 \mathrm{U} / \mu \mathrm{l})$

Phusion polymerase $(2 \mathrm{U} / \mu \mathrm{l})$

QuikChange site directed mutagenesis kit

Unstained protein molecular weight

standard

Gene RulerTM 1 kb DNA ladder $0.5 \mu \mathrm{g} / \mu \mathrm{l}$

6x DNA Loading Dye

Triose phosphate isomerase (TIM) +

Glycerol 3-phosphate dehydrogenase

(G3P-DH) Mix

plasmids

pGSJ427 encodes for transketolase A from

E. coli $(E c T K)$

pET28a (+) (hTK)

E. coli strains

E. coli-BL21 (DE3)

E. coli-BL21 Star (DE3)
AppliChem GmbH, Darmstadt

\section{Supplier}

AppliChem GmbH, Darmstadt

Qiagen GmbH, Hilden

Fermentas GmbH, St.Leon-Rot

Fermentas GmbH, St.Leon-Rot

AppliChem GmbH, Darmstadt

Qiagen $\mathrm{GmbH}$, Hilden or

Fermentas GmbH, St.Leon-Rot

Finnzymes, Finland

Stratagene, USA

Fermentas GmbH, St.Leon-Rot

Sigma-Aldrich Chemie GmbH,

Deisenhofen

\section{Supplier}

Provided by Prof. Dr. Georg Sprenger

(University of Stuttgart)

Novagen

\section{Supplier}

Invitrogen

Invitrogen 

E. coli-XL1-Blue
Invitrogen
E. coli-DH5 $\alpha$
Invitrogen

\section{Instruments}

ACQUITY UPLCTM System

Äkta Prime Plus

Äkta Purifier

Agilent 8453 UV-visible

spectrophotometer

Autoclave

Balance Kern EW

Balance Kern ABJ

Centrifuge Avanti J-20 XPIJA-20

Centrifuge Avanti J-30 I

Centrifuge Hettich Universal 320 R

Centrifuge tubes $(50,500$ and $1000 \mathrm{~mL})$

Circular dichroism spectrometer

Chirascan $^{\mathrm{TM}}$

\section{Chromatographic Columns \& Material}

HiPrepTM 26/10 Desalting

HisPrepTMFF 16/10

HisTrapTM Ni-NTA-Sepharose (5 mL)

SuperdexTM 200 XK 16/60

\section{Supplier}

Waters Corporation, Milford, USA

GE Healthcare, Munich

GE Healthcare, Munich

Agilent Technologies, Santa Clara, USA

Zirbus, Bad Grund

Kern, Balingen-Frommern

Kern, Balingen-Frommern

Beckmann Coulter, Krefeld

Beckmann Coulter, Krefeld

Hettich, Tuttlingen

Beckmann Coulter GmbH, Germany

Applied Photophysics Ltd., UK

GE Healthcare Bio-Sciences GmbH.

Munich, Germany

GE Healthcare Bio-Sciences GmbH.

Munich, Germany

GE Healthcare Bio-Sciences GmbH.

Munich, Germany

GE Healthcare Bio-Sciences GmbH.

Munich, Germany 
Concentrator, VivaSpin 20ml (30k, 50k,

100k MWCO), VivsSpin 6ml (30k, 50k,

Sartorius AG, Göttingen, Germany

100k MWCO)

Cover plates, $18 \mathrm{~mm}$, siliconized

Jena Bioscience, Jena, Germany

Conducting meter FiveEasy

Mettler-Toledo, Giessen, Germany

Crystallization plates (24 well) with

sealant

Hampton Research, Aliso Viejo, USA

Electrophoresis device, EV 231

Consort nv, Belgium

Fluidizer "Microfluidizer" 110S

Microfluidics, Newton, USA

HPLC/NMR 4mm syringe filter,

Pall Corporation, Port Washington, USA

(Acrodisc ${ }^{\circledR} 0.45 \mu \mathrm{m}$ nylon membrane)

or NALGENETM, Thermo Scientific

Incubator Unitron

Infors HT, Bottmingen/Basel, Switzerland

Incubator CrystalMotion

Rigaku, Kent, UK

NanoDrop2000

Thermo Scientific, Schwerte, Germany

\section{NMR-Spectrometer:}

Bruker Avance III $400 \mathrm{MHz}$ spectrometer

Bruker Biospin, Rheinstetten, Germany

DRX 400 instrument

Bruker Biospin, Rheinstetten, Germany

NMR tubes (5 mm,400 MHz)

Wilmad-LabGlass, Vineland, USA

\section{pH electrodes:}

pH meter FiveEasyTMFE20

Mettler Toledo, Giessen, Germany

pH "Minitrode"

Hamilton Company, Nevada, USA

Precision cuvettes "suprasil"

Hellma GmbH \& Co. KG, Mühlheim, Germany

SDS gel electrophoresis SE250

Hoefer, Holliston, USA 
Sterile filters, $0.20 \mu \mathrm{m}$

Superloops $(1,2,5,10,50$ and $150 \mathrm{~mL})$

Thermoblock, Digital Dry Bath 3721D

Thermomixer comfort

Thermocycler, TProfessional Standard 96 well, Gradient

UV-Vis Spectrometer, V-650

Vortex-Genie 2

Water bath Isotemp 202
VWR International GmbH, Darmstadt, Germany

GE Healthcare Europe GmbH, Freiburg, Germany

Fisher Bioblock Scientific, UK

Eppendorf AG, Hamburg, Germany

Whatman Biometra ${ }^{\circledR}$, Göttingen, German

JASCO GmbH, Gross-Umstadt, Germany

Scientific Industries, NY, USA

Thermo Scientific, Schwerte, Germany

\section{Softwares}

Gene runner V.3.05

SigmaPlot Version 11.0

Origin-7

CHIMERA

Phenix suite

PYMOL

CCP4 suite

MestreNova

MOLPROBITY

CS ChemBioDraw Ultra 12.0

Chromas 1.45
Hastings Software, Inc.

Systat Software, Inc

OriginLab Corporation, USA

http://www.cgl.ucsf.edu/chimera/

(Adams et al., 2010)

DeLano Scientific LLC

(Collaborative Computational Project, Number 4, 1994)

Mestrelab Research, Spain

http://molprobity.biochem.duke.edu/ (Davis et al., 2007)

CambridgeSoft, UK

McCarthy, C., Griffith University, Australia 
ClustalW2

ESPript 2.2

PRODRG server

Dali Search

ExPasy, ProtParam
http://www.ebi.ac.uk/Tools/msa/clustalw2/

(Larkin et al., 2007)

http://espript.ibcp.fr/ESPript/ESPript/index.php

(Gouet et al., 1999)

http://davapc1.bioch.dundee.ac.uk/prodrg/

(Schüttelkopf and van Aalten, 2004)

http://ekhidna.biocenter.helsinki.fi/dali_server/

http://web.expasy.org/protparam/

\section{Media, buffers and solutions}

Media, buffers and solutions were prepared with deionized water and autoclaved if necessary $\left(121{ }^{\circ} \mathrm{C}, 20 \mathrm{~min}\right)$. Buffers and solutions were sterilized using sterile filters with $0.45 \mu \mathrm{m}$ pore size when containing a heat-sensitive component. Buffers for usage with the Äkta systems were filtered using cellulose acetate filter with $0.20 \mu \mathrm{m}$ pore size and degassed for $20 \mathrm{~min}$. The $\mathrm{pH}$ of the solutions and buffers was adjusted by the addition of $\mathrm{NaOH}$ or $\mathrm{HCl}$ if not stated specifically.

\subsection{Methods}

\subsubsection{Molecular biology}

The plasmid pET 28a vector which encodes for $h \mathrm{TK}$ using an IPTG-induced promotor was cloned and generously provided by our previous colleague Dr. Kathrin Schröder-Tittmann. The encoded $h$ TK contains a C-terminal thrombin cleavage site followed by a hexa-histidine tag so that $h$ TK is extended by 14 amino acids ( $h$ TK-Leu-Val- Pro-Arg-Gly-Ser-Leu-Glu-HisHis-His-His-His-His) at the C-terminal.

The construct in the pGSJ427 vector which encodes EcTK was generously provided by Prof. Dr. Georg Sprenger from the University of Stuttgart. The expression of $E c$ TK does not require any exogenous inducer owing to the fact that gene of $E c \mathrm{TK}$ is under control of an intrinsically active promotor. This expression construct encodes for $E c$ TK with a C-terminal hexa-histidine tag.

Site-directed mutagenesis was used to introduce specific single mutation within the sequence of $h \mathrm{TK}$ and $E c \mathrm{TK}$. Active sites variants were generated by using the commercially available 
QuikChange-Kit (Stratagene, USA) with oligonucleotides containing the mutated codons. All utilized mutagenesis primer pairs of $h \mathrm{TK}$ and $E c \mathrm{TK}$ as well as sequencing primers are listed in Table 1.

Table 1: List of utilized mutagenesis and sequencing primers of $h \mathrm{TK}$ and $E c \mathrm{TK}$

\begin{tabular}{|c|c|}
\hline Primers & Nucleotide sequence (5'-3') \\
\hline$h$ TK_E160Q_forward & 5' GC GAA CTG AGC CAG GGC AGC GTG TGG 3' \\
\hline$h$ TK_E160Q_reverse & 5' CCA CAC GCT GCC CTG GCT CAG TTC GC 3' \\
\hline$h$ TK_E160A_forward & 5' GC GAA CTG AGC GCG GGC AGC GTG TGG 3' \\
\hline$h$ TK_E160A_reverse & 5' CCA CAC GCT GCC CGC GCT CAG TTC GC 3' \\
\hline$h$ TK_E366Q_forward & 5' GC TAT ATC GCG CAG CAG AAC ATG GTG AG 3' \\
\hline$h$ TK_E366Q_reverse & 5' CT CAC CAT GTT CTG CTG CGC GAT ATA GC 3' \\
\hline$h \mathrm{TK} \_$E165Q_forward & 5' GC AGC GTG TGG CAG GCG ATG GCG TTT GC 3' \\
\hline$h$ TK_E165Q_reverse & 5' GC AAA CGC CAT CGC CTG CCA CAC GCT GC 3' \\
\hline$h \mathrm{TK} \_\mathrm{Q} 428 \mathrm{~A} \_$forward & 5' GAT GGC CCG AGC GCG ATG GCG CTG G 3' \\
\hline$h \mathrm{TK} \_\mathrm{Q} 428 \mathrm{~A} \_r e v e r s e$ & 5' C CAG CGC CAT CGC GCT CGG GCC ATC 3' \\
\hline$h \mathrm{TK} \_S 427 \mathrm{~A} \_$forward & 5' GAT GGC CCG GCC CAG ATG GCG CTG G 3' \\
\hline$h \mathrm{TK} \_S 427 \mathrm{~A} \_r e v e r s e$ & 5' C CAG CGC CAT CTG GGC CGG GCC ATC 3' \\
\hline$h$ TK_G123A_forward & $\begin{array}{l}\text { 5' CC GAT GTG GCG ACC GCC AGC CTG GGT CAG } \\
\text { GG 3' }\end{array}$ \\
\hline$h$ TK_G123A_reverse & $\begin{array}{l}\text { 5' CC CTG ACC CAG GCT GGC GGT CGC CAC ATC } \\
\text { GG 3, }\end{array}$ \\
\hline$h \mathrm{TK} \_$H77A_forward & $\begin{array}{l}\text { 5' GTT CTG AGC AAA GGC GCT GCG GCG CCG ATT } \\
\text { CTG 3, }\end{array}$ \\
\hline$h \mathrm{TK} \_H 77 \mathrm{~A} \_$reverse & $\begin{array}{l}\text { 5' CAG AAT CGG CGC CGCAGC GCC TTT GCT CAG } \\
\text { AAC 3' }\end{array}$ \\
\hline $\begin{array}{l}h \mathrm{TK} \text { sequencing primer } \\
\text { (T7 forward) }\end{array}$ & 5' TAATACGACTCACTATAGGG 3' \\
\hline
\end{tabular}




\begin{tabular}{|c|c|}
\hline $\begin{array}{l}h \mathrm{TK} \text { sequencing primer } \\
\text { (T7 reverse) }\end{array}$ & 5' AGCTAGTTATTGCTCAGCGG 3' \\
\hline EcTK_D469N_forward & 5' C GGT CTG GGC GAA AAC GGC CCG ACT CAC C 3' \\
\hline EcTK_D469N_reverse & 5' G GTG AGT CGG GCC GTT TTC GCC CAG ACC G 3' \\
\hline EcTK_D469A_forward & 5' C GGT CTG GGC GAA GCC GGC CCG ACT CAC C 3' \\
\hline EcTK_D469A_reverse & 5' G GTG AGT CGG GCC GGC TTC GCC CAG ACC G 3' \\
\hline EcTK_H473N_forward & 5' C GGC CCG ACT AAC CAG CCG GTT G 3' \\
\hline EcTK_H473N_reverse & 5' C AAC CGG CTG GTT AGT CGG GCC G 3' \\
\hline $\begin{array}{l}\text { EcTK sequencing primer } \\
\text { (404-423 forward) }\end{array}$ & 5' CGCAGTTTAACCGTCCGGGC 3' \\
\hline $\begin{array}{l}\text { EcTK sequencing primer } \\
\text { (1203-1222 forward) }\end{array}$ & 5' GGGTAACTACATCCACTACG 3' \\
\hline $\begin{array}{l}\text { EcTK sequencing primer } \\
(602-620 \text { reverse) }\end{array}$ & 5' GCTTCGAAACGCATTGCGG 3' \\
\hline
\end{tabular}

Mutations sites are shown in bold type, the oligonucleotides were synthesized by SigmaAldrich Chemie GmbH, Deisenhofen, Germany.

\subsubsection{Concentration determination of DNA}

DNA concentrations were determined spectroscopically by measuring the absorption at 260 nm using a NanoDrop device (Thermo Scientific, Schwerte, Germany). Correlation of DNA concentrations and absorption is as follows: $1 \mathrm{~A} 260=50 \mu \mathrm{g} / \mathrm{ml}$ doubled stranded DNA $=0.15$ $\mathrm{mM}$ (in nucleotides). Normally triplecate measurements were performed and the average concentration was used for further experiments. 


\subsubsection{Site directed mutagenesis using Polymerase Chain Reaction (PCR)}

Active site variants were generated by site directed mutagenesis using Polymerase Chain Reaction (PCR). A standard PCR reaction with a total volume of $50 \mu \mathrm{L}$ contained $10 \mu \mathrm{L} 5 \mathrm{x}$ Phusion HF buffer, $1 \mu \mathrm{L}$ dNTP mix (10 mM of each nucleotide-5'-triphosphate), $2.5 \mu \mathrm{L}$ of sense and antisense primers ( $10 \mu \mathrm{M}$ dilution), $10 \mathrm{ng}(2 \mu \mathrm{L}$ of $5 \mathrm{ng} / \mu \mathrm{L})$ template DNA, $0.5 \mu \mathrm{L}$ Phusion DNA Polymerase $(2 \mathrm{U} / \mu \mathrm{L}), 1.5 \mu \mathrm{L}$ DMSO and $30 \mu \mathrm{L}$ sterile water. Addition of DMSO could reduce the formation of secondary structure of GC-rich templates thus increase the amplification of the targeted sequences. The temperature protocol included an initial denaturation step (step 1: $98{ }^{\circ} \mathrm{C}, 30 \mathrm{sec}$ ), another denaturation step (step 2: $98{ }^{\circ} \mathrm{C}, 30 \mathrm{sec}$ ), a primer annealing step (step 3: $60{ }^{\circ} \mathrm{C}, 30 \mathrm{sec}$ ), an elongation step (step 4: $72{ }^{\circ} \mathrm{C}, 5 \mathrm{~min}$ ), and a final terminal elongation step (step 5: $72{ }^{\circ} \mathrm{C}, 10 \mathrm{~min}$ ). Steps 2-4 were repeated 25 times and a lid preheat procedure to $95{ }^{\circ} \mathrm{C}$ was always used. After the PCR the mixtures were supplemented with $1 \mu \mathrm{L} \mathrm{Dpn} 1(10 \mathrm{U} / \mu \mathrm{L})$ and incubate at $37^{\circ} \mathrm{C}$ overnight to digest maternal DNA according to manufacturer's instructions (QuikChange site-directed mutagenesis kit).

\subsubsection{Agarose gel electrophoresis}

After PCR reactions DNA molecules were checked and analysed by agarose gel electrophoresis. All samples were supplemented with DNA loading dye (6 x DNA loading dye, Fermentas) and applied onto a $1 \%$ agarose gel (in TAE buffer), a DNA ladder (GeneRuler TM 1 kb DNA ladder, Fermentas) was also used as molecular weight reference. Gels were run horizontally in TAE buffer (40 mM Tris, $1 \mathrm{mM}$ EDTA, $20 \mathrm{mM}$ acetate, $\mathrm{pH} 8.5$ ) for $45 \mathrm{~min}$, and after the separation the gels were transferred into a solution containing $2 \mu \mathrm{g} / \mathrm{ml}$ ethidium bromide and stained for $10 \mathrm{~min}$. The visualization of DNA bands was carried out under UV light in a gel documentation system.

\subsubsection{DNA sequencing}

The correctness of nucleotide sequence of provided plasmids as well as the constructed variants generated by site-directed mutagenesis PCR were checked and analysed by DNA sequencing. For this purpose the DNA (600-700 ng) and $20 \mathrm{pM}$ sequencing primers (for sequencing primer see Table 1) were mixed and sterile water was supplemented to a total volume of $10 \mu \mathrm{L}$. Samples were submitted to Seqlab (Seqlab Sequence laboratories GmbH, Göttingen) and GATC (Biotech AG, Cologne) and two commercially available sequencing services were used. 


\subsubsection{Plasmid transformation}

To transfer plasmid DNA into different chemo-competent E.coli strains, the following manufacturers' protocols were used. Chemo-competent cells were prepared according to Inoue et al. and Tung et al. (Inoue et al., 1990; Tung and Chow, 1995) with minor changes and the cells were aliquoted to $50 \mu \mathrm{L}$ and stored at $-80{ }^{\circ} \mathrm{C}$ for future use. In a standard transformation procedure, $50 \mu \mathrm{L}$ of competent cells were first mixed with plasmid DNA $(1 \mu \mathrm{L}, 5 \mathrm{ng} / \mu \mathrm{L})$ and incubated on ice for $20 \mathrm{~min}$. The cells were subsequently heat-shocked at $42{ }^{\circ} \mathrm{C}$ for $45 \mathrm{~s}$ and immediately cooled on ice for $2 \mathrm{~min}$. Afterwards, $800 \mu \mathrm{L}$ of SOC medium ( $2 \%$ (w/v) trypton, $0.5 \%$ (w/v) yeast extract, $0.05 \%$ (w/v) $\mathrm{NaCl}, 2.5 \mathrm{mM} \mathrm{KCl}, 10 \mathrm{mM} \mathrm{MgCl} 2,2 \%$ (w/v) glucose) was added and cells were incubated for $1 \mathrm{~h}$ at $37{ }^{\circ} \mathrm{C}$ with shaking speed at $700 \mathrm{rpm}$ in a thermomixer. Successfully transformed cells were selected on LB-agar plates supplemented with the appropriate antibiotics. Final antibiotics concentrations in the media were $50 \mu \mathrm{g} / \mathrm{ml}$ kanamycin for human transketolase and $100 \mu \mathrm{g} / \mathrm{ml}$ ampicillin for E.coli transketolase.

\subsubsection{Protein chemistry}

\subsubsection{Concentration determination of proteins}

The protein concentration of wild type enzyme as well as all the mutants were determined according to a lab-specific method base on the protocol of Bradford (Bradford, 1976) using a colorimetric assay. Bovine serum albumin (BSA) was used as standard solution to generate a standard curve and the measurements were performed at $595 \mathrm{~nm}$. Normally triplecate measurements were performed and the average value was used for further experiments.

\subsubsection{Sodium dodecyl sulfate-polyacrylamide gel electrophoresis (SDS-PAGE)}

To check the molecular weight and purity of protein samples, SDS-PAGE was performed based on the protocol of Laemmli and Weber (Laemmli, 1970; Weber et al., 1972). SDS gels consisted of two parts, a stacking part (5\% acrylamide, $125 \mathrm{mM}$ Tris, $\mathrm{pH}$ 6.8, polymerized with $0.3 \%(\mathrm{w} / \mathrm{v})$ ammonium persulfate (APS) and $0.3 \%$ tetramethylethylenediamine (TEMED)) and a separation gel (12\% acrylamide, $375 \mathrm{mM}$ Tris, $\mathrm{pH} 8.8$, polymerized with $0.3 \%(\mathrm{w} / \mathrm{v})$ APS and $0.3 \%$ TEMED). Protein samples were first supplemented with SDS loading buffer (4 \% (w/v) glycerol, $80 \mathrm{mM}$ SDS, $150 \mathrm{mM}$ Tris, $\mathrm{pH}$ 6.8) and incubated at 95 ${ }^{\circ} \mathrm{C}$ for $5 \mathrm{~min}$, then loaded onto the stacking part of SDS gels. A protein marker was also used as molecular weight reference. Gels were run at $1 \mathrm{~mA} / \mathrm{cm}^{2}$ in SDS running buffer $(25 \mathrm{mM}$ Tris, $200 \mathrm{mM}$ glycine, $3.5 \mathrm{mM}$ SDS, pH 8.3) until the front of the loading dye reached the bottom of the gel. After protein separation was complete, the gels were stained for 20 min by 
Coomassie Blue staining solution (0.25\% (w/v) Coomassie Brilliant Blue, $30 \%$ methanol, 6 $\%$ acetic acid). Subsequently a destaining solution (30\% methanol and $10 \%$ acetic acid) was added to the gels and bleached for $30 \mathrm{~min}$ then changed to water overnight.

\subsubsection{Protein expression}

\subsection{IPTG induction of protein expression}

The expression of human, E.coli transketose and related active site mutants were basically described by Lüdtke et al. (Lüdtke et al., 2013) and Asztalos et al. (Asztalos et al., 2007). The expression plasmids pET 28a (encoding human transketolase) and pGSJ427 (encoding E.coli transketolase) were transformed into chemical competent E.coli BL21 star ${ }^{\mathrm{TM}}$ cells. With a single colony $10 \mathrm{ml} \mathrm{LB}$-medium culture was inoculated and grown at $37^{\circ} \mathrm{C}$ and $200 \mathrm{rpm}$ for $8 \mathrm{~h}$. This culture was further used to inoculate $200 \mathrm{ml} \mathrm{LB}$-medium pre-culture which was used after an overnight growth to inoculate $12 \times 1000 \mathrm{ml}$ main culture supplemented with $70 \mu \mathrm{g} / \mathrm{ml}$ thiamine in baffled shaking flasks. For expression of $h \mathrm{TK}$, when an $\mathrm{OD}_{600}$ of 0.8 was reached the temperature was rapidly decreased to $16{ }^{\circ} \mathrm{C}$ and the cultures were supplemented with 1 $\mathrm{mM}$ IPTG for the induction of the gene expression. For expression of $E c \mathrm{TK}$, cultures were cultivated at $37{ }^{\circ} \mathrm{C}$ for $12-16 \mathrm{~h}$. In all cases the cells were harvested by centrifugation (4800 rpm, $30 \mathrm{~min}, 4{ }^{\circ} \mathrm{C}$ ) and stored at $-80{ }^{\circ} \mathrm{C}$ until usage.

\subsection{Auto-induction of human transketolase}

The yield of $h \mathrm{TK}$ was pretty low ( $0.5 \mathrm{mg}$ protein out of $1 \mathrm{~g}$ cells), in order to obtain a sufficient amount of protein for a full kinetic profile, nuclear magnetic resonance (NMR) experiments as well as crystallization trials, an auto-induction method according to (Grabski et al., 2005; Studier, 2005) was applied. The basic idea of auto-induction is that certain media has the ability to induce protein expression in E.coli when the cell growth reaches saturation. In a typical auto-induction expression, a $200 \mathrm{ml}$ pre-culture was first grown overnight and was used to inoculate $12 \times 500 \mathrm{ml}$ main culture which contains $470 \mathrm{ml} \mathrm{NaCl}$-free LB-medium, 20 $\mathrm{ml}$ salt stock, $10 \mathrm{ml}$ sugar stock, $1 \mathrm{ml} \mathrm{MgSO}_{4}$ and $50 \mu \mathrm{g} / \mathrm{ml}$ kanamycin. The cells was first cultivated at $37^{\circ} \mathrm{C}$ until $\mathrm{OD}_{600}$ reached 0.8 , the temperature was then rapidly decreased to 16 ${ }^{\circ} \mathrm{C}$ to slowly induce protein expression. Bacteria will take glucose as the early energy source, later on when glucose is completely consumed glycerol will be taken as the energy source, at this stage lactose will be imported into cells and induce protein expression. The whole culture was incubated at $16{ }^{\circ} \mathrm{C}$ at $200 \mathrm{rpm}$ for $60-72 \mathrm{~h}$ before harvesting. The wet weight of the cells differed between 120 to $150 \mathrm{~g}$ per auto-induction. 


\begin{tabular}{|c|c|c|c|}
\hline \multicolumn{4}{|l|}{ Media of auto-induction } \\
\hline \multicolumn{4}{|c|}{ Main culture: $\mathrm{LB}$ medium without $\mathrm{NaCl}$} \\
\hline \multicolumn{4}{|l|}{$\mathbf{M g S O}_{4}: 1 \mathrm{M}$ stock solution } \\
\hline \multicolumn{4}{|l|}{ Stock solution of salts $(25 x)$} \\
\hline Salt & $\begin{array}{l}\text { Stock } \\
\text { concentration }\end{array}$ & Total amount $(1 \mathrm{~L})$ & Final concentration \\
\hline $\mathrm{Na}_{2} \mathrm{HPO}_{4}(141.96 \mathrm{~g} / \mathrm{mol})$ & $625 \mathrm{mM}$ & $88.73 \mathrm{~g}$ & $25 \mathrm{mM}$ \\
\hline $\mathrm{KH}_{2} \mathrm{PO}_{4}(136.1 \mathrm{~g} / \mathrm{mol})$ & $625 \mathrm{mM}$ & $85.1 \mathrm{~g}$ & $25 \mathrm{mM}$ \\
\hline $\mathrm{NH}_{4} \mathrm{Cl}(53.49 \mathrm{~g} / \mathrm{mol})$ & $1250 \mathrm{mM}$ & $66.86 \mathrm{~g}$ & $50 \mathrm{mM}$ \\
\hline $\mathrm{Na}_{2} \mathrm{SO}_{4}(142.04 \mathrm{~g} / \mathrm{mol})$ & $125 \mathrm{mM}$ & $17.76 \mathrm{~g}$ & $5 \mathrm{mM}$ \\
\hline \multicolumn{4}{|c|}{ Stock solution of sugars $(50 \times)$} \\
\hline Sugar & $\begin{array}{l}\text { Stock } \\
\text { concentration }\end{array}$ & Total amount (1 L) & Final concentration \\
\hline Glycerol (92.09 g/mol) & $25 \%(\mathrm{w} / \mathrm{v})$ & $250 \mathrm{ml}$ & $0.5 \%(\mathrm{w} / \mathrm{v})$ \\
\hline D-Glucose $(180.16 \mathrm{~g} / \mathrm{mol})$ & $2.5 \%(\mathrm{w} / \mathrm{v})$ & $25 \mathrm{~g}$ & $0.05 \%(\mathrm{w} / \mathrm{v})$ \\
\hline D-Lactose $(342.30 \mathrm{~g} / \mathrm{mol})$ & $10 \%(\mathrm{w} / \mathrm{v})$ & $100 \mathrm{~g}$ & $0.2 \%(\mathrm{w} / \mathrm{v})$ \\
\hline
\end{tabular}

\subsubsection{Protein purification of $h \mathrm{TK}$}

Typically, for one purification $100 \mathrm{~g}$ cell pellet were used as starting material and were resuspended and homogenized in $400 \mathrm{ml}$ buffer $\mathrm{A}(50 \mathrm{mM}$ Tris, $300 \mathrm{mM} \mathrm{NaCl}, 100 \mu \mathrm{M}$ ThDP, $5 \mathrm{mM} \mathrm{CaCl}_{2}, 20 \mathrm{mM}$ imidazole, $\mathrm{pH}$ 8.0) supplemented with $0.5 \mathrm{mM}$ phenylmethylsulfonyl fluoride (PMSF), $10 \mu \mathrm{g} / \mathrm{ml}$ lysozyme and $50 \mathrm{ng} / \mathrm{ml}$ DNase1. Cell disruption was performed using a Microfluidizer $110 \mathrm{~S}$ followed by a centrifugation step (45 min, $20000 \mathrm{rpm}, 4{ }^{\circ} \mathrm{C}$ ) to remove all the cell debris. The supernatant was loaded onto a Ni-NTA column equilibrated in buffer B (50 mM Tris, $300 \mathrm{mM} \mathrm{NaCl}, 20 \mathrm{mM}$ imidazole, $\mathrm{pH}$ 8.0) and a washing step was performed to remove any unspecific protein binding. The desired protein was eluted by a linear gradient over $100 \mathrm{ml}$ reaching $100 \%$ buffer C (50 mM Tris, $300 \mathrm{mM} \mathrm{NaCl}, 200 \mathrm{mM}$ imidazole, $\mathrm{pH}$ 8.0). After performing an SDS-PAGE gel electrophoresis, the fractions containing the protein of interest $(40-50 \mathrm{ml})$ were combined and concentrated to 2-3 $\mathrm{ml}$. 


\subsubsection{Protein purification of $E c T K$}

Typically, for one purification $20 \mathrm{~g}$ cell pellet were used as starting material and were resuspended and homogenized in $100 \mathrm{ml}$ buffer A (20 mM Tris, $300 \mathrm{mM} \mathrm{NaCl}, 1 \mathrm{mM}$ DTE, $1 \mathrm{mM}$ EDTA, $20 \mathrm{mM}$ imidazole, $\mathrm{pH}$ 8.0) supplemented with $0.5 \mathrm{mM}$ phenylmethylsulfonyl fluoride (PMSF), $10 \mu \mathrm{g} / \mathrm{ml}$ lysozyme and $50 \mathrm{ng} / \mathrm{ml}$ DNase1. Cell disruption was performed using a Microfluidizer $110 \mathrm{~S}$ followed by a centrifugation step (45 min, $20000 \mathrm{rpm}, 4{ }^{\circ} \mathrm{C}$ ) to remove all the cell debris. The supernatant was loaded onto a Ni-NTA column equilibrated in buffer B (20 mM Tris, $300 \mathrm{mM} \mathrm{NaCl}, 20 \mathrm{mM}$ imidazole, $\mathrm{pH}$ 8.0) and a washing step was performed to remove any unspecific protein binding. The desired protein was eluted by a linear gradient over $100 \mathrm{ml}$ reaching $100 \%$ buffer C (20 mM Tris, $300 \mathrm{mM} \mathrm{NaCl}, 200 \mathrm{mM}$ imidazole, $\mathrm{pH}$ 8.0). After performing an SDS-PAGE gel electrophoresis, the fractions containing the protein of interest $(40-50 \mathrm{ml})$ were combined and concentrated to 2-3 ml.

\subsubsection{Buffer exchange of proteins}

Different buffer systems were needed for individual experiments. To achieve this size exclusion chromatography (SEC) was applied using various columns according to the manufacturers' protocols.

For further purification of $h \mathrm{TK}$, the concentrated fractions from Ni-NTA column was applied onto a Superdex 200 column (Superdex 200 16/60, GE Healthcare) pre-equilibrated with desired buffer (for measurement purposes, $50 \mathrm{mM}$ glycylglycine, $5 \mathrm{mM} \mathrm{CaCl}_{2}, \mathrm{pH} \mathrm{7.6}$; for crystallization purposes, $50 \mathrm{mM}$ glycylglycine, $\mathrm{pH}$ 7.9) (Fig 69). After elution, an SDS-PAGE gel electrophoresis was performed to check the protein purity.

For further purification of $E c$ TK, instead of the Superdex 200 column, a high Prep-desalting column with a column volume of $50 \mathrm{ml}$ was used. Buffer systems were as same as $h \mathrm{TK}$.

\subsubsection{Kinetic methods}

\subsubsection{Steady-state activity assay}

The steady-state enzymatic activity of $h \mathrm{TK}, E c \mathrm{TK}$ wild type and related active site variants were measured spectrophotometrically by a coupled assay in which native substrates xylulose 5-phosphate (X5P) and ribose 5-phosphate (R5P) were converted into sedoheptulose 7phosphate (S7P) and glyceraldehydes 3-phosphate (GAP) and GAP was further converted to G3P by employing two auxiliary enzymes triose phosphate isomerase (TIM) and $s n$-glycerol- 
3-phosphate: NAD ${ }^{+}$2-oxidoreductase (G3P-DH) (Kochetov, 1982). Before measurement, the auxiliary enzymes which were stored as $4.8 \mathrm{M}$ ammonia sulfate sunspension had to be dialyzed overnight against $50 \mathrm{mM}$ glycylglycine, $\mathrm{pH} 7.6$ to minimize the effect of high concentration of ammonia sulfate on transketolase. In this process, NADH was concomitantly oxidized to $\mathrm{NAD}^{+}$and the reaction was monitored at $340 \mathrm{~nm}$ at certain temperatures $\left(20^{\circ} \mathrm{C}\right.$ for $h \mathrm{TK}$ and $30{ }^{\circ} \mathrm{C}$ for $\left.\mathrm{EcTK}\right)$. The assay was performed in $50 \mathrm{mM}$ glycylglycine, $\mathrm{pH} 7.6$ buffer with a total volume of $200 \mu \mathrm{l}$ and was further supplemented with $0.22 \mathrm{mM}$ NADH, 3.6 units of snglycerol-3-phosphate: $\mathrm{NAD}^{+}$2-oxidoreductase/triose-phosphate isomerase, $100 \mu \mathrm{M}$ ThDP, 5 $\mathrm{mM} \mathrm{CaCl} 2,0.1-2 \mathrm{mg} / \mathrm{ml} h \mathrm{TK}$ or $0.05-0.5 \mathrm{mg} / \mathrm{ml} E c \mathrm{TK}$, and variable concentrations of X5P and R5P. One unit is defined as the formation of $1 \mu \mathrm{mol}$ of G3P per min. In order to determine the $K_{\mathrm{m}}$ value for X5P, the concentration of R5P was kept constant at $5 \mathrm{mM}$. The $K_{\mathrm{m}}$ values for R5P was not measured due to the availability of the substrate X5P.

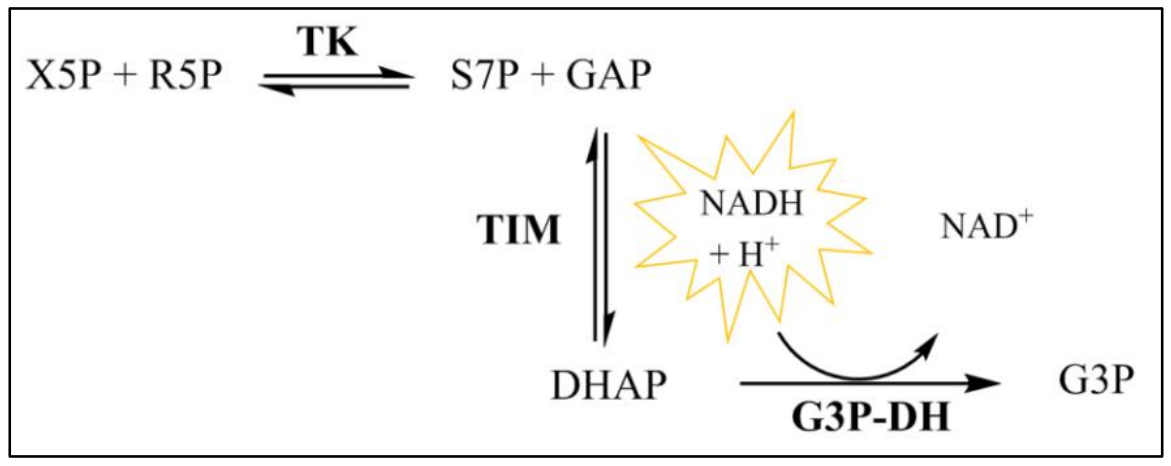

Fig 12: Simplified scheme of the steady-state activity assay of transketolase. Xylulose 5-phosphate (X5P) and ribose 5-phosphate (R5P) are converted by transketolase into sedoheptulose 7-phosphate (S7P) and glyceraldehyd 3-phosphate (GAP) which is linked to the formation of glycerol 3-phosphate (G3P) by two auxiliary enzymes (TIM and G3P-DH). The consumption of NADH is monitored spectrophotometrically at $340 \mathrm{~nm}$.

The dependence of the initial rates on the substrate concentration was analysed according to the Michaelis-Menten equation 1 or the substrate saturation equation 2.

$$
\mathrm{V}=\frac{\mathrm{Vmax} *[\mathrm{~S}]}{K_{\mathrm{M}}+[\mathrm{S}]}
$$

Equation 1: Michaelis-Menten equation. Vmax $=$ maximal velocity at substrate saturation; $K_{\mathrm{M}}=$ Michaelis constant. 


$$
\mathrm{V}=\frac{\mathrm{Vmax}}{1+\frac{K_{\mathrm{M}}}{[\mathrm{S}]}+\frac{[\mathrm{S}]}{K_{\mathrm{I}}}}
$$

Equation 2: Substrate saturation equation. Vmax $=$ maximal velocity at substrate saturation; $K_{\mathrm{M}}=$ Michaelis constant; $K_{\mathrm{I}}=$ inhibition equilibrium constant of the putative substrate inhibition.

\subsubsection{Stopped-flow kinetic methods}

Transient kinetic analysis plays an indispensable role in the understanding of working mechanism of macromolecules. For enzymes, steady-state measurements can only provide the overall catalytic efficiency and substrate specificity. In order to identify and characterize individual steps in the reaction pathway, transient or pre-steady state kinetic measurements have to be employed that is where stopped-flow and chemical-quench flow methods step in. The stopped-flow technique only has a dead time of approx. $1.5 \mathrm{~ms}$ which enables the visualization of fast kinetic events such as enzymatic intermediates formation. The single mixing and sequential stopped-flow measurement were performed with a stopped-flow spectrophotometer SX.20 MV at related wavelengths. To measure the time-resolved spectra of the intermediates depletion (AP, DHEThDP) or formation (IP, DHEThDP or enolate), a diode array detector was used and spectra were collected in equidistant time intervals. Experiments were generally performed at $4{ }^{\circ} \mathrm{C}$ for $h \mathrm{TK}$ and $25{ }^{\circ} \mathrm{C}$ for $E c \mathrm{TK}$ with on optical path length of $1 \mathrm{~cm}$. Related buffers were usually used as blank at any wavelength.

\subsection{Single mixing experiments}

In order to find the optimal wavelength for individual intermediate, UV-Vis difference spectra was measured and calculated first. The wavelengths for the single mixing stopped-flow experiments were chosen as follows. For $h \mathrm{TK}$, the depletion of AP, formation of IP and DHEThDP were measured at 325, 295 and $400 \mathrm{~nm}$, respectively. For EcTK, the AP and IP were measured at 320 and $295 \mathrm{~nm}$, respectively. A series of substrate concentrations were mixed with enzyme in a 1:1 ratio in all cases. The detailed condition regarding concentration of enzymes, cofactors, substrates and buffer systems are shown in the results part.

\subsection{Sequential mixing experiments}

In order to study whether the enzyme-bound dihydroxyethyl thiamine diphosphate intermediate (DHEThDP) derived from the non-phosphorylated substrate fructose is 
chemically active or not, a double jump (sequential) stopped-flow experiment was performed with the native acceptor substrate R5P. In the first step, $h \mathrm{TK}(8 \mathrm{mg} / \mathrm{ml})$ was mixed with donor substrate fructose $(1 \mathrm{M})$ and incubate for $500 \mathrm{~s}$ to populate DHEThDP at the active site. The optimal concentration of enzyme and substrate and the incubation time were determined by single mixing experiments. In the second mixing step, the so accumulated DHEThDP was mixed with a series of acceptor substrate R5P and measured at $308 \mathrm{~nm}$. The detailed condition regarding concentration of enzymes, cofactors, substrates and buffer systems are shown in the results part.

\subsubsection{Temperature jump ( $T$-jump) relaxation technique}

The temperature jump relaxation technique is a convenient and common method of studying rapid reversible reactions of biological macromolecules (Crooks, 1983). By employing a short pulse of electrical discharge at high voltage, the temperature of reaction sample could rise up to $10^{\circ} \mathrm{C}$ within a few microseconds, which changes the value of the initial equilibrium constant followed by relaxation to a new equilibrium at the new temperature.

In order to study the rapid proton transfer reaction of the tautomeric and ionization states of ThDP, a temperature jump experiment was applied on $h \mathrm{TK}-\mathrm{wt}(5 \mathrm{mg} / \mathrm{ml}$ in $50 \mathrm{mM}$ glycylglycine, $5 \mathrm{mM} \mathrm{CaCl}_{2}$, $\mathrm{pH}$ 7.6). Temperature jumped from $12{ }^{\circ} \mathrm{C}$ to $20{ }^{\circ} \mathrm{C}$ and the reaction was measured by UV-Vis spectroscopy at $325 \mathrm{~nm}$ and $294 \mathrm{~nm}$ for the AP and IP form, respectively. The thereof obtained progress curves were fitted either by a single exponential equation which is used for a one-step reaction or by a double exponential equation used for a two-step reaction. Samples used for T-jump experiments could be recovered and after spinning down aggregates, protein could be reused for other purposes.

\subsubsection{Biophysical methods}

\subsubsection{Circular Dichroism (CD) spectroscopy}

CD spectroscopy measures the differential absorption of left- and right-handed circularly polarised light which arise due to chiral optical activity. When cofactor ThDP, which processes pro-chiral centres, is bound to the active site of transketolase, it becomes optically active and then can be analysed by CD spectroscopy.

Previous studied (Nemeria et al., 2007) have shown that different enzyme-bound ThDP gives rise to characteristic $\mathrm{CD}$ signals which allows the discrimination of individual chemical states of the aminopyrimidine ring: the 4'-amino form (AP), the 1', 4'-imino form (IP), and the 
aminopyrimidinium form $\left(\mathrm{APH}^{+}\right)$. AP form has a negative $\mathrm{CD}$ signal at 320-330 nm while IP form gives rise to a positive signal at $295-305 \mathrm{~nm}$. For the $\mathrm{APH}^{+}$form, although there is no $\mathrm{CD}$ signature of this species reported in the literatures so far, our previous colleagues have observed a negative $\mathrm{CD}$ signal at around $290 \mathrm{~nm}$ which most likely to be the $\mathrm{APH}^{+}$form.

\subsection{Active site titration of $E c$ TK}

After purification step, EcTK contains no cofactor and has to be supplemented with extra ThDP to form the active holo-enzyme. In order to compare the cofactor binding capacity between wild type enzyme and different active site mutants, a CD spectroscopy based cofactor titration experiment was performed. In a typical experiment, $200 \mu 12.0 \mathrm{mg} / \mathrm{mL}$ enzyme in 50 $\mathrm{mM}$ glycylglycine, $\mathrm{pH} 7.6$ buffer supplemented with $5 \mathrm{mM} \mathrm{CaCl}_{2}$ was used as the starting point for the titration and by adding ThDP $(100 \mathrm{mM})$, a series of ThDP concentrations were achieved and measured. Measurements were conducted using the following parameters:

- Temperature: $25^{\circ} \mathrm{C}$

- Time-per-point: $2.0 \mathrm{sec}$

- Wavelength: 250-400 nm

- Bandwidth: $1 \mathrm{~nm}$

- Step: 0.5

- Cuvette: $10 \mathrm{~mm}$

\subsection{Analysis of tautomeric forms of $\mathrm{ThDP}$ at different $\mathrm{pH}$ values}

According to the Brønsted-Lowry acid and base theory, when AP or IP form acquires a proton the conjugated acid $\mathrm{APH}^{+}$form will be generated and these three species should exist in the following equilibrium: $\mathrm{APH}^{+} \rightleftharpoons \mathrm{AP}+\mathrm{IP}$. In order to study the intrinsic protonic equilibria of the enzyme bound ThDP of wild type enzyme and different active-site variants, a $\mathrm{pH}$ titration experiment was performed according to (Lüdtke et al., 2013). In a typical experiment 2.0 - 2.5 $\mathrm{mg} / \mathrm{ml}$ enzyme in $20 \mathrm{mM}$ glycylglycine, $20 \mathrm{mM}$ MES buffer at a starting $\mathrm{pH}$ around 4.8 was titrated with Tris base (1M, $\mathrm{pH} 11)$ to get a series of $\mathrm{pH}$ values until basic $\mathrm{pH} 9$. Due to the fact that $E c$ TK is purified as apo-enzyme, exogenous ThDP $(100-300 \mu \mathrm{M})$ was supplemented and incubated for $30 \mathrm{~min}$ to reconstitute the holo-enzyme, while $h \mathrm{TK}$ needs no additional cofactor. For each $\mathrm{pH}$ value, $200 \mu \mathrm{l}$ of protein was taken and measured by $\mathrm{CD}$ spectrometer using a $10 \mathrm{~mm}$ cuvette. Measurements were conducted using the following parameters:

- Temperature: $20^{\circ} \mathrm{C}(h \mathrm{TK}), 25{ }^{\circ} \mathrm{C}(E c \mathrm{TK})$

- Time-per-point: $2.0 \mathrm{sec}$

- Wavelength: 250-400 nm

- Bandwidth: $1 \mathrm{~nm}$ 
- Step: 0.5

- Cuvette: $10 \mathrm{~mm}$

\subsection{Analysis of substrate and ligand binding}

In order to study the effect of different substrates and ligands binding, CD spectra were recorded before and after the addition of substrates or ligands. To clearly see the changes, difference spectra were calculated accordingly. In a typical experiment, $2.0 \mathrm{mg} / \mathrm{ml}$ enzyme in $50 \mathrm{mM}$ glycylglycine $5 \mathrm{mM} \mathrm{CaCl}_{2}$ at $\mathrm{pH} 7.6$ was recorded first then supplemented with various substrates and ligands. The final concentration of substrates or ligands and the incubation time are shown in Table 2.

Table 2: Substrates and conditions for ligand binding CD measurement.

\begin{tabular}{ccc}
\hline Substrate or ligand & Final concentration & Incubation time \\
\hline F6P & $5 \mathrm{mM}$ & $1 \mathrm{~min}$ \\
HPA & $20 \mathrm{mM}$ & $20 \mathrm{~min}$ \\
Fructose & $25 \mathrm{mM}$ & $20 \mathrm{~min}$ \\
Xylulose & $25 \mathrm{mM}$ & $1 \mathrm{~min}$ \\
Phosphite & $5 \mathrm{mM}$ & $2 \mathrm{~min}$ \\
Phosphate & $5 \mathrm{mM}$ & $2 \mathrm{~min}$ \\
\hline
\end{tabular}

\subsubsection{Analysis of covalent reaction intermediates by acid quench ${ }^{1} \mathrm{H}-\mathrm{NMR}$ spectroscopy}

In order to identify the covalent reaction intermediates of each microscopic step during ThDPdependent enzyme catalysis, a NMR based acid quench method was developed recently and has been applied on several enzymes (Kluger and Tittmann, 2008; Tittmann et al., 2003). NMR fingerprints of individual intermediate like the substrate-ThDP covalent adduct, the after cleavage carbanion/enamine intermediate have been confirmed by using chemically synthesized ThDP analogues. In the case of TK, this method allows to specify which intermediates accumulate at the active site and by using the integration function of MestreNova, the distribution of accumulated intermediates could be calculated.

In a typical experiment, $250 \mu \mathrm{l}$ of $15 \mathrm{mg} / \mathrm{ml}$ enzyme $(219 \mu \mathrm{M}$ active sites $h \mathrm{TK}$ or $208 \mu \mathrm{M}$ active sites $E c$ TK) were mixed with $250 \mu 1$ of $50 \mathrm{mM}$ substrate in $50 \mathrm{mM}$ glycylglycine $5 \mathrm{mM}$ 
$\mathrm{CaCl}_{2}$ at $\mathrm{pH} 7.6$ and generally incubated for $1 \mathrm{~min}$ at $6{ }^{\circ} \mathrm{C}$ if not mentioned elsewhere. The reaction was stopped by the addition of $250 \mu \mathrm{l}$ of $12.5 \%$ (w/v) trichloroacetic acid, $3.7 \%$ (v/v) $\mathrm{HCl}$ in $\mathrm{D}_{2} \mathrm{O}, \mathrm{pH} 0.75$. The samples were gently mixed and incubated for 5 min at $6{ }^{\circ} \mathrm{C}$ followed by a centrifugation step ( $30 \mathrm{~min}, 15000 \mathrm{rpm}, 4{ }^{\circ} \mathrm{C}$ ) to remove the denatured enzymes. The clear supernatant containing the acid-stable cofactor and substrate-cofactor adducts was filtered ( $0.45 \mu \mathrm{m}$ pore size) and measured by a NMR spectrometer by Prof. Dr. Kai Tittmann. As the internal standard, 3-(trimethylsilyl) propionic-2,2,3,3-d4 acid was used. For suppression of the water signal, a pre-saturation technique was used. All spectra were analysed by MestreNova and Sigmaplot.

\subsubsection{X-ray Crystallography}

X-ray diffraction datasets, collected at different synchrotron radiation facilities (ESRF, France; DESY, Hamburg; SLS, Switzerland), were processed with XDS (Kabsch, 2010). Protein models were iteratively refined and improved with PHENIX (Adams et al., 2010) and Coot (Emsley et al., 2010) and finally validated with an online program MOLPROBITY (Davis et al., 2007).

\subsection{Crystallization}

Single crystals were obtained by the vapor-diffusion method in hanging and sitting drops according to previously reported crystallization conditions from Asztalos et al. (Asztalos et al., 2007) for $E c$ TK, Mitschke et al. (Mitschke et al., 2010) for $h$ TK and Lüdtke et al. (Lüdtke, 2012; Lüdtke et al., 2013) for $E c$ TK and $h$ TK. In order to improve the quality of crystals, macroseeding was performed for both $E c \mathrm{TK}$ and $h \mathrm{TK}$.

In a typical crystallization set up, $h \mathrm{TK}$ was concentrated to $12-18 \mathrm{mg} / \mathrm{ml}$ in $50 \mathrm{mM}$ glycylglycine (pH 7.9) and supplemented with $1 \mathrm{mM}$ ThDP and $5 \mathrm{mM} \mathrm{CaCl}_{2}$. After an incubation on ice for $20 \mathrm{~min}, 3 \mu \mathrm{l}$ of protein solution was mixed in a 1:1 ratio with a reservoir solution containing 13-15.5\% (w/v) PEG 6000, 4 \% PPG 400, 2 \% glycerol, 50 mM glycylglycine at $\mathrm{pH}$ 7.9. Crystals grown in the hanging drops were adhered to a strong protein skin formed on the top of the drop, which made the crystal fishing very difficult. In order to solve this problem, sitting drop method was later on used. Crystals of $h$ TK should appear in one or two weeks at $6-8{ }^{\circ} \mathrm{C}$.

To reproducibly obtain single crystals of $E c \mathrm{TK}$, the protein was first concentrated to 16-20 $\mathrm{mg} / \mathrm{ml}$ in $50 \mathrm{mM}$ glycylglycine (pH 7.9) and supplemented with $5 \mathrm{mM} \mathrm{ThDP}$ and $5 \mathrm{mM} \mathrm{CaCl}_{2}$. After an incubation on ice for $20 \mathrm{~min}$ the holo enzyme was reconstituted. $3 \mu \mathrm{l}$ of protein solution was mixed in a 1:1 ratio with a reservoir solution containing 17-22\% (w/v) PEG 
6000, $2 \%$ glycerol, $50 \mathrm{mM}$ glycylglycine at $\mathrm{pH}$ 7.9. Crystals of EcTK should appear in three or four weeks at $6-8{ }^{\circ} \mathrm{C}$.

For both $h \mathrm{TK}$ and $E c \mathrm{TK}$ crystallization plates were first set up at $6-8{ }^{\circ} \mathrm{C}$. After $24 \mathrm{~h}$ macroseeding was performed to improve the crystal quality. Previously obtained crystals were first transferred into 1-2 $\mu \mathrm{l}$ of reservoir solution with middle range PEG concentration and crushed with a fine needle. Seeds of micro-sized crystals were transferred into each new drop by using a horse hair or a needle. The wells were sealed again by circular cover sides or a transparent tape for hanging drop plates and sitting drop plates, respectively.

Table 3: Conditions for substrate soaking experiments and data collection

\begin{tabular}{|c|c|c|}
\hline Structure & Soaking conditions & Resolution (§) \\
\hline$h$ TK-E160Q & $10 \mathrm{~s}$ in cryo-protectant & 1.04 \\
\hline$h \mathrm{TK}-\mathrm{E} 160 \mathrm{Q}+$ fructose 6-phosphate & $1 \mathrm{~min}, 100 \mathrm{mM}$ F6P & 1.06 \\
\hline$h$ TK-E366Q + xylulose 5-phosphate & $1 \mathrm{~min}, 100 \mathrm{mM}$ X5P & 1.5 \\
\hline$h \mathrm{TK}-\mathrm{wt}+$ fructose & $20 \mathrm{~min}, 200 \mathrm{mM}$ fructose & 1.5 \\
\hline$h \mathrm{TK}-\mathrm{wt}+$ xylulose & $5 \mathrm{~min}, 200 \mathrm{mM}$ xylulose & 1.5 \\
\hline$h \mathrm{TK}$-wt + phosphite & $3 \mathrm{~min}, 200 \mathrm{mM}$ phosphite & 1.5 \\
\hline$h \mathrm{TK}-\mathrm{H} 77 \mathrm{~A}+$ fructose 6-phosphate & $1 \mathrm{~min}, 100 \mathrm{mM}$ F6P & 1.9 \\
\hline$h \mathrm{TK}-\mathrm{S} 427 \mathrm{~A}+$ fructose 6-phosphate & $1 \mathrm{~min}, 100 \mathrm{mM}$ F6P & 1.25 \\
\hline$h \mathrm{TK}-\mathrm{Q} 428 \mathrm{~A}+$ fructose 6-phosphate & $1 \mathrm{~min}, 100 \mathrm{mM}$ F6P & 1.5 \\
\hline EcTK-D469N apo-enzyme & $10 \mathrm{~s}$ in cryo-protectant & 1.2 \\
\hline EcTK-D469N holo-enzyme & $10 \mathrm{~s}$ in cryo-protectant & 1.06 \\
\hline EcTK-D469A & $10 \mathrm{~s}$ in cryo-protectant & 1.15 \\
\hline
\end{tabular}

\subsection{Crystal harvest and substrate soaking}

The crystals were first incubated in a cryoprotectant solution for 5-10 s before flash-cooling in liquid nitrogen. For $h$ TK the solution contains $10 \%$ (w/v) PEG 6000, $5 \%$ (v/v) PEG 400 and $15 \%$ ethylene glycol in $50 \mathrm{mM}$ glycylglycine, $5 \mathrm{mM}$ ThDP and $5 \mathrm{mM} \mathrm{CaCl}_{2}(\mathrm{pH}$ 7.9). 
For EcTK the solution contains $20 \%$ (w/v) PEG 6000 and $30 \%$ (v/v) ethylene glycol in 50 $\mathrm{mM}$ glycylglycine, $5 \mathrm{mM}$ ThDP and $10 \mathrm{mM} \mathrm{CaCl}_{2}(\mathrm{pH}$ 7.9).

To trap intermediates within crystals, substrate soaking experiments were performed. The cryoprotectant solution was first supplemented with substrate and the $\mathrm{pH}$ had to be adjusted to 7.9 if necessary. Single crystals were transferred into the soaking solution and incubated for a defined time at $6-8{ }^{\circ} \mathrm{C}$. Individual soaking conditions for all structures presented in this thesis are listed in Table 3. All crystals were stored in liquid nitrogen until measurement.

\subsection{Crystal testing in-house}

Prior to data collection at synchrotrons, the diffracting properties and overall quality (mosaicity, twining, resolution etc.) of crystals were first tested in house in a nitrogen cryostream (130-140 K, XSTREAM2000, Rigaku/MSC, Japan) with an R-AXIS IV++ imaging-plate system (Rigaku/MSC, Japan) using $\mathrm{CuK} \alpha$ radiation (wavelength $1.5418 \AA$ ) generated by a Rigaku MM-007 rotating-anode generator.

\subsection{Data collection at the synchrotron}

Crystals with high diffraction quality were sent to different synchrotron sources (European Synchrotron Radiation Facility (ESRF) in Grenoble, France; German Electron Synchrotron (DESY) in Hamburg, Germany; Swiss Light Source (SLS) in Villingen, Switzerland) and all the high resolution datasets were collected based on a method described below.

The needle- or rod-like shaped crystals were first fished along the loop axis with half of the length or even more sticking out of the loop in order to reduce the statistical noise from crystal mounting materials, loop, excess cryoprotectant and the mother liquor. Up to $0.15 \AA$ A resolution enhancement could be achieved when the dataset is collected outside the mounting loop. To get a full completeness, first we collected medium resolution (1.4 -1.6 $\AA$ ) dataset with short exposure time for the full completeness inside the loop. The outside portion was used to collect high resolution data set. The high and low resolution datasets were afterwards merged with XDS (Kabsch, 2010). In order to reduce the effects of radiation damage arising from brilliant beams at synchrotrons, datasets of several spots with a separation of at least 1.5-2 times of beam size along the loop axis were collected. Merge of datasets with best resolution and statistics was later on performed by XDS and used for structure refinement.

\subsection{Data processing}

Datasets were generally processed and scaled with XDS with the help of Dr. Piotr Neumann (Department of Molecular Structural Biology, University of Göttingen). For the estimation of the highest resolution limit, standard rules with $\mathrm{I} /$ sigma (I) $\sim 2$ and $\mathrm{R}_{\text {merge }} \sim 50 \%$ were used for 
the determination of where to truncate the data. Data collection and structure refinement statistics can be found in the appendix.

\subsection{Model building, refinement and validation}

Our previous co-workers had reported that $h \mathrm{TK}$ could be crystallized in two monoclinic space groups $\mathrm{C} 2$ and $\mathrm{P} 21$ by using the same condition. In this study, we have obtained $h \mathrm{TK}$ wt and active sites variants crystal structures with the same space group.

Structure refinement was carried out using PHENIX by employing the maximum likelihood method with $5 \%$ of randomly chosen data for validation by the $\mathrm{R}_{\text {free }}$ factor. After a first rigid body refinement against medium resolution $(2.5 \AA)$, constrained refinements were automatically performed against higher resolution data in steps of $0.2 \AA$ by PHENIX. A water picking cycle was performed before the model was inspected manually with COOT in which the conformation of side chains and the position of water molecules or other ligands were checked. Atomic anisotropic parameters (ADPs) were refined in the next cycle for the detection of "anomalies" in thermal motions. The positions of hydrogen atoms were added with PHENIX.REDUCE (Adams et al., 2010) in the next cycle and refined using the riding model. Crystallographic Information File (CIF) for ligands (buffer molecules, substrates and cofactors) for refinement with PHENIX were generated with the PRODRG server (Schüttelkopf and van Aalten, 2004). Validation of the refined models was carried out using COOT (Emsley et al., 2010) and an online server MOLPROBITY (Davis et al., 2007) . Sigma A-weighted electron density maps ( $2 \mathrm{mFo}-\mathrm{DFc}, \mathrm{mFo}-\mathrm{DFc})$ were generated with PHENIX (Adams et al., 2010). Preparation of figures was carried out with PYMOL. 


\section{Results}

\subsection{Observation and characterization of a low barrier hydrogen bond (LBHB) in $h$ TK}

\subsubsection{Capture of a low barrier hydrogen bond (LBHB) in human TK wild type}

The existence of LBHBs has been a controversial topic in the field of enzymological research since the proposal that LBHBs play a role during enzymatic catalysis was first put forth by Frey, P. A. and his colleagues in 1993 and 1994 (Frey et al., 1994; Gerlt and Gassman, 1993a, 1993b). During the past two decades, numerous studies based on spectroscopic, computational and crystallographic results on several enzymes have gradually increased the acceptance among biochemists of the concept of LBHBs in enzymatic catalysis (Cleland and Kreevoy, 1994; Klinman, 2015; Kuhn et al., 1998; Oltrogge and Boxer, 2015). However, the specific function of LBHBs during enzymatic reactions is still not known. Previous colleagues in our group have solved the crystal structures of human TK in complex with its physiological substrates X5P, F6P and S7P at ultra-high resolution $(\sim 1 \AA)$, which provided superior models to locate hydrogen atoms in the structure of $h$ TK (Lüdtke et al., 2013).

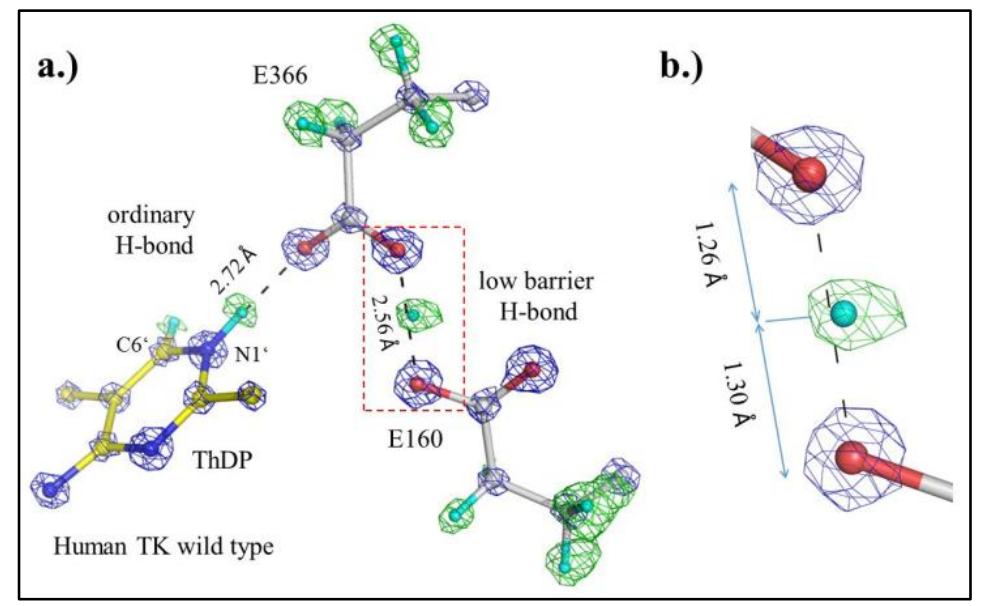

Fig 13: A low barrier hydrogen bond (LBHB) in the crystal structure of human TK wild type in complex with physiological substrate X5P at $0.97 \AA$. a.) The LBHB is observed between Glu160 and Glu366 from neighbouring subunit with a hydrogen bond distance about $2.56 \AA$. The aminopyrimidine ring of ThDP, Glu160 and Glu366 are shown as ball and stick representation and surrounded by the calculated $2 \mathrm{mFo}-\mathrm{DFc}$ map (contoured at $5 \sigma$ level, blue). The corresponding hydrogen atoms are shown as spheres in cyan surrounded by a mFo-DFc map (contoured at $3 \sigma$ level, green). An ordinary hydrogen bond with a distance of $2.72 \AA$ between N1' and E366 is also shown for comparison. b.) Detailed view of the LBHB captured in the structure of $h \mathrm{TK}$ wild type. The distances between the shared hydrogen atom and the hydrogen bond donor/acceptor atoms are highlighted. The observed hydrogen atom nearly locates in the middle of the hydrogen bond. 
By careful reanalysis of these well-defined structures, a short hydrogen bond of $2.56 \AA$, which lies in the regime of LBHBs, was unexpectedly found between the conserved Glu366 and another Glu160 from neighbouring subunit (Fig 13). A strong electron density between Glu160 and Glu366 in the mFo-DFc map (contoured at $3 \sigma$ level, green) clearly indicates the presence of a hydrogen atom and the modelled hydrogen atom locates at nearly equidistant position from the hydrogen bond donor and acceptor. At the covalent donor-ThDP stage of transketolase catalysis, the six-membered aminopyrimidine ring is proposed to exist as the IP form (Lüdtke et al., 2013), therefore extra positive electron density was observed near the N1' atom for the covalently bound hydrogen atom. The hydrogen bond between N1' of ThDP and E366 was determined as an ordinary bond with a distance of $2.72 \AA$.

Human TK exists as a functional dimer with each active site containing one cofactor. The two molecules of ThDP are separated by around 20 Å but interact directly by a "proton wire" which is composed of six acidic amino acid residues (E366, E160, E165 and E366', E160', E165'), two basic amino acid residues (Q367, Q367') and several water molecules (Fig 14).

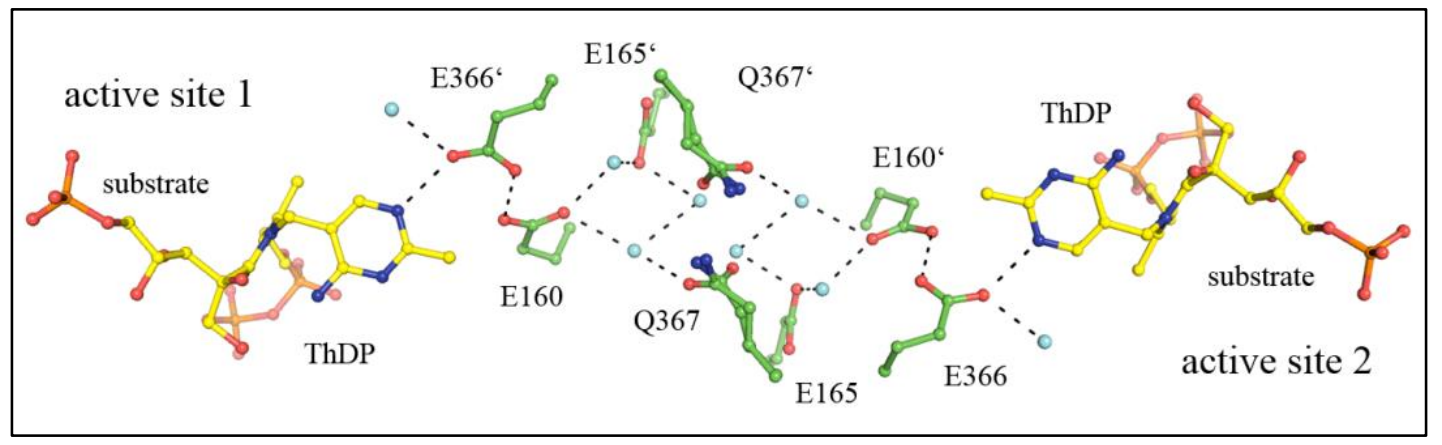

Fig 14: Active site communication in human TK. Active site communication between the two monomers of human TK mediated by amino acid side chains and several water molecules. The X5PThDP covalent adduct intermediates are shown as ball-stick representation in yellow and amino acid side chains in green. Water molecules are shown as spheres in cyan. Hydrogen bonding interactions $(<$ $3.0 \AA$ ) are shown as dashed line in black.

\subsubsection{Structure of $h T K-E 160 Q$ in complex with F6P reveals the replacement of the LBHB by an ordinary H-bond}

In order to study the catalytic function of the LBHB, the E160Q variant of human TK was generated and the crystal structure was determined to $1.06 \AA\left(\mathrm{R}_{\text {work }}=11.12 \%, \mathrm{R}_{\text {free }}=13.05\right.$ $\%$ ) in complex with physiological substrate F6P. The resolution is slightly lower than that of the wild type structure but the quality is enough for locating hydrogen atoms at the active site. The architecture of the proton wire "tunnel" shows no obvious difference except for the single mutation site. A glutamine is found at the position of glutamate in the wild type and the amide 
function forms a hydrogen bond with the oxygen atom of the carbonyl group with a distance of $2.80 \AA$ which lies in the regime of ordinary hydrogen bonds (Fig 15). The two hydrogen atoms on the amide group are well defined at the ideal positions and the hydrogen atom between the $\mathrm{N}$ of Gln160 and the $\mathrm{O}$ of Glu366 clearly belongs to the amide group of Gln160, again confirming the presence of an ordinary hydrogen bond. The position of E366 undergoes a minor adjustment, leading to the formation of a short hydrogen bond (around $2.62 \AA$ ) between the N1' atom of aminopyrimidine and E366. The positive electron density observed near the N1' atom confirms the conclusion that IP form populates at the donor-ThDP intermediate stage during catalysis, and also exclude the possibility of a LBHB for this hydrogen bond. Therefore, an ordinary hydrogen has been introduced into human TK and replaced the LBHB in the wild type between Glu160 and 366.

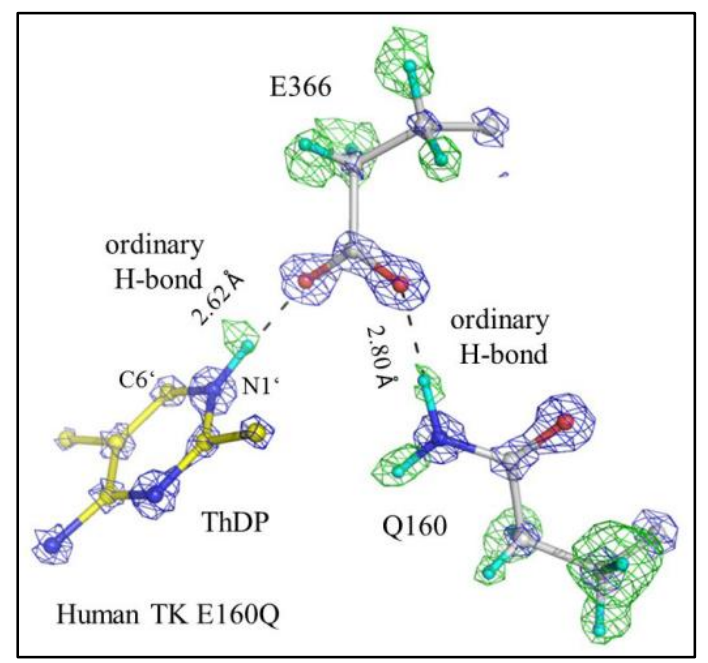

Fig 15: An ordinary H-bond is observed in the crystal structure of $h \mathrm{TK}$ E160Q in complex with donor substrate F6P. Capture of an ordinary hydrogen bond instead of a LBHB in $h$ TK-E160Q variant. Amino acid residues Glu366, Gln160 and ThDP are shown in ball-stick representation surrounded by a $2 \mathrm{mFo}-\mathrm{DFc}$ map (contoured at $5 \sigma$ level, blue). The corresponding hydrogen atoms are shown as spheres in cyan surrounded by a $\mathrm{mFo}-\mathrm{DFc}$ map (contoured at $2.5 \sigma$ level, green). The ordinary hydrogen bond between E366 and Q160 is highlighted with interatomic distance.

Closer inspection of the electron density around the two residues forming the LBHB in wild type and ordinary hydrogen bond in E160Q variant reveals different protonation states (Fig 16). In the wild type structure where the LBHB is observed, the electron density for all atoms on Glu160 and Glu366 are well defined so that the negative charge are delocalised on both glutamates. The shared hydrogen locates at the middle position of these two glutamates. However, in the structure of E160Q variant, side chains of Gln160 and Glu366 exhibits diffused electron density properties, clearly suggesting that these two residues are present as the protonated form. The introduction of Gln160 disrupts the matched $\mathrm{pKa}$, which is a crucial criteria for formation of LBHBs, leads to the relaxation of energy and an ordinary hydrogen bond thus formed. 


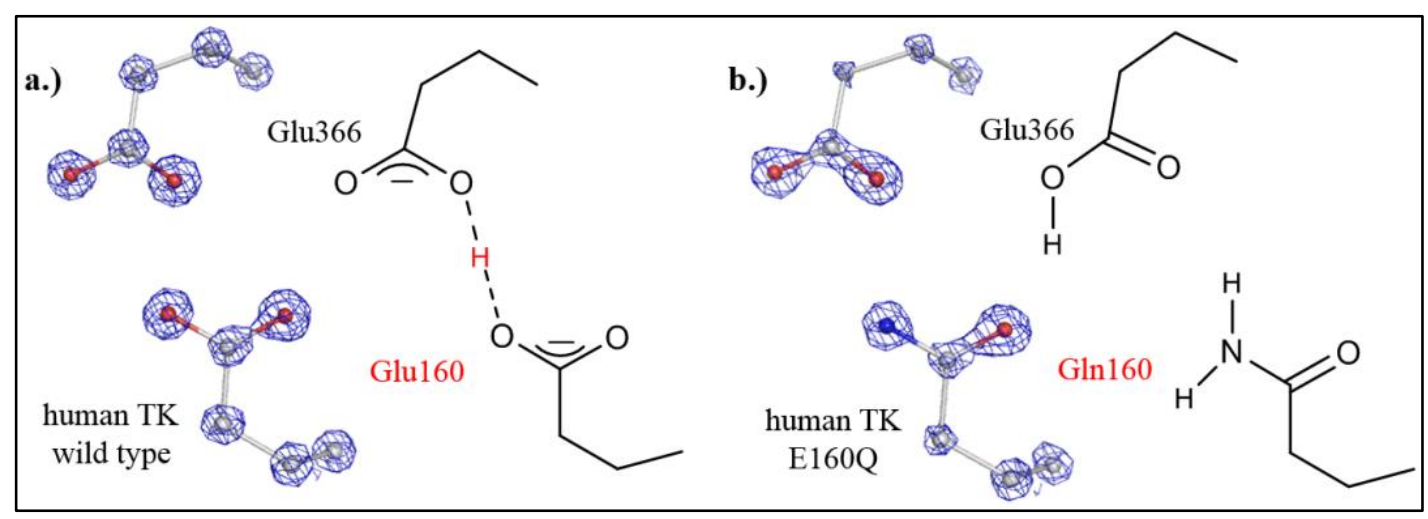

Fig 16: Detailed view on the chemical structure of the protonation state for LBHB-relative amino acid residues. a.) Side chain of Glu160 and 366 in human TK wild type. b.) Side chain of Gln160 and Glu366 in human TK E160Q.

\subsubsection{Crystal structure evidence of the elongated scissile bond and distorted covalent intermediate in $h$ TK E160Q}

For $h \mathrm{TK}$ and $E c \mathrm{TK}$ wild type, previous colleagues of our group had successfully captured the covalent donor-ThDP intermediates and made the extraordinary observation that the newly formed $\mathrm{C}-\mathrm{C}$ bond exhibits a strong angular distortion compared with the thiazolium ring system (Asztalos et al., 2007; Lüdtke et al., 2013). An elongated scissile C-C single bond had also been noticed in $h \mathrm{TK}$ when high resolution crystal structures were determined (Lüdtke et al., 2013). These physical distortions in high energy state are stabilized by the protein's conformation from relaxation, thereby could reduce the energy barrier for the subsequent steps.

Interestingly, identical physical distortions have also been observed in the structure of $h \mathrm{TK}$ E160Q variant. As shown in Fig 17, the C-C single bond that links the C2 of substrate F6P $(\mathrm{C} 2 \mathrm{x})$ and the $\mathrm{C} 2$ on the thiazolium ring of cofactor ThDP is not in the plane defined by the thiazolium ring. The $\mathrm{C} 2$ atom of the thiazolium ring is thought to be sp2 hybridized based on the knowledge that the thiazolium is aromatic, therefore the newly formed covalent bond with F6P should be coplanar with the thiazolium. However, this bond is out of the plane by about $17^{\circ}$ which is similar to that of the wild type (about $22^{\circ}, 24^{\circ}$ and $21^{\circ}$ in X5P, F6P and S7P respectively) (Lüdtke et al., 2013). Closer inspection of the five-membered thiazolium ring reveals the S1, N3, C4 and C5 atoms are almost arranged in one plane except for the reactive $\mathrm{C} 2$ atom which is slightly out-of-plane. In addition, the C4M (methyl group) and C6 (methylene group) also exhibit out-of-plane properties, which in together with the $\mathrm{C} 2$ atom, leads to a slightly bent ring system of the thiazolium moiety. 


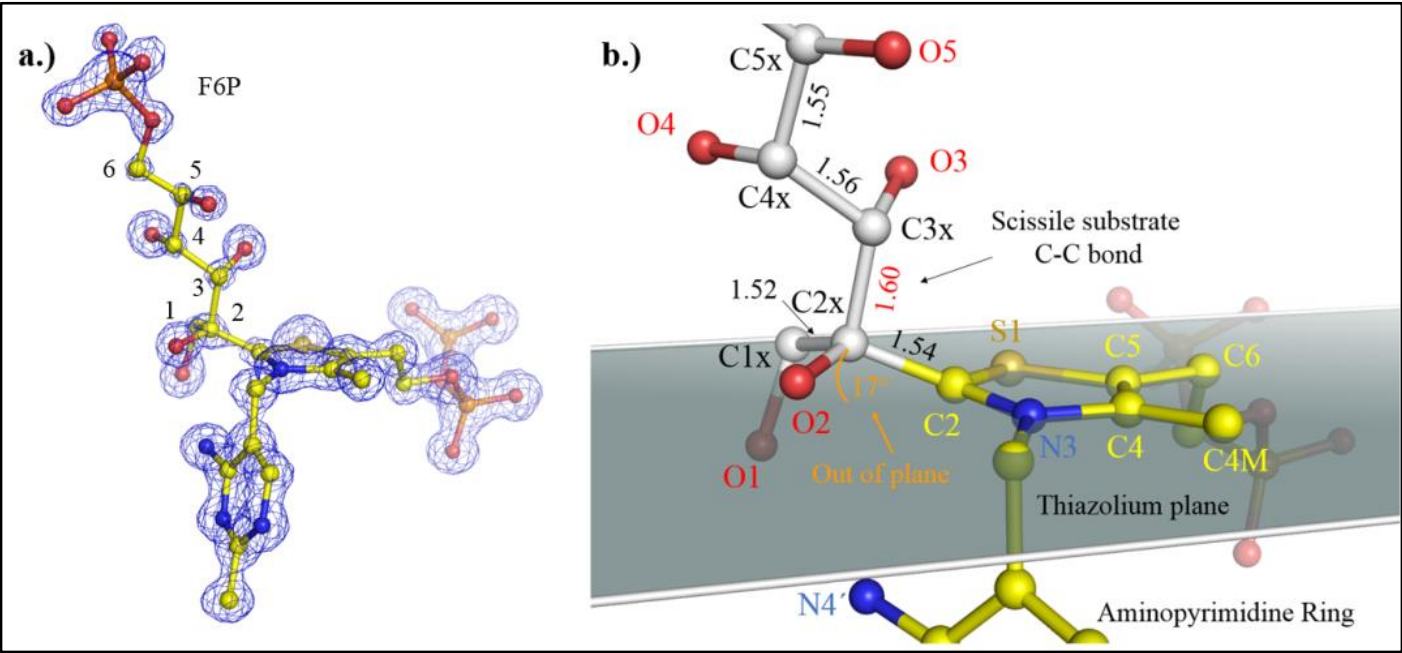

Fig 17: Observation of the elongated scissile bond and physically distorted covalent intermediate in $h$ TK E160Q in complex with F6P. a.) Crystal structure of the covalent F6P-ThDP intermediate at the active site of $h \mathrm{TK}$ E160Q variant with the corresponding 2mFo-DFc electron density map (blue, contoured at $1.5 \sigma$ level) in stereo view. The carbon atoms on the substrate F6P are labelled. b.) Closeup view of the intermediate that highlights the elongated scissile bond (C2x-C3x) and the out-of-plane distortion of the $\mathrm{C} 2-\mathrm{C} 2 \mathrm{x}$ bond relative to the ring plane of the aromatic thiazolium ring (atoms N3, C4 and C5 are used for the generation of the plane and is shown in grey). Lengths of carbon-carbon bonds $(\AA)$ are indicated and the elongated scissile $\mathrm{C} 2 \mathrm{x}-\mathrm{C} 3 \mathrm{x}$ bond is highlighted in red.

Analysis of the atomic B-factors of the enzyme bound F6P-ThDP intermediate reveals a higher flexibility of the substrate moiety compared with the cofactor ThDP (Fig 18). In addition, the thiazolium ring also exhibits increased $\mathrm{B}$-factors relative to other parts of ThDP such as the aminipyrimidine ring and the diphosphate moiety. These results suggest that covalent binding of the donor substrate F6P to the cofactor ThDP would induce a higher flexibility of the fivemembered thiozolium ring, which might account for the formation of the distorted ring system. The enzyme bound substrate F6P forms multiple hydrogen bonding interactions with active site residues as well as a molecule of ethylene glycol (EDO) binding with the phosphate group which comes from the cryo-protectant. Most of the hydrogen interactions of the substrate with the active site residues are very similar to that of the same F6P-ThDP intermediate in the structure of E.coli TK (Asztalos et al., 2007). 


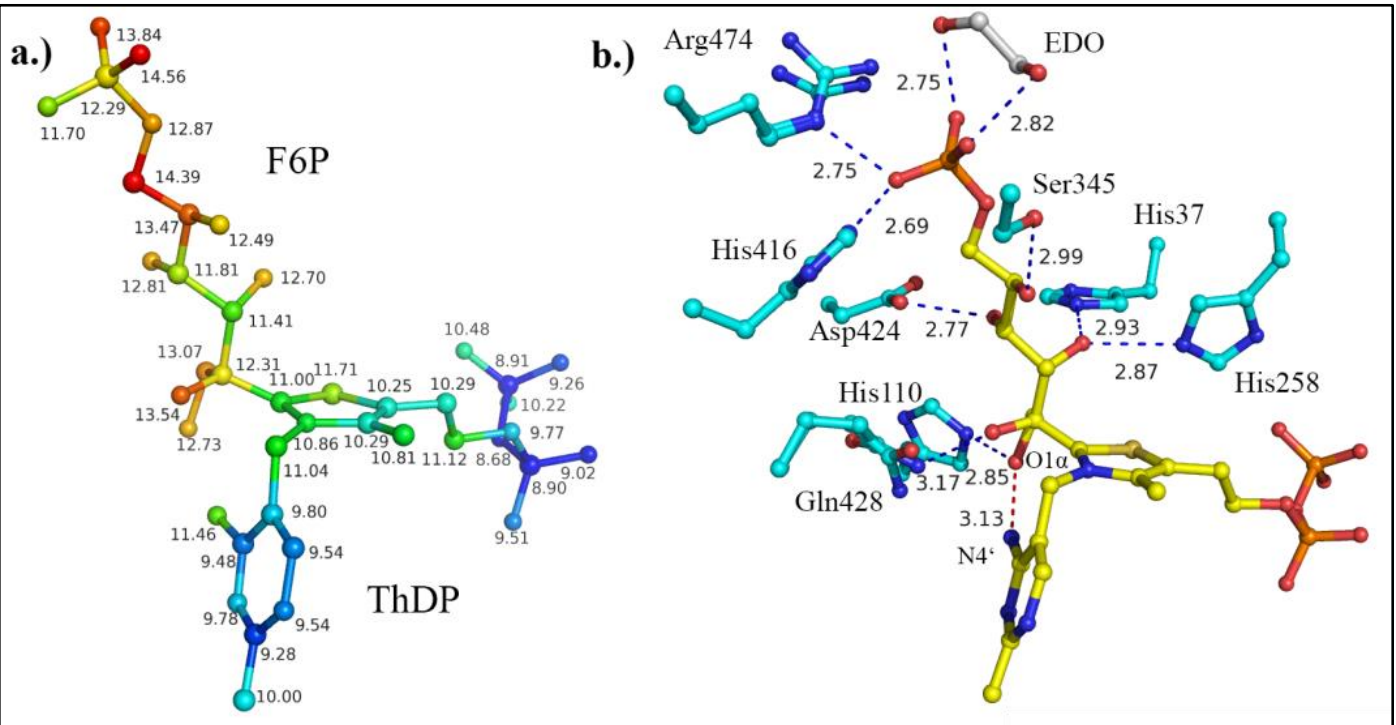

Fig 18: Active site interactions with the F6P-ThDP intermediate. a.) Atoms of the F6P-ThDP adduct are colour-labelled according to their individual B-factors with most mobile atoms in red $\left(\approx 15 \AA^{2}\right)$ and less mobile ones in blue $\left(\approx 9 \AA^{2}\right)$. b.) Amino acid side chains that are involved in the binding and positioning of the covalently bound F6P-ThDP intermediate are shown in cyan with the intermediate shown in yellow. One ethylene glycol (EDO) molecule binds closely to the phosphate group of F6P is shown in grey. Hydrogen bonding interactions are highlighted as blue dashed lines with interatomic distances (in $\AA$ ). Please note that the distance between the O1 $\alpha$ atom on the hydroxyl group of sugar and the N4' of the AP ring is highlighted in red with a distance of $3.13 \AA$.

\subsubsection{Crystal structure of $h$ TK-E160Q at resting state}

The crystal structure of human TK E160Q variant at the ground state has also been determined to $1.04 \AA$ with reasonable statistics $\left(\mathrm{R}_{\text {work }}=11.26 \%, \mathrm{R}_{\text {free }}=13.14 \%\right)$. A structural alignment of the E160Q variant at the ground state with the structure in complex with substrate F6P revealed an RMSD (using all 617 residues per chain, and $99 \%$ of sequence in PDBeFold) for the monomer of $0.42 \AA$. This indicates very little structural change resulting from the substrate binding. Analysis of the active site residues where the LBHB is observed in wild type reveals similar but slightly different structural architecture compared to that of the E160Q variant in complex with F6P (Fig 19). The hydrogen bond length between Gln160 and Glu366 is now around $2.81 \AA$ which is a typical ordinary hydrogen bond as in the structure in complex with F6P. On the other side of the Glu366, the N1' atom of the aminopyrimidine forms a hydrogen bond with the Glu366 with a distance of $2.66 \AA$, which is $0.04 \AA$ longer than that in the structure in complex with F6P. This minor change could be contributed to the effect of substrate binding. Unfortunately, the hydrogens on the Gln160 are not visible although the resolution of the structure is already high enough for detecting hydrogen atoms. 


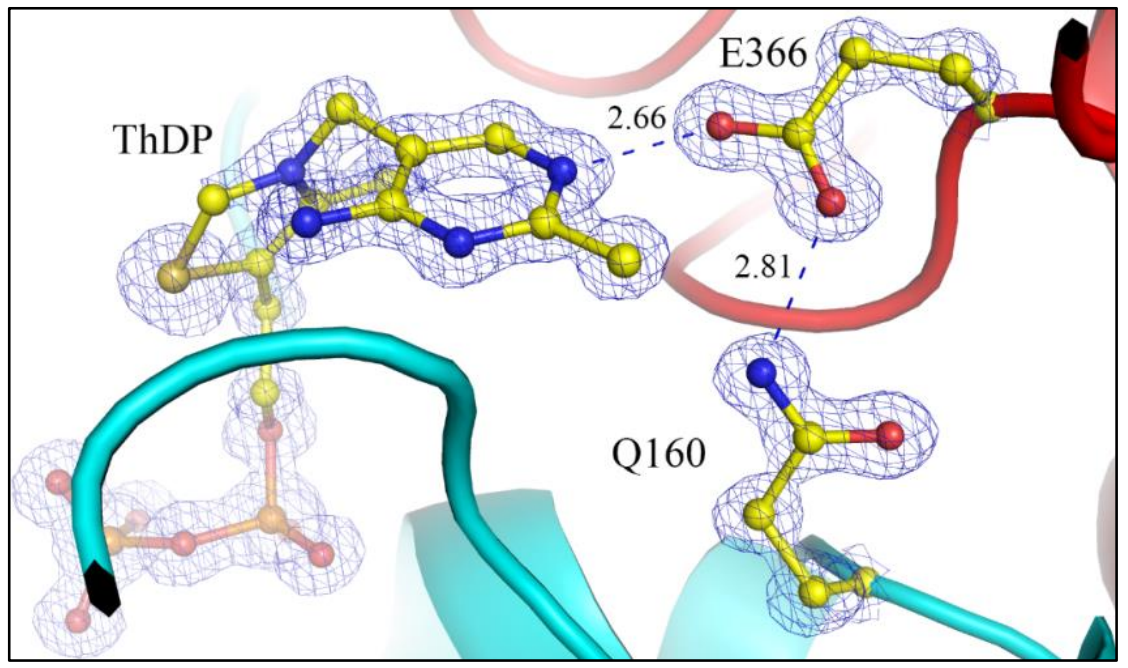

Fig 19: Active site architecture of $\boldsymbol{h}$ TK E160Q at resting state. Cofactor ThDP, residues Gln160 and Glu366 are shown in ball-stick representation surrounded by a $2 \mathrm{mFo}-\mathrm{DFc}$ map (contoured at $1.5 \sigma$ level, blue). The hydrogen interactions between N1' of ThDP and carbonyl of E366 as well as the oxygen atom of hydroxyl on E366 and nitrogen of the amide on E160 are indicated by blue dashed line with inter-atomic distances. Two monomers forming the active site are shown in cartoon representation in red and cyan, respectively.

\subsubsection{Capture X5P at the docking site in the structure of $h$ TK E366Q variant}

The structure of $h$ TK E366Q active site variant in complex with donor substrate X5P could be determined to medium resolution $\left(1.5 \AA, \mathrm{R}_{\text {work }}=15.58 \%, \mathrm{R}_{\text {free }}=18.65 \%\right)$. The strong positive electron density peaks in the $\mathrm{mFo}-\mathrm{DFc}$ map in close proximity to the cofactor ThDP could be unambiguously interpreted as the donor substrate X5P existing in its acyclic keto-form (Fig 20). The electron density fits to the ligand molecule quite well and no traces of the hydrate form of the phosphor-sugar can be detected. Detailed analysis of the atomic B-factors revealed the ligand X5P possesses higher values compared with its surrounding active site residues as well as the cofactor ThDP, indicating relatively higher flexibility of the bound X5P. Closer inspection of the atomic B-factors of X5P itself suggests a lower flexibility of the phosphate moiety than the sugar part, which might arise from the stronger hydrogen bonding interaction with phosphate moiety (each oxygen atom has at least three $\mathrm{H}$-bonding). The reactive $\mathrm{C} 2$ atom on thiazolium ring also shows higher flexibility compared with other parts of ThDP, strongly indicating that binding of donor substrate would activate ThDP. The bound X5P forms multiple hydrogen bonding interactions with its neighbouring amino acid residues (His37, 258, 416, Arg474 and 318, Ser345, Asp424 and Gln428) as well as several water molecules and the conformation of $\mathrm{X} 5 \mathrm{P}$ is pre-organized for the subsequent nucleophilic reaction with the 
cofactor. The inter-atomic distance between the reactive center $\mathrm{C} 2$ atom on thiazolium ring of ThDP and the C2 carbonyl atom of donor substrate is $3.63 \AA$.

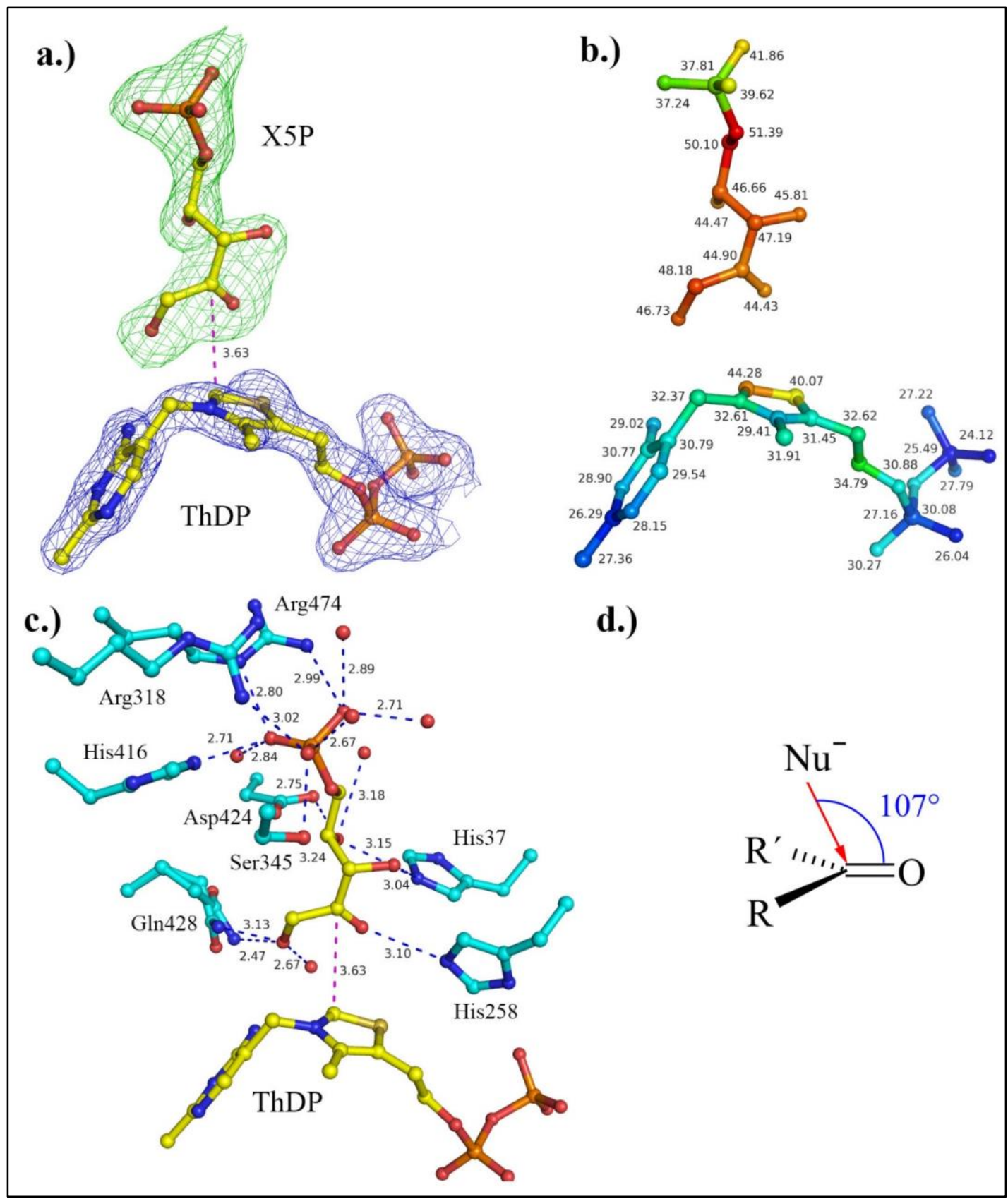

Fig 20: Michaelis complex of $\boldsymbol{h}$ TK-E366Q with donor substrate X5P. a.) X5P bound in close proximity to cofactor ThDP. Donor substrate X5P shown with a residue-omitted mFo-DFc map (contoured at $3 \sigma$ level, green) bound approx. $3.6 \AA$ away from the cofactor ThDP which is surrounded by a $2 \mathrm{mFo}-\mathrm{DF}$ c map (contoured at $1 \sigma$ level, blue). b.) Atoms of X5P and ThDP are colour-labelled according to their individual B-factors with most mobile atoms in red $\left(\approx 50 \AA^{2}\right)$ and less mobile ones in blue (24-26 $\AA^{2}$ ). c.) Interactions of X5P with the active site residues (shown in cyan) partially mediated by water molecules (shown as red spheres) are indicated by blue dashed lines. d.) optimal geometrical conditions for nucleophilic reactions (Burgi et al., 1973). 
Investigation of the amino acid residues responsible for the formation of the LBHB in $h \mathrm{TK}$ E366Q crystal structure in complex with X5P reveals different architecture to that of E160Q as well as the wild type (Fig 21). The electron density for the mutated amino acid residue (Gln366) is well defined and amide group is pointing to the N1' atom of the aminopyrimidine ring of ThDP with an inter-atomic distance of $2.80 \AA$. Unlike the hydroxyl function in the wild type, the amide is unable to promote the formation of the iminopyrimidine which is the tautomeric form of the aminopyrimidine to reversibly remove a proton from the $\mathrm{C} 2$ on the thiazolium ring. Thus, the "catalytic triad" of glutamate-iminopyrimidine-thiazol which is a paradigm in the ThDP-dependent enzymes has been interrupted, leading this protein mutant almost catalytically inactive. The length of the hydrogen bond between the oxygen atom on the carbonyl function from Gln366 and the oxygen atom on the hydroxyl function from Glu160 is approx. $2.59 \AA$. This hydrogen bond length is slightly longer than the typical LBHB (2.55 $\AA$ ) but much shorter than an ordinary hydrogen bond, leading to the assumption of the formation of a short ionic hydrogen bond. In addition, the single mutation from glutamate to glutamine changes the $\mathrm{p} K \mathrm{a}$ value of amino acid residue 366 which causes a $\mathrm{p} K \mathrm{a}$ gap between Glu160 and Gln366, thus the formation of a LBHB is not possible.

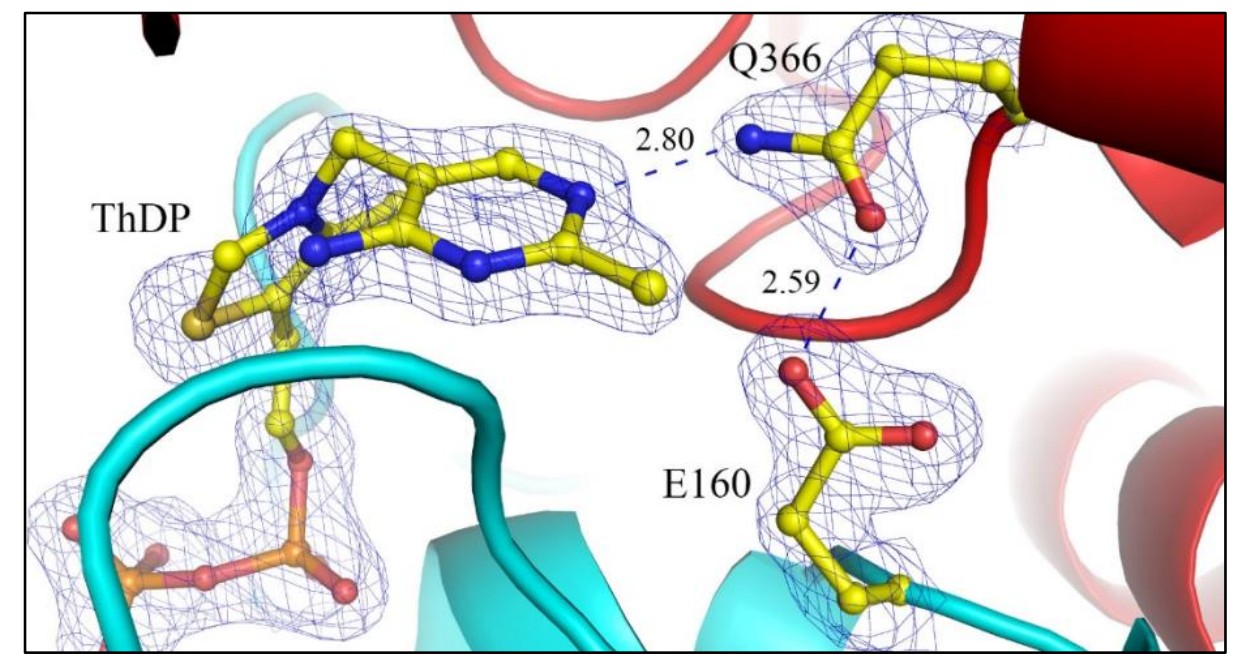

Fig 21: Active site architecture of $\boldsymbol{h T K}$ E366Q in complex with donor substrate X5P. Cofactor ThDP, residues Glu160 and Gln366 are shown in ball-stick representation surrounded by a $2 \mathrm{mFo}-\mathrm{DFc}$ map (contoured at $1 \sigma$ level, blue). The hydrogen interactions between N1' of ThDP and nitrogen atom of amide from Q366 as well as the oxygen atom of carbonyl from Q366 and the oxygen of hydroxyl from E160 are indicated by blue dashed line with inter-atomic distances. Two monomers forming the active site are shown in cartoon representation in red and cyan. 


\subsubsection{Steady-state kinetic analysis}

In order to investigate the function of the LBHB in the course of enzymatic catalysis, we have measured the enzymatic activity of $h \mathrm{TK}$ (wild type, E160Q, E160A, E165Q, and E366Q) for the conversion of $\mathrm{X} 5 \mathrm{P}$ and $\mathrm{R} 5 \mathrm{P}$ into $\mathrm{S} 7 \mathrm{P}$ and G3P. The measurement is based on a spectrophotometric steady-state assay which involves the participation of two auxiliary enzymes triose-phosphate isomerase and $s n$-glycerol-3-phosphate:NAD ${ }^{+}$2-oxidoreductase. The cleavage of X5P leads to the formation of glyceraldehyde 3-phosphate (GAP) which is further converted into glycerol 3-phosphate (G3P) by the two auxiliary enzymes and the concomitant oxidation of NADH is detected at $340 \mathrm{~nm}$. To compare the substrate affinity $(\mathrm{Km})$ for X5P in all studied proteins, the concentration of the acceptor substrate R5P was kept constant at $5 \mathrm{mM}$. Reaction progress curves (Fig 61) reveals that the reaction velocity is getting faster with increased donor substrate X5P concentration. The initial rate constants of the reaction were plotted against the utilized $\mathrm{X} 5 \mathrm{P}$ concentration and analysed according to the Michaelis-Menten and a substrate inhibition equation respectively. The results reveal that the data points are better explained by the substrate inhibition equation than the typical MichaelisMenten kinetics and the same holds true for the kinetics of active site mutants E160Q and E160A (Fig 61). This finding indicates that substrate inhibition which is known to occur with about $20 \%$ of all known enzymes is also involved within the reaction of transketolase. Typically, substrate inhibition of biological enzymes involves the binding of substrate to an extra, non-active site on the enzyme, which is not likely to happen on $h \mathrm{TK}$ as evidenced by the intensive structural studies. Therefore, the mechanism for the substrate inhibition to $h \mathrm{TK}$ needs further studies.

Human TK is purified as holo-enzyme with fully saturated cofactor and the activity is not dependent on the presence of excess ThDP and $\mathrm{Ca}^{2+}$ in the reaction mixture (Mitschke et al., 2010). This quasi-irreversible cofactor binding distinguishes human TK from E.coli and yeast transketolase which bind cofactor and divalent cation in a reversible manner (Asztalos et al., 2007; Wikner et al., 1997). However the cofactor binding for the active site variants might be influenced by the mutation, therefore extra ThDP and $\mathrm{Ca}^{2+}$ are supplemented to the assay mixture. The obtained steady-state kinetic constants for human TK wild type are very similar to the reported values described before. Mitschke and colleagues (Mitschke et al., 2010) reported a $k_{\text {cat }}$ value of $3.1 \pm 0.1 \mathrm{~s}^{-1}$ for the His-tagged protein and $6.3 \pm 0.1 \mathrm{~s}^{-1}$ for the His- tag removed protein at $30{ }^{\circ} \mathrm{C}$, which is comparable to the value of $2.79 \pm 0.06 \mathrm{~s}^{-1}$ that we obtained at $20{ }^{\circ} \mathrm{C}$. The E160Q variant shows an overall activity of $0.54 \pm 0.01 \mathrm{~s}^{-1}$ which is approx. 20 $\%$ of the wild type and the E160A variant exhibits around $8 \%$ of the overall activity of the wild type. The substrate affinity for these two mutants $(78.0 \pm 4.8 \mathrm{mM}$ for E160Q, $54.6 \pm 6.3$ 
$\mathrm{mM}$ for E160A) are very similar to that of the wild type $(73.9 \pm 6.7 \mathrm{mM})$, indicating that glutamate 160 in human TK is not involved in the substrate binding. This finding is further supported by the X-ray crystallography data that none of the substrates (X5P, F6P, S7P) have direct interactions with Glu160 (Lüdtke et al., 2013).
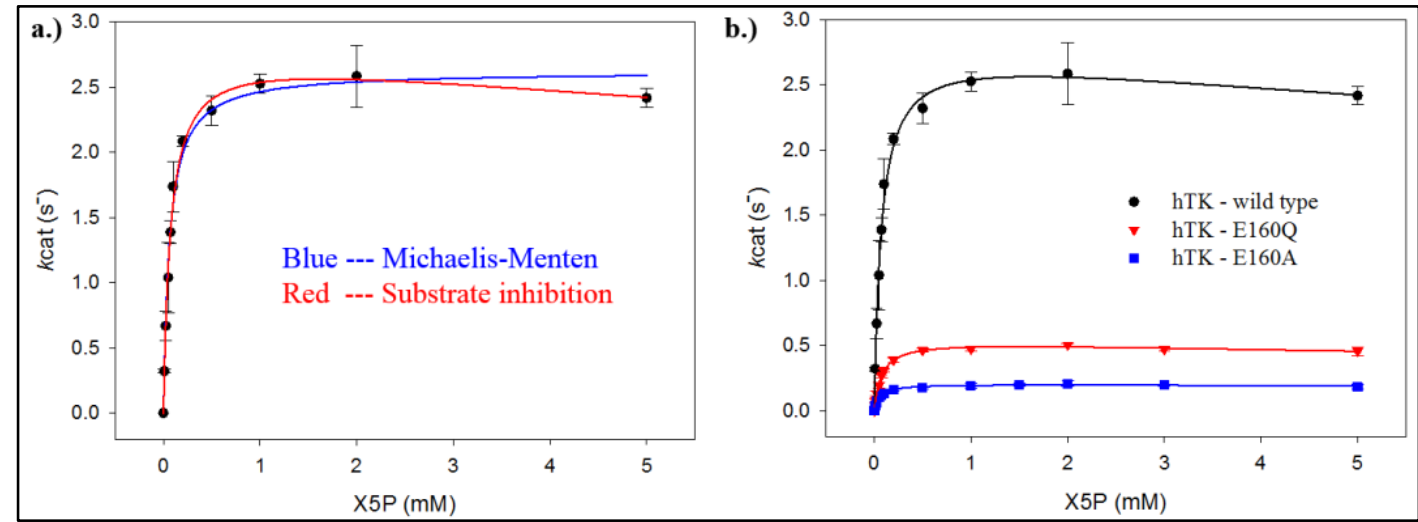

Fig 22: Steady-state kinetic analysis of $\boldsymbol{h}$ TK. The activity of $h$ TK wild type and LBHB-related active site variants were measured according to a two-enzyme coupled assay. a.) Kinetic plot of the steadystate activity for $h$ TK wild type. The dependency of the initial rates on the donor substrate X5P concentration was first analysed according to the Michaelis-Menten equation (blue) then according to a substrate inhibition equation (red). b.) Comparison of the steady-state activity of $h \mathrm{TK}$ wild type, E160Q and E160A. The activity of these three protein are plotted against the utilized substrate concentration with error bars showing the standard deviation from the mean values. The data points were respectively fitted according to the substrate inhibition equation.

The stability of human TK E165Q variant has dramatically decreased which makes crystallization and kinetic analysis very difficult. Luckily we managed to measure this variant by using low concentration of enzyme. This mutant shows an overall activity of $0.109 \pm 0.002$ $\mathrm{s}^{-1}$ which is only $4 \%$ of the wild type. Around $0.5 \%$ residual activity of $h$ TK E366Q was observed, which is consistent with the well-accepted mechanism that this conserved glutamate plays a pivotal role in promoting $\mathrm{C} 2-\mathrm{H}$ ionization and activation of thiamine diphosphate (Kluger and Tittmann, 2008). 
Table 4: Overview of the steady-state kinetic constants for $h$ TK wild type and LBHB-related active site variants. The constants for wild type, E160Q and E160A were obtained by fitting the data points according to the substrate inhibition equation whereas the constants for E165Q and E366Q were obtained by fitting the data points according to the Michaelis-Menten equation. $K_{\mathrm{I}}$ represents the apparent inhibition equilibrium constant of the putative substrate inhibition. The standard deviation from the mean values of triplicates are given.

\begin{tabular}{ccccc}
\hline Protein & $\boldsymbol{k}_{\text {cat }}\left(\mathbf{s}^{-1}\right)$ & $\boldsymbol{K}_{\mathbf{m}}(\mathbf{X 5 P}, \boldsymbol{\mu M})$ & $\boldsymbol{k}_{\text {cat }} / \boldsymbol{K}_{\mathbf{m}}\left(\mathbf{s}^{-1} \mathbf{M}^{-1}\right)$ & $\boldsymbol{K}_{\mathbf{I}}(\boldsymbol{\mu M})$ \\
\hline$h$ TK - wt & $2.79 \pm 0.06$ & $73.9 \pm 6.7$ & 37754 & $35.9 \pm 14.6$ \\
$h$ TK - E160Q & $0.54 \pm 0.01$ & $78.0 \pm 4.8$ & 6923 & $29.4 \pm 6.9$ \\
$h$ TK - E160A & $0.21 \pm 0.02$ & $54.6 \pm 6.3$ & 3846 & $70.8 \pm 62.3$ \\
$h$ TK - E165Q & $0.109 \pm 0.002$ & $149.8 \pm 10.4$ & 728 & ND \\
$h$ TK - E366Q & $0.0125 \pm 0.0014$ & $16269 \pm 2670$ & $7.7 \mathrm{e}^{-7}$ & ND \\
\hline
\end{tabular}

In addition, a possible lag phase could be monitored for the E160Q variant in the first $25 \mathrm{~s}$ with low donor substrate concentration in the reaction process, which is absent in the reaction of higher $\mathrm{X} 5 \mathrm{P}$ concentration. One explanation for the lag phase would be that the communication between the two actives sites is interrupted by the single mutation thus binding of X5P to the first active site couldn't inhibit the binding to the second "dormant" site, leading to formation of the lag phase.

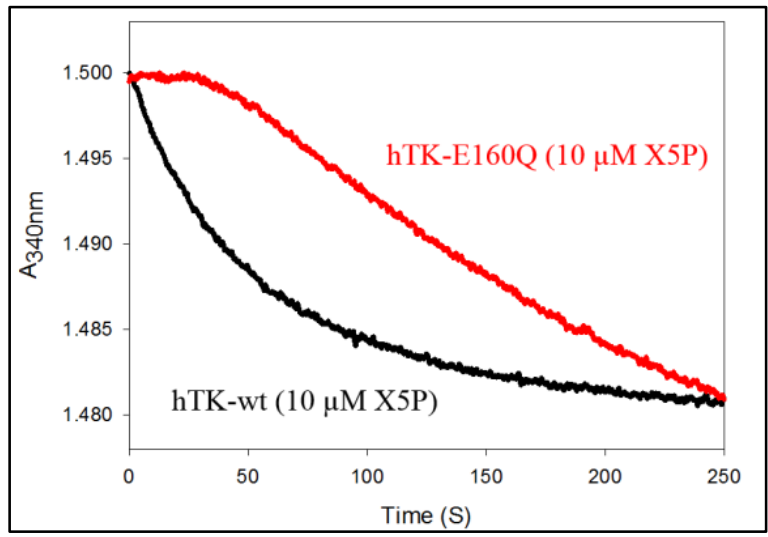

Fig 23: Possible lag phase in the steadystate assay observed on $h$ TK-E160Q. Progress curves for $h$ TK-wild type (black) and E160Q (red) with low $(10 \mu \mathrm{M})$ donor substrate X5P concentration. Please note that a possible lag phase within the first $25 \mathrm{~s}$ of the reaction could be observed with $h$ TK-E160Q which is absent on wild type.

\subsubsection{Pre-steady-state kinetic analysis by stopped-flow spectroscopy}

In order to analyse the function of the LBHB for the donor half reaction of transketolase catalysis, single-mixing stopped-flow spectroscopy was employed to monitor the depletion of 
AP at $325 \mathrm{~nm}$ and the formation of IP at $295 \mathrm{~nm}$ when human TK is rapidly mixed with the donor substrate F6P. The reaction transients measured at $325 \mathrm{~nm}$ for the E160Q and E160A variants revealed a biphasic process while a single phase was observed for the wild type (Fig 24). The second phase ambiguously represents the depletion of the AP form when substrateThDP intermediate is accumulated on the enzyme, but the chemical origin of the first phase is not very clear yet. Plotting of the $k_{\mathrm{obs}}$ of the first phase at $325 \mathrm{~nm}$ against the utilized F6P concentration revealed a hyperbolic dependence for both E160Q and E160A variants. Maximal $k_{\text {obs }}$ of $103.61 \pm 5.66 \mathrm{~s}^{-}, K_{\mathrm{s}}^{\text {app }}$ of $2.94 \pm 0.40 \mathrm{mM}$ and maximal $k_{\text {obs }}$ of $100.13 \pm 4.81$ $\mathrm{s}^{-}$and $K_{\mathrm{s}}{ }^{\text {app }}$ of $0.39 \pm 0.09 \mathrm{mM}$ were obtained for the two variants respectively (Fig 25). Kinetic parameters of the fast pre-equilibrium phase for the IP form measured at $295 \mathrm{~nm}$ could not been obtained due to the scattered data points.
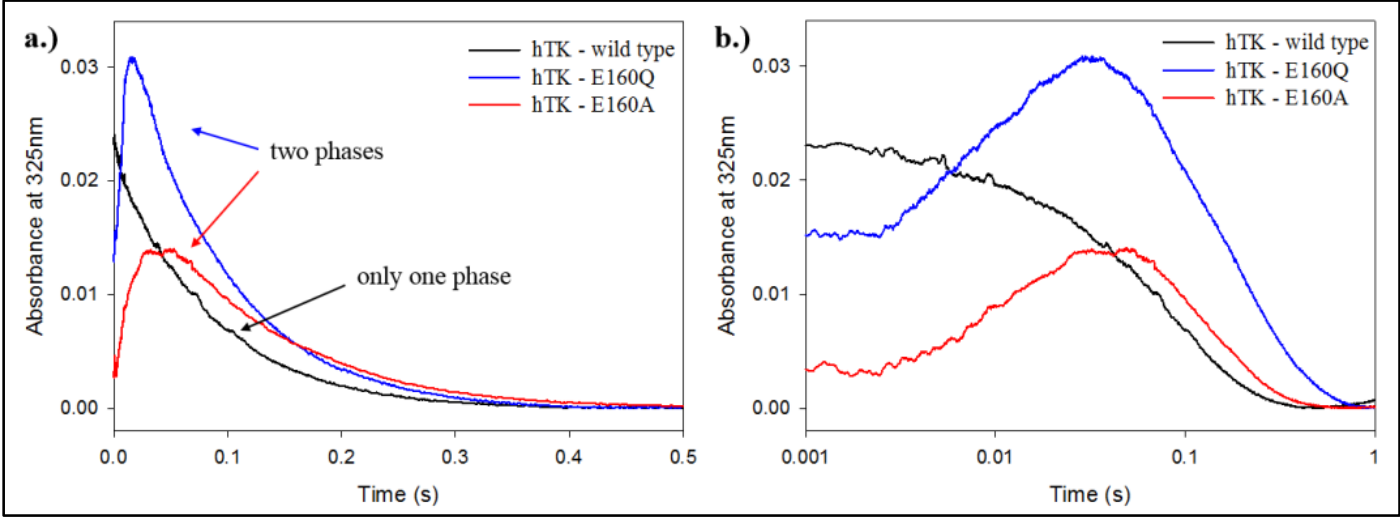

Fig 24: Representative progress curves of the pre-steady-state kinetic analysis of $h$ TK and LBHBrelated active site variants. Reaction conditions refer to Fig 26. Saturation donor substrate F6P (10 $\mathrm{mM}$ ) were used for these reaction transients. a.) Normal scale representation for the reaction progress curves of the donor half reaction of $h$ TK wild type, E160Q and E160A mixed with substrate F6P (10 $\mathrm{mM}$ ) measured at $325 \mathrm{~nm}$ at $4{ }^{\circ} \mathrm{C}$. Please note that the two variants undergo a two-phase reaction while only one phase was observed on the wild type. b.) Logarithmic scale representation for the same reaction transients. 


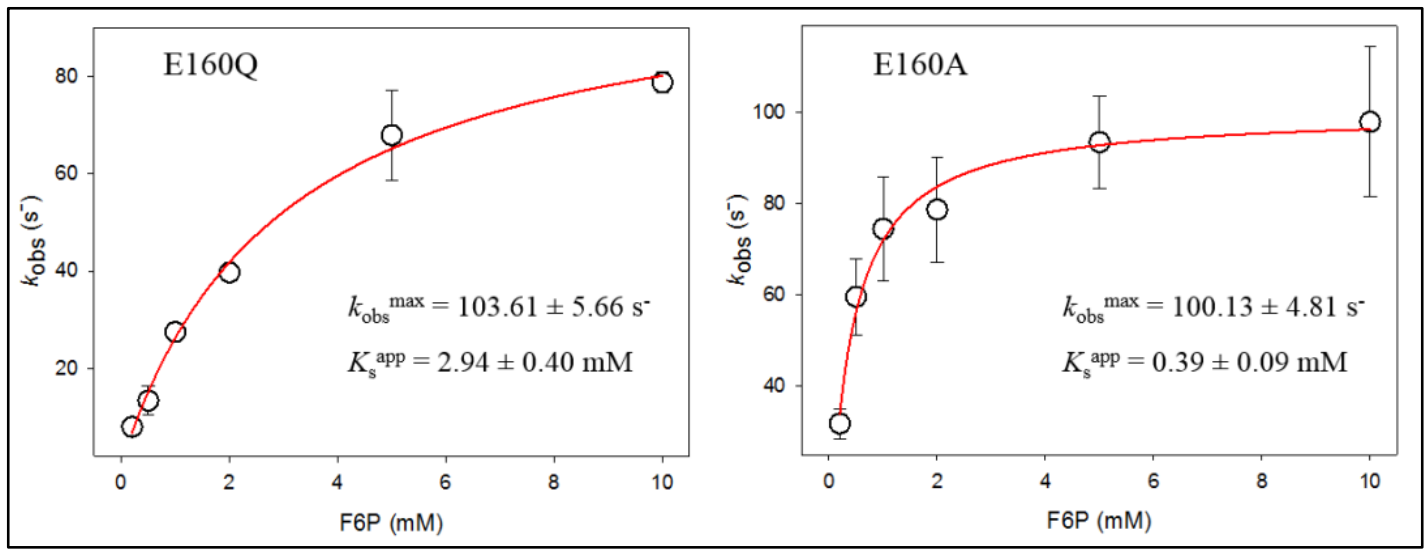

Fig 25: The first phase of the pre-steady-state kinetics for human TK E160Q and E160A. The rate constants of the first fast phase of the donor half reaction for $h$ TK E160Q (left) and E160A (right) measured at $325 \mathrm{~nm}$ are plotted against the utilized F6P concentrations. Hyperbolic dependency is observed and the $k_{\mathrm{obs}}{ }^{\max }$ and $K_{\mathrm{s}}{ }^{\text {app }}$ are indicated for both variants.

$$
k_{\mathrm{obs}}=k_{-2}+\frac{k_{\mathrm{obs}}^{\max *[\mathrm{~F} 6 \mathrm{P}]}}{K_{\mathrm{s}}^{\mathrm{app}}+[\mathrm{F} 6 \mathrm{P}]}
$$

Equation 3: hyperbolic equation used for the analysis of the donor half reaction of $h \mathrm{TK}$ with substrate F6P. $K_{\mathrm{s}}{ }^{\text {app }}=$ the dissociation constant of the pre-equilibrium. $k_{-2}=$ the rate constant of the carbonyl elimination. $k_{\mathrm{obs}}{ }^{\max }=$ first order rate constant at saturation substrate concentration.

$$
k_{\mathrm{obs}}=k_{-2}+\frac{k_{\mathrm{obs}}^{\max *[\mathrm{~F} 6 \mathrm{P}]^{\mathrm{n}}}}{\left(K_{\mathrm{s}}^{\mathrm{app}}\right)^{\mathrm{n}}+[\mathrm{F} 6 \mathrm{P}]^{\mathrm{n}}}
$$

Equation 4: Hill equation used for the analysis of the donor half reaction of $h$ TK with substrate F6P. $K_{\mathrm{s}}^{\mathrm{app}}=$ the dissociation constant of the pre-equilibrium. $k_{-2}=$ the rate constant of the carbonyl elimination. $k_{\mathrm{obs}}{ }_{\max }=$ first order rate constant at saturation substrate concentration. $\mathrm{n}=$ Hill coefficient, when $\mathrm{n}<1$, there is negative cooperativity, when $\mathrm{n}=1$, there is no cooperativity, when $\mathrm{n}>1$, there is positive cooperativity.

Plotting of the rate constant for the second phase against the utilized F6P concentration are shown in Fig 26. The data points are better fitted according to the Hill equation (equation 4) than the normal hyperbolic equation (equation 3). The LBHB containing wild type exhibits a 2-fold velocity increase relative to the E160Q variant in which the LBHB is replaced by an ordinary hydrogen bond. A decrease of substrate affinity is also observed on the E160Q mutant (wild type, $0.66 \pm 0.06 \mathrm{mM}$; E160Q, $0.94 \pm 0.07 \mathrm{mM}$ ). The dissociation of the covalent F6PThDP intermediate $\left(k_{-2}\right)$ has drops 5-fold in the variant as well (Table 5). The most important observation lies on the cooperativity for the donor substrate F6P binding. The Hill coefficient 
of wild type (1.56 \pm 0.26 for AP depletion, $1.49 \pm 0.43$ for IP formation) strongly indicates positive cooperativity while no cooperativity could be detected for the E160Q variant. The reaction constant for the E160A mutant lies between wild type and E160Q and the substrate affinity is similar to the wild type, suggesting that the binding for the donor half reaction is not affected by the abolishment of residue Glu160. Although structural data is not available for the E160A variant, we could deduce that a water molecule probably replaces the glutamate at the same position and acts as an acid-base catalyst. The crystal structure of the same variant of E.coli TK has been determined and a water molecule is observed at this position (data not published).
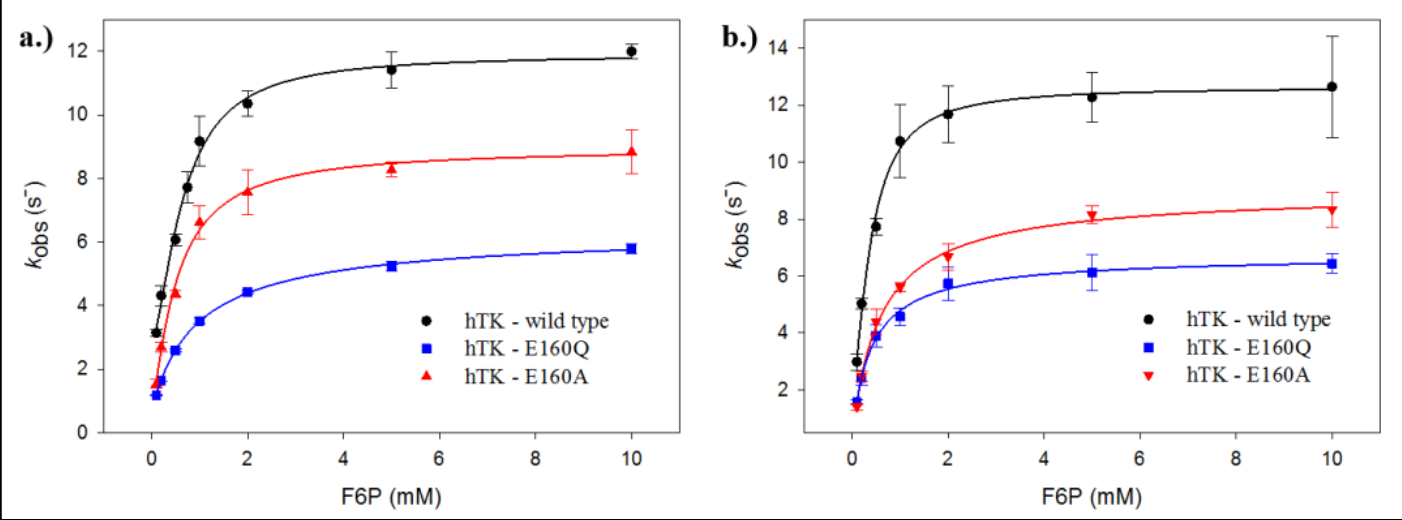

Fig 26: Pre-steady-state kinetic analysis of $\boldsymbol{h T K}$. The donor half reaction (also named pre-steadystate) of $h \mathrm{TK}$ wild type and LBHB-related active site variants were measured by single-mixing stoppedflow spectroscopy. Typical reactions were performed by mixing $4 \mathrm{mg} / \mathrm{ml} h \mathrm{TK}$ with increasing concentration of fructose 6-phosphate (F6P) in $5 \mathrm{mM} \mathrm{CaCl}_{2}, 50 \mathrm{mM}$ glycylglycine (pH 7.6) and measured by UV-Vis spectrometer at specific wavelength at $4{ }^{\circ} \mathrm{C}$. Reaction constants were determined according to a single exponential equation $\left(\mathrm{A}=\mathrm{A} 1 \mathrm{e}^{-k^{*} t}+\right.$ offset $)$. a.) Rate constants for the depletion of AP form measured at $325 \mathrm{~nm}$ were plotted against the utilized F6P concentration and the data points were analysed according to a Hill equation. b.) Rate constants for the formation of IP form measured at $295 \mathrm{~nm}$ were plotted against the utilized F6P concentration and the data points were analysed according to a Hill equation.

The formation the IP form measured at $295 \mathrm{~nm}$ revealed identical kinetic features thus reflects a same chemical process that happened at both wavelengths. Again, a biphasic reaction transient was observed for all three measured proteins. The values of the maximal $k_{\text {obs }}$ for all three proteins are slightly higher than that of the depletion of AP form, which could be partially explained by the knowledge that aromatic amino acids such as Tyr, Trp and Phe also have absorbance at this wavelength. 
Table 5: Overview of the pre-steady-state kinetic constants for $h$ TK wild type and LBHB-related active site variants. The constants for wild type, E160Q and E160A were obtained by fitting the data points according to the Hill equation as explained before. $k_{-2}=$ rate constant of the reverse reaction for the formation of F6P-ThDP covalent intermediate; $k_{\mathrm{obs}}{ }^{\max }=$ first order rate constant at saturation substrate concentration; $K_{\mathrm{S}}^{\mathrm{app}}=$ apparent equilibrium constant for the formation of F6P-ThDP covalent intermediate; The standard deviation from the mean values of triplicates are given.

\section{n}

\begin{tabular}{|c|c|c|c|c|c|}
\hline & Protein & $k_{-2}\left(\mathrm{~s}^{-1}\right)$ & $k_{\text {obs }}{ }^{\max }\left(s^{-1}\right)$ & $K_{\mathrm{s}}^{\mathrm{app}}(\mathbf{m M})$ & $\begin{array}{c}n \\
\text { (Hill coefficient) }\end{array}$ \\
\hline \multirow{3}{*}{$\begin{array}{l}\text { AP form } \\
\text { depletion }\end{array}$} & $h \mathrm{TK}-\mathrm{wt}$ & $2.84 \pm 0.53$ & $9.06 \pm 0.72$ & $0.66 \pm 0.06$ & $1.56 \pm 0.26$ \\
\hline & $h \mathrm{TK}-\mathrm{E} 160 \mathrm{Q}$ & $0.47 \pm 0.24$ & $5.91 \pm 0.43$ & $0.94 \pm 0.07$ & $0.90 \pm 0.10$ \\
\hline & $h \mathrm{TK}-\mathrm{E} 160 \mathrm{~A}$ & $0.62 \pm 0.49$ & $8.32 \pm 1.21$ & $0.52 \pm 0.12$ & $1.23 \pm 0.30$ \\
\hline \multirow{3}{*}{$\begin{array}{l}\text { IP form } \\
\text { formation }\end{array}$} & $h \mathrm{TK}-\mathrm{wt}$ & $1.85 \pm 1.50$ & $10.78 \pm 1.74$ & $0.40 \pm 0.10$ & $1.49 \pm 0.43$ \\
\hline & $h \mathrm{TK}-\mathrm{E} 160 \mathrm{Q}$ & $0.05 \pm 1.62$ & $6.71 \pm 1.95$ & $0.39 \pm 0.20$ & $0.91 \pm 0.33$ \\
\hline & $h \mathrm{TK}-\mathrm{E} 160 \mathrm{~A}$ & ND & $9.01 \pm 0.30$ & $0.57 \pm 0.06$ & $0.93 \pm 0.07$ \\
\hline
\end{tabular}

\subsubsection{Circular Dichroism (CD) analysis of the tautomeric and protonic states of ThDP}

One of the criteria for forming LBHBs is that the donor and acceptor of the hydrogen bond must have identical $\mathrm{pKa}$ values so that the hydrogen could be equally shared between them (Cleland and Kreevoy, 1994). In order to investigate the impact of the LBHB on the pKa values, a $\mathrm{pH}$ titration experiment was performed based on a method described earlier (Lüdtke et al., 2013; Meyer et al., 2010) and protein samples in a series of $\mathrm{pH}$ values were measured by Circular Dichroism (CD) spectrometer. According to literatures, the six-membered aminopyrimidine ring of enzyme-bound ThDP adopts three protonic and tautomeric states under physiological conditions. Each of them has an individual spectral characteristic in the CD spectroscopy. The AP form exhibits a negative CD signal at 320-330 nm while the IP tautomer gives rise to a positive CD signal centered at around 300-310 nm (Nemeria et al., 2007). The $\mathrm{CD}$ signal of the conjugate acid $\mathrm{APH}^{+}$form has not been observed for a long time, but former colleagues in our group have identified a characteristic negative $\mathrm{CD}$ signal on ZmPDC and XFPK at $290 \mathrm{~nm}$ which decreases with increasing pH value (Schneider, 2014; Wechsler, 2014). This assignment seems to be correct, but re-evaluation of the CD spectra of a ThDP titration experiment on E.coli TK E411A (Asztalos, 2008) would rule out this assumption. The appearance of negative band at $290 \mathrm{~nm}$ was observed which is impossible to 
be the $\mathrm{APH}^{+}$form due to the fact that $\mathrm{APH}^{+}$can't be formed on this variant. Therefore, the negative CD band at $290 \mathrm{~nm}$ is assigned as the AP form with protonated glutamate and the negative CD band at 320-330 nm would be the AP form with deprotonated glutamate. In this way, the chemical origin for these bands are more reasonably explained.

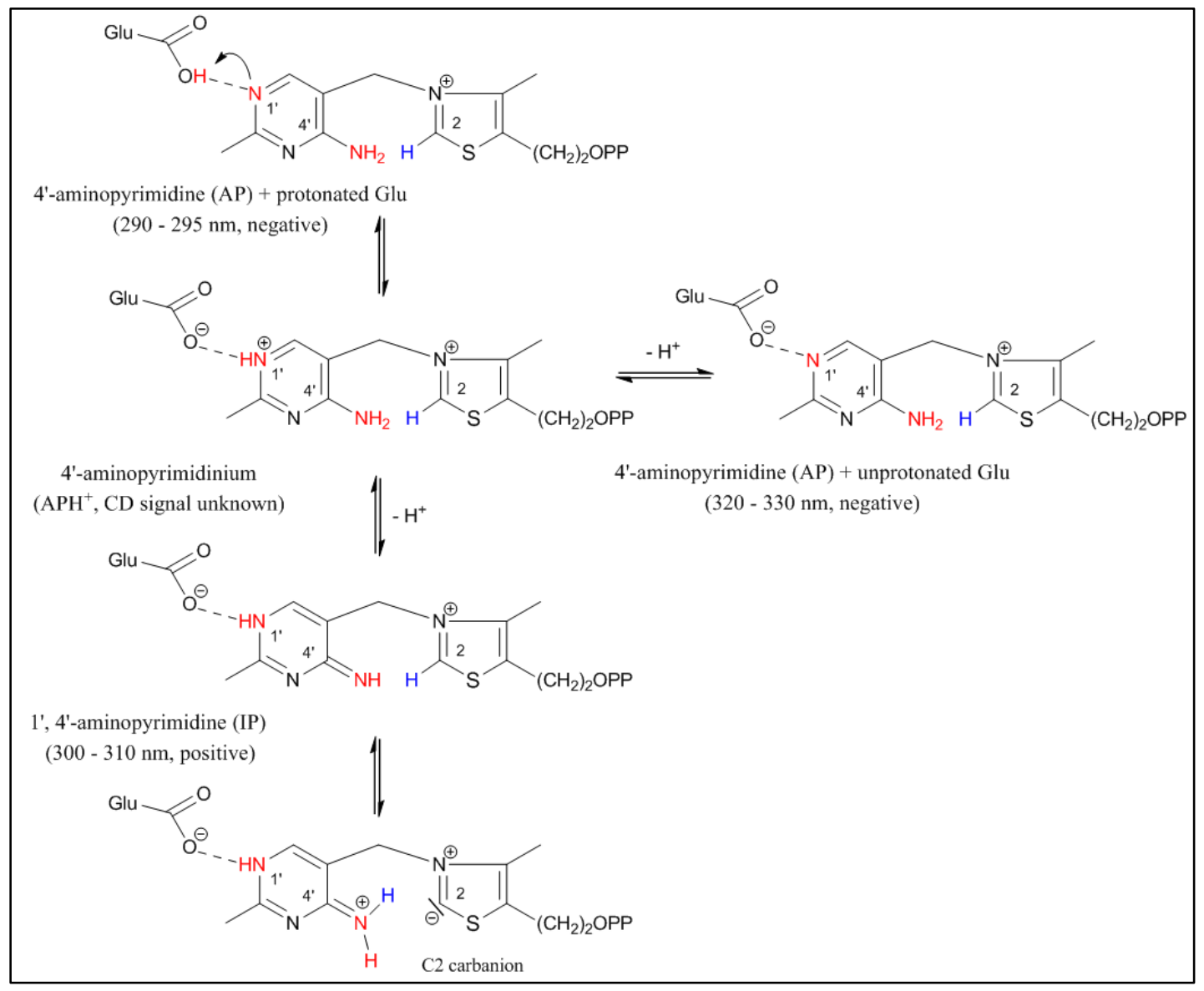

Fig 27: Protonic and tautomeric forms of ThDP and Glu366 in the process of C2-H ionization.

The three tautomeric states of the six-membered aminopyrimidine ring in combination with the protonic states of Glu366 are indicated. Selected atoms are labelled and the Circular Dichroism (CD) spectral features are included. Please note that the negative signature at 290-295 $\mathrm{nm}$ of the aminopyrimidine (AP) form with protonated Glu is observed for the first time.

We have performed $\mathrm{pH}$ titration experiments for human TK wild type and several LBHBrelated active site variants, the obtained CD spectra reveals different features for these studied proteins. For the wild type enzyme, the characteristic negative band centered at $325 \mathrm{~nm}$ for the AP tautomer with deprotonated glutamate increases with the $\mathrm{pH}$ value and reaches a maximal amplitude at $\mathrm{pH}$ 7.5. The same holds true for the IP tautomeric form which exhibits a positive $\mathrm{CD}$ at around $300 \mathrm{~nm}$. An interesting observation lies at the wavelength of $290 \mathrm{~nm}$ where a 
negative band overlaps with the positive IP band and it decreases with increasing $\mathrm{pH}$ value. Those features are quite similar to the AP form with protonated glutamate described earlier therefore could be assigned as AP form with the protonated glutamate. By plotting the amplitude of AP form against the corresponding $\mathrm{pH}$ and analysing the data points according to a single proton titrating equation (equation 5), a pKa value of $5.96 \pm 0.07$ for the ([AP] + $[\mathrm{IP}]) /\left[\mathrm{APH}^{+}\right]$equilibrium was calculated which is very close to the $\mathrm{p} K \mathrm{a}$ value reported earlier using similar method but starting at pH 6.0 (Lüdtke et al., 2013).

$$
\begin{aligned}
& \text { a.) } \mathrm{A}=\frac{\mathrm{A}_{\max }}{1+10^{(\mathrm{p} K \mathrm{a}-\mathrm{pH})}}+\mathrm{A}_{0} \\
& \text { b.) } \mathrm{A}=\frac{\mathrm{A}_{\max }}{1+10^{(\mathrm{pK} \mathrm{a} 1-\mathrm{pH})}+10^{(\mathrm{p} K \mathrm{a} 2-\mathrm{pH})}}+\mathrm{A}_{0}
\end{aligned}
$$

Equation 5: Single (a) and double (b) proton titration equation.

Dramatically different CD spectra were recorded for the E160Q variant in which the LBHB is replaced by an ordinary hydrogen bond. The characteristic AP band with deprotonated glutamate is absent at lower $\mathrm{pH}$ value $(<6.0)$ and starts to appear from $\mathrm{pH}$ 6.0. The AP form with protonated glutamate which could only be observed at low $\mathrm{pH}(<5.0)$ on wild type accumulates abundantly on E160Q variant at larger $\mathrm{pH}$ range $(5.0-8.0)$ and it continues decreasing when the $\mathrm{pH}$ value increases. By analysing the $\mathrm{pH}$ dependency of the AP form according to equation 5 , a $\mathrm{pKa}$ value of $7.23 \pm 0.06$ was obtained which is around $1.5 \mathrm{pH}$ units higher than that of the wild type.

Closer inspection on the amplitude of the AP tautomer at $325 \mathrm{~nm}$ reveals that the E160Q variant only exhibits half of the intensity of the wild type, reflecting chemically nonequivalence of the two corresponding active sites of $h \mathrm{TK}$ E160Q variant. The explanation for this behaviour is that the communication between the two active sites of human TK has been interrupted by the Glu $\rightarrow$ Gln mutation thus causing different behaviour in individual active site. The $\mathrm{pH}$ dependency of the AP form on E160A variant (Fig 28) revealed a two-step process which leads to the formation of two individual $\mathrm{pKa}$ values. Analysis of the $\mathrm{pH}$ dependency of the IP and $\mathrm{APH}^{+}$form at 300 and $290 \mathrm{~nm}$ lead to the same conclusion. Interestingly, the $\mathrm{pKa}_{1}$ $(7.76 \pm 0.23)$ and $\mathrm{pKa} 2(6.13 \pm 0.13)$ are very close to the $\mathrm{pKa}$ values of E160Q $(7.23 \pm 0.06)$ and wild type (5.96 \pm 0.07$)$, suggesting that the E160A variant exhibits features partially from wild type ( $\mathrm{pKa}_{2}$ and full amplitude of AP with deprotonated glutamate) and partially from E160Q ( $\mathrm{pKa} \mathrm{a}_{1}$ and full amplitude of AP with protonated glutamate). 
Glu366 in human TK is the conserved glutamate residue in ThDP-dependent enzymes which acts as the general acid-base catalyst to activate the cofactor though the catalytic triad glutamate-imimopyrimidine-thiazol (Shaanan and Chipman, 2009). Mutation of this glutamate to glutamine causes the absence of IP and AP with protonated glutamate tautomer on the enzyme and only AP form with deprotonated glutamate could be detected by CD. Careful analysis revealed that the relative amplitude of the AP form with protonated glutamate (glutamine in this variant) remains unaffected, which is consistent with the general accepted proposal that replacement of this conserved glutamate by catalytically inactive glutamine disables the interconversion of the tautomeric forms of AP, thus rendering this protein mutant almost dead (Kluger and Tittmann, 2008).
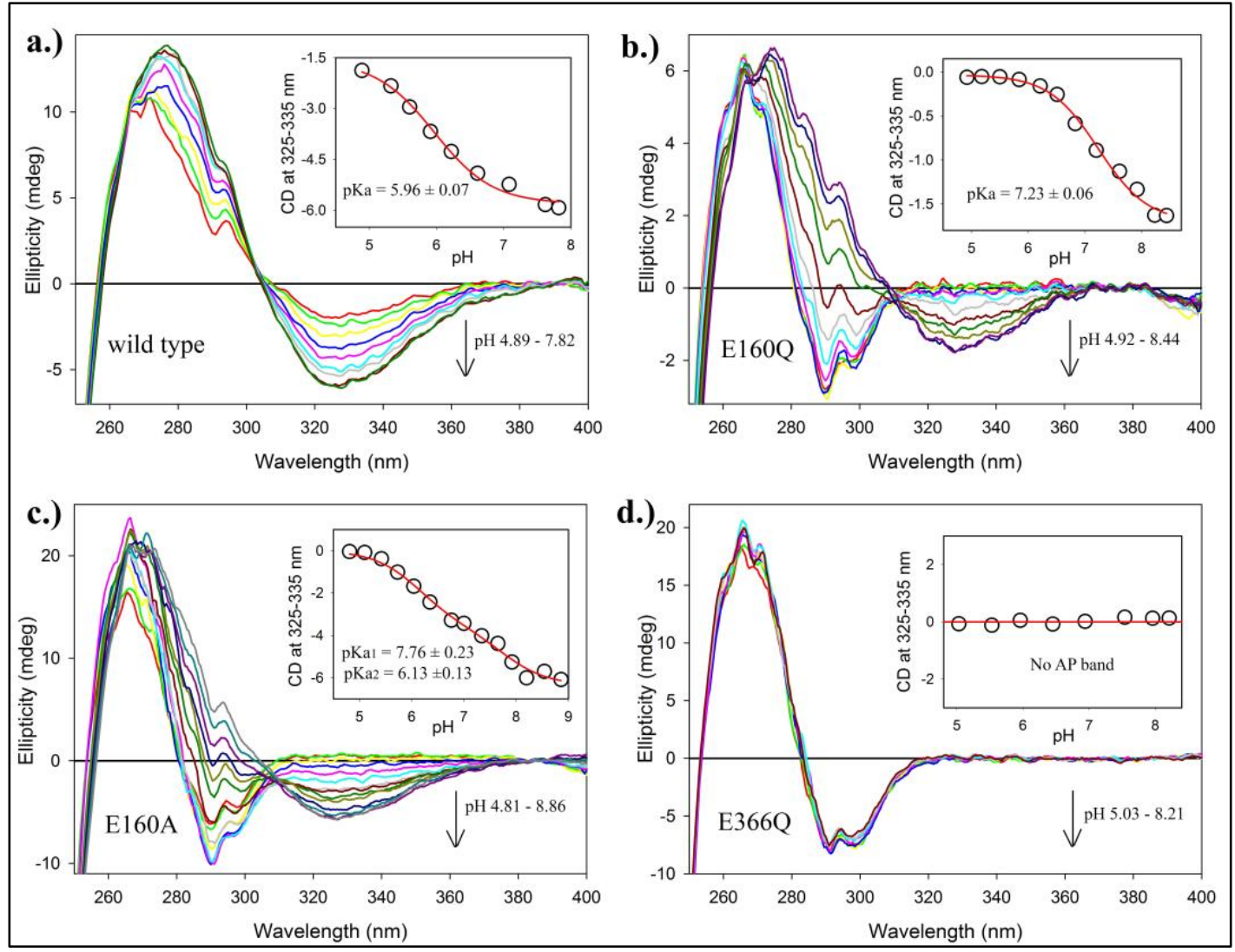

Fig 28: Near-UV CD spectra of the tautomeric and protonic states of ThDP at different pH values

for human TK wild type and LBHB-related active site variants. Samples containing $1.5 \mathrm{mg} / \mathrm{ml}$ enzyme in $20 \mathrm{mM}$ MES, $20 \mathrm{mM}$ glycylglycine were titrated by addition of Tris base (1M, pH 11) and the $\mathrm{pH}$ values were measured using a Mini trode $\mathrm{pH}$ electrode (Hamilton). The measurements were performed at $20^{\circ} \mathrm{C}$ with a path length of $10 \mathrm{~mm}$ and recorded between $250-400 \mathrm{~nm}$. a.) human TK wild type. Inset: $\mathrm{pH}$ dependence of AP formation at 325-335 nm (average value). The $\mathrm{pKa}$ was determined using eq 5.a. b.) E160Q. c.) E160A. The pKa was determined using eq 5.b. d.) E366Q. 
As mentioned before, the stability of human TK E165Q variant has dramatically decreased which sets huge obstacle for the $\mathrm{pH}$ titration experiment. In order to analyse the $\mathrm{pH}$ dependency of the three tautomeric forms on E165Q variant, the same $\mathrm{pH}$ titration experiment was conducted with the protein kept on ice and measured at lower temperature $\left(10^{\circ} \mathrm{C}\right)$. The results suggested a dramatic different behaviour of the ([AP] + [IP] $) /\left[\mathrm{APH}^{+}\right]$equilibrium on this variant. At acidic pH 5.72, only a small fraction of AP with deprotonated glutamate (at 320-330 nm) could be observed and nearly no IP form (at 300-310 nm) and no AP with protonated glutamate (at 290-295 nm) existing, which indicates the predominant accumulation of the $\mathrm{APH}^{+}$form based on the current knowledge that no other forms are existing. As $\mathrm{pH}$ goes up, the $\mathrm{APH}^{+}$form would be converted into the AP form with protonated glutamate indicated by the increasing negative band at 290-295 $\mathrm{nm}$. This increasing tendency with $\mathrm{pH}$ seems to be completely inverted compared with wild type and E160Q/A variants. However, the negative band at 290-295 nm was not saturated even at the highest measured $\mathrm{pH}(8.31)$, so it might continue increasing until the maximal amplitude reaches and then decrease at even higher $\mathrm{pH}$ range. Therefore, $\mathrm{pH}$ titration experiment for this variant starting from basic $\mathrm{pH}$ (for example, $\mathrm{pH}$ 8.0) should be performed in the future.

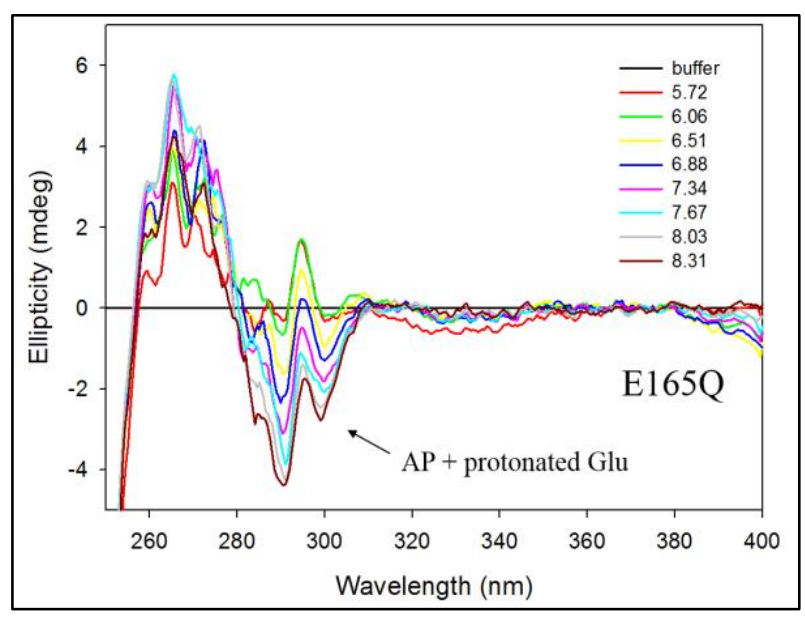

Fig 29: Near-UV CD spectra of the tautomeric and protonic states of ThDP at different $\mathrm{pH}$ values for human TK E165Q. Protein $(1.5 \mathrm{mg} / \mathrm{ml})$ in $20 \mathrm{mM}$ MES, $20 \mathrm{mM}$ glycylglycine was titrated by addition of Tris base (1M, pH 11) on ice and the $\mathrm{pH}$ values were measured using a Minitrode $\mathrm{pH}$ electrode (Hamilton). The measurements were performed at $10{ }^{\circ} \mathrm{C}$ with a path length of $10 \mathrm{~mm}$ and recorded between $250-400 \mathrm{~nm}$.

\subsubsection{Temperature jump ( $T$-jump) analysis of the tautomeric and protonic states of ThDP}

According to the Van't Hoff's early extraordinary work in the last century, the equilibrium constants of all reactions vary with temperature. Temperature-jump technique is a recently developed technique which enables the detection of fast reactions with half-lives of only a few microseconds (Gutfreund, 1981) . At the initial temperature, the reaction volume is in 
equilibrium and then rapidly perturbed by a fast change in temperature which is generated by a short pulse of electrical discharge at high voltage (up to $12 \mathrm{kV}$ ) that causes a temperature rise of around $10{ }^{\circ} \mathrm{C}$ within a few microseconds (dead time of T-jump). The reaction system therefore relaxes and proceeds until a new equilibrium for the higher temperature reaches.

In order to investigate the temperature dependency for the $([\mathrm{AP}]+[\mathrm{IP}]) /\left[\mathrm{APH}^{+}\right]$equilibrium, temperature jump experiments were performed on human TK with a temperature rise from 12 ${ }^{\circ} \mathrm{C}$ to $20-21{ }^{\circ} \mathrm{C}$. AP and IP tautomer were measured under this condition by an UV-Vis spectrometer at their corresponding wavelengths ( $325 \mathrm{~nm}$ for AP, $294 \mathrm{~nm}$ for IP). The progress curve at $325 \mathrm{~nm}$ reveals that the AP form undergoes a depletion with a rate constant of 11559 $\pm 408 \mathrm{~s}^{-}$when temperature rises up. The small amplitude around $0.003 \mathrm{AU}$ which is an averaged values of 50 measurements indicates that the chemical equilibrium only undergoes a minor change with temperature. A two-step process was observed at $294 \mathrm{~nm}$ which is the representative wavelength for the IP tautomer. The first fast step with a rate constant of 13950 $\pm 3417 \mathrm{~s}^{-}$represents a depletion of the IP form which is as fast as the AP depletion.

Trials at higher starting temperatures lead to protein aggregation, reflecting the poor thermal stability of human transketolase which has already been revealed previously (Lüdtke, 2012). The same experiments performed on E160Q variant was unsuccessful due to the same reason even under the same condition for the wild type. Each progress curve remains flat while the baseline rises with time due to minor protein aggregation (Fig 60). The average of 50 measurements at $325 \mathrm{~nm}$ turned out to be flat, which reflects the interruption of the proton wire by the mutation and hampers the interconversion of the three tautomeric and protonic states of ThDP.
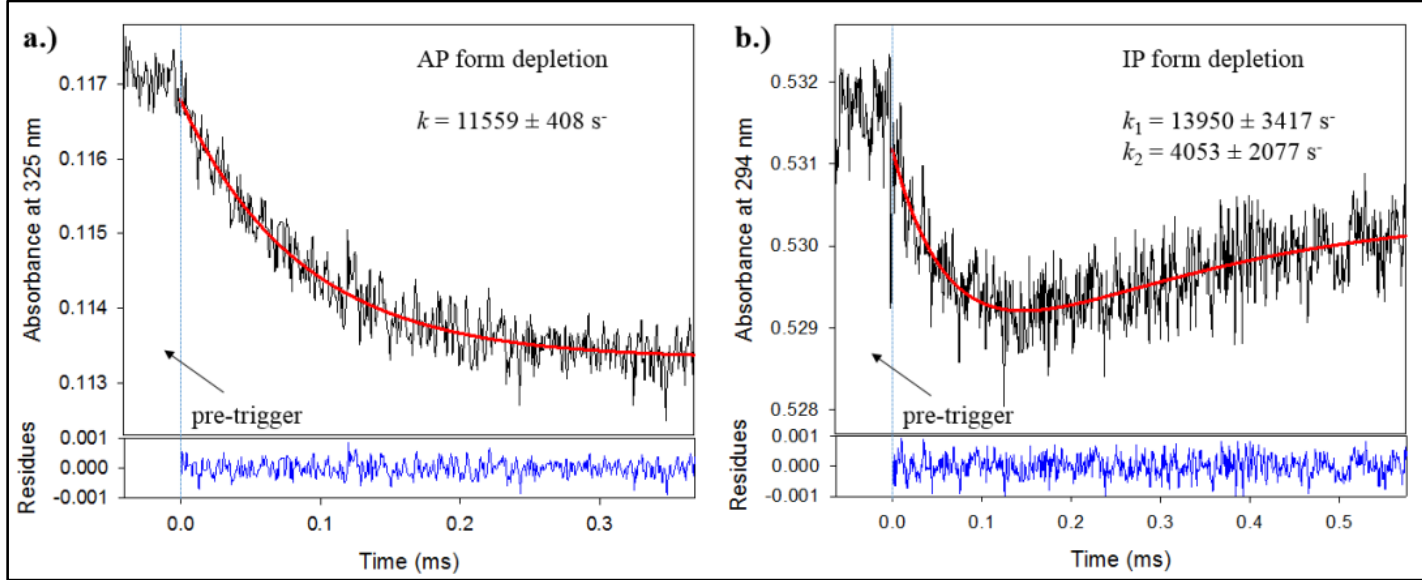

Fig 30: Temperature jump analysis of the tautomeric and protonic forms of ThDP. The two tautomeric forms (namely AP and IP) of ThDP on $h \mathrm{TK}\left(5 \mathrm{mg} / \mathrm{ml}\right.$ in $50 \mathrm{mM}$ glycylglycine, $5 \mathrm{mM} \mathrm{CaCl}_{2}$, 
$\mathrm{pH}$ 7.6) were studied by using the temperature jump relaxation technique with a jump of $10{ }^{\circ} \mathrm{C}$ (12 to $22{ }^{\circ} \mathrm{C}$ ). The obtained reaction progress curves are the average of 50 traces due to small amplitude and high signal-to-noise ratio. The area before the reaction starting point is the pre-trigger stage. a.) The depletion of the AP form measured at $325 \mathrm{~nm}$. Rate constant of AP depletion was calculated by fitting the curve according to a single exponential equation (red, $\mathrm{A}=\mathrm{A} 1 \mathrm{e}^{-\mathrm{k}^{*} \mathrm{t}}+$ offset). b.) The depletion of the IP form measured at $294 \mathrm{~nm}$. Rate constants of IP depletion and a second phase were calculated by fitting the curve according to a double exponential equation (red, $\mathrm{A}=\mathrm{A} 1 * \mathrm{e}^{-k 1 * \mathrm{t}}+\mathrm{A} 2 * \mathrm{e}^{-k 2 * t}+$ offset). Residues for both fittings are shown below the reaction transient.

\subsubsection{Nuclear magnetic resonance (NMR) analysis of intermediates for the donor half reaction}

A chemical quench $/{ }^{1} \mathrm{H}-\mathrm{NMR}$ method for the quantitative determination of intermediates distribution in the ThDP dependent enzyme reactions has been used extensively since it was developed in 2003 (Kluger and Tittmann, 2008; Tittmann et al., 2003). In order to analyse which intermediates accumulate in the donor half reaction of human TK as well as the effect of LBHB-related single mutations, NMR measurements were measured according to (Mitschke et al., 2010; Tittmann et al., 2003) with defined incubation time. Under the condition that the donor F6P is at the saturation condition and the acceptor is absent, three intermediates were observed on the wild type enzyme, namely the F6P-ThDP covalent adduct (72\%), DHEThDP $(12 \%)$ and free ThDP (16\%, indication of Michaelis complex). NMR spectra of $h$ TK E160Q and E160A reveal identical intermediates distribution that only F6P-ThDP accumulates under the equilibrium condition. In addition, a small fraction of DHEThDP intermediate was observed for the reaction of wild type while barely no such intermediate exists for the reactions of E160Q and E160A. Reactions of human TK E366Q and E165Q with F6P for $30 \mathrm{~s}$ incubation time before acid-quench reveal only the existence of the Michaelis complex (free ThDP) and barely no covalent adduct, suggesting very low catalytic activity for these two variants which is consistent with the steay-state kinetic results. NMR measurement of these two variants for an incubation time of $30 \mathrm{~min}$ with F6P before acid quench have shown that the covalent F6P-ThDP gradually accumulates, indicating that although the enzymes are almost dead but still can perform their catalytic function in a very slow manner. 


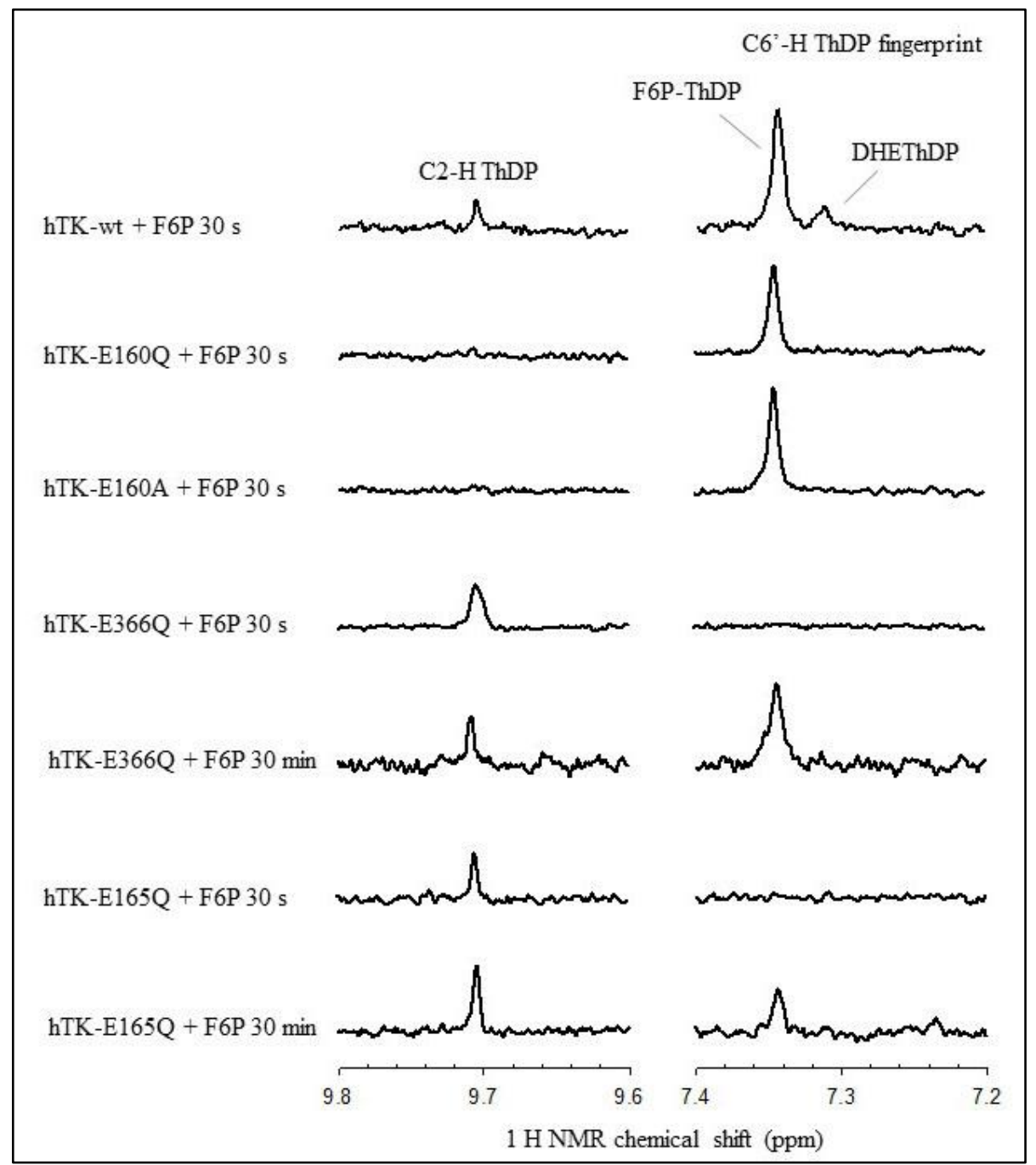

Fig 31: ${ }^{1} \mathrm{H}-\mathrm{NMR}$ based analysis of the intermediate distribution at equilibrium after reaction of human TK wild type and LBHB-related active site variants with physiological substrate F6P were acid quenched and isolated. The characteristic $\mathrm{C} 2-\mathrm{H}$ and $\mathrm{C} 6{ }^{\prime}-\mathrm{H}$ proton signals of ThDP and chemically and chemo-enzymatically synthesized intermediates were used as standards. For the DHEThDP carbanion/enamine intermediate, the $\mathrm{C} 2 \alpha$ protonated conjugate acid form is detected due to the use of strong acid ( $\mathrm{pH} \mathrm{0.75).} \mathrm{The} \mathrm{used} \mathrm{protein} \mathrm{and} \mathrm{incubation} \mathrm{time} \mathrm{for} \mathrm{each} \mathrm{measurement} \mathrm{are}$ indicated in front of the NMR spectra. 


\subsection{Capture the post-cleavage intermediate dihydroxyethyl thiamine diphosphate (DHEThDP) with non-phosphorylated substrates xylulose and fructose}

After the cleavage of the $\mathrm{C} 2-\mathrm{C} 3$ scissile bond on the donor substrate thiamine diphosphate intermediate, one molecule of aldehyde phosphate will be released which leads to the formation of a C2-carbanion, namely dihydroxyethyl thiamine diphosphate (DHEThDP). It potentially resonances with an enamine form through electronic delocalization into the thiazolium ring of ThDP. This $\mathrm{C} 2-\alpha$ carbanion/enamine intermediate plays a pivotal role not only in TKs but also in ThDP-dependent enzyme superfamily (Kluger and Tittmann, 2008).

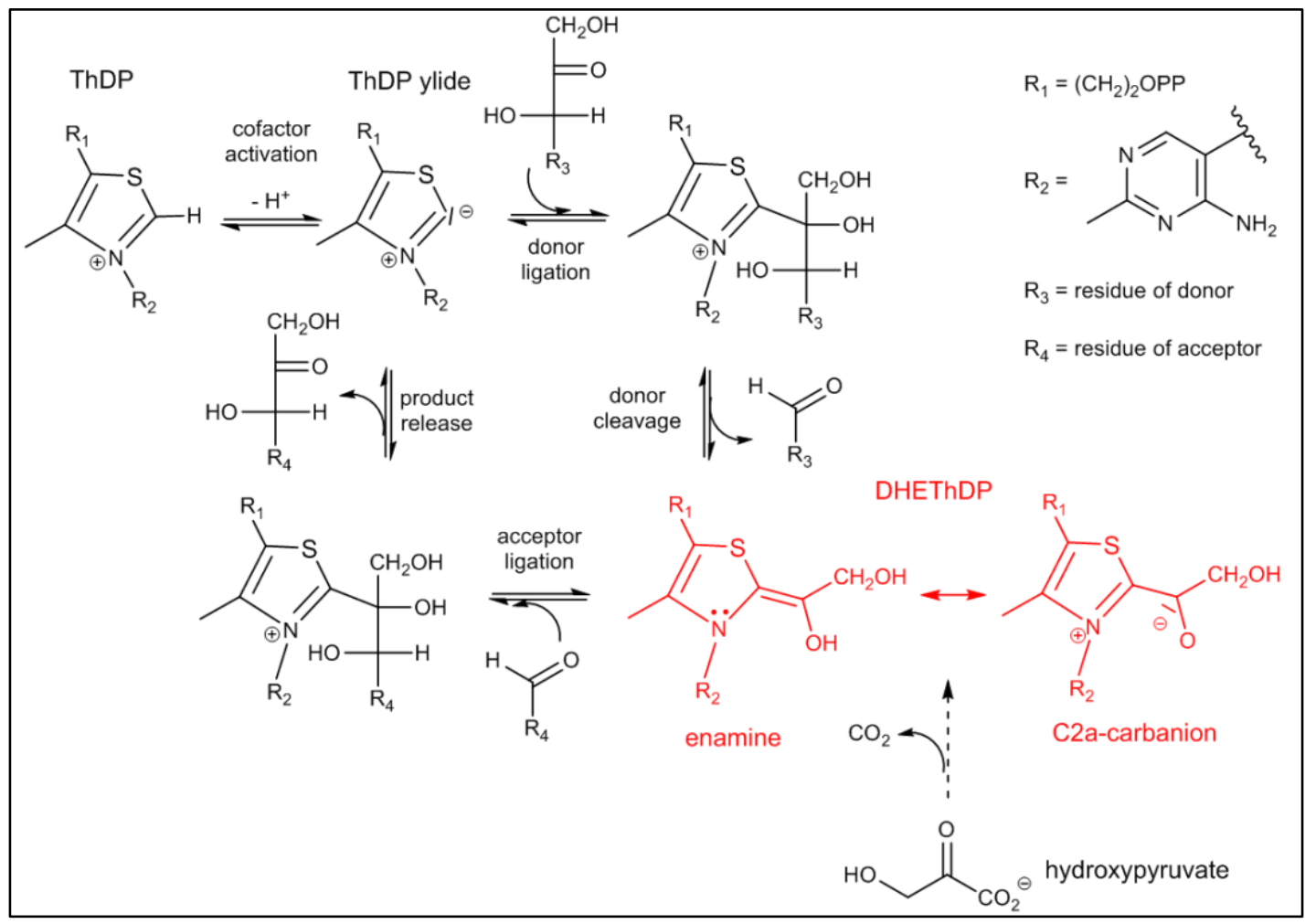

Fig 32. Two routes for the formation of the covalent DHEThDP intermediate. Minimal reaction schemes for transketolase using physiological substrates as well as the artificial substrate HPA are shown. The central DHEThDP intermediate is formed in either pathway and is highlighted in red. This figure was prepared according to (Kluger and Tittmann, 2008) with minor modifications. 


\subsubsection{Spectroscopic and kinetic analysis of the formation of DHEThDP intermediate by using non- phosphorylated sugars}

In our studies, we have found that the non-phosphorylated sugars fructose and xylulose could also serve as the ketol donor substrates for the formation of DHEThDP intermediate. In order to quantitatively analyse the half reaction with fructose and xylulose as the ketol donor, an acid quench isolation of reaction intermediates was used and measured by one-dimensional 1H NMR spectroscopy (Tittmann et al., 2003). This method has been applied on numerous ThDP-dependent enzymes and could monitor a variety of intermediates involved in the reaction scheme.

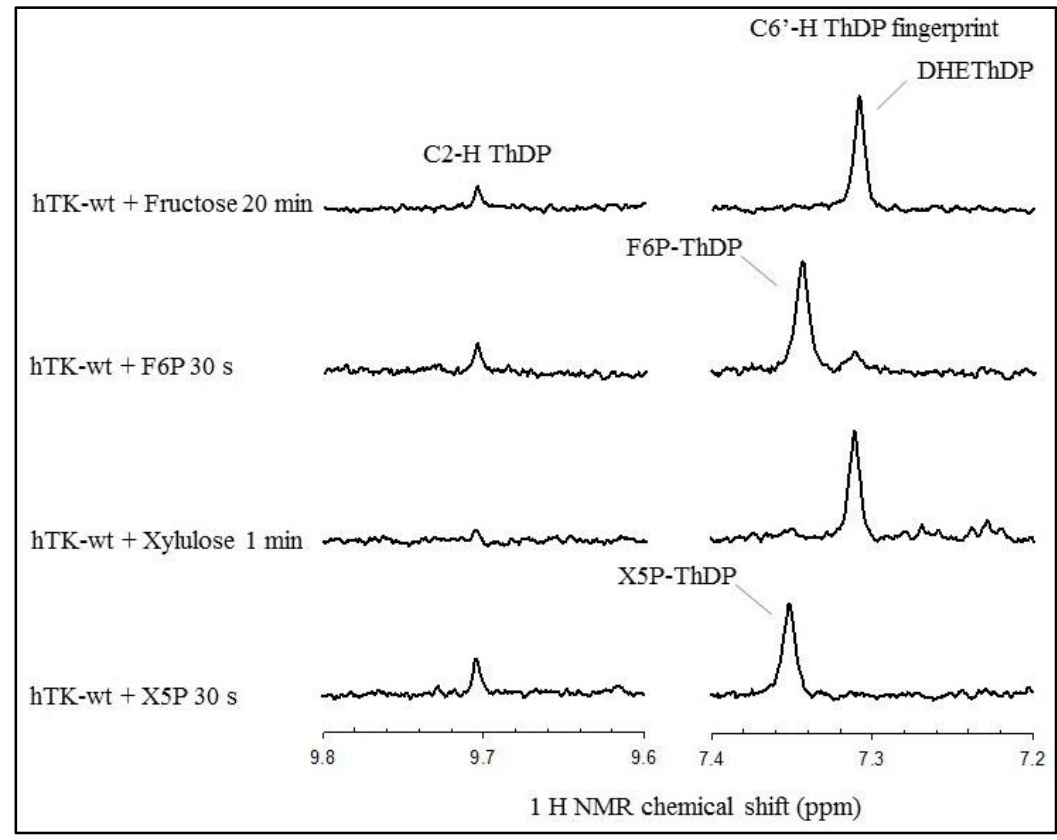

Fig 33: ${ }^{1} \mathrm{H}-\mathrm{NMR}$ based analysis of the intermediate distribution at equilibrium after reactions of human TK wild type with physiological substrates F6P/X5P and non-phosphorylated sugars fructose/xylulose were acid quenched and isolated. The characteristic $\mathrm{C} 2-\mathrm{H}$ and C6'-H proton signals of ThDP and chemically and chemo-enzymatically synthesized intermediates were used as standards. For the DHEThDP carbanion/enamine intermediate, the $\mathrm{C} 2 \alpha$ protonated conjugate acid form is detected due to the use of strong acid ( $\mathrm{pH}$ 0.75). The used substrates (200 mM fructose/xylulose and $50 \mathrm{mM}$ F6P/X5P) and the corresponding incubation time for each measurement are indicated in front of the NMR spectra.

By using the $\mathrm{C} 2-\mathrm{H}$ proton signal of $\mathrm{ThDP}$ and $\mathrm{C} 6$ '- $\mathrm{H}$ signal of chemically synthesized DHEThDP as standards, the relative concentrations of the intermediates could be readily estimated. For the reaction of $h \mathrm{TK}$ with fructose incubated at $4{ }^{\circ} \mathrm{C}$ for 20 min we could quantify 
$85 \%$ DHEThDP intermediate at $7.31 \mathrm{ppm}$ and $15 \%$ un-substituted ThDP. Interestingly, under such conditions barely no covalent substrate-ThDP adduct is detected, which clearly indicates that the equilibrium of the reaction has shifted towards the cleaved intermediate when the phosphate group is absent on the substrate. For comparison, the reaction of F6P with human TK wild type reveals the abundant accumulation of covalent F6P-ThDP intermediate (72 \% at $7.31 \mathrm{ppm})$ and very small amount of the cleaved intermediate DHEThDP (12\%). For the reaction of xylulose incubated at $4{ }^{\circ} \mathrm{C}$ for $1 \mathrm{~min}$, nearly all the active sites of enzyme are occupied by the DHEThDP intermediate ( $95 \%)$ and only minor free ThDP is detected $(<5$ $\%)$. This result suggests that reaction with the five-carbon sugar xylulose is faster than that of the six-carbon counterpart fructose, which is further revealed by the stopped-flow analysis shown in the following chapters. Reaction of X5P with human TK shows only the accumulation X5P-ThDP intermediate (75\% at $7.35 \mathrm{ppm})$ and free ThDP $(25 \%)$.

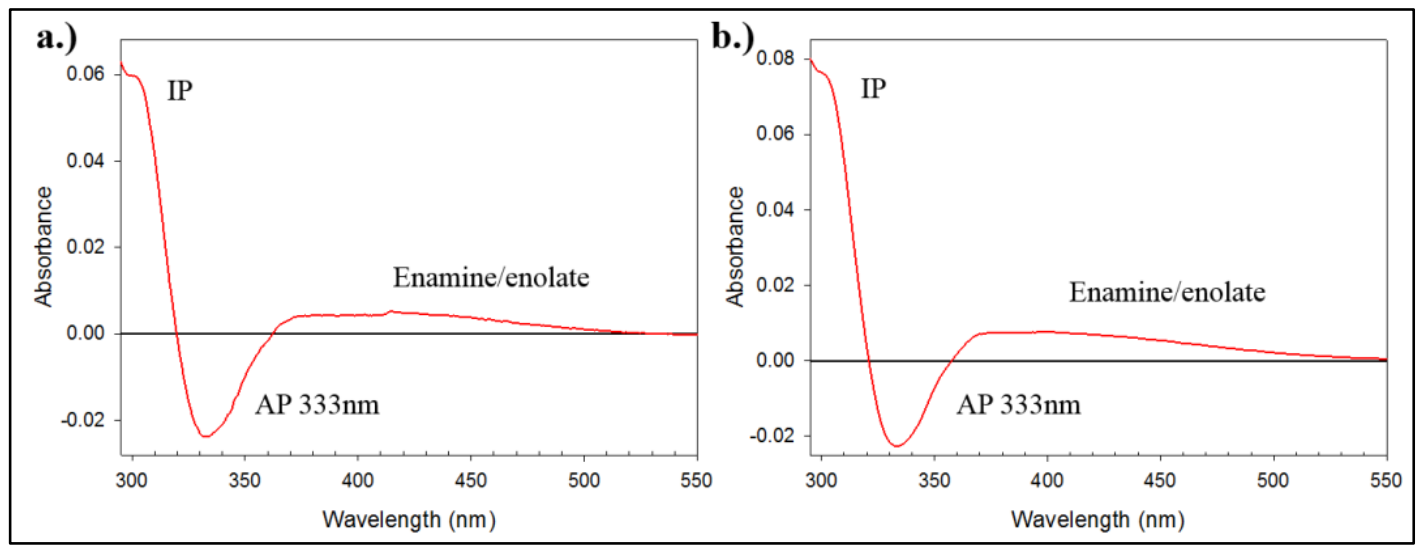

Fig 34: UV-Vis difference spectra of $\boldsymbol{h}$ TK with non-phosphorylated sugars. Reaction of $2 \mathrm{mg} / \mathrm{ml}$ $h \mathrm{TK}$ wt with $25 \mathrm{mM}$ substrate in $50 \mathrm{mM}$ Glycylglycine, $5 \mathrm{mM} \mathrm{CaCl}_{2}$, pH 7.6 at $20^{\circ} \mathrm{C}$. a). Xylulose incubated for $1 \mathrm{~min}$; b). Fructose incubated for $20 \mathrm{~min}$.

In addition to acid quench/1H NMR analysis, the accumulation of the DHEThDP intermediate is further supported by a spectral signature derived from the UV-Vis difference spectrum. Previous studies using HPA as ketol donor have assigned a representative broad band from 380-500 $\mathrm{nm}$ as the enamine form. As Shown in (Lüdtke, 2012), the AP form (320-325 nm) decreases when HPA is covalently added to ThDP, leading to the increase of IP (295-300 nm) form and the enamine form. A very similar spectrum could be observed when the nonphosphorylated sugars fructose and xylulose were used as donor substrates (Fig 34). The entire electric character of the active site has changed due to the absence of the phosphate moiety, the AP band is clearly red-shifted for about $5 \mathrm{~nm}$ and has the maximal absorbance at $333 \mathrm{~nm}$. 
The change transfer band between enamine and six-membered cofactor ring could be observed on both substrates. Although the lack of spectral characteristic of the third protonation state form $\mathrm{APH}^{+}$hampers the analysis of the protonation state of cofactor's six-member ring, previous studies of $E c$ TK have assigned the IP form as the predominant form for the enamine intermediate in this stage (Lüdtke, 2012).

\subsubsection{Structure determination of the DHEThDP intermediate in $h \mathrm{TK}$ by using the non-phosphorylated sugars}

In order to trap the DHEThDP intermediate we tried several times and finally could successfully determine the X-ray structures for $h \mathrm{TK}$ with relatively high occupancy by using non-phosphorylated sugars xylulose and fructose. Although the conditions as used in solution for the NMR intermediates distribution measurement can not be directly applied to the crystalline state, it can provide guidance about the soaking length for the respective substrate. For the 5-carbon sugar xylulose, we have found that the captured DHEThDP intermediate has an occupancy around 70-80 \% when crystals were soaked with $200 \mathrm{mM}$ xylulose for $2 \mathrm{~min}$. We have determined the crystal structure for xylulose at the resolution of $1.5 \AA$ with reasonable statistics $\left(\mathrm{R}_{\text {work }}=14.42 \%, \mathrm{R}_{\text {free }}=17.76 \%\right)$, the electron density extending from the $\mathrm{C} 2$ position of thiazolium ring into the active site clearly indicates the accumulation of the DHEThDP intermediate. For the 6-carbon sugar fructose, DHEThDP intermediate has been observed at the active site with slightly lower occupancy (60-70\%) when crystals were soaked with 200 $\mathrm{mM}$ fructose for $10 \mathrm{~min}$. Similar resolution with reasonable statistics $\left(1.5 \AA, \mathrm{R}_{\mathrm{work}}=15.69 \%\right.$, $\mathrm{R}_{\text {free }}=18.10 \%$ ) of this structure was achieved. To our surprise, the leaving groups, namely Dglyceraldehyde and D-erythrose which are released after the $\mathrm{C} 2-\mathrm{C} 3$ scissile bonds are cleaved, could also be observed at the entrance of the active site of $h \mathrm{TK}$.

In order to estimate the bond length to determine the chemical nature of the intermediate, a weak refinement restrains for the DHEThDP intermediate molecule was used by PHENIX (Adams et al., 2010). Although the refined bond lengths are therefore model-biased because of the input parameters, after more than 25-30 cycles of refinement the obtained model and the electron density map should be gradually converging and a trustful model will be obtained by the end. The structures only have medium resolution (1.5 ̊̊), but this should be enough to distinguish between single bond and double bond.

By carefully analysing the bond lengths for the carbanion-enamine intermediate trapped in $h$ TK using non-phosphorylated sugar xylulose (Fig 35), we have revealed that the C2-C2 $\alpha$ bond $(1.35 \pm 0.02 \AA)$ is significantly shorter than a typical C-C single bond ( $1.54 \AA$ ) but quite 
similar to a typical C-C double bond (1.34 $\AA$ ) (Allen et al., 1987). This clearly supports the predominant enamine character of the intermediate. Another thing needs to be mentioned is that the $\mathrm{C} 2 \alpha-\mathrm{O} 2 \alpha$ bond length is around $1.35 \AA$, a number lies between the ranges of typical C-O single bond (1.43 $\AA$ ) and C-O double bond (1.24 $\AA$ ), suggesting partially double bond character. These features are consistent with previously obtained DHEThDP intermediate structures of $E c$ TK soaked with donor substrate $\beta$-hydroxypyruvate (HPA) (Lüdtke, 2012). An analogous observation for bond length could also be seen for the DHEThDP intermediate captured in $h \mathrm{TK}$ by using the 6-carbon sugar fructose as donor substrate (Fig 62). The only observable difference is that the $\mathrm{C} 2 \alpha-\mathrm{O} 2 \alpha$ bond length is around $1.51 \AA$, being larger than the typical C-O single bond. Lower occupancy of the dihydroxyethyl moiety in this structure might hamper the determination of the exact atom positions.

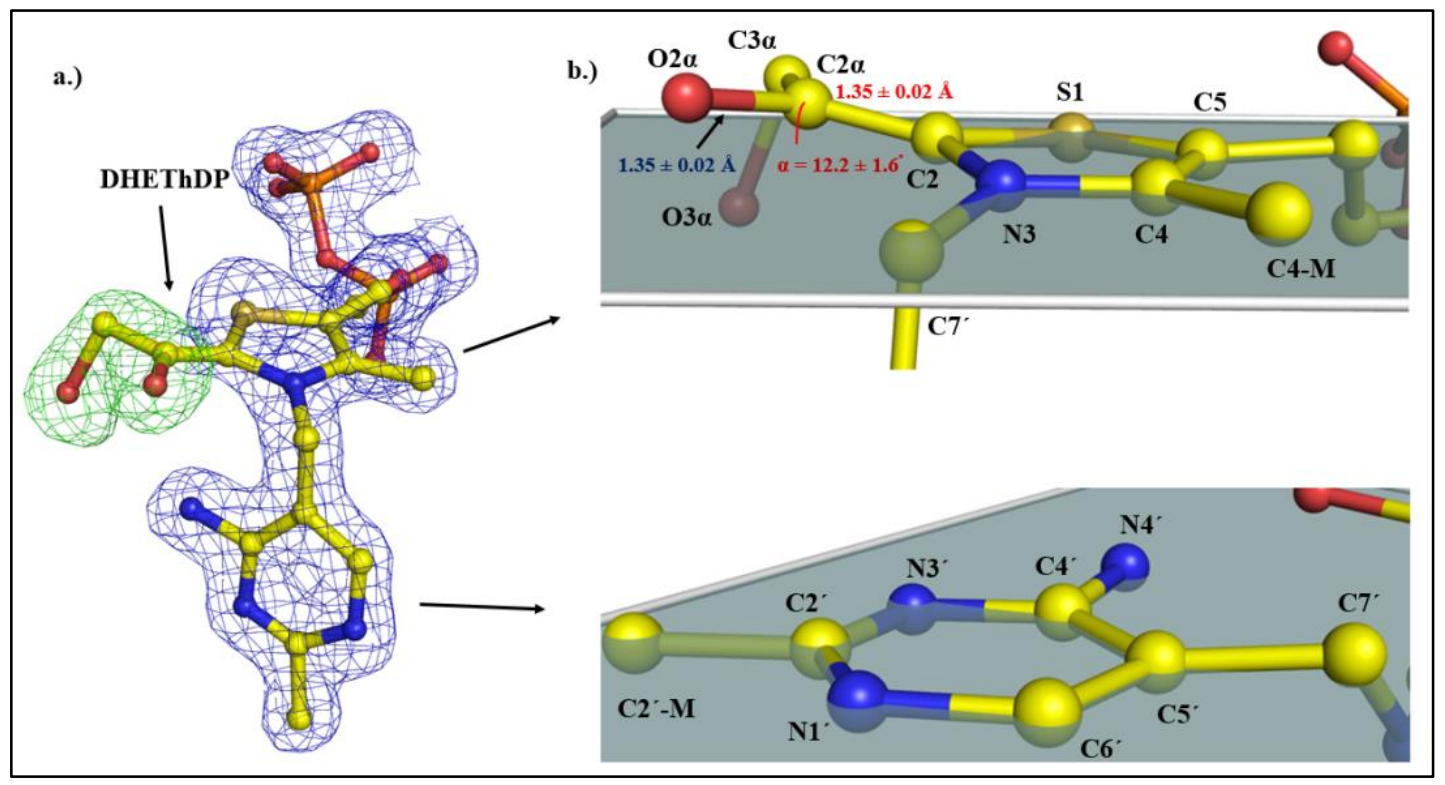

Fig 35: Detailed view of the carbanion-enamine intermediate trapped in $h \mathrm{TK}$ by using xylulose at a resolution of $1.5 \AA$. a). The DHEThDP intermediate is shown in ball-stick representation with the 2Fo-Fc map contoured at $1 \sigma$ level (blue) shown for ThDP moiety and the Fo-Fc map contoured at $3 \sigma$ level (green) shown for the dihydroxyethyl moiety of DHEThDP intermediate. b). Close-up of the intermediate that highlights the deviations from planarity of the thiazolium moiety (top) and aminopyrimidine moiety (bottom) by shown with auxiliary planes. Selected atoms and bond lengths are labelled.

A very surprising and unexpected structural feature of the intermediate (Fig 35b, top) is associated with $\mathrm{C} 2-\mathrm{C} 2 \alpha$ bond that links ThDP cofactor and the dihydroxyethyl moiety. As the thiazolium ring is aromatic and its $\mathrm{C} 2$ atom is expected to be $\mathrm{sp} 2$ hybridized, this newly formed 
$\mathrm{C} 2-\mathrm{C} 2 \alpha$ bond should stay in the same plane with the thiazolium ring. This planar enamine state of DHEThDP intermediate has been observed in ScTK at a resolution of $1.9 \AA$ (Fiedler et al., 2002). Our results, however, indicate unambiguously an out-of-plane distortion for the C2$\mathrm{C} 2 \alpha$ bond from the thiazolium plane by about $13^{\circ}$ and $24^{\circ}$ for $h \mathrm{TK}$ xylulose and fructose structures respectively. The six-membered ring, aminopyrimidine of the DHEThDP intermediate, also exhibits some unexpected features. Atoms N3', C2'-M, C7' show deviations from the plane formed by atoms $\mathrm{N}^{\prime}, \mathrm{C} 5$ ' and $\mathrm{C} 6$ ' of the ring while other atoms C2', C4' and N4' lie coplanar within the aminopyrimidine ring (Fig 35b, bottom).

\subsubsection{Interactions of the DHEThDP intermediate within the active site of $h \mathrm{TK}$}

The newly formed dihydroxyethyl moiety of the DHEThDP intermediate from both nonphosphorylated sugars is positioned at the active site of $h \mathrm{TK}$ by identical polar interactions which are partially mediated by two water molecules at the same position (Fig. 36). Comparing with the active site architecture of $h$ TK with DHEThDP intermediate obtained using HPA, the two structures are nearly superimposable, indicating the same chemical features of DHEThDP intermediates obtained from different substrates. The $\mathrm{N} 4$ ' atom of the aminopyrimidine ring and the $\mathrm{O} 2 \alpha$ of the dihydroxyethyl moiety show weak interaction, which suggests a mechanistic role for acid-base catalysis or stabilization of charges. Same with the HPA induced DHEThDP intermediate structure, two alternative conformations for the side chain of Gln428 are clearly observable except that the occupancies of these have reversed population. The higher populated conformation (67\%, $72 \%$ in xylulose, fructose induced DHETHDP respectively) shows strong hydrogen bonding interaction (distance $2.49 \AA$ ) with O2 $\alpha$, the lower populated conformation $(33 \%, 25 \%$ in xylulose, fructose induced DHEThDP respectively) could potentially form hydrogen bonding interaction with $\mathrm{O} 1 \alpha$ (distance $2.71 \AA$ and $2.72 \AA$ ). These two interactions indicate Gln428 plays pivotal role in the stabilization of DHEThDP intermediate. In both structures, His77 is stabilized by the phosphate group of cofactor and $\mathrm{O} 1 \alpha$ of the dihydroxyethyl moiety through a water molecule, indicating the potential role for cofactor binding and positioning for this conserved residue (His66 in EcTK) in TKs. 


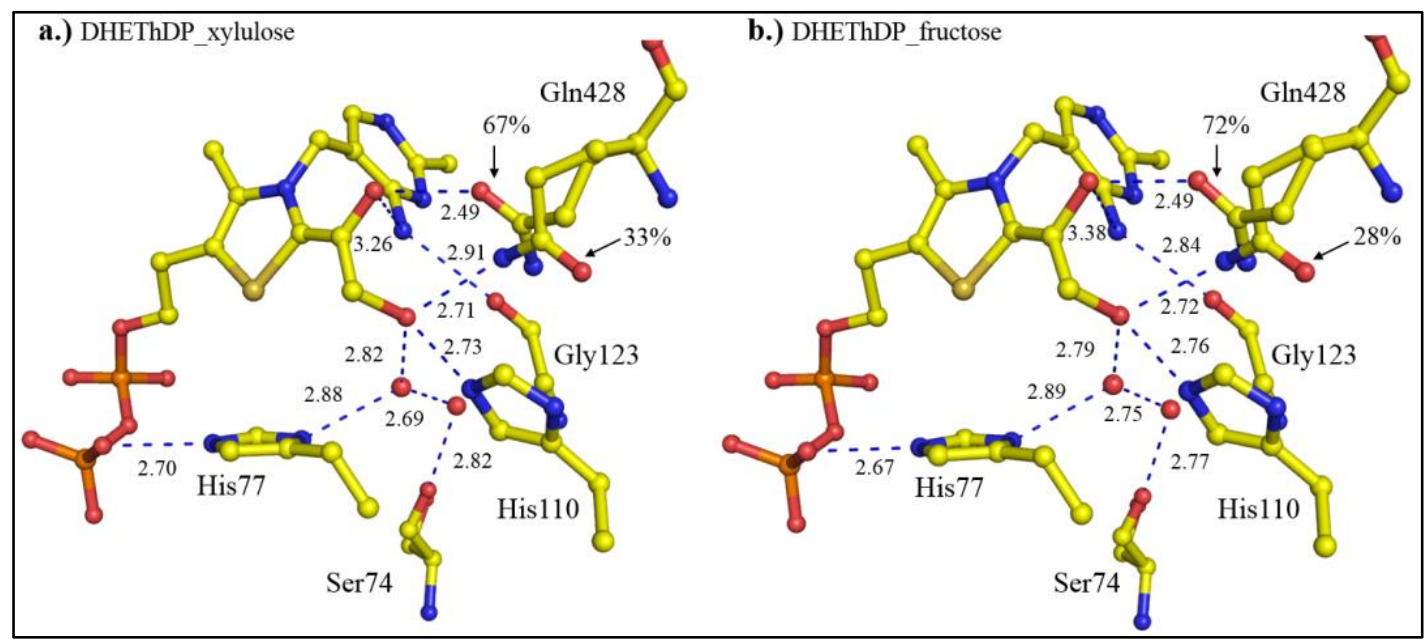

Fig 36: Hydrogen bonding networks of the DHEThDP intermediate formed by using a.) xylulose and b.) fructose within the active site of $\boldsymbol{h T K}$. Active sites residues which form hydrogen bonding interactions (blue dash lines, in $\AA$ ) with the dihydroxyethyl moiety of the DHEThDP intermediate are shown in stick and ball representation. The two water molecules mediating the interactions are shown in red spheres. Note that Glu428 has two alternative conformations (occupancy indicated) in both structures.

One thing needs to be emphasized here is that the $\mathrm{O} 1 \alpha$ of dihydroxyethyl moiety forms a strong hydrogen bonding interaction ( $2.49 \AA$ in both) with the higher populated conformation of Gln428. This strong hydrogen bond could provide repulsive force and lead to the formation of the out-of-plane distortion of the $\mathrm{C} 2-\mathrm{C} 2 \alpha$ bond. In this case, the enzyme active site could provide microenvironment through hydrogen network to trap and stabilize high-energy reaction intermediates. Such transient state could lower the activation energy in the subsequent reaction steps compared with the low-energy planar enamine form. Very similar examples have been observed in transketolase at the donor-ThDP intermediate stage (Asztalos et al., 2007; Lüdtke et al., 2013) as well as other metabolic enyzmes, such as transaldolase (LehwessLitzmann et al., 2011) and pyruvate oxidase (Meyer et al., 2012). Out-of-plane distortion have been observed both in $h \mathrm{TK}$ and EcTK with similar torsion angles and an elongated scissile bond of C2-C3 was observed as well. In transaldolase an authentic Schiff-base intermediate in high-energy conformation was captured by X-ray crystallography, leading to the same conclusion as mentioned above. 


\subsubsection{Capture of the leaving groups D-glyceraldehyde and D-erythrose in $h$ TK}

Native substrates X5P and F6P form a covalent donor-ThDP adduct with the scissile C2-C3 bond elongated about $0.1 \AA$. The phosphate group of native substrates forms strong hydrogen bonding interactions with Arg318, Ser345, His416, Arg474 and four water molecules. Thus, the phosphate group could stabilize the donor-ThDP intermediate by these strong interactions within the active site of $h \mathrm{TK}$. Unlike native substrates, donor-ThDP intermediates formed by the non-phosphorylated sugars couldn't benefit from the strong interactions of the phosphate group with the enzyme's active site, therefore the scissile C2-C3 bond could not be stabilized in the elongated state but is readily cleaved off, leading to the formation of the post-cleavage intermediate DHEThDP and one molecule of D-glyceraldehyde or D-erythrose is released into the active site. Luckily, these cleaved-off products could be captured within the active site at similar position where the phosphates group of native substrates bind.

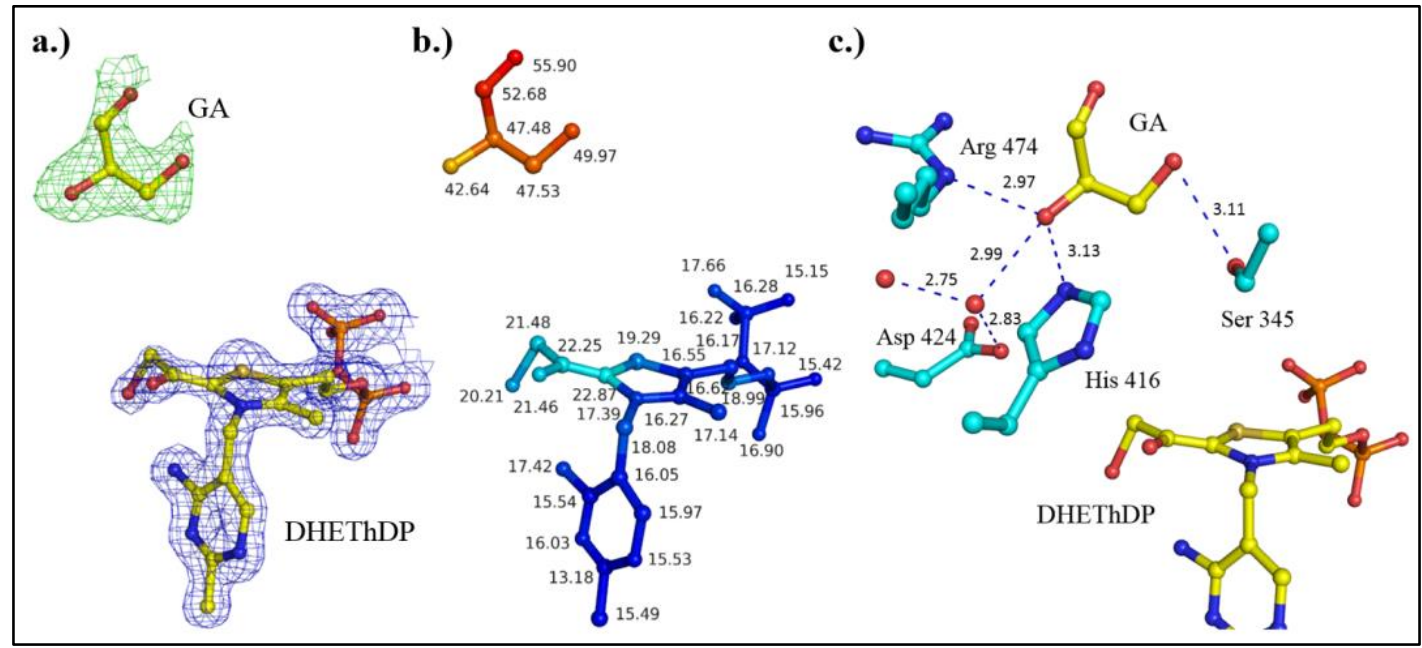

Fig 37: Binding of post-cleavage product D-glyceraldehyde from xylulose to $h$ TK. After breakage of the scissile C2-C3 bond one molecule of D-glyceraldehyde (D-GA) is released and captured at the active site of $h$ TK. a.) D-GA shown with a residue-omitted Fo-Fc map (contoured at $3 \sigma$ level) bound approx. $8 \AA$ away from the DHEThDP intermediate which is surrounded by a 2 Fo-Fc map (contoured at $1 \sigma$ level). b.) Atoms of D-GA and DHEThDP are colour-labelled according to their individual Bfactors with most mobile atoms in red $\left(\approx 50 \AA^{2}\right)$ and less mobile ones in blue (15-18 $\left.\AA^{2}\right)$. c.) Interactions of post-cleavage product D-GA with the active site residues (shown in cyan) are indicated by blue dashed lines with inter-atomic distances.

The leaving product D-GA is positioned by polar interactions with Arg474, Ser345, His416 and Asp424 though a water molecule. These interaction are mainly accomplished by the two hydroxyl groups of D-GA and the lack of hydrogen interactions with the aldehyde group 
renders its flexibility. Detailed analysis of the atomic B-factors reveals the 2-3 fold increased flexibility of the 3-carbon product compared to the tightly integrated cofactor. Moreover, atomic B-factors for the dihydroxyethyl moiety of the DHEThDP intermediate are approx. 20 $\%$ higher relative to the ThDP body of cofactor, suggesting an increased flexibility for the dihydroxyethyl moiety. Closer inspection of the atomic B-factors on the 3-carbon product DGA also suggests a higher flexibility for the aldehyde group (15\% higher than the rest part).

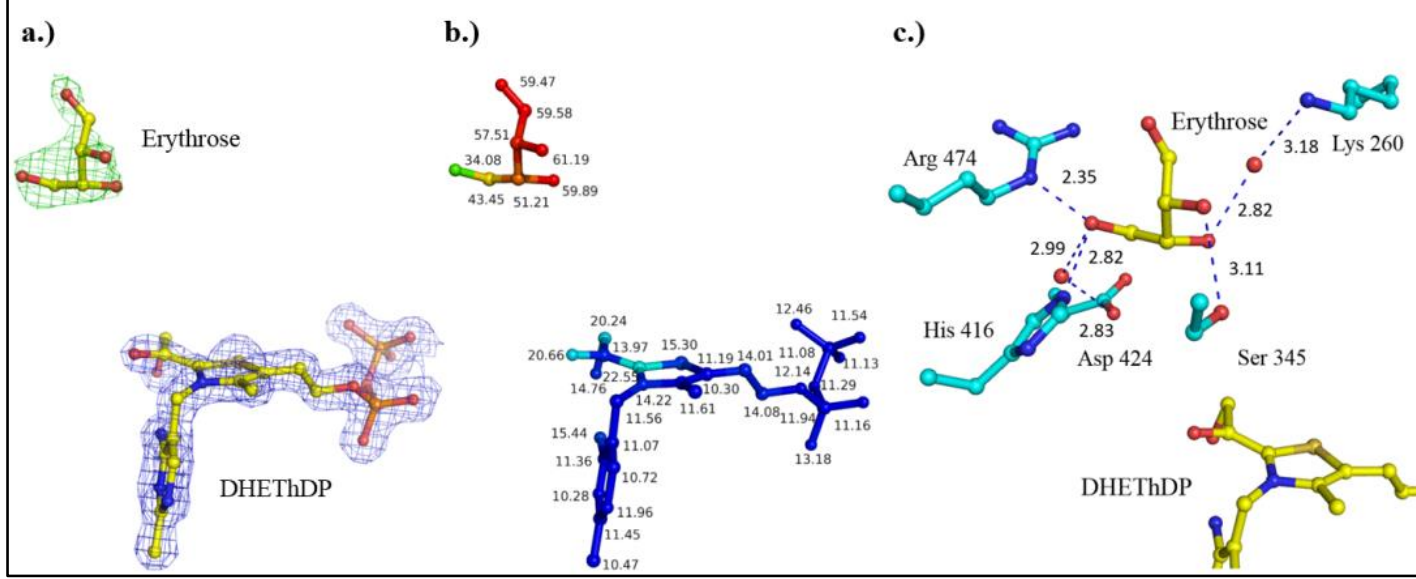

Fig 38: Binding of post-cleavage product D-erythrose from fructose to $\boldsymbol{h T K}$. After breakage of the scissile $\mathrm{C} 2-\mathrm{C} 3$ bond one molecule of D-erythrose is released and captured at the active site of $h \mathrm{TK}$. a.) D-erythrose shown with a residue-omitted Fo-Fc map (contoured at $2.5 \sigma$ level) bound approx. $8 \AA$ away from the DHEThDP intermediate which is surrounded by a $2 \mathrm{Fo}-\mathrm{Fc}$ map (contoured at $1 \sigma$ level). b.) Atoms of D-GA and DHEThDP are colour-labelled according to their individual B-factors with most mobile atoms in red $\left(\approx 60 \AA^{2}\right)$ and less mobile ones in blue (11-14 $\left.\AA^{2}\right)$. c.) Interactions of post-cleavage product D-erythrose with the active site residues (shown in cyan) are indicated by blue dashed lines with inter-atomic distances.

The cleaved-off product D-erythrose from the 6-carbon sugar D-fructose could also be captured at the same location and positioned by nearly identical polar interactions. Different from D-GA, one more lysine residue (Lys260) participates in the stabilization of the 2-OH group though a water molecule. D-erythrose shows weaker electron density (contour level $2.5 \sigma$ in Fig 38) compared to that of D-GA (contour level $3 \sigma$ in Fig 37) even the same soaking time is used for both structures, suggesting a slower reaction velocity for the 6-carbon sugar fructose. The increased flexibility for the D-erythrose relative to the cofactor, as well as the aldehyde group of D-erythrose relative with its rest part, are clearly indicated by their atomic B-factors, which is identical to that of the xylulose structure. In addition, the release of Derythrose could also be confirmed by a photodiode array based stopped-flow experiment (Fig $63)$. 


\subsubsection{Single-mixing stopped-flow analysis of the donor half reaction using non- phosphorylated sugars}

In the LBHB chapter, the microscopic kinetic constants for the native donor half reaction have been measured by stopped-flow spectroscopy using F6P, but the reaction velocities for transketolase using the non-phosphorylated sugars still remain unknown at the moment. To analyse the formation of the DHEThDP intermediate kinetically, stopped-flow spectroscopy experiments were performed in the absence of the acceptor substrate. Based on the UV-Vis difference spectra shown before (Fig 34), two wavelengths were chosen to monitor the progress of the formation or depletion of the protonation state of cofactors' six-membered ring (303 nm for IP form, $333 \mathrm{~nm}$ for AP form). Another experiment was performed at $400 \mathrm{~nm}$ to monitor the generation of the enamine/enolate intermediate. For reasons of substrate availability, reaction velocities for xylulose were only determined for the AP formation.

Single mixing stopped-flow results reveal that the formation of the DHEThDP intermediate from non-phosphorylated sugars is a very slow process (Fig 39). All reaction transients indicate a single molecular process and the rate constants could be obtained by fitting the curve according to a single exponential equation. By plotting the observed reaction velocity against the utilized fructose concentrations, linear dependencies for AP depletion and IP or enamine/enolate formation are observable. The rate constants for these three individual processes at any given donor substrate concentration are identical, strongly suggesting same molecular origin. In addition, compared with the native substrates, 100-fold higher fructose concentrations are employed in order to obtain measurable reaction transients. The lack of the phosphate group makes the substrate not targetable by the active site, therefore extremely high concentration are necessary for the substrate to diffuse into the active site instead of specific recognition. The diffusion velocity is proportional to concentration, resulting in the linear dependency.

In order to calculate the dissociation constant $\left(K_{\mathrm{d}}\right)$, amplitude of the obtained reaction transients were plotted against corresponding fructose concentrations (Fig 64), a hyperbolic dependency was observable and the $K_{\mathrm{d}}$ value calculated to $36 \mathrm{mM}$. This millimolar range dissociation constant value again confirms the weak binding of non-phosphorylated fructose to $h$ TK. In addition, the full amplitude of enamine/enolate measured at $400 \mathrm{~nm}$ only take up $1 / 10$ of AP which is consistent with the UV-Vis difference spectra.

Similar experiments performed with EcTK at $322 \mathrm{~nm}$ (depletion of AP) revealed a biphasic behaviour, indicating that a two-molecular process is monitored (Fig 65). The second phase might be explained by the cofactor flexibility property which was previously revealed by highresolution X-ray structures (Lüdtke, 2012). Like the native substrate reaction, formation of the 
DHEThDP intermediate by the non-phosphorylated sugar fructose exhibits 40 -fold increased velocity over that of $h \mathrm{TK}$.
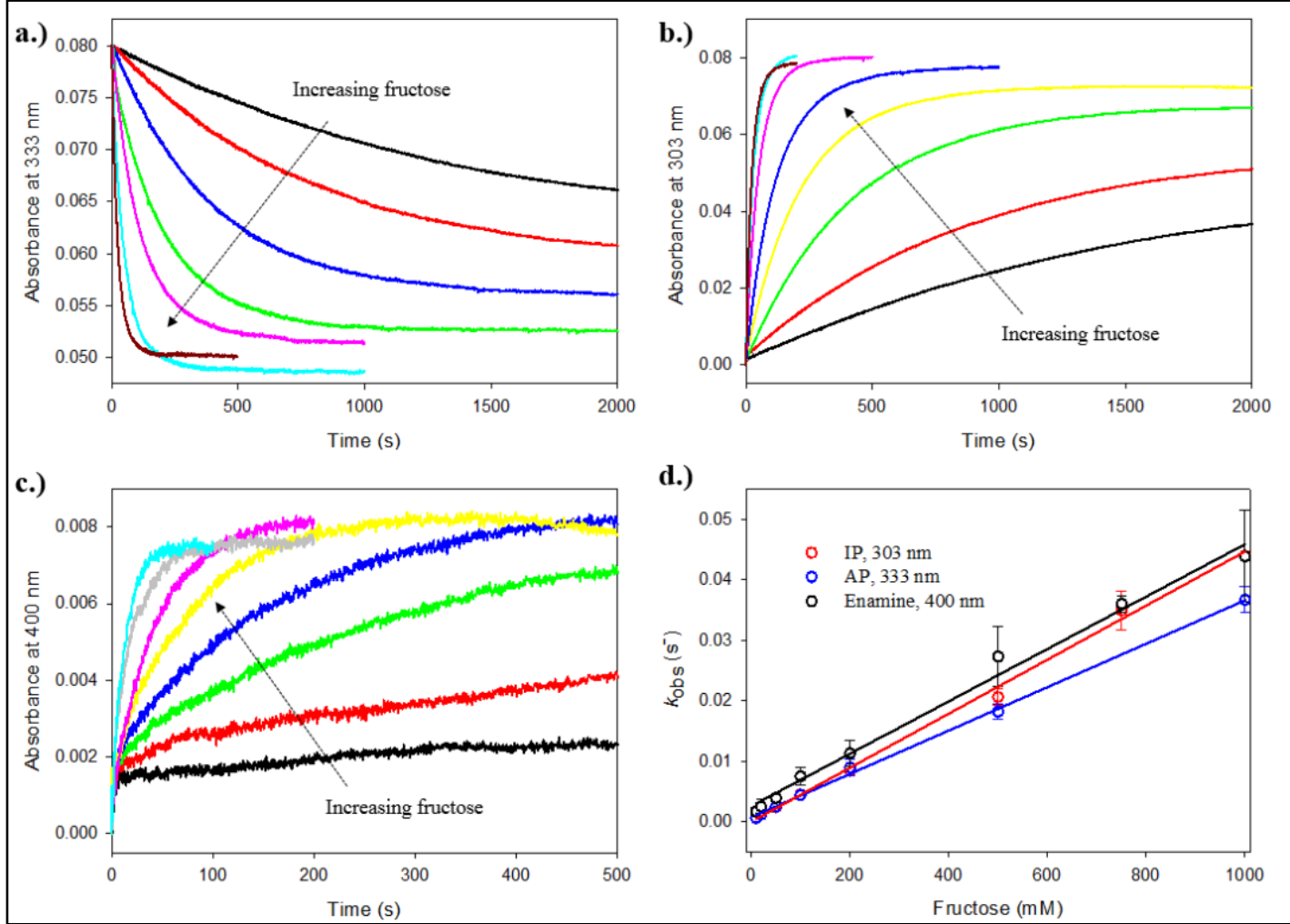

Fig 39: Kinetic analysis for the reaction of the formation of DHEThDP intermediate in $h$ TK with the non-phosphorylated sugar fructose. Single-mixing stopped-flow absorbance transients $(10 \mathrm{~mm}$ path length) for the reaction of $5 \mathrm{mg} / \mathrm{ml} h \mathrm{TK}, 5 \mathrm{mM} \mathrm{CaCl}_{2}, 50 \mathrm{mM}$ glycylglycine (pH 7.6) with different concentrations of fructose at $4{ }^{\circ} \mathrm{C}$. a.) Progress curves for the formation of IP form at $303 \mathrm{~nm}$. b.) Progress curves for the depletion of AP form at $333 \mathrm{~nm}$. c.) Progress curves for the formation of enamine form at $400 \mathrm{~nm}$. d.) Reaction constants were determined according to a single exponential equation (A $=\mathrm{A} 1 * \mathrm{e}^{-k^{*} t}+$ offset$)$ and then plot against the utilized fructose concentration. The velocities for all three chemical processes exhibit linear properties.

The five-carbon sugar D-xylulose shows around 15-fold increased reaction efficiency for the depletion of the AP form compared with that of the six-carbon donor (fig 40). Although the data for the formation of the IP from and the enamine are not available due to substrate rarity, we could deduce that these two process should exhibit similar behaviour based on the data from fructose. The shorting of one carbon enables the substrate to enter the active site more easily, thus promoting the possibility for the nucleophilic attack on the carbonyl group of xylulose from the ThDP C2 carbanion. The smaller leaving group (3-carbon compared with 
4-carbon) also makes the diffusion and cleavage reaction more readily due to less hydrogen bonding interactions.

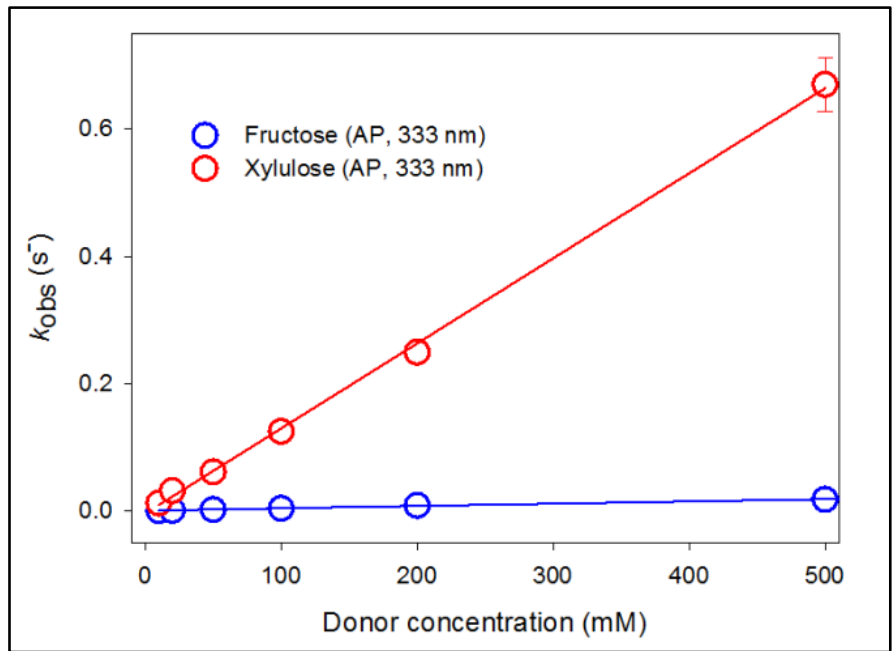

Fig 40: Comparison of the rate constants for AP formation using fructose and xylulose measured by single-mixing stopped-flow spectroscopy. Reaction conditions refer to Fig 39. Please note that at any given substrate concentration 5-carbon sugar xylulose exhibits 15-fold increased reaction velocity relative to that of 6-carbon sugar fructose.

3.2.6. Double-jump stopped-flow analysis for the reactivity of the post-cleavage DHEThDP intermediate from non-phosphorylated sugars with native acceptor R5P

In transketolase, the post-cleavage carbanion-enamine intermediate acts as nucleophile which attacks the aldehyde group of the acceptor in the second half of the reaction. Therefore the enzyme has to evolve to avoid protonation of the carbanion-enamine. From structural perspectives, there are no amino acids found close to the C $\alpha$ atom of DHEThDP which could potentially catalyse the protonation reaction. Pyruvate decarboxylase (PDC) however contains one glutamate (Glu477) residue positioned to participate in the proton transfer, which is part of the catalytic mechanism of PDC (Lobell and Crout, 1996). In the obtained crystal structures discussed above, the DHEThDP intermediates could not be differentiated from the protonation state. To test the reactivity of the carbanion-enamine intermediate obtained from nonphosphorylated sugars, sequential stopped-flow spectroscopy was employed to quantitatively measure the reaction with the native acceptor substrate R5P.

To get a general view of the DHEThDP intermediate depletion after adding R5P, UV-Vis difference spectra was first calculated. The depletion of two bands were observable with one 
centred at $308 \mathrm{~nm}$ and another one at $440 \mathrm{~nm}$. Previous studies with another artificial substrate HPA (Lüdtke, 2012) have shown that the depletion of two similar bands (300 and $400 \mathrm{~nm}$ ) have nearly identical kinetic features, indicating the same chemical origin, the depletion of carbanion-enamine, for these two bands. Due to signal-to-noise consideration, $308 \mathrm{~nm}$ was specifically chosen to measure the activity of the DHEThDP intermediate.

After initial mixing of the non-phosphorylated donor substrate fructose with $h \mathrm{TK}$ the DHEThDP intermediate was accumulated on the active site of the enzyme. In the following step the enzyme-bound DHEThDP intermediate was rapidly mixed with the physiological acceptor substrate R5P and the reaction was monitored by a stopped-flow spectrophotometer. A representative reaction transient for this particular rapid reaction is shown in Fig 41. Fitting of the original data points according to a double exponential equation is more reasonable than to a single exponential equation, strongly suggesting that two chemical processes are observable at this particular wavelength. A similar two-phase reaction was observed on E.coli TK using the donor analogue $\beta$-hydroxypyruvate (HPA) (Lüdtke, 2012).

Two possible catalytic mechanisms could explain the biphasic process of the depletion of the DHEThDP intermediate monitored at $308 \mathrm{~nm}$. a.) A controversial hypothesis, namely negative cooperativity, has been suggested for some ThDP-dependent enzymes based on the fact that all ThDP enzymes are functional dimers. The biphasic behaviour therefore could be interpreted as the existence of the structural non-equivalence of the two active sites of $h \mathrm{TK}$. b.) Another reasonable explanation might be that the first fast phase represents the nucleophilic attack of DHEThDP on the aldehyde function of R5P to form the S7P-ThDP intermediate and the second phase monitors the release of the product S7P. This proposal is supported by the sequential stopped-flow kinetics of the enzyme-bound DHEThDP obtained from HPA with the acceptor substrate R5P measured at $300 \mathrm{~nm}$. 


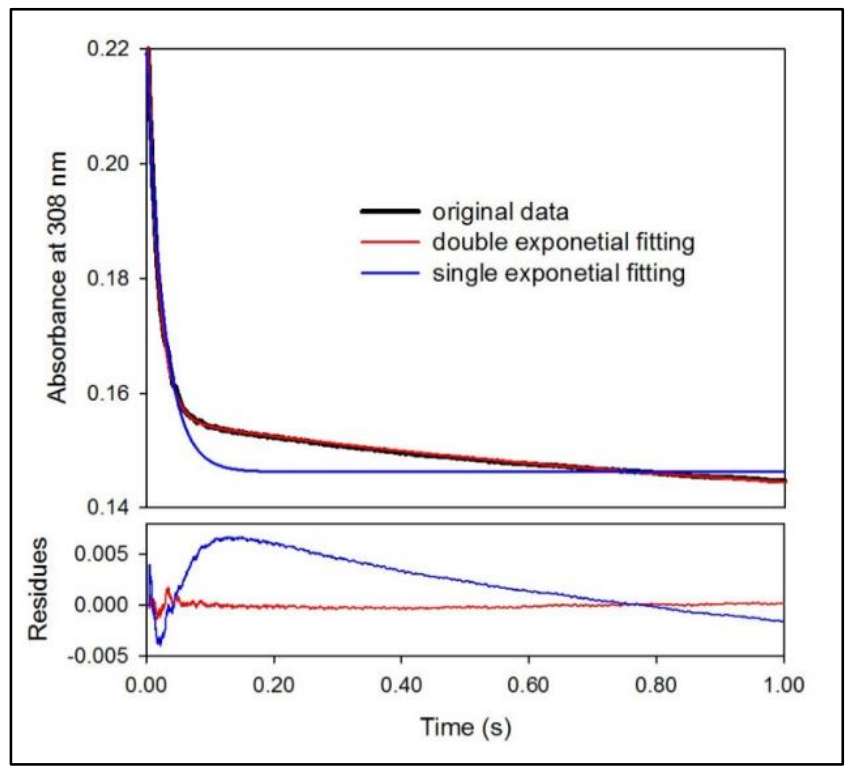

Fig 41: Reaction transients for the depletion of DHEThDP intermediate in $h \mathrm{TK}$ with the acceptor substrate R5P. Double-jump stopped-flow kinetics for the reaction of $8 \mathrm{mg} / \mathrm{ml} h \mathrm{TK}$ which was initially mixed with $1 \mathrm{M}$ fructose for $5 \mathrm{~min}$ to accumulate DHEThDP intermediate with $1 \mathrm{mM}$ R5P in $50 \mathrm{mM}$ glycylglycine, $5 \mathrm{mM} \mathrm{CaCl}$, pH 7.6 at $4{ }^{\circ} \mathrm{C}$. Measurements were conducted with a stopped-flow spectrophotometer at a path length of $10 \mathrm{~mm}$. Fitting of the transient was applied either according to a single exponential equation (blue, $\mathrm{A}=\mathrm{A} 1 \mathrm{e}^{-\mathrm{e}^{* *_{\mathrm{t}}}}+$ offset) or a double exponential equation (red, $\mathrm{A}=$ $\mathrm{A} 1 * \mathrm{e}^{-k 1 * \mathrm{t}}+\mathrm{A} 2 * \mathrm{e}^{-k 2{ }^{*} \mathrm{t}}+$ offset). Residues for both fitting are shown below the reaction transient.

A series of reaction transients were collected with increasing concentrations of R5P. The obtained rate constants for the first fast phase and the second slow phase were plotted against the utilized acceptor R5P concentrations (Fig 42). The velocity of the fast phase could be better fitted according to a Hill equation giving the "S-shaped" sigmoidal curve rather than the classic Michaelis-Menten equation. The Hill coefficient was calculated to a value of $1.67 \pm 0.27$ which clearly indicates the positive cooperative binding for the acceptor R5P. Since $h$ TK comprises of two active sites, allosteric binding could be proposed which is the binding of ligand at one active site would increase the affinity for the binding at another active site. The increased affinity for the substrates leads to a coordinated increase in the velocity of the DHEThDP intermediate depletion at higher substrate concentration. In addition, this kinetic results of positive cooperativity for the binding of R5P at the two active sites of $h \mathrm{TK}$ would rule out the above discussed negative cooperativity suggested to ThDP-dependent enzymes (Frank et al., 2007a; Lindqvist et al., 1992), at least in $h$ TK.

Based on the above discussion, we could assign the observed two phases to the formation of S7P-ThDP intermediate and the liberation of S7P respectively. Calculated reaction velocity for the first fast phase with a $k_{\max }=92.3 \pm 6.1 \mathrm{~s}^{-1}$ indicates that the nucleophilic addition 
reaction is relatively faster than the formation of donor-ThDP intermediate in the first half reaction. But this process is again slower compared with E.coli transketolase $\left(531 \pm 37 \mathrm{~s}^{-1}\right)$. The liberation of S7P from the covalent intermediate in the second phase is not affected by the acceptor substrate concentrations, which is different from the same reaction by taking HPA as the donor substrate. A possible explanation would be that the existence of erythrose, the cleaved-off product from donor fructose, occupies the position where S7P should be released, therefore hinders the liberation of S7P. The lack of the phosphate group probably renders the discrimination of erythrose almost diffusion-controlled which is only relevant with its fixed concentration. In this regard, the reaction of S7P releasing remains constant with very slow rate constant (around $0.8 \mathrm{~s}^{-1}$ ).

In order to chemically confirm the above mentioned two-phase reaction, rapid quenched-flow techniques in combination with ${ }^{1} \mathrm{H}-\mathrm{NMR}$ based intermediate analysis have to be applied on this reaction. Given that quenched-flow experiments are not possible to be performed in the sequential mode, such problems have to be solved technically in the future.

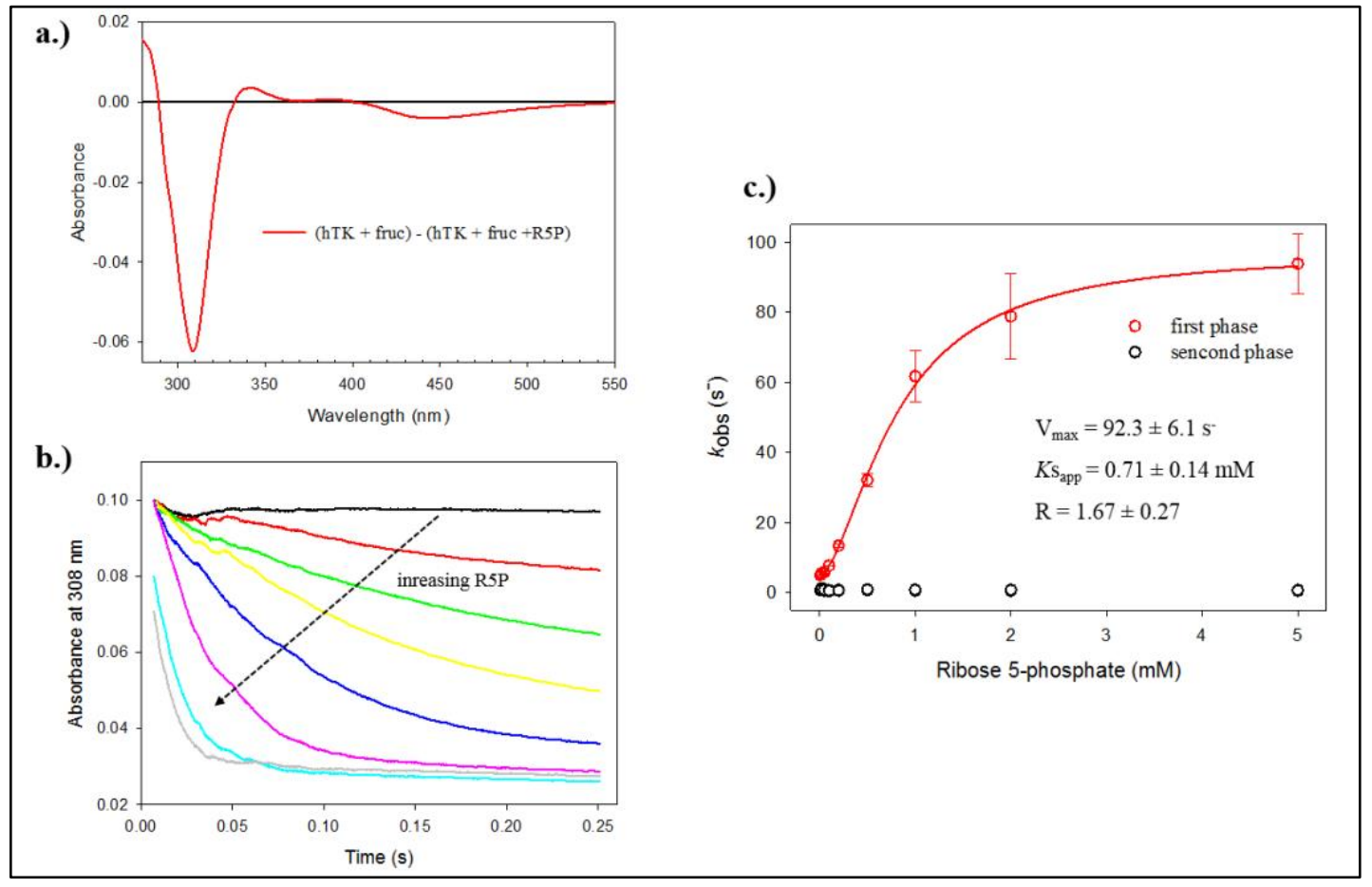

Fig 42: Kinetic analysis for the reaction of DHEThDP intermediate obtained from non-native donor substrate fructose with native acceptor substrate R5P. a.) UV-Vis difference spectra for the depletion of DHEThDP intermediate. $h \mathrm{TK}(2 \mathrm{mg} / \mathrm{ml})$ was first incubated with $25 \mathrm{mM}$ fructose in 50 $\mathrm{mM}$ glycylglycine, $5 \mathrm{mM} \mathrm{CaCl}_{2}, \mathrm{pH} 7.6$ at $25^{\circ} \mathrm{C}$ for $20 \mathrm{~min}$ and a spectra was recorded at this moment, then $5 \mathrm{mM}$ R5P was added and incubated for $2 \mathrm{~min}$ and another spectra was measured, difference spectra was obtained by the substitution of both. b.) Reaction transients for the DHEThDP intermediate 
depletion. $h \mathrm{TK}(8 \mathrm{mg} / \mathrm{ml})$ was initially mixed with $1 \mathrm{M}$ fructose for $5 \mathrm{~min}$ to form the DHEThDP intermediate in $50 \mathrm{mM}$ glycylglycine, $5 \mathrm{mM} \mathrm{CaCl}_{2}, \mathrm{pH} 7.6$ at $4{ }^{\circ} \mathrm{C}$, then acceptor R5P with increasing concentrations were 1:1 mixed and the reaction was recorded by a stopped-flow absorbance spectrometer (path length $10 \mathrm{~mm}$ at $308 \mathrm{~nm}$ ). The top black curve contains $0 \mathrm{mM}$ R5P and shown here as control. Spectra were fitted according to a double exponential equation ( $\mathrm{A}=\mathrm{A} 1{ }^{*} \mathrm{e}^{-k 1 *_{\mathrm{t}}}+\mathrm{A} 2{ }^{*} \mathrm{e}^{-k 2 *_{\mathrm{t}}}+$ offset), the respective rate constants were calculated accordingly. c.) Plotting of the observed rate constants against the utilised concentration of R5P.

\subsubsection{Activation of transketolase by exogenous phosphite dianion for the conversion of non-phosphorylated donor substrates}

Previous studies on orotidine 5'-monophosphate decarboxylase (OMPDC) have revealed that the binding of exogenous phosphite dianion at a remote position from the reacting center could result in a dramatic increase (80000-fold) in the $k \mathrm{cat} / \mathrm{Km}$ for the decarboxylation of shortened substrate without the phosphodianion moiety (Amyes et al., 2005). The non-physiological reactions discussed in this chapter exhibit quite analogous features to OMPDC as the substrates employed only differ for one phosphate group compared to the native substrates. It would be very interesting to explore whether or not transketolases employ a similar reaction mechanism.

In order to investigate the effect of exogenous phosphite dianion for the formation of the DHEThDP intermediate, a single-mixing stopped-flow experiment was performed at $333 \mathrm{~nm}$ to monitor the depletion of AP form similar to the above methods. Increasing phosphite concentrations were supplemented to enzyme and incubated for $1 \mathrm{~min}$ to assure the binding to take place, same amount of phosphite dianion was supplemented to substrate fructose as well to avoid possible dilution when the two components are mixed. Without exogenous phosphite, the depletion of AP form exhibits a rate constant around $0.38 \mathrm{~s}^{-}$which is identical to what we observed before. Increasing phosphite dianion concentration gradually accelerate the formation of DHEThDP intermediate and maximal effect could be achieved with an amount of 2-3 mM phosphite dianion (Fig 43). The reaction velocity could be accelerated up to 0.10 $\mathrm{s}^{-}$(2-3 fold) which is not as remarkable as that of OMPDC, indicating a slightly different mechanism. 


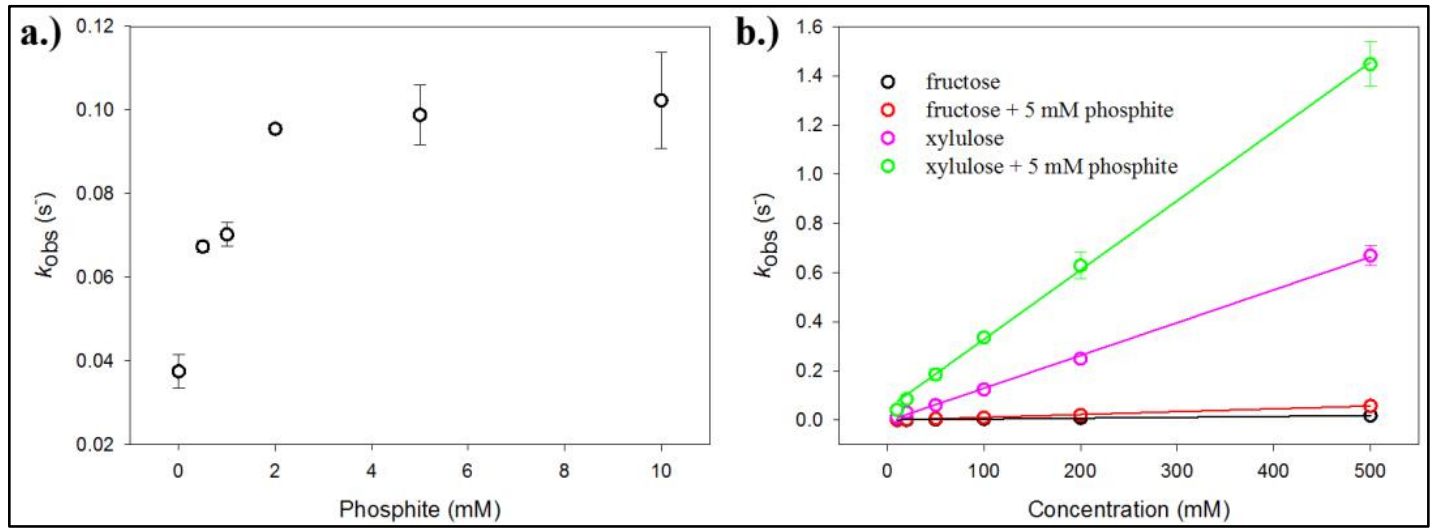

Fig 43: Kinetic analysis of the effect of phosphite dianion for the formation of DHEThDP intermediate by the non-phosphorylated sugars fructose and xylulose. a.) Single-mixing stoppedflow spectroscopy measurement for the reaction of $h \mathrm{TK}(4 \mathrm{mg} / \mathrm{ml})$ with $1 \mathrm{M}$ fructose supplemented with increasing concentration of exogenous phosphite dianion. All materials were dissolved in $50 \mathrm{mM}$ glycylglycine, $5 \mathrm{mM} \mathrm{CaCl}_{2}$, pH 7.6. For specific concentration, both enzyme and substrates were incubated for $1 \mathrm{~min}$ with phosphite dianion before shooting. The reaction was measured at $333 \mathrm{~nm}$ at 4 ${ }^{\circ} \mathrm{C}$ with a path length of $10 \mathrm{~mm}$. b.) Comparison of the reaction velocity for the reaction of $h \mathrm{TK}$ with the non-phosphorylated sugars in the presence and absence of exogenous phosphite dianion. Reaction conditions refer to Fig $39.5 \mathrm{mM}$ phosphite were added both to the enzyme and substrates and incubated for $1 \mathrm{~min}$ before the measurement took place. All measurements were performed as triplicate to avoid random errors.

To further explore the kinetic characteristics with the presence of phosphite dianion for the reaction of DHEThDP intermediate formation, $5 \mathrm{mM}$ phosphite dianion was specifically chosen for the activation of enzyme. Different from our expectation, the presence of two parts of the physiological substrate doesn't restore the enzymatic kinetics to the Michaelis-Menten mode but still exhibits a linear dependency. Therefore the diffusion-controlled manner of reaction doesn't change despite the existence of the activating phosphite dianion. The sixcarbon fructose mediated formation of the DHEThDP intermediate is still 12-fold slower compared with that of the five-carbon counterpart xylulose even in the presence of $5 \mathrm{mM}$ phosphite dianion, confirming identical mechanism for both substrates upon the binding of activating dianion. The rate constant $\left(k_{\mathrm{obs}}\right)$ for the reaction of $500 \mathrm{mM}$ xylulose in the presence of phosphite dianion reached $1.4 \mathrm{~s}^{-}$and can still increase if more substrates are available. This behaviour is comparable with the previous steady-state and transient state kinetic $\left(k_{\mathrm{cat}}=2.84\right.$ $\pm 0.53 \mathrm{~s}^{-}$for steady-state kinetics, $k_{\mathrm{obs}}=2.62 \pm 0.05 \mathrm{~s}^{-}$for the pre-steady-state kinetics of F6PThDP formation). 
Since the binding of the phosphite dianion to the active site could enhance the DHEThDP intermediate formation from non-phosphorylated sugars, it is reasonable to assume that binding of the activating phosphite dianion, even at a remote position from the active site, would alter the micro-environment thought hydrogen bonding network. In order to explore this, far-UV CD spectroscopy was employed to measure the binding event of phosphite dianion. As shown in Fig 44, the binding of the phosphite dianion indeed alters the active site micro-environment and slightly influences the protonic and tautomeric states of the sixmembered ring aminopyrimidine. The AP form blue-shifts 2-3 nm with decreased amplitude and the same holds true for the IP form, indicating that the equilibrium shifts towards the conjugate acid $\mathrm{APH}^{+}$form. Identical equilibrium shifts but with considerable intensity were observed for the physiological substrate F6P as well as the non-phosphorylated sugars fructose and xylulose, suggesting that the chemical shift towards the $\mathrm{APH}^{+}$form is a prerequisite for the nucleophilic reaction (Fig 66). In other perspective, the binding of phosphite dianion activates the system by shifting the equilibrium as the substrates do, so this could partially explain the 2.5-fold acceleration for the velocity of DHEThDP intermediate formation detected by the stopped-flow analysis. In addition, CD spectra with increased phosphite dianion concentration (up to 50-100 $\mathrm{mM}$ ) results in chemical equilibrium with the same amplitude, which supports our previous stopped-flow studies with a series of phosphite dianion concentration. Another obvious feature lies between 270-280 nm where a drop of intensity was observed. The same spectral characteristic is observable both in human and E.coli $\mathrm{TK}$ in a variety of $\mathrm{CD}$ measurements including the $\mathrm{pH}$-titration experiments discussed in the LBHB chapter. Typically the aromatic amino acid residues are responsible for the CD absorption in this wavelength range, it is therefore questionable whether the signal drop at 270-280 nm arises from the aromatic side chains or from other unknown chemical processes. In the study of OMPDC, another four oxydianions including phosphate were tested for the decarboxylation of the truncated substrates (Reyes et al., 2015), so we tested the binding of phosphate by CD spectrometer. Due to that calcium and phosphate ion immediately form calcium phosphate which is insoluble in aqueous solution, the $5 \mathrm{mM} \mathrm{CaCl}_{2}$ has to be removed from the measuring buffer. The obtained CD spectra shows similar characteristics as that of the phosphite, which strongly suggests similar binding mode of these two oxydianions. 


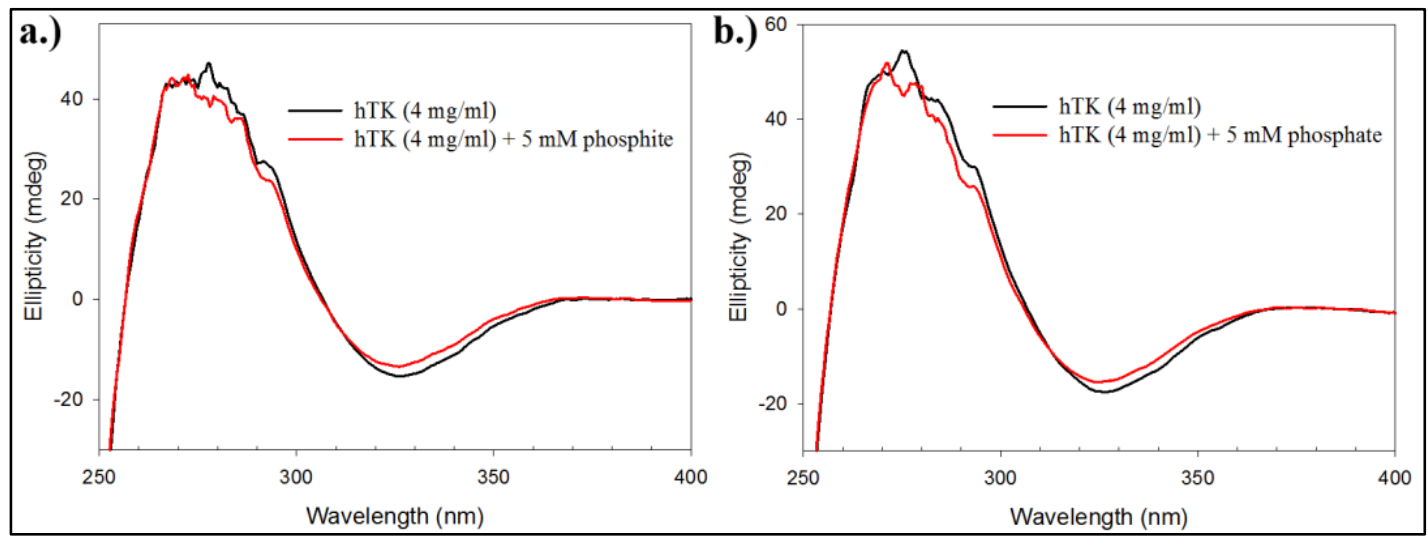

Fig 44: Circular Dichroism (CD) measurement for the binding of phosphite and phosphate dianions to the active site of $\boldsymbol{h T K}$. a.) Phosphite; b.) Phosphate. Normalization of protein concentrations were performed for both spectra.

In order to visualize the binding mode of phosphite dianion to $h \mathrm{TK}$ and explore possible reasons for the reaction acceleration form structural perspective, we have determined the crystal structure of $h \mathrm{TK}$ in complexed with phosphite dianion at a resolution of $1.5 \AA$ and refined to reasonable statistics $\left(\mathrm{R}_{\text {work }}=15.04 \%, \mathrm{R}_{\text {free }}=17.53 \%\right)$, the occupancy of phosphite dianion could also be calculated to $95 \%$. The additional positive difference electron density map found at the entrance of the active site which is around $9.5 \AA$ away from the $\mathrm{C} 2$ of ThDP was interpreted and modelled as phosphite dianion (Fig 45). The phosphate atom points to the reacting center with the three oxygen atoms facing to the entrance of the active site. Further comparison with the F6P-ThDP structure indicates that the phosphite dianion processes similar binding position as the phosphate moiety of F6P. 


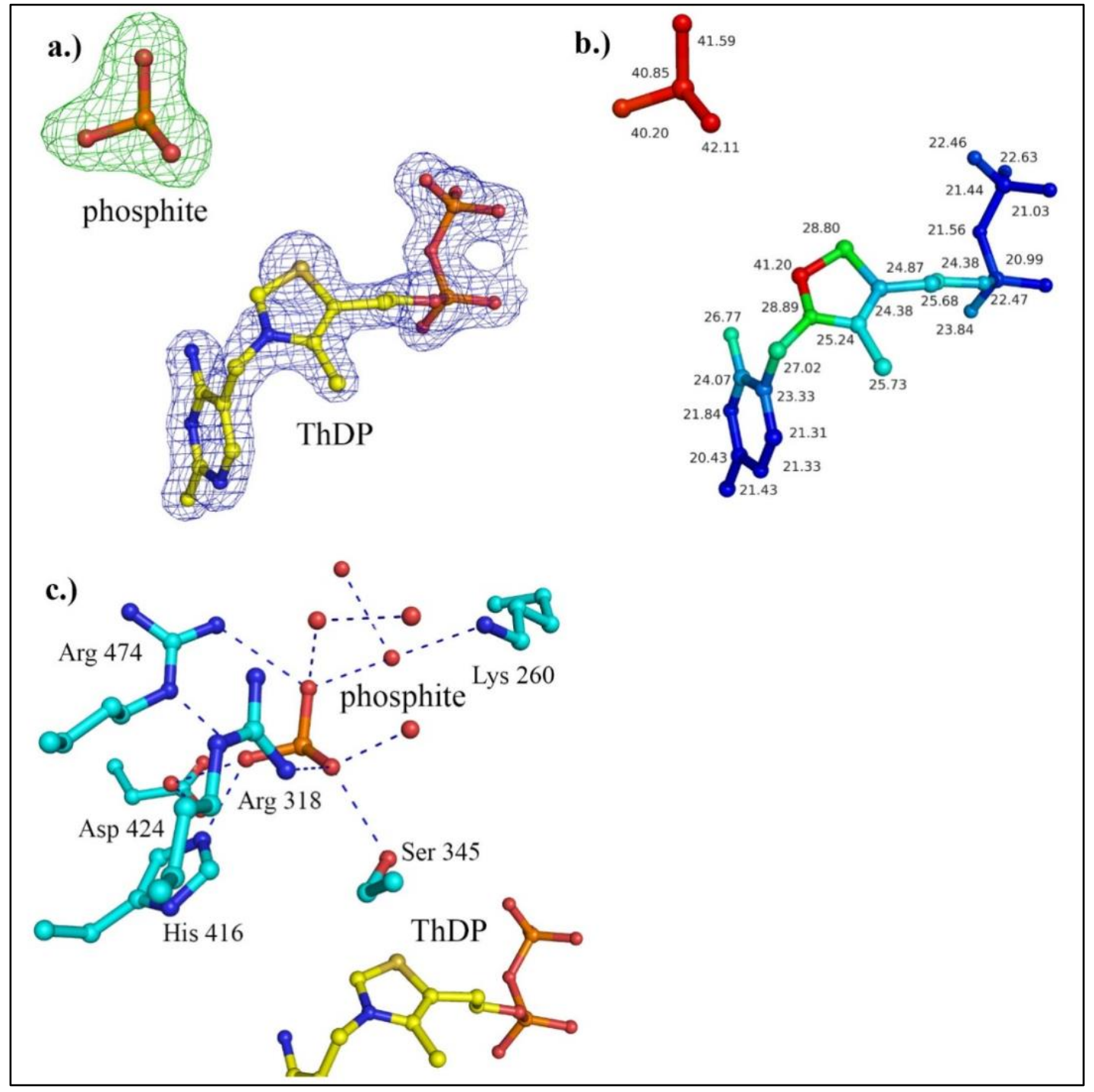

Fig 45: Crystal structure of $\boldsymbol{h T K}$ with phosphite dianion binding at the active site. The crystal structure of phosphite dianion binding to the active site of $h$ TK was determined at a resolution of $1.5 \AA$ by soaking obtained crystals with $100 \mathrm{mM}$ phosphite dianion dissolved in pH adjusted cryo-solution. a.) Phosphite dianion shown with a residue-omitted Fo-Fc map (contoured at $3 \sigma$ level, green) bound approx. $9 \AA$ away from the cofactor ThDP which is surrounded by a 2 Fo-Fc map (contoured at $1 \sigma$ level, blue). b.) Atoms of phosphite dianion and ThDP are colour-labelled according to their individual Bfactors with most mobile atoms in red $\left(\approx 40 \AA^{2}\right)$ and less mobile ones in blue $\left(20-23 \AA^{2}\right)$. c.) Interactions of the phosphite dianion with the active site residues (shown in cyan) partially mediated by water molecules (shown as red spheres) are indicated by blue dashed lines.

Several amino acid residues as well as 5-6 water molecules are responsible for the binding of the phosphite dianion at the active site. Detailed analysis of the binding mode for the ligand reveals that each of the oxygen atoms is coordinated by three hydrogen bonds either with the side chain or main chain of Arg474, Arg318, His416 and Ser345, or though water molecules. 
Notably, the side chains Asp424 and Lys260 form hydrogen bonding interactions with the phosphite dianion with the help of two additional water molecules. Despite the relatively strong hydrogen bonding interactions, the atomic B-factors of the phosphite dianion are 1.5-2 fold higher compared with that of the cofactor ThDP and 1.2-1.5 fold higher than the surrounding active sites residues, which clearly suggests a higher flexibility of the bound phosphite dianion. Strikingly, the atomic B-factor on the reactive $\mathrm{C} 2$ atom, which is the nucleophile that attacks the carbonyl carbon of different substrates, suggests a nearly 2-fold increased B-factor relative to the rest part of ThDP. This increased flexibility of the carbon atom on the $\mathrm{C} 2$ position is further supported by the diffused electron density in the $2 \mathrm{Fo}-\mathrm{Fc}$ map contoured at $1 \sigma$ level. The resting state structure of $h \mathrm{TK}$ at a slightly lower resolution

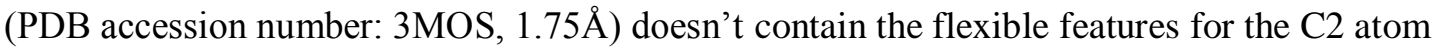
and the whole cofactor ThDP shows lower atomic B-factors compared with the structure in complex with phosphite dianion. These structural characteristics indicate that the binding of exogenous phosphite dianion induces the $\mathrm{C} 2$ atom of $\mathrm{ThDP}$ as well as cofactor itself into a hyper-reactive state which is poised for the nucleophilic attack on the carbonyl function of substrates.

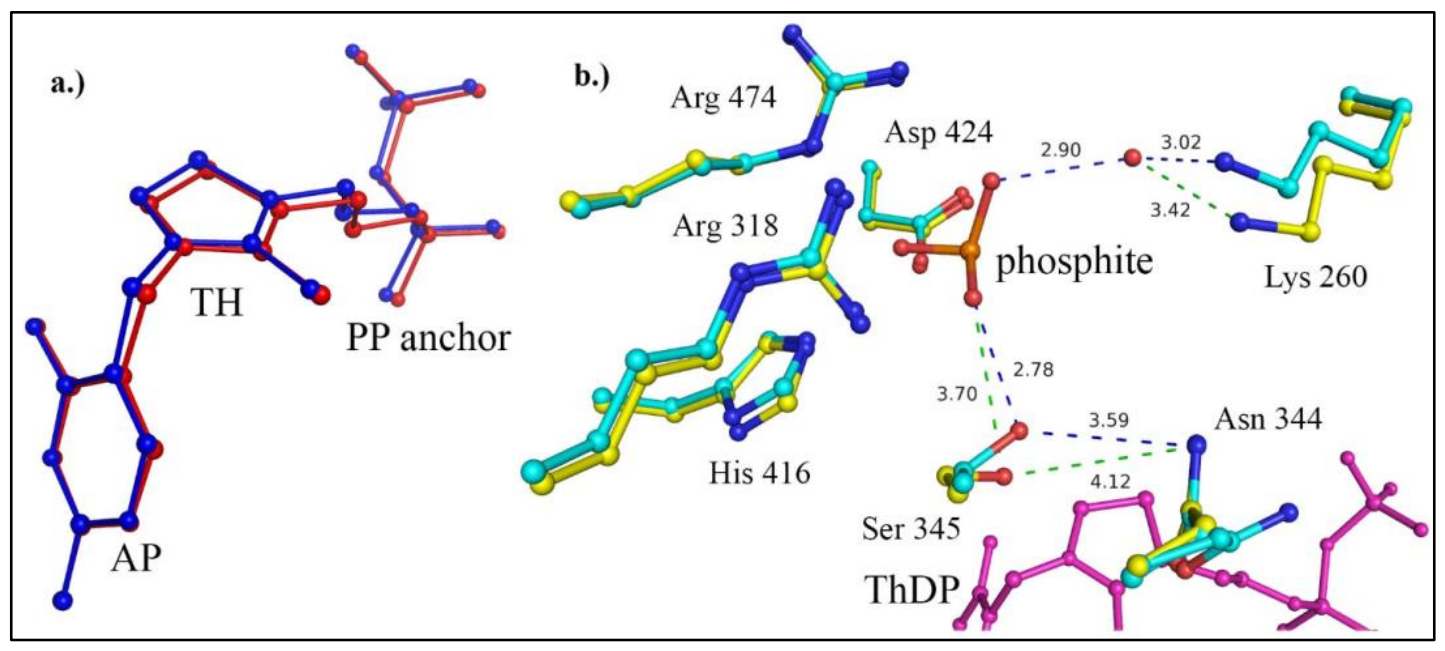

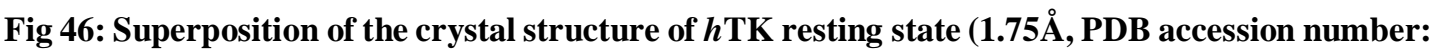
3MOS) with $\boldsymbol{h T K}$ in complex with phosphite dianion (1.5 $\AA$ ). a.) Superposition of the cofactor ThDP shown in ball-stick representation (structure of resting state in red and structure in complex with phosphite in blue). The aminopyrimidine (AP), thiazolium ring (TH) and the diphosphate (PP) anchor are highlighted. b.) Superposition of the binding pocket for phosphite dianion within the active site of $h \mathrm{TK}$ (in cyan) with that of the resting state structure (in yellow). Selected hydrogen bonding interaction are highlighted in dashed line (blue for phosphite structure, green for RS) with labelled inter-atomic distances. The cofactor ThDP from resting state structure is shown in magenta for positional comparison. A water molecule linking the phosphite dianion with a lysine residue is shown in red sphere. 
More interestingly, when the ThDP cofactors from resting state structure and the structure in complex with phosphite dianion are superimposed, the six-membered AP ring especially the $\mathrm{C} 2$ '-methyl group are perfectly matched but the TH ring as well as the pyrophosphate anchor slightly shifts towards the reacting center. This minor spatial movement might facilitate the encounter of the non-physiological substrate with the reactive $\mathrm{C} 2$ atom thus increase the efficiency of catalysis. Superposition of the binding pocket for the phosphite dianion reveals similar architecture, Arg474, His416, Arg318 and Asp424 which are at one side of the active site's entrance are well superimposable while Ser345 and Lys260 show subtly different orientations. Upon phosphite binding Ser345 is attracted by the oxygen of the dianion and forms a hydrogen bond to the ligand with normal HB distance (2.78 $\mathrm{A})$, but in the resting state structure the orientation of Ser345 is slightly downward with a distance of $3.70 \AA$ if the phosphite dianion were bound. This minor spatial change could slightly open the tunnel for the substrate therefore enhancing the reaction velocity. Another observable structural change is the Lys260 interacting with the phosphite dianion though a water molecule. Asn344 has two alternative conformations in the resting state but only shows one conformation in the structure in complex with phosphite dianion.

\subsection{Dynamic hotspots in human and E.coli transketolases}

\subsubsection{Flipping histidine observed in E.coli transketolase}

Ring flipping of the histidine side chain has been proposed in E.coli transketolase when the structure of EcTK in complex with substrate was determined to sub-angstrom resolution. Careful analysis of anisotropic displacement parameters (ADPs) revealed that His473 could flip over $180^{\circ}$ and adopts two alternative conformations (Neumann and Tittmann, 2014). In order to explore the catalytic function of this histidine flipping, previous colleagues had performed a comprehensive study of His473A mutant and the results revealed an inferior role of His473 for the binding and alignment of acceptor substrate (Lüdtke, 2012). However, the catalytic function of the ring flipping is still unclear and needs further studies. Further analysis of the crystal structure revealed that the second conformation of His 473 would make hydrogen bond contact with Asp469 via a water molecule, therefore D469N/A as well as H473N mutants were generated and studied in this thesis.

\subsubsection{Observation of histidine flipping in the crystal structure of $E c$ TK D469N and $E c$ TK D469A}

The crystal structures of EcTK D469N and D469A have been determined to $1.06 \AA$ ( $\mathrm{R}_{\text {work }}=$ $\left.11.24 \%, \mathrm{R}_{\text {free }}=12.57 \%\right)$ and $1.15 \AA\left(\mathrm{R}_{\text {work }}=13.35 \%, \mathrm{R}_{\text {free }}=15.26 \%\right)$ respectively. Shown 
in Fig 47, the calculated electron density ( $2 \mathrm{mFo}-\mathrm{DFc}$ map contoured at $2 \sigma$ level) around His473 in both structures are not well defined compared with these of the neighboring His residues at the active site, such as His100 and His66. The electron density for the $\mathrm{C} \varepsilon$ and $\mathrm{N} \varepsilon$ atoms is not clearly separated as it should be, but rather diffuse along the $\mathrm{C} \varepsilon-\mathrm{N} \varepsilon$ bond. Careful analysis of the thermal motions (ADPs) of these two atoms in EcTK D469N crystal structure reveals that $\mathrm{C} \varepsilon$ and $\mathrm{N} \varepsilon$ would move along their bond axis. This anomalous behavior is very unlikely because it violates the rigid bond rule, but this could be better explained in a way that His473 undergoes imidazole ring flipping and adopts two alternative conformations. The difference between the two conformations is that either $\mathrm{N} \varepsilon$ or $\mathrm{C} \varepsilon$ form hydrogen bonding with the central water molecule then interact with cofactor and other active site residues. Carbonbound protons can only act as H-bond donors while nitrogen-bound protons can act as both donors and acceptors, which might play a role in catalysis but is still not clear yet. The water molecule connecting His473 and Asn469 in the D469N structure is very flexible based on the observation of extra electron density, while that water in the D469A structure tenders to be more rigid. Anisotropic B-factors for the D469A structure is slighter higher but ADPs for Ce and $\mathrm{N} \varepsilon$ reveal same ring flipping behavior. Therefore, mutation of Asp469 to Asn and Ala doesn't affect the flipping of His473.

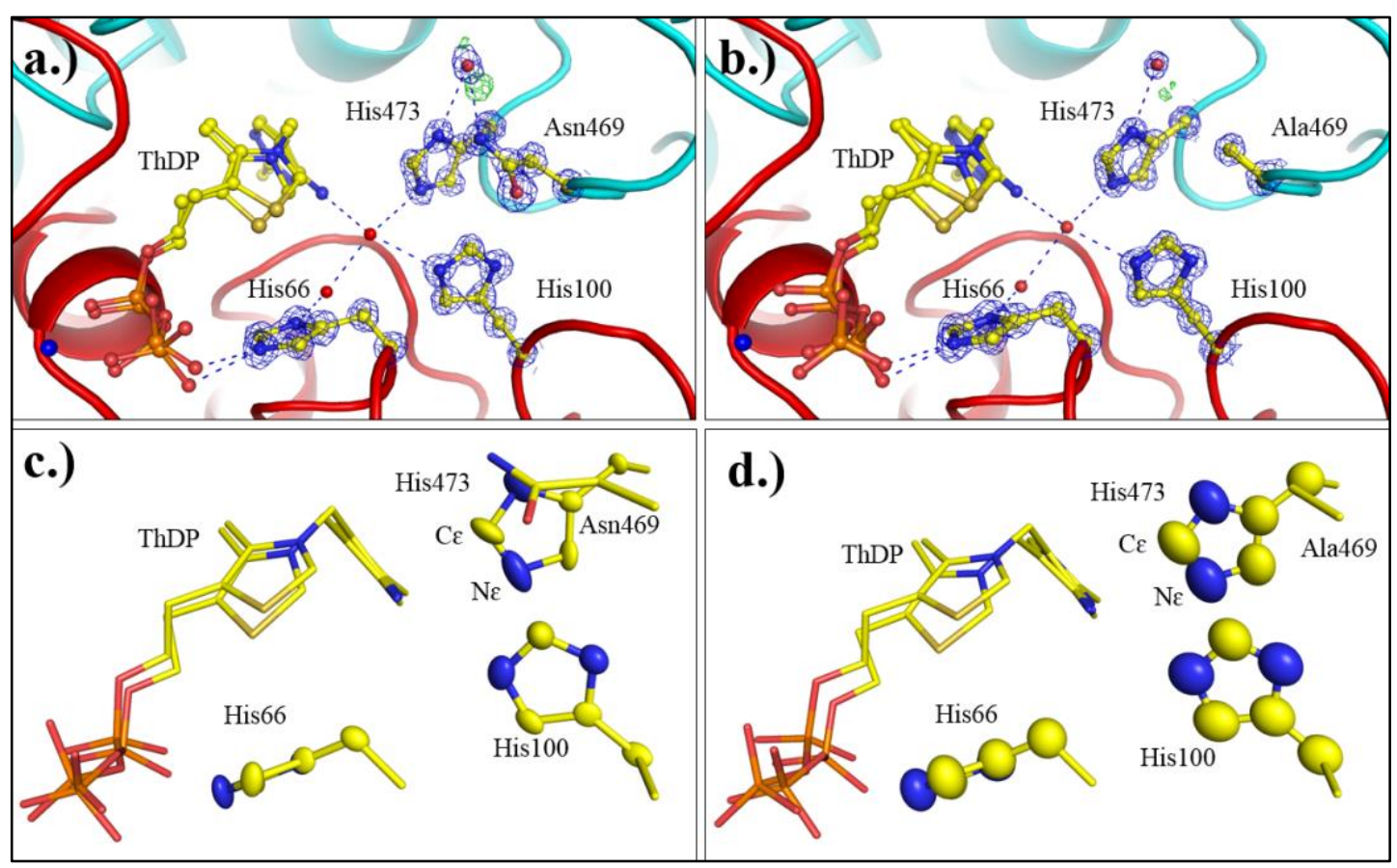

Fig 47: Detailed view of the "flipping histidine" in the active site of EcTK D469N and D469A mutants. a.) Active site architecture of EcTK D469N at a resolution of $1.06 \AA$. Amino acid residues His66, His100, His473 and Asn469 are shown in ball-stick representation surrounded by a $2 \mathrm{mFo-DFc}$ map (contoured at $2 \sigma$ level, blue). Please note that the electron density for His 473 is more diffuse while 
others (His66 and His100) are well-defined. Water molecules are shown as red spheres and the water

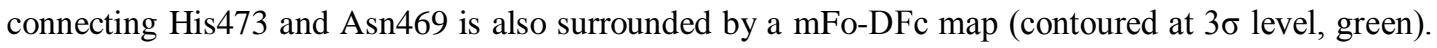
Hydrogen bonding interactions are shown as blue dashed lines and the two monomers of EcTK are shown as cartoon representation in red and cyan respectively. b.) Active site architecture of EcTK

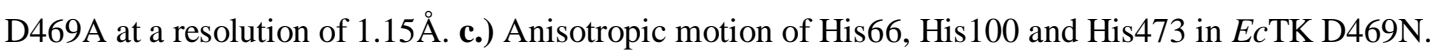
The atomic displacement parameters (ADPs) of His473 indicate that $\mathrm{N} \varepsilon$ and $\mathrm{C} \varepsilon$ would thermally move along their bond axis, which is not possible because of the rigid bond rule. This suggests that His473 exists in two conformations though ring flipping. d.) Anisotropic motion of His66, His 100 and His473 in EcTK D469A. Although atomic B-factors are higher but still the same ring flipping behaviour could be observed.

\subsubsection{Preliminary studies of "histidine flipping"-related active site mutants}

In order to study the impact of Asp469 on the tautomeric and protonic states of the cofactor ThDP, EcTK D469N and D469A variants were first measured by a Circular Dichroism (CD) based $\mathrm{pH}$ titration experiment in the far UV-range. Since EcTK is purified as apo-enzyme, additional cofactor ThDP needs to be supplemented to reconstitute the holo-enzyme. $100 \mu \mathrm{M}$ and $300 \mu \mathrm{M}$ were used for the regeneration of D469A and D469N holo-enzyme respectively. The spectra reveals that using of $300 \mu \mathrm{M}$ ThDP would produce very noisy curve at the near UV range and influences the IP signal around $295 \mathrm{~nm}$ while $100 \mu \mathrm{M}$ ThDP leads to clean spectra. This is because ThDP itself would absorb in the near UV range. Therefore, it gives guide to further work on $E c$ TK that concentration of cofactor used for reconstitution needs to be determined first before performing other experiments. The AP form (around $320 \mathrm{~nm}$ ) increases with $\mathrm{pH}$ values and reaches maximal amplitude at around $\mathrm{pH}$ 7.0. Plotting of the intensity of the AP form against the corresponding $\mathrm{pH}$ value and fitting the data points according to equation 5a for single ionizing group give $\mathrm{pKa}$ values of $6.11 \pm 0.26$ for D469A and $6.38 \pm 0.20$ for $\mathrm{D} 469 \mathrm{~N}$. These values as well as spectra itself deviates from that of the wild type EcTK (unpublished data). 


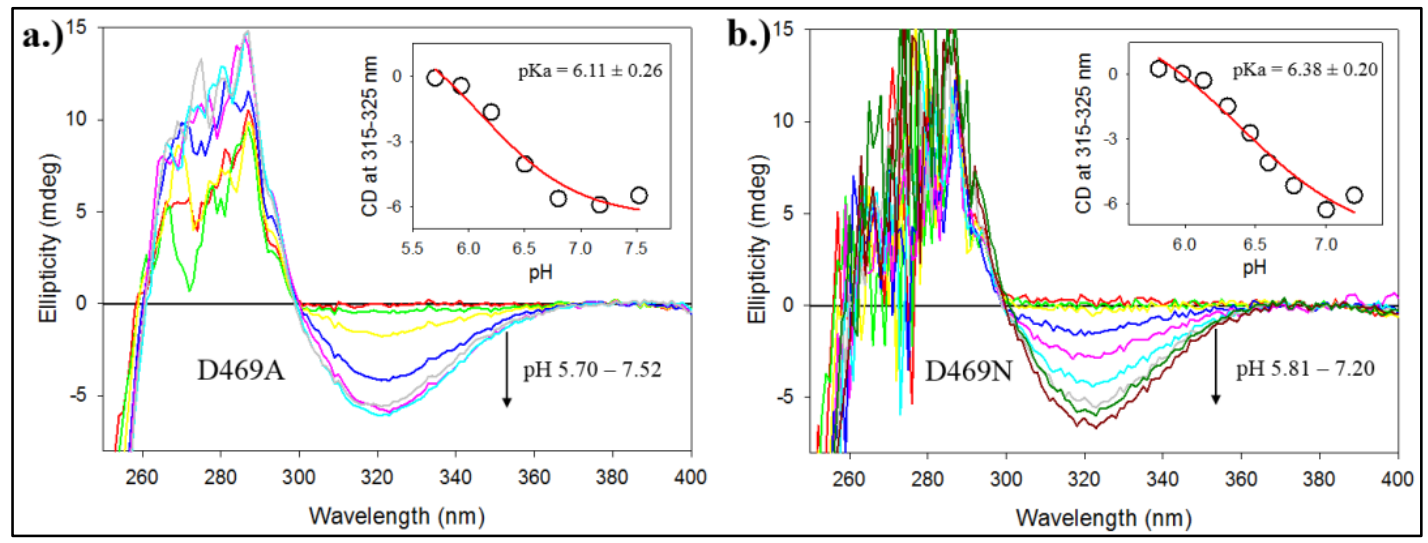

Fig 48: Near-UV CD spectra of the tautomeric and protonic states of ThDP at different pH values for $\boldsymbol{E} \boldsymbol{c}$ TK D469N and D469A. Samples containing $1.5 \mathrm{mg} / \mathrm{ml}$ enzyme in $20 \mathrm{mM}$ MES, $20 \mathrm{mM}$ glycylglycine were titrated by addition of Tris base $(1 \mathrm{M}, \mathrm{pH} 11)$ and the $\mathrm{pH}$ values were measured using a Minitrode $\mathrm{pH}$ electrode (Hamilton). The measurements were performed at $20^{\circ} \mathrm{C}$ with a path length of $10 \mathrm{~mm}$ and recorded between $250-400 \mathrm{~nm}$. a.) Near-UV CD spectra for the $\mathrm{pH}$ titration experiment on EcTK D469A. b.) Near-UV CD spectra for the pH titration experiment on EcTK D469N. Inset: pH dependence of AP formation at 325-335 nm (average value). The $\mathrm{pKa}$ was determined using equation 5a.

Single-mixing stopped-flow analysis of the pre-steady-state kinetics with physiological substrate F6P have revealed a very slow process of substrate binding $(20 \mathrm{mM}$ F6P with reaction time up to $1000 \mathrm{~s}$, Fig 68) for both D469N and D469A variants. However, when the artificial substrate HPA was used and the DHEThDP intermediate was measured at $300 \mathrm{~nm}$, both variants exhibit similar rate constants as the wild type for the formation of the DHEThDP intermediate. Longer lifetime of the intermediate was observed on the D469A variant compared with wild type and D469N. The interesting point of these results is that both D469N and D469A mutants could use artificial substrate HPA but bind the native substrate F6P in a very slow manner.
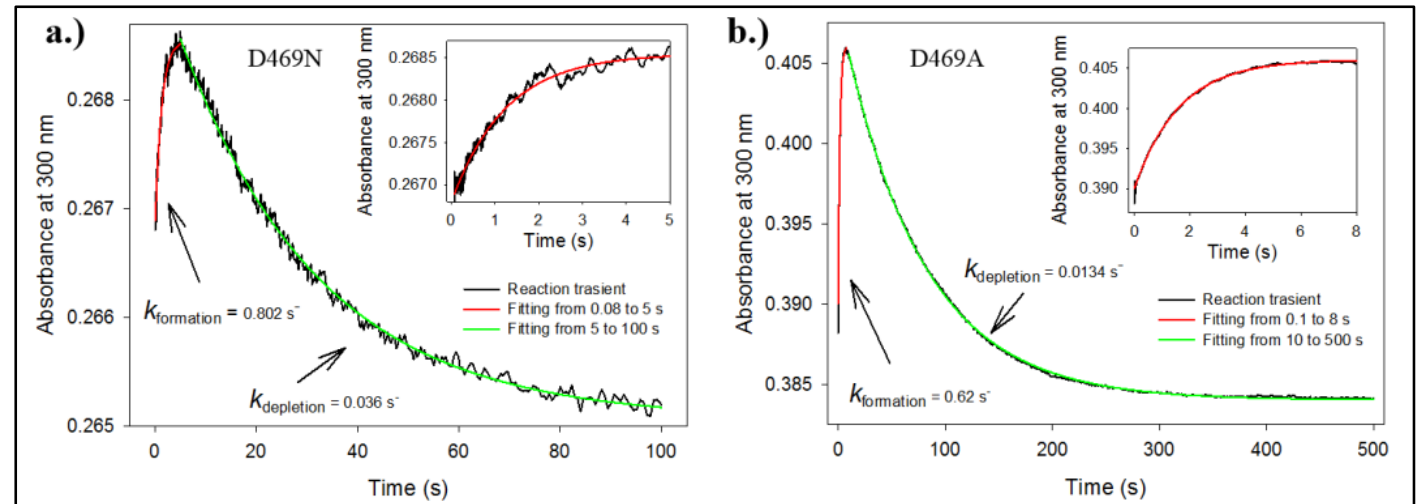

Fig 49: Single turn-over reaction of $E c$ TK D469N and D469A with donor substrate $\beta$ hydroxypyruvate (HPA). Reaction of $4 \mathrm{mg} / \mathrm{ml} \mathrm{EcTK}(55.5 \mu \mathrm{M}$ active sites) with HPA (equimolar 
concentration) in $0.3 \mathrm{mM}$ ThDP, $5 \mathrm{mM} \mathrm{CaCl}_{2}, 50 \mathrm{mM}$ glycylglycine (pH 7.6) measured at $25{ }^{\circ} \mathrm{C}$. Reactions were initiated by 1:1 mixing and recorded by stopped-flow spectrometer at $300 \mathrm{~nm}$ (path length $10 \mathrm{~mm}$ ). Rate constants for the formation and depletion of the DHEThDP intermediate were determined according to a single exponential equation $\left(\mathrm{A}=\mathrm{A} 1 * \mathrm{e}^{-k 1 * \mathrm{t}}+\right.$ offset $)$. a. $)$ Reaction transient of $E c$ TK D469N. b.) Reaction transient of EcTK D469A. Inserts: Initial phase for the formation of DHEThDP intermediate.

Further pre-steady-state kinetics studies were focused on the EcTK H473N variant. Singlemixing stopped-flow experiments with F6P and HPA reveal that EcTK H473N could neither bind physiological substrates nor artificial substrates. Crystallization of this variant unfortunately didn't work out, making the structural analysis very difficult. In order to study the cofactor binding, a series of ThDP concentration were mixed with apo EcTK and the reconstitution velocity were measured by a stopped-flow spectrometer and plotted against the utilized cofactor concentration. Data points of the wild type reveal a linear dependency until $15 \mathrm{mM}$ cofactor, suggesting that the cofactor binding of EcTK-wt is a diffusion-controlled manner. H473N exhibits different behavior with a hyperbolic dependency of the reconstitution.
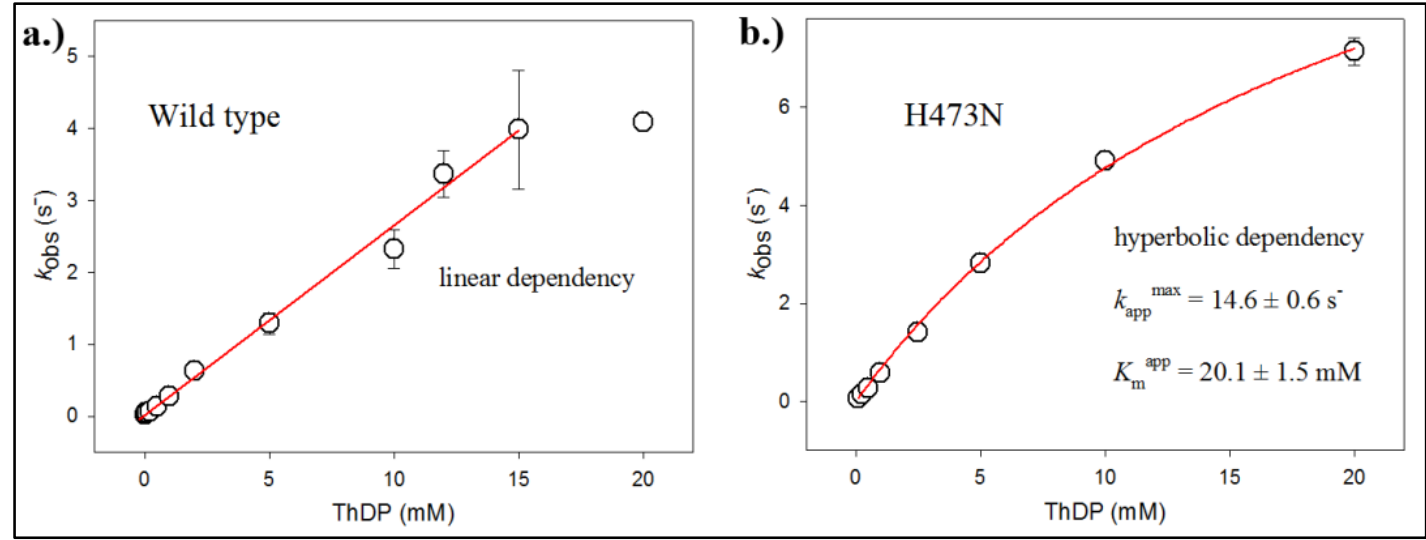

Fig 50: Reconstitution of holo-EcTK from cofactor ThDP binding to apoenzyme. Typical reactions were performed by 1:1 mixing of $4 \mathrm{mg} / \mathrm{ml}$ apo- $E c$ TK with increasing concentration of ThDP in $5 \mathrm{mM}$ $\mathrm{CaCl}_{2}, 50 \mathrm{mM}$ glycylglycine (pH 7.6) and measured by a UV-Vis spectrometer at $320 \mathrm{~nm}$ at $25^{\circ} \mathrm{C}$. Reaction constants were determined according to a single exponential equation $\left(\mathrm{A}=\mathrm{A} 1 * \mathrm{e}^{-k l^{*}}+\mathrm{offset}\right)$. a.) ThDP binding of EcTK wild type. The velocity for the reconstitution of holoenzyme shows a linear dependency on the utilized ThDP concentration. b.) ThDP binding of EcTK H473N. The velocity for the reconstitution of holoenzyme shows a hyperbolic dependency on the utilized ThDP concentration. 


\subsubsection{Catalytic function study of the intrinsic dynamics in human transketolase}

Current study of enzyme catalysis have been largely focused on the kinetics and thermodynamics of the enzyme-catalysed conversion from substrates to products, or on the static three dimensional structures of catalytic snapshots during enzymatic reaction (Eisenmesser et al., 2005). However, little is known about the correlation between the dynamic catalytic residues and the enzymatic reaction efficiency. Previous studies of human TK in complex with the covalent substrate-ThDP conjugates have revealed a structural plasticity of the active sites including intermediates, backbone carbonyl of Gly123, side chains of Gln 428 and Ser427 and water molecules (Fig 51). A hydrogen bonding communication pathway between substrate $\rightarrow$ water $\rightarrow$ backbone (Gly123) $\rightarrow$ water $\rightarrow$ Ser427 $\rightarrow$ Gln $428 \rightarrow$ substrate was observed and assumed to play catalytic role during the enzymatic reaction.

\subsubsection{Crystal structures of $h \mathrm{TK}$ S427A, Q428A and H77A reveal different active site architectures}

In order to study the function of this structural plasticity, three single-site enzyme variants S427A, Q428A and G123A were generated and studied structurally and kinetically. The crystal structure of human TK S427A in complex with donor substrate F6P was determined at $1.25 \AA\left(\mathrm{R}_{\text {work }}=13.46 \%, \mathrm{R}_{\text {free }}=16.13 \%\right)$. Interestingly, in this structure side chain of Gln428 and backbone of Gly123 were only observed in one conformation while in wild type Gln428, S427 and backbone of Gly123 adopt two alternative conformations. Three water molecules were observed to communicate the Gln428 and the backbone of Thr122, which in wild type is mediated by the second conformation of Ser427 and backbone of Gly123. We have also determined the crystal structure of human TK Q428A in complex with F6P at medium resolution $\left(1.5 \AA, R_{\text {work }}=16.87 \%, R_{\text {free }}=19.24 \%\right)$. The structure reveals different active site architecture compared with wild type and S427A. The backbone of Gly123 adopts two conformations and the second conformation interacts though two water molecules with Ser427 which only exhibits one conformation. In all above mentioned structures, the backbone of the first conformation of Gly123 interacts with the substrate's C1-OH group. Although His77 doesn't participate in this hydrogen bonding wire, it interacts with the $\beta$-phosphate group of the cofactor and plays a role in the binding of ThDP. The structure of human TK H77A was determined at a relatively low resolution $\left(1.9 \AA, \mathrm{R}_{\text {work }}=17.36 \%, \mathrm{R}_{\text {free }}=22.68 \%\right)$ in complex with F6P. The active site architecture of H77A reveals that Gln428, Ser427 and the backbone of Gly123 show only one conformation due to the rearrangement of water positions near the mutation. Three water molecules mediates the interaction of the $\beta$-phosphate group of ThDP and the backbone of Thr122 and Gly123, with the middle water forming hydrogen bonding with $\mathrm{C} 1-\mathrm{OH}$ group of the substrate. It is possible that the absence of alternative conformations 
is artificial because of low resolution crystal data therefore higher resolution structure of this mutant is needed in the future.

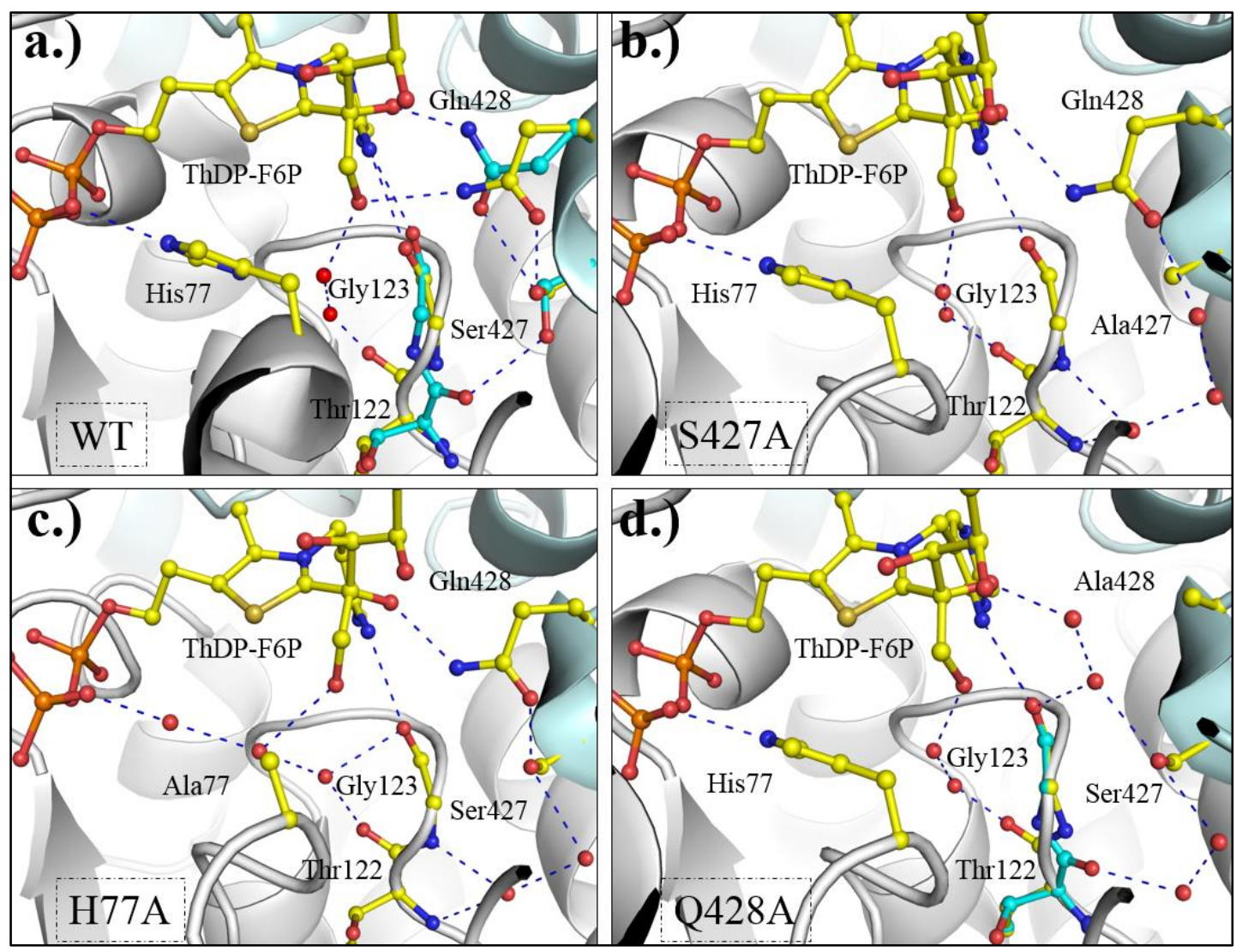

Fig 51: Detailed view of the "dynamic hotspots" in human TK and related active site mutations.

a.) Active site architecture of wild type with covalently bound donor substrate F6P. Side chains of Ser427 and Gln428 and the main chains of Thr122 and Gly123 adopt two alternative conformations shown in yellow and cyan respectively. b.) Active site of mutant S427A with covalently bound donor substrate F6P. No alternative conformations were observed. c.) Active site of mutant H77A with covalently bound donor substrate F6P. No alternative conformations were observed. d.) Active site of mutant Q428A with covalently bound donor substrate F6P. Alternative conformations were observed only for the main chains of Thr122 and Gly123. For all figures, the two monomers of human TK are shown in cartoon representation in grey and cyan respectively. Related water molecules are shown as red spheres and the hydrogen bonding interaction are highlighted as blue dashed lines, interatomic distances are not shown for the reason of clarity.

\subsubsection{Preliminary functional studies of the "dynamic hotspots" in human TK}

X-ray crystallographic results of the "dynamic hotspots"-related human TK variants have revealed structural features of Gln428, Ser427 and His77. In order to explore the function of 
these amino acid residues in the donor half reaction, single-mixing stopped-flow experiments were performed using native substrate F6P. Reaction conditions were similar to that of the LBHB but measured at $20{ }^{\circ} \mathrm{C}$ instead of $4{ }^{\circ} \mathrm{C}$. For the wild type, the velocity of AP form depletion shows a hyperbolic dependency against the utilized F6P concentration. The reaction can also be interpreted as a two-step reaction similar to the LBHB chapter with a $k_{\mathrm{obs}}{ }^{\max }$ around $79.14 \pm 4.60 \mathrm{~s}^{-}$and $K_{\mathrm{s}}^{\text {app }}$ of $1.16 \pm 0.32 \mathrm{mM}$. The reverse reaction of the covalent ThDP-F6P formation is around $11.59 \pm 4.59 \mathrm{~s}^{-}$.

The donor half reaction of H77A variant shows largely impaired reactivity with $k_{\mathrm{obs}}{ }^{\max }$ around $2.15 \pm 0.30 \mathrm{~s}^{-}$which is only $3-4 \%$ of that of the wild type. The substrate affinity also decreases about 3 -fold $\left(K_{\mathrm{s}}^{\text {app }}=3.04 \pm 1.68 \mathrm{mM}\right)$ compared with wild type. Taking into the structural results, we could propose that the two dynamic conformations of Gln428, Ser427, and backbone of Gly123 are very important for the ThDP-F6P intermediate formation. The absence of the alternative conformations caused by mutation of His77 to Ala makes the enzyme almost dead. The Q428A variant shows $40 \%$ activity of wild type for the donor half reaction but exhibits much higher substrate affinity $\left(K_{\mathrm{s}}^{\text {app }}=0.17 \pm 0.04 \mathrm{mM}, 5\right.$-fold to wild type). Interestingly, the second phase for donor half reaction of Q428A also shows hyperbolic dependency with a $k_{\mathrm{obs}}{ }^{\max }$ around $14.21 \pm 0.77 \mathrm{~s}^{-}$and $K_{\mathrm{s}}{ }^{\text {app }}$ of $1.54 \pm 0.28 \mathrm{mM}$. According to previous studies (Lüdtke, 2012), the two apparent phases reflect the non-equivalence property of the two active sites of transketolase, or in general ThDP enzyme family. The reaction happening at the "active" active site is faster than that of the "dormant" active site (the second phase for the wild type and other mutants are scattered therefore are not discussed here). Although the velocity of donor half reaction of mutants S427A and G123A are apparently slower than the wild type, the data points are scattered, making quantitative analysis difficult. Therefore further measurements need to be performed, for example, at $4{ }^{\circ} \mathrm{C}$. 


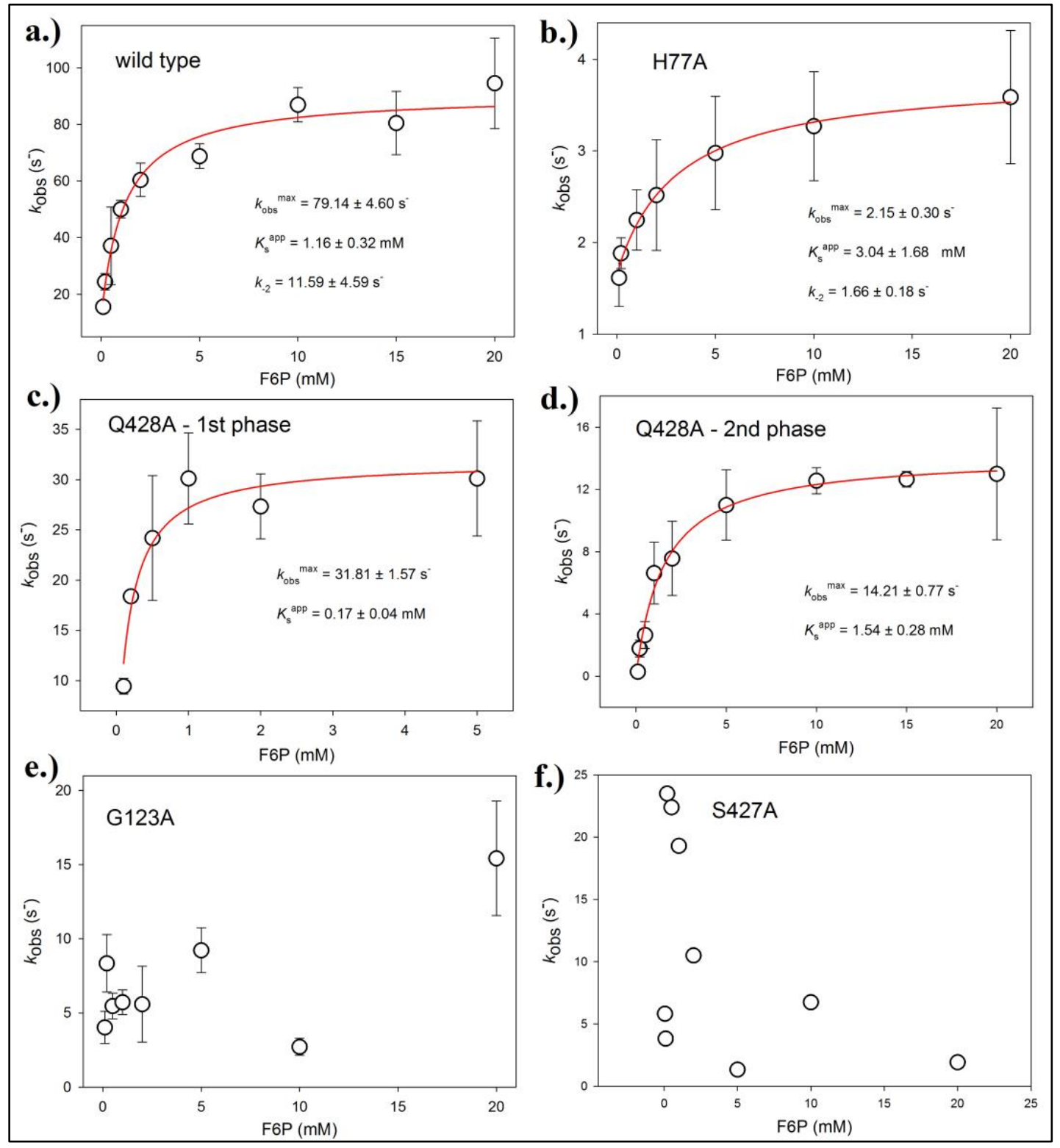

Fig 52: Stopped-flow analysis of the pre-steady-state kinetic of the $h \mathrm{TK}$ donor half reaction. Typical reactions were performed by mixing $4 \mathrm{mg} / \mathrm{ml} h \mathrm{TK}$ with increasing concentration of fructose 6phosphate (F6P) in $5 \mathrm{mM} \mathrm{CaCl}_{2}, 50 \mathrm{mM}$ glycylglycine (pH 7.6) and measured by UV-Vis spectrometer at $325 \mathrm{~nm}$ at $20^{\circ} \mathrm{C}$. Reaction constants were determined according to a double exponential equation (A $=\mathrm{A} 1 * \mathrm{e}^{-k l^{*} \mathrm{t}}+\mathrm{A} 2 * \mathrm{e}^{-k 2 * t}+$ offset). a.) First phase of $h \mathrm{TK} \mathrm{WT}$; b.) First phase of $h \mathrm{TK}$ H77A. c.) First phase of $h \mathrm{TK} \mathrm{Q} 428 \mathrm{~A}$; d.) Second phase of $h \mathrm{TK} \mathrm{Q} 428 \mathrm{~A}$; e.) First phase of $h \mathrm{TK}$ G123A; f.) First phase of $h \mathrm{TK}$ S427A. 
In order to study the impact of the "dynamic hotspots" on the tautomeric and protonic states of ThDP, $\mathrm{pH}$ titration experiments were performed according to the chapter of LBHB and measured by Circular Dichroism (CD) spectroscopy in the far-UV range. The spectra of Q428A, S427A and H77A exhibit similar properties with AP form (negative signal at 320-330 $\mathrm{nm}$ ) and IP form (positive signal at 290-300 $\mathrm{nm}$ ) increasing with $\mathrm{pH}$ values. The third tautomeric form $\mathrm{APH}^{+}$, which could not be clearly identified by $\mathrm{CD}$ spectroscopy, decreases with $\mathrm{pH}$ values due to $([\mathrm{AP}]+[\mathrm{IP}]) /\left[\mathrm{APH}^{+}\right]$equilibrium. The $\mathrm{pKa}$ values for $\mathrm{Q} 428 \mathrm{~A}$ and S427A were determined to $6.54 \pm 0.15$ and $6.45 \pm 0.13$ respectively which are very similar to that of the wild type enzyme $(5.96 \pm 0.07)$, suggesting that these amino acid residues are not involved in the process of $([\mathrm{AP}]+[\mathrm{IP}]) /\left[\mathrm{APH}^{+}\right]$equilibrium. Although $\mathrm{pKa}$ of $\mathrm{H} 77 \mathrm{~A}$ was not successfully determined due to data noisy, the similarity of the CD spectra might suggest an identical $\mathrm{pKa}$. Therefore, His 77 doesn't participate in the tautomeric process either. The stability of mutant G123A has been dramatically deceased compared with wild type and other variants, which leads to unsuccessful crystallization and noisy CD spectra. 

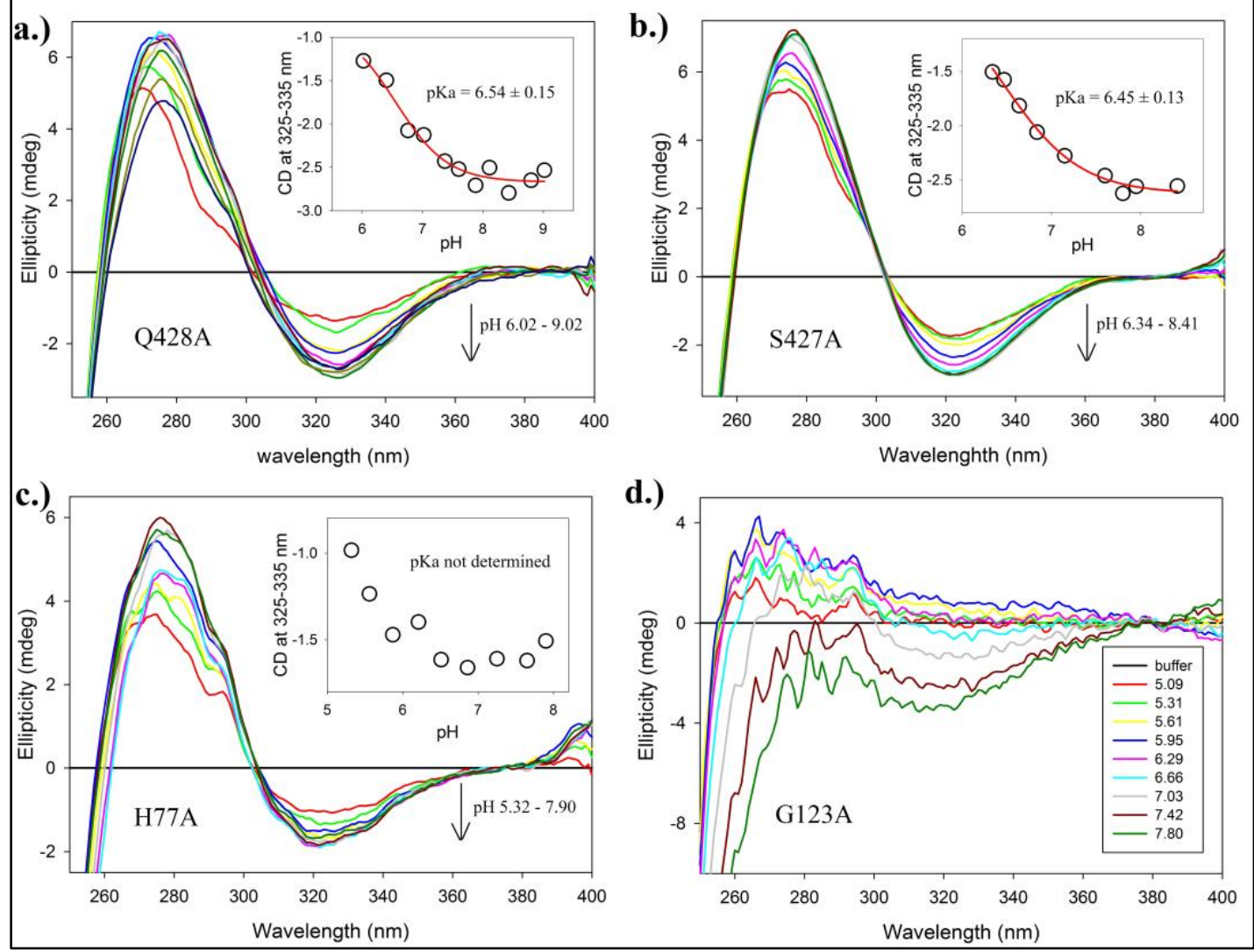

Fig 53: Near-UV CD spectra of the tautomeric and protonic states of ThDP at different pH values for $\boldsymbol{h T K}$ “dynamic hotspots"-related active site variants. Samples containing $1.5 \mathrm{mg} / \mathrm{ml}$ enzyme in $20 \mathrm{mM}$ MES, $20 \mathrm{mM}$ glycylglycine were titrated by addition of Tris base $(1 \mathrm{M}, \mathrm{pH} 11)$ and the $\mathrm{pH}$ values were measured using a Minitrode $\mathrm{pH}$ electrode (Hamilton). The measurements were performed at $20^{\circ} \mathrm{C}$ with a path length of $10 \mathrm{~mm}$ and recorded between $250-400 \mathrm{~nm}$. a.) Near-UV CD spectra for the $\mathrm{pH}$ titration experiment on human TK Q428A. Inset: $\mathrm{pH}$ dependence of AP formation at 325-335 $\mathrm{nm}$ (average value). The $\mathrm{pKa}$ was determined using equation 5a. b.) Human TK S427A. c.) Human TK H77A. d.) Human TK G123A. 


\section{Discussion}

\subsection{Function of the low barrier hydrogen bond (LBHB) in $h \mathrm{TK}$}

\subsubsection{Crystallographic studies on the LBHB}

The catalytic function of LBHBs during enzymatic reaction was discussed extensively during the past twenty years. The concept that LBHBs play a pivotal role in enzyme catalysis, especially in the reaction of serine protease, has been accepted by some scientists but rejected by others (Frey et al., 1994; Cassidy et al., 1997; Fuhrmann et al., 2006; Tamada et al., 2009). In this study, we have successfully determined the crystal structure of human transketolase at ultra-high resolution. By careful analysing the structure of $h \mathrm{TK}$ in complex with the physiological substrate X5P, a LBHB was observed between the conserved Glu366 and a neighbouring Glu160 (Fig 13). The distance between the H-bond donor and acceptor is around $2.56 \AA$ and the electron density for the shared hydrogen atom could be observed as well at nearly middle position between the H-bond. LBHBs have been observed by protein Xcrystallography in other enzymes such as serine protease at $0.78 \AA$ (Kuhn et al., 1998), $\beta$ lactamase at (Nichols et al., 2015) and phosphate binding proteins at $0.98 \AA$ and $0.88 \AA$ (Elias et al., 2012). Photoactive yellow protein (PYP) was also proposed to contain a LBHB, which was revealed by high-resolution neutron crystallography (Yamaguchi et al., 2009). Our results represent the observation of a LBHB in thiamine-dependent enzymes for the first time.

As shown in Fig 14, the two active sites of $h \mathrm{TK}$ are connected by a "proton wire" which consists of several acidic amino acid residues and water molecules. This structural feature has been observed in several ThDP-dependent enzymes. An identical "proton tunnel" synchronizing the active sites in the E1 component of pyruvate dehydrogenase complex has been observed and proposed to conduct protons from one ThDP to the other for the "half-ofthe-sites reactivity" (Frank et al., 2004; Jordan, 2004). Our finding of a LBHB in this proton wire in transketolase would bring a new concept in understanding the proton transfer mechanism in this "tunnel". The activation of thiamine in ThDP enzymes starts from the donation of a proton from the conserved glutamate to the N1' of the aminopyrimidine, thus Glu366 becomes deprotonated and has the potential to take its proton back. To compensate the loss of proton on Glu366, the neighbouring Glu160 shares half of its proton and forms the short-distanced LBHB with Glu366. Therefore, the catalytic role of the LBHB between Glu160 and 366 would be to stabilize the deprotonated Glu366 and prevent it to take back the proton on N1' of aminopyrimidine, thus ensuring the catalytic efficiency. In this manner, E160 could be referred as a "sensor" which can feel the deprotonated state of Glu366 and reacts to 
it by forming the LBHB. Glu165 interacts with the "sensor" Glu160 via a water molecule and might also play a role in stabilizing the conformation of Glu160.

Sequence alignment (Fig 54) reveals that the three glutamates located in the proton wire "tunnel" are conserved among transketolases from different species, which indicates the unreplaceable role for these conserved glutamates and possible existence of LBHBs at the same position. An analysis of the hydrogen bond length at the same LBHB position in all transketolase structures with a resolution higher than $2.0 \AA$ reveal a commom short hydrogen bond feature (Table 10). A crystal structure of EcTK (EcTK D469N at $1.06 \AA$ ) has revealed the formation of a LBHB between Glu160 and Glu411 (Glu366 in human TK), same position as in the structure of human TK (Fig 59). These results together suggest a high possibility of LBHBs existing in all transketolases and playing the same catalytic role as in the case of human TK. To confirm the LBHBs in transketolases in other species, improving the resolution of crystal structures is therefore needed.

\begin{tabular}{|lll|}
\hline Homo sapiens & 154 GDGELSEGSVWEAMAFASIYKLD - 358 RFIECYIAEQNMVSIAVGCATRN \\
E. coli & 154 GDGCMMEGISHEVCSLAGTLKLG - 403 NYIHYGVREFGMTAIANGISLHG \\
S. cerevisiae & 156 GDGCLQEGISSEASSLAGHLKLG - 410 RYIRYGIREHAMGAIMNGISAFG \\
M. tuberculosis & 176 SDGDIEEGVTSEASSLAAVQQLG - 430 RTLHFGVREHAMGAILSGIVLHG \\
L. salivarius & 172 GDGDLMEGVASEAASLAGHLKLG - 426 RNIWFGVREFGMACAMNGIMLHG \\
Z. mays & 164 GDGCQMEGIANEACSLAGHWGLG - 443 RNVRFGVREGMGAICNGIALHS \\
P. aeruginosa & 159 GDGCMMEGISHEVASLAGTLRLN - 409 NYVFYGVREFGMSAIMNGVALHG \\
\hline
\end{tabular}

Fig 54: Sequence alignment of transketolases from different species. The three glutamate residues (E160, E165 and E366 in human TK) in the proton wire "tunnel" between the two active sites are conserved in transketolases sequences from different origins (Asztalos et al., 2007; Fiedler et al., 2002; Fullam et al., 2012; Gerhardt et al., 2003; Lukacik et al., 2015; Mitschke et al., 2010; Veitch et al., 2004). The multiple sequence alignment was performed by using the Clustal Omega online server (Larkin et al., 2007). Yellow: fully conserved residues; Blue: residues with similar properties; Green: residues with weakly similar properties. The three conserved glutamates are shown in bold.

After observation of the LBHB in $h \mathrm{TK}$, an active site mutant E160Q was generated and the crystal structure at $1.06 \AA$ clearly indicates an ordinary H-bond at the same position where the LBHB exists in the wild type (Fig 15). In addition, IP form was observed for the six-membered aminopyrimidine ring for both wild type and E160Q variant, which is consistent with previously reported results (Lüdtke et al., 2013). Closer analysis of the obtained donor substrate F6P-ThDP intermediate also revealed the distorted intermediate and elongated scissile bond. Furthermore, the scissile $\mathrm{C} 2 \mathrm{x}-\mathrm{C} 3 \mathrm{x}$ bond of the F6P-ThDP intermediate in the crystal structure of $h \mathrm{TK}$ E160Q variant is found to be perpendicular relative to the fivemembered thiazolium ring (Fig 17). This structural orientation would help the direct 
conjugation of the electron pair generated on bond cleavage with the aromatic thiozolium ring's $\pi$ electrons, which is a well-accepted mechanism named "maximum overlap" mechanism (Friedemann and Breitkopf, 1996). Analysis of the bond lengths of the F6P-ThDP intermediate reveals that the $\mathrm{C} 2 \mathrm{x}-\mathrm{C} 3 \mathrm{x}$ bond, which will be cleaved in the following steps, is elongated to around $1.60 \AA$. This is atypical for the C-C single bonds in sugars (1.52-1.53 $)$ while the bond lengths of other substrate $\mathrm{C}-\mathrm{C}$ single bonds as well as the $\mathrm{C} 2-\mathrm{C} 2 \mathrm{x}$ bond which links the substrate with thiazolium ring are in the range of normal bond lengths (Allen et al., 1987). The bond length elongation would decrease the dissociation energy (0.10-0.15 corresponds to $\sim 30 \mathrm{kcal} / \mathrm{mol}$ ) (Zavitsas, 2003), thus lowers the needed energy for the subsequent steps and leads the enzymatic catalysis more efficient. Although the structures of E160Q with bound X5P and S7P were not determined, based on the structures of wild type the same behaviour could be proposed.

The F6P-ThDP intermediate forms multiple hydrogen bonding interactions with the active site residues of $h \mathrm{TK}$ (Fig 18). These interactions are identical to the similar F6P-ThDP intermediate obtained in the crystal structure of E.coli TK (Asztalos et al., 2007). The only difference is that Gln428, which adopts two alternative conformations, was observed in $h \mathrm{TK}$ while His 473 is found in E.coli TK at the same position. The observation of two alternative conformations of Gln428 is reminiscent of the ring-flipping behaviour of His473 in E.coli TK (Neumann and Tittmann, 2014), which reflects that the dynamics of the active site residue of this position in transketolase from different species seem to share a common function in the transketolase family. Interestingly, the F6P-ThDP intermediate could form an intra-molecular hydrogen bonding interaction between the $\mathrm{O} 1 \alpha$ of sugar and N4' of aminipyrimidine, which might account for the stabilization of the $\mathrm{C} 2-\mathrm{C} 2 \mathrm{x}$ angular distortion. The crystal structure of $h$ TK-E160Q variant at $1.04 \AA$ revealed similar active site architecture to that of the structure in complex with donor F6P (Fig 19), indicating that substrate binding does not affect the hydrogen bonding interaction at the LBHB position.

The LBHB in $h$ TK wild type is formed between two glutamates, Glu160 and Glu366. The crystal structure of E160Q variant revealed the replacement of the LBHB by an ordinary hydrogen bond. In the next step, the E366Q variant was generated and its crystal structure with donor substrate X5P was determined at $1.5 \AA$ A. Interestingly, the donor X5P does not form covalent intermediate with cofactor but exists as the acyclic keto form at the docking site (Fig 20). This observation is consistent with the well-accepted mechanism that Glu366 is crucial for the cofactor activation (Kluger and Tittmann, 2008). The inter-atomic distance between the $\mathrm{C} 2$ reactive center of ThDP and the carbonyl $\mathrm{C} 2$ atom of $\mathrm{X} 5 \mathrm{P}$ is around $3.63 \AA$, while the optimal inter-atomic distance between the two reactants for a typical nucleophilic attack is 1.5 
to $3 \AA$ (Burgi et al., 1973; Burgi et al., 1974). In addition, the optimal value for the relative orientation of the two reactants is around $107^{\circ}$ but a value of $58^{\circ}$ is observed for the angle of $\mathrm{C} 2$ (ThDP), C2 (X5P) and O2 (X5P). Based on these two deviations from optimal values, it is reasonable to assume that a spatial rearrangement of either the donor substrate and/or the cofactor is necessary in the subsequent steps to form the covalent donor-ThDP intermediate.

The presence of a glutamate forming hydrogen bonding interaction with the N1' of the aminopyrimidine ring is found to be a conserved element in several ThDP dependent enzyme of which crystal structures are already determined (Kluger and Tittmann, 2008; Schellenberger, 1998). Site-directed mutagenesis studies have revealed the catalytic importance of this glutamate in promoting $\mathrm{C} 2-\mathrm{H}$ ionization and the subsequent activation of the cofactor (Shaanan and Chipman, 2009). The fundamental role has been further characterised by UV-vis and CD spectroscopic studies involving the tautomerization of the 4'aminopyrimidine and 1', 4'-iminopyrimidine (Nemeria et al., 2004, 2007). However, the crystal structure of glyoxylate carboligase had revealed a valine residue at the position of the conserved glutamate and re-introducing a carboxylate by mutagenesis would outweigh the advantage of accelerating the $\mathrm{C} 2-\mathrm{H}$ ionization (Kaplun et al., 2008; Shaanan and Chipman, 2009).

\subsubsection{Functional studies on the LBHB and related variants}

In the previous chapter, the steady-state kinetics of $h \mathrm{TK}$ wild type and LBHB-related active site variants were measured based on an assay using two auxiliary enzymes. Compared with wild type, the E160Q and E160A variants showed an overall activity of $20 \%$ and $8 \%$, respectively. But the substrate affinity of these two mutants are very similar to that of the wild type, suggesting that Glu160 in $h \mathrm{TK}$ is not responsible for substrate binding. Identical singlemutation variants for yeast TK at the same position have already been studied. Results from Meshalkina and colleagues (Meshalkina et al., 1997) also revealed similar substrate binding affinity of this conserved glutamate (Glu162 in yeast TK), but the impact on the turn over number $k_{\text {cat }}$ is less pronounced (70 \% for E162Q, $46 \%$ for E162A) compared with human TK. Another study on the E.coli PDH complex E1 component showed similar turn over number ( $10 \%$ ) but increased $\mathrm{Km}$ value ( 3 fold) for its substrate pyruvate (Nemeria et al., 2010). The $h$ TK-E165Q showed only $4 \%$ overall activity compared with the wild type enzyme, which suggests a pivotal role for this residue. Studies of the E.coli PDH complex E1 component at the same position revealed similar impact and showed impaired activity of the binding of ThDP at the second active site, suggesting that this residue is responsible for the active sites communication (Nemeria et al., 2010). The same was studied on the E.coli TK which revealed that Glu165 is pivotal for dimerization to form the active dimer (unpublished data). For the 
conserved glutamate 366, an enzyme variant E366Q was generated and steady-state kinetic analysis showed only $0.5 \%$ residue activity compared with the wild type. The functional role of this residue for other ThDP-dependent enzymes has been extensively studied, for example Zymomonas mobilis PDC E50Q (<0.5\%) (Candy et al., 1996), IPDC E52D (0.1\%) (Schütz et al., 2005b), DXS E370Q ( 0) (Xiang et al., 2007), E.coli PDH E1 E571A (1.7 \%), E571D (0.61\%), E571Q (1.3\%) (Nemeria et al., 2010). All these results support the fundamental role of the conserved glutamate for ThDP activation and partially rule out the proposal that a histidine and water molecule dyad are responsible for the ThDP C2 deprotonation by using Quantum Mechanical/Molecular Mechanical Method (Nauton et al., 2016; White et al., 2016). Interestingly, a lag phase could be observed for $h$ TK-E160Q variant when low donor substrate X5P concentration $(10 \mu \mathrm{M})$ was used (Fig 23). Such lag phase has been observed in other enzymes such as Thermoplasma acidophilum transaldolase. In this enzyme, the lag phase most likely reflects the conformational dynamics of the protein that samples between an "open" and "closed" state (Sautner et al., 2015). For the origin of the lag phase in $h \mathrm{TK}$, it might reflect the non-equivalence of the two active sites, but this needs to be further studied.

Taking the concept of low barrier hydrogen bonds (LBHBs) into consideration, the sitedirected mutation of Glu160 into Gln160 simply exchanges the LBHB with an ordinary hydrogen bond which is revealed by the X-ray crystallography structures. Steady-state kinetic analysis reveals a 5-fold effect of the LBHB over the ordinary hydrogen bond for the substrate conversion in enzyme catalysis. Similar results were reported by Elias. $\mathrm{M}$ and colleagues that single mutation of Asp62 which forms a LBHB with substrate into Asn decreases the selectivity between phosphate and arsenate 4-10 fold (Elias et al., 2012). Do song jang and colleagues reported the absence of a LBHB in ketosteroid isomerase reduced the catalytic activity by only a factor of 1.7-2.0 (Jang et al., 2015). The general role of LBHBs in enzymatic catalysis therefore varies slightly among different enzyme families.

In the following step, the donor half reaction of $h \mathrm{TK}$ was studied by a single-mixing stoppedflow experiment. The depletion of AP and the formation of IP were measured at 325 and 295 $\mathrm{nm}$, respectively. Interestingly, the reaction of E160Q and E160A showed a two-phase process while only one phase was observed for the wild type (Fig 24). The possible explanation for the first phase would be that before the covalent addition between F6P and ThDP takes place, a pre-equilibrium process has happened. Binding of F6P induces the chemical rearrangement of $\mathrm{AP}$ ring from $\mathrm{APH}^{+}$to $\mathrm{AP}$ and IP (also shows biphasic property, data not shown), a very fast procedure which finishes in $30 \mathrm{~ms}$. The uncommon existence of the pre-equilibrium phase on E160Q/A variants suggests a $\mathrm{pKa}$ change relative to the wild type, which is further supported by the Circular Dichroism (CD) spectroscopy based $\mathrm{pH}$ titration results. 
In general, the donor half reaction of transketolase could be interpreted as a reversible twostep mechanism with transient formation of a Michaelis complex as a preceding rapid equilibrium (Fig 55). This two-step reaction is better observed on the E160Q and E160A variant measured at $325 \mathrm{~nm}$ than the wild type. The first fast process (around $30 \mathrm{~ms}$ ) represents the rapid formation of the E-ThDP*F6P Michaelis complex which induces a pre-equilibrium of the tautomeric and protonic states of the six-membered aminopyridine ring. The second phase for the formation of E-ThDP-F6P covalent adduct is accompanied by the depletion of the AP form and leads to the accumulation of the IP form. High resolution X-ray structures of X5P-ThDP, F6P-ThDP, S7P-ThDP covalent adducts confirm the concomitant accumulation of the IP form for the six-membered ring (Lüdtke et al., 2013).

$$
\begin{gathered}
\text { E-ThDP + F6P } \stackrel{K_{\mathrm{s}}^{\text {app }}}{\rightleftharpoons} \text { E-ThDP*F6P (Michaelis complex) } \\
\text { E-ThDP*F6P } \underset{k_{-2}}{\stackrel{k_{\text {obs }}}{\rightleftharpoons}} \text { E-ThDP-F6P (covalent adduct) }
\end{gathered}
$$

Fig 55: Minimal reaction sequence for the pre-steady-state reaction of transketolase (donor half reaction). Donor substrate F6P and the holo-transketolase first undergo a transient formation of a Michaelis complex which is further converted into the donor substrate-ThDP covalent adduct with a reaction constant of $k_{\mathrm{obs}} . K_{\mathrm{S}}^{\text {app }}=$ the dissociation constant of the pre-equilibrium. $k_{-2}=$ the rate constant of the carbonyl elimination.

Taking the steady-state kinetic data into consideration, E160Q and E160A variants have an overall activity around $20 \%$ and $7 \%$ of the wild type, while the pre-steady-state analysis reveal $65 \%$ and $90 \%$ activity of wild type. These data suggest that the rate-limiting step for human TK doesn't lie on the donor half reaction but rather in the subsequent acceptor half reaction. For the reaction of E.coli TK, detailed kinetic analysis of microscopic reaction steps revealed that the rate-limiting step is the release of product S7P which is the last step of the whole reaction cycle (Lüdtke, 2012). Therefore, we may also propose the same rate-limiting step but further kinetic analysis for the acceptor half reaction is needed.

The six-membered aminopyrimidine ring of ThDP undergoes three tautomeric and protonation states during catalysis. In a previous study, the $\mathrm{pKa}$ of the $([\mathrm{AP}]+[\mathrm{IP}]) /\left[\mathrm{APH}^{+}\right]$equilibrium was determined to $5.82 \pm 0.02$ according to a CD-based $\mathrm{pH}$ titration experiment ((Lüdtke et al., 2013). In order to explore the impact of the $\mathrm{LBHB}$ on the ([AP] + [IP])/[APH $\left.{ }^{+}\right]$equilibrium, the same $\mathrm{pH}$ titration experiments were performed on LBHB-related active site variants. The CD spectra of $h$ TK-E160Q revealed a dramatically different feature compared with the wild type. For wild type, a negative band appears at $290 \mathrm{~nm}$ and the amplitude decreases with $\mathrm{pH}$ values (Fig 28). This band is assigned as the AP form with the protonated glutamate (Fig 27). Taking the LBHB between Glu366 and Glu160 into consideration, when Glu366 donates a 
proton to the N1' of ThDP, Glu160 would share its proton with Glu366 by forming the LBHB to stabilize the deprotonation state of Glu366. The crystal was obtained at the $\mathrm{pH}$ where the ThDP should exist in the AP form with deprotonated glutamate, which is consistent with the CD spectroscopic observation. For the $h$ TK-E160Q variant, the band at $290 \mathrm{~nm}$ could be observed even at low $\mathrm{pH}(<5.0)$ and the amplitude decreases with $\mathrm{pH}$ values. This is also consistent with the crystal structure of E160Q with bound F6P in which an ordinary hydrogen bond has replaced the LBHB. Glu366 can't fully maintain the deprotonated state without the assistance of the LBHB after donating its proton to thiamine especially at low $\mathrm{pH}$, therefore only AP form with protonated glutamate was observed at $\mathrm{pH}$ 5.0. When $\mathrm{pH}$ value goes up, glutamte366 would shift from the protonated to the deprotonated form with AP for the aminopyrimidine ring. The $\mathrm{pKa}$ for the ([AP] $+[\mathrm{IP}]) /\left[\mathrm{APH}^{+}\right]$equilibrium of $h \mathrm{TK}-\mathrm{E} 160 \mathrm{Q}$ was determined to $7.23 \pm 0.06$.

Literature reports that a LBHB could store $10-20 \mathrm{kcal} / \mathrm{mol}$ energy while an ordinary hydrogen bond could only store $5 \mathrm{kcal} / \mathrm{mol}$ energy (Cleland and Kreevoy, 1994; Warshel et al., 1995). So the energy difference between the two is around $5-15 \mathrm{kcal} / \mathrm{mol}$. The relation between the Gibbs free energy and $\mathrm{pKa}$ constant is shown in equation 6 from which the energy difference of wild type and E160Q is calculated to $2.01 \pm 0.01 \mathrm{kcal} / \mathrm{mol}$. This value reflects the general variation of the ionization properties of the active-site residues between wild type and E160Q and couldn't be simply attributed to a LBHB or an ordinary hydrogen bond. But considering the fact that the micro-environment of the active site is determined by all the amino acid residues though a hydrogen bonding network and one-single mutation sometimes has a huge impact, we could assign the overall energy change to local hydrogen bonding interactions and conclude an experimentally observed energy difference between a LBHB and an ordinary hydrogen bond of $2.01 \pm 0.01 \mathrm{kcal} / \mathrm{mol}$. This value has brought a different opinion to the canonical energy profile for LBHBs which is obtained from computational calculation (Warshel et al., 1995).

$$
\Delta \mathrm{G}=-2.303 \mathrm{RT} \mathrm{p} K a
$$

Equation 6: Gibbs free energy $\Delta G$. $R=$ gas constant; $T=$ absolute temperature.

As shown in Fig 28, the full amplitude of AP form for $h$ TK-E160Q is only half of the wild type, supporting the "half-of-the-sites" reactivity mechanism for ThDP enzymes. The active sites communication might be interrupted by mutation, which can be further confirmed by the temperature jump experiment (Fig 30, 60) where the E160Q variant shows no chemical 
equilibrium shift for $([\mathrm{AP}]+[\mathrm{IP}]) /\left[\mathrm{APH}^{+}\right]$. The $\mathrm{pH}$ titration $\mathrm{CD}$ spectra of $h \mathrm{TK}-\mathrm{E} 366 \mathrm{Q}$ exhibits dramatic difference compared with other variants. At the whole $\mathrm{pH}$ titration range, the AP form with deprotonated glutamate at $325 \mathrm{~nm}$ could not be observed, while AP form with protonated glutamate at $290 \mathrm{~nm}$ stays the same amplitude. The lack of the AP band with deprotonated glutamate thus could not activate the cofactor, leading this variant almost dead. The crystal structure also supports this proposal with the capture of X5P at the docking site instead of the covalent X5P-ThDP intermediate. In addition, the amide group of Gln366 could be clearly assigned to the position that hydrogen bonds with $\mathrm{N} 1$ ' of thiamine. If the $\mathrm{APH}^{+}$form were accumulated on the enzyme, the hydrogen atom on the N1' will clash with the two hydrogens on the amide of Gln366, which again rules out the formation of $\mathrm{APH}^{+}$form. Observation of the AP band with protonated glutamate (glutamine) by CD spectroscopy was earlier revealed on EcTK E411A variant (Asztalos, 2008).

In previous chapter, a temperature jump ( $\mathrm{T}$-jump) technique was employed to analyse the tautomeric and protonation states of ThDP with an ultra-fast temperature rise of $10{ }^{\circ} \mathrm{C}$. The AP and IP tautomer both decrease with the temperature jump and exhibit similar rate constants (Fig 30, $11559 \pm 408 \mathrm{~s}^{-}$and $13950 \pm 3417 \mathrm{~s}^{-}$for AP and IP, respectively). Since both the AP and IP tautomer decrease when temperature rises, it is reasonable to assume that the chemical equilibrium shifts towards the $\mathrm{APH}^{+}$form even though there is no direct method to detect it so far. The increase of temperature favours the formation of the $\mathrm{APH}^{+}$form which indicates that it is an endothermic reaction to form $\mathrm{APH}^{+}$while formation of AP and IP are exothermic.

In the last step, intermediate distribution of $h \mathrm{TK}$-wt and LBHB-related active site variants were analysed according to a NMR based acid-quench method. This technique enables quantitative analysis of covalent intermediates during ThDP enzyme catalysis and can also assign functions for individual residues if related active site mutants are studied (Tittmann et al., 2003). The spectra of wild type reveals the accumulation of $72 \%$ of F6P-ThDP covalent intermediate, $12 \%$ of the post-cleavage DHEThDP intermediate and $16 \%$ of the Michaelis complex (indicated by free ThDP). This NMR result is consistent with the single-mixing stopped-flow kinetics of the donor half reaction using F6P mentioned in the previous chapter. At equilibrium, the $k_{\mathrm{obs}}{ }^{\max }$ for formation of the covalent F6P-ThDP adduct is around $9.06 \pm$ $0.72 \mathrm{~s}^{-}$, which is approx. 3 fold of the velocity for the reverse reaction $k_{-2}\left(2.84 \pm 0.53 \mathrm{~s}^{-}\right)$. The 1:3 distribution between F6P-ThDP and Michaelis complex matches the kinetics very well. For E160Q and E160A variants, only the F6P-ThDP covalent adduct could be observed, which is also consistent with donor half reaction kinetics that the reverse reactions are very slow for these two variants $\left(0.47 \pm 0.24 \mathrm{~s}^{-}\right.$for E160Q and $0.62 \pm 0.49 \mathrm{~s}^{-}$for E160A). In addition, a small fraction $(12 \%)$ of DHEThDP intermediate is observed on $h$ TK-wt. The DHEThDP 
intermediate is the nucleophile which will attack the aldehyde group of aldose phosphate sugars in the acceptor half reaction. If some fraction of it already appears in the donor half reaction, it will readily help the catalytic efficiency a lot when the acceptor substrates bind to the active site. This is revealed by the steady-state kinetics as well as the pre-steady-state kinetics. For the donor half reaction, E160Q and E160A show approx. $65 \%$ and $90 \%$ maximal velocity relative to wild type respectively. However, steady-state kinetic analysis reveals that E160Q exhibits only $20 \%$ activity while E160A has an even lower catalytic efficiency of 10 $\%$ compared with wild type. The drop of catalytic power must come from the cleavage of donor-ThDP intermediate and the acceptor half reaction. Regarding of those perspectives, the accumulation of DHEThDP intermediate in wild type would make contribution to the overall catalytic efficiency therefore has its meaning of existence.

\subsection{Capture of the central dihydroxyethyl thiamine diphosphate (DHEThDP) intermediate from non-phosphorylated substrates}

\subsubsection{Accumulation of the DHEThDP intermediate in the crystal structure of human TK with xylulose and fructose}

The existence of DHEThDP intermediate has been textbook knowledge for years but remained elusive until Fiedler and colleagues (Fiedler et al., 2002) captured the three dimensional structure of this intermediate at the active site of yeast TK by cryocrystallography. The $1.9 \AA$ crystal structure suggested that the $\mathrm{C} 2-\alpha$ carbanion/enamine intermediate existed as planar and E-configuration form. Later on in 2010 Suzuki and colleagues (Suzuki et al., 2010) observed the DHEThDP intermediate in phosphoketolase with a $\mathrm{sp}^{3}$ hybridized $\mathrm{C} 2$ atom which indicates non-planarity of the DHE moiety. Our previous colleagues confirmed the non-planarity of this intermediate by resolving sub-angstrom resolution crystal structures of $E c$ TK socked with artificial substrate hydroxypyruvate (HPA) (Lüdtke, 2012). Since the DHEThDP intermediate is crucial in the thiamine catalysis, in this thesis we have identified a new route to capture this pivotal intermediate from non-phosphorylated sugars xylulose and fructose.

In addition, previous studies have shown that HPA is a suitable ketol donor substrate used to trap this particular intermediate DHEThDP. HPA first binds to the $\mathrm{C} 2$ position of ThDP and forms a short-lived intermediate hydroxylactyl-ThDP which undergoes decarboxylation to form DHEThDP by concomitant release of carbon dioxide as a by-product. Due to the virtue of the irreversibility of decarboxylation reaction, HPA is generally used for the asymmetric carbon-carbon formation in organic synthesis (Hailes et al., 2013). HPA is commercially 
available and relatively cheap, but it would not do any harm if a much cheaper ketol donor substituent could be found.

In this study, the accumulation of the post-cleavage DHEThDP intermediate using xylulose and fructose could be confirmed first by an acid quench/1H NMR measurement (Fig 33). Comparison of non-phosphorylated sugars with HPA, some advantages are obviously seen. First, the carbanion/enamine intermediate obtained from fructose/xylulose seems to be more stable than that from HPA which only has a half-time of $120 \mathrm{~s}^{-}$(Lüdtke, 2012). Secondly, using of HPA would generate some other intermediates such as erythrulose-ThDP due to several offpathway reactions (Asztalos, 2008), while the non-phosphorylated sugars only induce the formation of the desired intermediates. Thirdly, for biosynthetic purposes the availability of the six-carbon sugar fructose proves itself a much cheaper donor substrate for TKs relative to that of HPA. Although this combined acid quench/1H NMR method is very powerful in the analysis of different intermediates distribution of ThDP-dependent enzymes, it comes with limitation. Quench solution with strong acidity ( $\mathrm{pH} 0.75$ ) will render all compounds into conjugated acid which does not permit the resolution of their protonation states. Therefore, the carbanion, enamine or protonated form of DHEThDP can not be discriminated by this approach (Tittmann et al., 2003).

In the next step, the crystal structures of $h \mathrm{TK}$ with non-phosphorylated sugars xylulose and fructose are determined to medium resolution $(\sim 1.5 \AA)$. For both structures, the electron density at the $\mathrm{C} 2$ of the cofactor ThDP clearly indicates the formation of the DHEThDP intermediate with high occupancy (Fig 35, 62, 70-80 \% and 60-70 \% for xylulose and fructose, respectively). But there is one thing to consider at this point, acetyl-ThDP (AcThDP), the dehydration form of the DHEThDP intermediate in the pathway of phosphoketolase reaction, has a similar chemical structure to the enamine/carbanion intermediate (Suzuki et al., 2010). Since it could not be detected by NMR intermediate measurement, the existence of the AcThDP form therefore could not ambiguously excluded by the X-ray crystallography data. Closer inspection of the structures reveals that the $\mathrm{C} 2-\mathrm{C} 2 \alpha$ bond connecting cofactor and the DHE moiety exhibits an out-of-plane distortion (Fig 35). This partially suggests a tetrahedral arrangement of the bonding at $\mathrm{C} 2$, leading to the loss of aromaticity of the thiazolium ring. Closer inspection reveals that $\mathrm{C} 2$ atom exhibits deviation from the thiazolium plane, which further supports the nonaromatic feature of the thiazolium ring. This observation has revised the knowledge that planar enamine state of DHEThDP intermediate exists in ScTK at a resolution of $1.9 \AA$ (Fiedler et al., 2002). For the six-membered aminopyrimidine ring, deviations could also be observed for some atoms (Fig 35, N3', C2'-M, C7'). Previous studies on pyruvate oxidase (POX) (Meyer et al., 2012) revealed that atom N4' of the 4'-amino group 
deviates by approx. $10^{\circ}$ from the aminopyrimidine plane, an interesting feature that is different from the enamine intermediate in $h \mathrm{TK}$. This adopted high energy conformation is thought to facilitate the interconversion between various tautomeric states of the aminopyrimidine. Therefore, we could speculate the deviation of the aminopyrimidine ring in the $h \mathrm{TK}$ DHEThDP intermediate also play similar role during catalysis but this hypothesis remains to be tested.

As mentioned above, the bond lengths suggest the double bond character of the $\mathrm{C} 2-\mathrm{C} 2 \alpha$ bond thereof sp2 hybridization of $\mathrm{C} 2 \alpha$ atom, while the out-of-plane features partially indicate a tetrahedral arrangement of the bonding with sp3 hybridization at $\mathrm{C} 2 \alpha$ atom. To distinguish between these two states, the geometry of N3 is employed as reference for the electron distribution. Interatomic distance of $1.44 \pm 0.02 \AA$ between $\mathrm{C} 2$ and N 3 indicates single bond character (1.31 A for the $\mathrm{C} 3-\mathrm{N} 3$ double bond observed in small molecule structure of thiamine models). Based on this, a more enamine-like structure should fit better to the electronic state of the DHEThDP intermediate.

In addition, the two leaving groups which are D-glyceraldehyde from xylulose and Derythrose from fructose are also captured within the crystal structures (Fig 37, 38). The aldehyde trioses or tetroses would form the hydrate form or dimerize in aqueous solutions, as studies have shown that D-glyceraldehyde and D-erythrose mainly consist of mixtures of the dimeric forms in the syrupy state (Serianni et al., 1979). But we only capture the monomeric aldehyde form of these sugars in the crystal structure, suggesting that the active site of enzyme could sequester water molecules outside the protein and prevent hydration reaction to take place. In addition, the microenvironment of the active site does not provide enough space to accommodate two molecules of the released aldehyde sugars to form the dimeric form.

In order to analyse the structural changes of the cofactor ThDP upon the formation of the covalent intermediate DHEThDP intermediate by using xylulose and fructose, structures of $h$ TK soaked with both sugars are superimposed with holo- $h$ TK (1.75 A, PDB: 3MOS). Differences for the thiazolium ring (TH) as well as for the aminopyrimidine ring (AP) are observable whereas the diphosphate anchor (PP) is perfectly superimposable between both molecules. Slightly pronounced deviation for the planarity of $\mathrm{C} 2 \alpha$ atom for the structure soaked with fructose suggests a higher fraction of $\mathrm{C} 2 \alpha$ carbanion for this structure. 


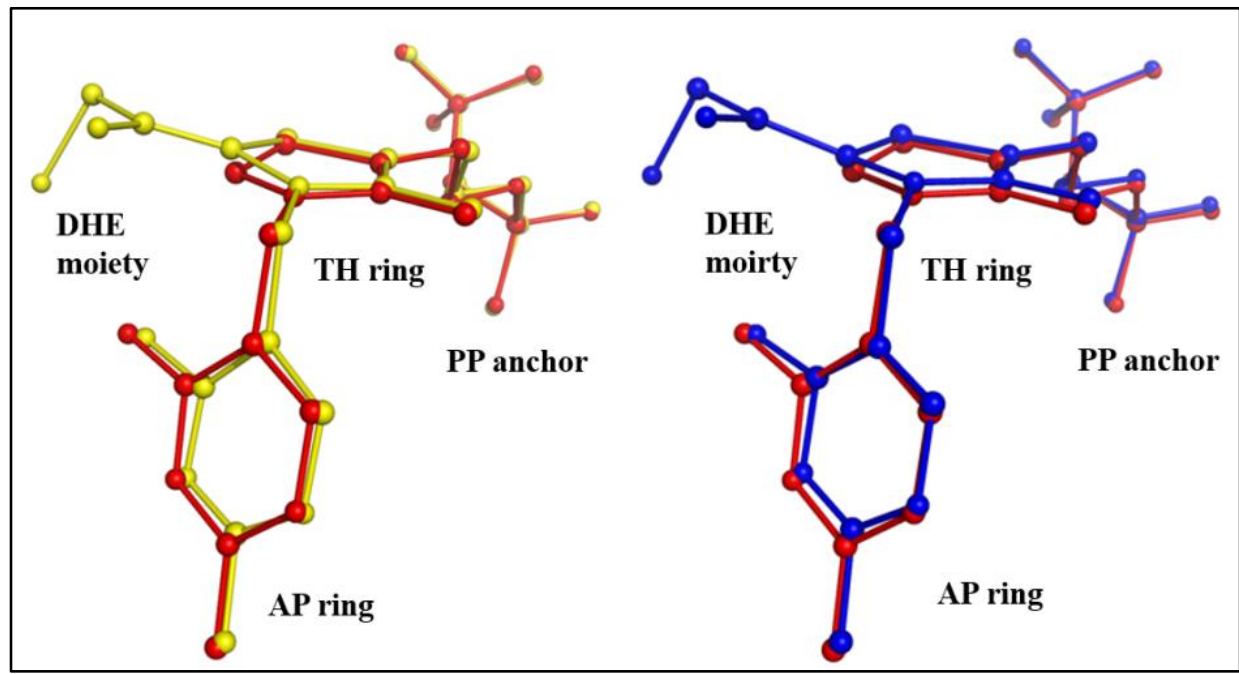

Fig 56: Superposition of the ThDP molecule in holo- $h \mathrm{TK}$ (red) and covalent reaction intermediates DHEThDP in $h$ TK soaked with xylulose (left panel, yellow) and fructose (right panel, blue). In order to visualize structural changes upon the formation of the DHEThDP intermediates by using the 5-carbon sugar xylulose and 6-carbon sugar fructose, both structures are superimposed with holo $h \mathrm{TK}$. All structures are represented in ball-stick model, aminopyrimidine (AP ring) and thiazolium (TH ring) as well as the diphosphate moiety (PP anchor) are labelled.

Furthermore, the overall structure of the polypeptide chain in these two structures are very similar to the structure of holo- $h$ TK in the resting state (RMSD of $0.35 \AA$ and $0.41 \AA$ for the structures of xylulose and fructose respectively for the monomer), suggesting that the active site is poised to accept donor substrate and conformational changes are not necessary during the enzyme reaction. In addition, both structures were found to be present in the monoclinic space group P21 with two monomers per asymmetric unit. The DHEThDP intermediate are present in both active sites with similar occupancy, ruling out the previous proposals of halfof-the-sites reactivity in ThDP enyzmes (Frank et al., 2007a), at least, in human transketolase.

\subsubsection{DHEThDP intermediate exists as an enolate form}

Previous studies of the DHEThDP intermediate on EcTK obtained at super-high resolution with HPA have revealed that an enolate form exists as the predominant state of the intermediate (Lüdtke, 2012). By comparing the bond lengths of dihydroxyethyl moiety of DHEThDP intermediates obtained from non-phosphorylated sugars with this structure we concluded that the newly-obtained structures could also be interpreted as the enolate form. The formation of enolate could potentially prevent the protonation of the highly reactive $\mathrm{C} 2 \alpha$ carbanion. Other chemical states such as the ketone form suggested by analogous hydroxyethyl-ThDP (HEThDP) intermediate in LpPOX (Meyer, 2012) could be ruled out for 
two reasons: a.) as an electrophilic substrate center the ketone form will not be able to attack the carbonyl group of the acceptor, b.) the double bond character for the $\mathrm{C} 2-\mathrm{C} 2 \alpha(1.35 \pm 0.02$ $\AA$ ) is incompatible with the structure of the ketone.

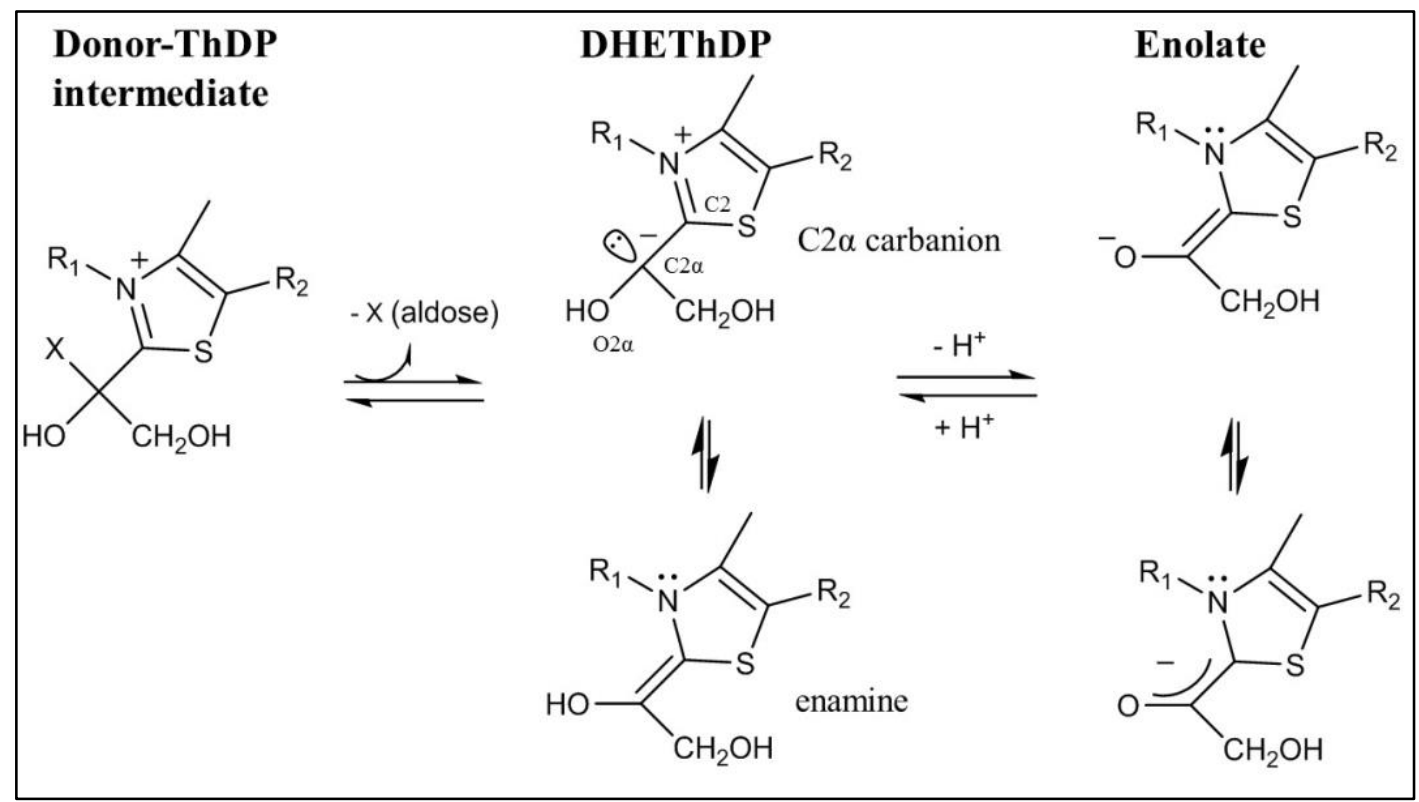

Fig 57: Mechanism for the formation of enolate form of DHEThDP. After the cleavage of the donorThDP intermediate, the $\mathrm{O} 2 \alpha$ of the enamine/carbanion could be deprotonated by a general base to form an enolate form. Selected chemical structures and atoms are labelled $\left(\mathrm{R}_{1}=\right.$ aminopyrimidine, $\mathrm{R}_{2}=$ ethyldiphosphate).

The DHEThDP enolate form could be generated by a mechanism which is shown in Fig 57. After the cleavage of the donor-ThDP intermediate, the enamine/C $2 \alpha$-carbanion was formed and the subsequent deprotonation of $\mathrm{O} 2 \alpha$ could be accomplished by a base from the surrounding environment. Gln428 resides at the position where a hydrogen bonding interaction is possible, but glutamine residues cannot act as acid/base catalysts. Closer inspection reveals that an intramolecular proton transfer from $\mathrm{O} 2 \alpha$ to the $\mathrm{N}^{\prime}$ ' position of the aminopyrimidine ring most likely accounts for the formation of the enolate. This interaction should be feasible based on the inter-atomic distances ( $3.26 \AA$ in both structures from fructose and xylulose), and identical structural features (3.06 ̊ for $h \mathrm{TK}, 3.11 \AA$ for $E c \mathrm{TK}$ and $2.98 \AA$ for $S c \mathrm{TK}$ ) had been observed for the DHEThDP intermediate obtained from HPA (Fiedler et al., 2002; Lüdtke, 2012).

Moreover, the accumulation of resonance-stabilized enamines has been a paradigm in the field of ThDP-dependent enzyme research. Several studies have now challenged this well- 
recognized feature through theoretical approaches as well as experimental methods. Amara and colleagues employed computational methods including hybrid quantum-mechanical $(\mathrm{QM}) /$ molecular mechanical (MM) to study one ThDP enzyme pyruvate:ferredoxin oxidoreductase (PFOR) and indicated that the enamine intermediate doesn't occur in the reaction pathway (Amara et al., 2008). Another study conducted by Meyer and colleagues (Meyer et al., 2012) suggested that the HEThDP intermediate captured in LpPOX at $1.1 \AA$ could be interpreted as a mixture of ketone and carbanion in a tautomeric equilibrium. These results are consistent with the crystal structures of the observed DHEThDP intermediates shown in this thesis as well as data from our previous colleague (Lüdtke, 2012), supporting the proposal that the enamine intermediate is not universal in the catalytic pathway of thiamine-dependent enzymes.

\subsubsection{Kinetical studies on the donor and acceptor half reactions of $h$ TK with non- phosphorylated substrates}

As shown in Fig 40, single-mixing stopped-flow analysis of AP depletion reveals that the reactivity of the five-carbon xylulose is 15 times faster than that of the six-carbon fructose. The mechanism for this behaviour might be as followings. The physiological ketose F6P and the non-physiological sugars fructose and xylulose discussed above are predominately occurring as the ring-cyclic forms under physiological conditions. Closer inspection on the chemical composition of the two non-phosphorylated sugars (Barclay et al., 2012; Bubb, 2003) reveals a relatively higher distribution of the reactive keto form (xylulose: $20 \%$, fructose: 0.5 $\%$, Fig 58) for the five-carbon xylulose relative to the six-carbon counterpart. This might partially explains the 15-fold increased reaction velocity for xylulose over fructose. Since our structural results presented before only indicate the DHEThDP intermediate and the cleavedoff substrates, it is reasonable to raise the question that which conformational form the enzyme prefers to bind and whether the enzyme carries out the ring-opening after binding of the cyclic form. To answer this question, the native reaction of transketolase for the formation of donor F6P-ThDP adduct seems to be a better system compared to the non-physiological reaction. Previous studies on the native donor substrate F6P in aqueous solution indicates a reaction velocity of approx. $20 \mathrm{~s}^{-1}$ at $40{ }^{\circ} \mathrm{C}$ and $\mathrm{pH} 7.5$ for the ring-opening. The rate constants for the $\alpha$-anomeric and the $\beta$-anomeric forms are $18 \mathrm{~s}^{-1}$ and $21 \mathrm{~s}^{-1}$ respectively (Pierce et al., 1985). These are slower than the velocity for formation of covalent donor-ThDP adducts on $h \mathrm{TK}$ (80 $\mathrm{s}^{-1}$ ) performed at lower temperatures $\left(20{ }^{\circ} \mathrm{C}\right)$ (Fig 52), which strongly indicates that transketolase could bind and accelerate the ring-opening of the cyclic forms in situ. But so far no direct evidence in literature could answer the question that which cyclic form transketolase is prone to bind, either promiscuously for both anomers, or specifically one of these forms. 
Since the active sites of $h \mathrm{TK}$, or in general, the transketolase family, are comprised of a variety of polar amino acid residues such as serine, arginine and histidine as well as numerous water molecules, it is reasonable to assume that ring-opening could be potentially accomplished by general acid-base catalysis (Fig 58). Previous research revealed that ring-opening of the phosphorylated sugars is notably faster than that of the non-phosphorylated forms (Serianni et al., 1982), which would also help to explain the extremely slow reaction for the formation of DHEThDP intermediate using the non-phosphorylated sugars.

Interestingly, both cyclic and acyclic form of the acceptor R5P were captured at one active site of E.coli transketolase with only acyclic form at another active site (Asztalos et al., 2007). Further investigation of the binding cavity suggests that the phosphate moiety of R5P itself mostly likely participating in the ring-opening with the assistance of three water molecules in a concerted manner (Tittmann, 2014). This finding strongly supports the substrate-assisted ring-opening as proof-of-principle.

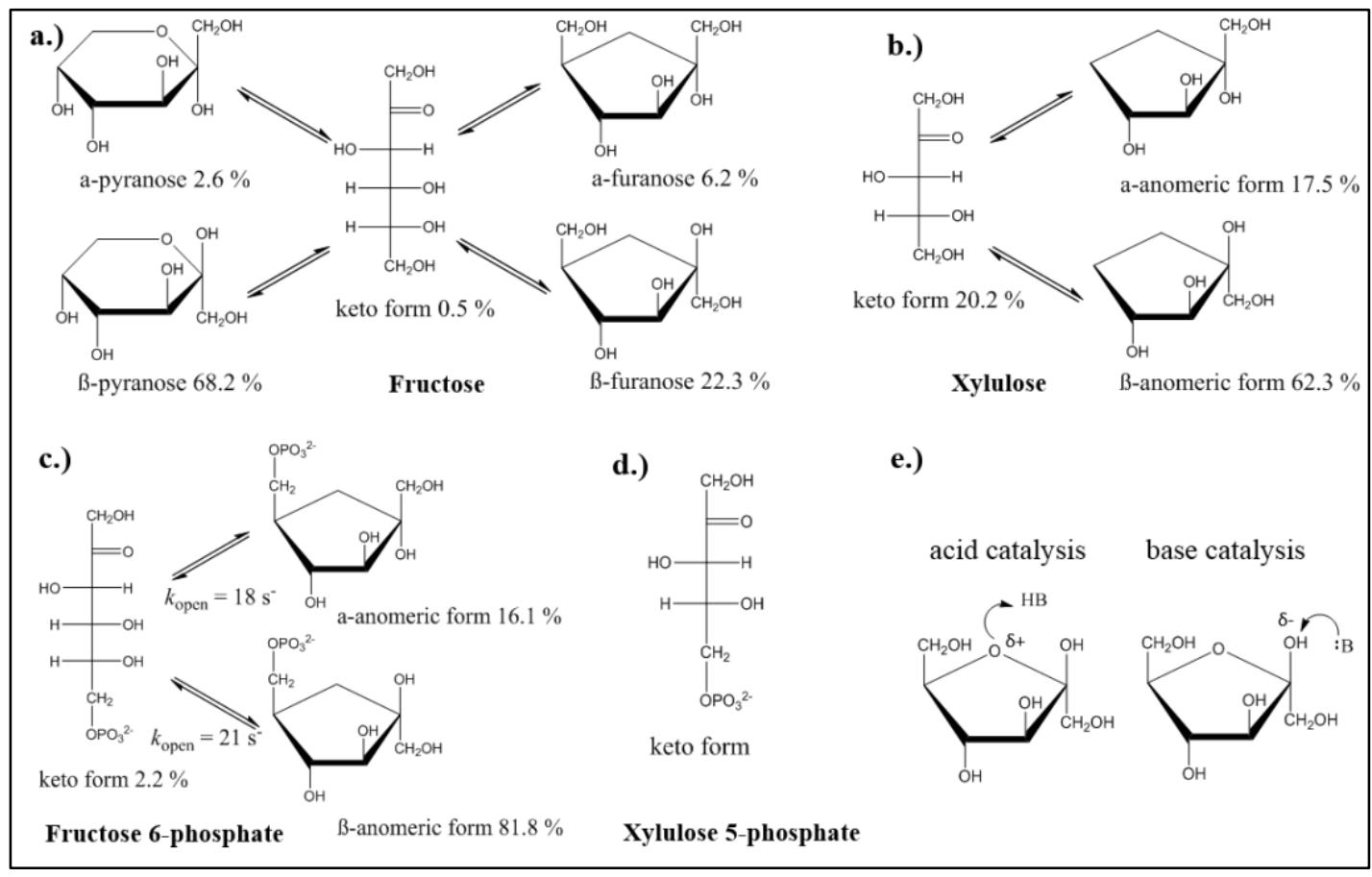

Fig 58: Conformational equilibrium compositions of sugars in aqueous solution. The chemical structures of possible conformations of fructose, xylulose, F6P and X5P are depicted in Fischer or Haw orth projections well as the relative distributions at given conditions. a.) fructose at $35^{\circ} \mathrm{C}$ (Barclay et al., 2012). b.) xylulose at $35{ }^{\circ} \mathrm{C}$. (Bubb, 2003) c.) F6P at $6{ }^{\circ} \mathrm{C}$ and pH 4.5. (Pierce et al., 1985) d.) $\mathrm{X} 5 \mathrm{P}$ exclusively in keto form. e.) mechanism of acid/base-catalysed ring-opening of the $\beta$-furanose from fructose. 


\subsubsection{Potential application of transketolase using fructose in the field of biocatalysis}

Biocatalysis is a practical and environment friendly approach compared to the traditional metallo- and organo-catalysis and has achieved unprecedented development over the past ten years (Bornscheuer et al., 2012). A variety of biological enzymes have shown their potential to achieve high stereoselectivities, including transketolase. Compared with the in vivo reaction, the TK-catalysed reaction has been made more generally applicable by using HPA as donor substrate since HPA renders the reaction virtually irreversible by releasing carbon dioxide as by-product (Turner, 2000). One example for a multi-enzyme approach for the synthesis of D-xylulose 5-phosphate has been developed and gives an overall yield of $82 \%$ and can be applied on a gram scale (Shaeri et al., 2008). Payongsri and co-workers made use of directed evolution and generated TK active-site mutants which could take aromatic aldehydes as acceptor substrates but still use HPA as donor (Payongsri et al., 2015).

Based on the crystallographic structures and the sequential stopped-flow kinetic analysis, we have discovered that the non-phosphorylated fructose and xylulose could serve as donor substrates to form the DHEThDP intermediate which is capable to react with acceptor R5P. The structural features and reactivity of obtained intermediates are quite identical with that of HPA, suggesting the potential use of these sugars as a replacement donor for HPA in the field of TK-mediated biocatalysis. Although HPA is commercially available and relatively cheaper compared with physiological substrates F6P and X5P, the use of the non-phosphorylated sugar fructose (xylulose is expensive) would greatly reduce the cost especially on large-scale synthesis. People may argue that the reaction efficiency $\left(k_{\mathrm{cat}} / \mathrm{K}_{\mathrm{m}}\right)$ with fructose is relatively lower than HPA and requires longer reaction time, but considering the advantage of extremely low cost, fructose still overwhelms HPA for industrial purposes. One aspect which needs further investigation is the feasibility of fructose for TK active-site mutants designed for using aromatic aldehydes.

Villafranca and colleagues used non-phosphorylated acceptor sugar D-ribose and found that TK could also take D-ribose as acceptor, further studies of the reaction between sedoheptulose and ribose demonstrated the exchange of a $\mathrm{C} 2$-fragment, suggesting an analogous reaction with non-phosphorylated donors and acceptors to that of phosphorylated substrates (Villafranca and Axelrod, 1971). This study along with our results indicate that both donor and acceptor non-phosphorylated sugars are feasible for TK-mediated biocatalysis. 


\subsubsection{Binding energy and enzyme catalysis}

The most important aspect in enzyme catalysis is that as catalysts enzymes significantly reduce the activation energy for the reaction. Much of the energy required to lower the activation energy is derived from weak, noncovalent interactions between substrate and enzyme. Formation of each weak interaction in the ES complex is accompanied by the release of small amount of energy. This energy derived from enzyme substrate interaction is called binding energy (Hansen and Raines, 1990). In the study of ScOMPDC, up to 80000-fold reaction efficiency for the truncated substrate lacking the phosphodianion was contributed to a binding energy of $6.6 \mathrm{kcal} / \mathrm{mol}$ (Amyes et al., 2005). X-ray crystallography structures of ScOMPDC suggested an open conformation for the resting state while a closed conformation in complex with a substrate analogue (Goldman et al., 2014). Similar kinetic and structural features have been proposed for other enzymes, such as triose phosphate isomerase (TIM) (Malabanan et al., 2011) and glycerol 3-phosphate dehydrogenase (GPDH) (Tsang et al., 2008).

Our results of phosphite dianion binding are reminiscent of the concept of binding energy. When exogenous phosphite enters the active site of enzyme, the interaction of the ligand with the protein renders Ser345 undergo a conformational change and slightly opens up the substrate entrance. Moreover, mediated by concerted hydrogen bonding network, binding of the phosphite dianion at a remote position from the reacting center induces the cofactor ThDP into a more reactive state with high flexibility of the $\mathrm{C} 2$ atom. The higher atomic $\mathrm{B}$-factors for the whole ThDP cofactor as well as for the $\mathrm{C} 2$ reacting center suggest that binding of phosphite enhances the reactivity, thus accelerating the formation of the DHEThDP intermediate. A similar mechanism could be envisaged for the physiological substrates X5P, F6P and S7P, the phosphodanion moiety serves as anchor and interacts with the enzyme to lower the binding energy and activate the cofactor before the nucleophilic reaction takes place. This process proceeds too transiently to be observed with native substrates but luckily the use of one piece of substrates helps the capture of this snapshot in catalysis.

\subsection{Intrinsic dynamics in human and E.coli transketolase}

Histidine residues are present at the active sites of numerous enzymes and have been generally accepted as acid-base catalysts. The $\mathrm{pKa}$ of the imidazolium ion is around neural, which allows histidine residues to act as both proton donor and acceptor. Other than the $\mathrm{p} K \mathrm{a}$ value, the geometric shape of histidine permits ring flipping of the imidazole function, which acts as a vehicle for proton transfer to facilitate the breakdown of substrates in serine protease (Rebek, 1990). In E.coli TK, His 473 side chain was proposed to flip over $180^{\circ}$ and two alternative conformations were observed (Neumann and Tittmann, 2014). In this PhD study, Asp469, 
which interacts with His473 though a water molecule is deeply studied. The His473 in both crystal structures of EcTK D469N and D469A adopts two alternative conformations, which is similar to that of the wild type enzyme (Fig 47). In addition, His473 in the apo-D469N crystal structure $\left(1.20 \AA, \mathrm{R}_{\text {work }}=12.35 \%, \mathrm{R}_{\text {free }}=14.49 \%\right.$, Fig 67) also exhibits two conformations, leading to the conclusion that the flipping of His473 is an intrinsic behavior and not influenced by the cofactor binding. Substrate soaking experiments with physiological substrate F6P failed for both variants due to their inability to bind F6P, which is revealed by stopped-flow experiments (Fig 68). The CD-based $\mathrm{pH}$ titration measurement reveal that deviated $\mathrm{pKa}$ values for the D469N and D469A variants to that of the wild type, indicating that Asp469 participates in the process of $([\mathrm{AP}]+[\mathrm{IP}]) /\left[\mathrm{APH}^{+}\right]$equilibrium (Fig 48). In addition, stopped-flow measurements with both physiological substrate F6P and non-native substrate HPA (Fig 49, 68) indicates potential application of enzyme mutation at Asp469 to change the enzyme's substrate specificity from phosphorylated sugars to non-phosphorylated substrates. Smith and colleagues have discovered that EcTK D469X single mutant has the ability to accept aromatic acceptors, in particular benzaldehyde for which the wild type enzyme shows no activity (Smith et al., 2008).

In the last part of this $\mathrm{PhD}$ thesis, a preliminary study of the "dynamic hotspots" in human $\mathrm{TK}$ is conducted. Three active sites variants along the proposed hydrogen bonding pathway are generated and the crystal structures in complex with native substrate F6P are determined. Different active site architectures are observed within these crystal structures. For example, $h \mathrm{TK}-\mathrm{S} 427 \mathrm{~A}$ and $h \mathrm{TK}-\mathrm{H} 77 \mathrm{~A}$ only show one conformation for the related four residues, while for $h$ TK-Q428A two alternative conformations are observed for residues Thr122 and Gly123 (Fig 51). Pre-steady-state measurements using stopped-flow spectroscopy have revealed reduced catalytic activity for these mutants (Fig 52, 4\% for H77A and 40\% for Q428A compared to wild type). However, since Gln428 and His 77 are also involved in the substrate or cofactor binding, therefore the intrinsic dynamics of the active site is not the sole explanation for the reduced activity. 


\section{Summary and Conclusion}

Thiamine diphosphate (ThDP) dependent enzymes play central roles in the metabolism of biological systems and these enzymes possess powerful ability to catalyse the formation and breakage of carbon-carbon bonds. A variety of reactions are involved in this cofactor mediated enzymatic reactions such as decarboxylation (in pyruvate decarboxylase (PDC)), carboligation (in transketolase (TK) or acetolactate synthase (AHAS)) and oxidative transformations (in pyruvate dehydrogenase complex (PDHc) or pyruvate oxidase (POX)) (Kluger and Tittmann, 2008). The catalytic mechanism of ThDP-dependent enzymes has been extensively studied over the last 50 years after Breslow (Breslow, 1957) discovered in 1957 that the deprotonation at the $\mathrm{C} 2$ of the thiazolium ring is the first reaction step. Among these enzymes, transketolase is a very representative example which catalyses the reversible transfer of a C2-unit from ketose phosphates to the $\mathrm{C} 1$ position of aldose phosphates (Mitschke et al., 2010).

Previous colleagues in our group have solved the crystal structure of the genius covalent intermediates at ultra-high resolution and characterised a scissile bond elongation and out-ofplane distortion to be essential in the catalytic process of TK-reactions. This doctoral thesis is a follow-up work based on these extraordinary findings and presents a more comprehensive study of transketolase which involves the participation of a low barrier hydrogen bond (LBHB). As illustrated before, a LBHB is a special type of hydrogen bond when the $\mathrm{pKa}$ values of the H-bond donor and acceptor are matched. This leads to the formation of a short and strong hydrogen bond (bond length is around $2.55 \AA$ ). After careful reanalysis of the previously obtained sub-angstrom resolution crystal structures, a LBHB with a distance of $2.56 \AA$ is unexpectedly observed between a conserved glutamate (Glu366 in $h \mathrm{TK}$ ) and a neighbouring glutamate (Glu160) from the adjacent subunit. A hydrogen atom is unambiguously modelled almost at the equidistant position between the $\mathrm{H}$-bond donor and acceptor. More interestingly, the observed LBHB locates in a proton tunnel which can synchronize the two active sites by several acidic amino acid residues and water molecules. This proton wire was first proposed in the $\mathrm{E} 1$ component of pyruvate dehydrogenase complex (PDHc) and received wide acceptance among ThDP-dependent enzyme family.

In order to investigate the catalytic function of the LBHB in $h \mathrm{TK}$, a single mutation variant E160Q was generated and the crystal structure with donor substrate F6P was determined at ultra-high resolution. Instead of the LBHB, an ordinary hydrogen bond (bond length around $2.80 \AA$ ) is observed with a well-defined protonation state for the amide function. The crystal structure of this variant at ground state also reveals the existence of an ordinary hydrogen bond, although the protons on the amide group can't be precisely determined. Furthermore, 
the F6P-ThDP covalent intermediate in the crystal structure of $h \mathrm{TK}-\mathrm{E} 160 \mathrm{Q}$ exhibits identical scissile bond elongation and out-of-plane distortion as that in the wild type, suggesting a similar energy profile for both proteins. Steady-state kinetic analysis reveals that the enzymatic activity $\left(k_{\text {cat }}\right)$ of the wild type $\left(2.79 \pm 0.06 \mathrm{~s}^{-}\right)$is five times faster than the E160Q mutant $(0.54$ $\left.\pm 0.01 \mathrm{~s}^{-}\right)$, but both proteins exhibit similar substrate affinity for the donor substrate X5P $\left(K_{\mathrm{m}}\right.$ for wild type and E160Q is $73.9 \pm 6.7 \mathrm{mM}$ and $78.0 \pm 4.8 \mathrm{mM}$, respectively). Pre-steady-state kinetic analysis by stopped-flow technique revealed that the donor half reaction of $h \mathrm{TK}$ wild type exhibits positive cooperativity $(n=1.56 \pm 0.26$ for AP depletion and $1.49 \pm 0.43$ for IP formation) and is two times faster than that of the E160Q variant which shows no cooperativity $\left(k_{\mathrm{obs}}{ }^{\max }\right.$ of wild type and E160Q for AP depletion is $9.06 \pm 0.72 \mathrm{~s}^{-}$and $5.91 \pm 0.43 \mathrm{~s}^{-}$, respectively). Those structural and kinetic results indicate that the LBHB in $h$ TK plays a role in enzymatic efficiency and positive cooperativity for the two active sites. A Circular Dichroism (CD) based $\mathrm{pH}$ titration experiment reveals the $\mathrm{pKa}$ difference between human TK wild type and E160Q for the $[\mathrm{AP}]+[\mathrm{IP}]) /\left[\mathrm{APH}^{+}\right]$equilibrium is around $1.5 \mathrm{pH}$ units which corresponds to $2.01 \pm 0.01 \mathrm{kcal} / \mathrm{mol}$. This experimental result would revise the current knowledge about the energy profile of LBHBs which is obtained from computational calculation.

In the next part of this thesis, crystal structures of $h \mathrm{TK}$ with non-phosphorylated sugars xylulose and fructose lead to the accumulation of the post-cleavage DHEThDP intermediate, which is further confirmed by the H1-NMR and UV-Vis spectroscopy. The leaving groups Dglyceraldehyde and D-erythrose are also captured within the crystal structures and are found at the entrance of the active site. Stopped-flow analysis of the non-phosphorylated sugars with $h \mathrm{TK}$ reveals a very slow reaction process. The depletion of AP form, the formation of IP and enamine form have shown identical reaction velocity at the same substrate concentrations, indicating a same molecular origin for those different forms. Five-carbon sugar xylulose shows a 15-fold increased reaction velocity relative to the six-carbon counterpart fructose. This is consistent with the literature reported conformational equilibrium compositions of sugars that xylulose has $20 \%$ active keto form while fructose only shows $0.5 \%$. The reactivity of the accumulated DHEThDP intermediate is confirmed by a sequential stopped-flow experiment using the native substrate R5P as the acceptor. Positive cooperativity $(n=1.67 \pm 0.27)$ is observed for the acceptor half reaction with $\mathrm{V}_{\max }$ of $92.3 \pm 6.1 \mathrm{~s}^{-}$and $K \mathrm{~s}_{\text {app }}$ of $0.71 \pm 0.14 \mathrm{mM}$. Supplement of exogenous phosphite dianion to human TK is shown to have activation function for the donor half reaction using the non-phosphorylated sugars. A 2-3 fold reaction acceleration of saturation phosphite concentration is observed for both 5-carbon and 6-carbon sugars. X-ray crystal structure in complex with phosphite dianion shows an induced 
conformation change of Ser345 which potentially lowers the binding energy thus enhances the reaction. In addition, the phosphite dianion is bound at a remote but same position where phosphate group of native substrates binds, revealing the potential role for the native substrates' phosphate group.

In the last part of this thesis, intrinsic dynamics of several catalytic residues have been studied. Ring flipping of a histidine side chain is observed in E.coli transketolase and several related enzyme mutants are studied. In human TK, a structural plasticity of the active site has been observed and site-directed mutagenesis studies give a preliminary insight into this intrinsic dynamics.

In general, this doctoral thesis has presented a comprehensive study of a LBHB in transketolase and have characterised a DHEThDP intermediate obtained from nonphosphorylated sugars. Those results indicate that although ThDP-dependent transketolase has been extensively studied over the last 30 years, a lot of knowledge for this enzyme as well as the whole ThDP-dependent enzyme family are still missing. More work from both experimental and computational aspects should be conducted in the future. 


\section{Appendix}

Table 6: X-ray statistics for the crystal structures of human transketolase E160Q ground state, E160Q in covalent complex with donor substrate fructose 6-phosphate (F6P) and E366Q in non-covalent complex with donor substrate xylulose 5-phosphate (X5P).

\begin{tabular}{|c|c|c|c|}
\hline & $\begin{array}{l}h \text { TK-E160Q } \\
\text { ground state }\end{array}$ & $\begin{array}{c}h \text { TK-E160Q } \\
+ \text { F6P }\end{array}$ & $\begin{array}{r}h \text { TK-E366 } \\
+ \text { X5P } \\
\end{array}$ \\
\hline \multicolumn{4}{|l|}{ Data collection } \\
\hline Wavelength $(\AA)$ & 0.97625 & 0.97625 & 0.97625 \\
\hline Space group & $\mathrm{C} 2$ & $\mathrm{C} 2$ & $\mathrm{C} 2$ \\
\hline \multicolumn{4}{|l|}{ Cell dimensions } \\
\hline$a(\AA)$ & 115.7 & 114.1 & 114.7 \\
\hline$b(\AA)$ & 85.5 & 86.1 & 86.2 \\
\hline c $(\AA)$ & 73.6 & 73.2 & 73.4 \\
\hline$\alpha\left(^{\circ}\right)$ & 90.0 & 90.0 & 90.0 \\
\hline$\beta\left(^{\circ}\right)$ & 127.8 & 125.2 & 125.6 \\
\hline$\gamma\left({ }^{\circ}\right)$ & 90 & 90.0 & 90.0 \\
\hline
\end{tabular}

$\begin{array}{cccc}\text { Resolution range }(\AA) & 50-1.04 & 25-1.08 & 25-1.5 \\ \text { No. of reflections } & (1.11-1.04) & (1.15-1.08) & (1.6-1.5) \\ \text { No. of unique } & 1692285(280303) & 1383209(232616) & 304158(53182) \\ \text { reflections } & 263585(47044) & 238255(39930) & 90973(15949) \\ \text { Completeness }(\%) & 97.3(93.5) & 96.4(94.4) & 97.7(97.8) \\ \text { I/sigma (I) } & 14.73(2.00) & 13.94(2.43) & 31.48(2.44) \\ R_{\text {meas }}(\%) & 5.8(94.6) & 5.7(74.3) & 2.1(74.5) \\ \mathrm{Redundancy} & 6.42(5.96) & 5.81(5.83) & 3.3(3.3) \\ \mathrm{CC}_{1 / 2}(\%) & 99.9(72.7) & 99.9(94.4) & 100(79.6)\end{array}$




\begin{tabular}{|c|c|c|c|}
\hline & $\begin{array}{l}h \text { TK-E160Q } \\
\text { ground state }\end{array}$ & $\begin{array}{c}h \text { TK-E160Q } \\
+ \text { F6P } \\
\end{array}$ & $\begin{array}{c}h \text { TK-E366Q } \\
+ \text { X5P } \\
\end{array}$ \\
\hline \multicolumn{4}{|l|}{ Refinement } \\
\hline Resolution range ( $\mathrm{A})$ & $50-1.04$ & $25-1.08$ & $25-1.5$ \\
\hline $\mathrm{R}_{\text {work }} / \mathrm{R}_{\text {free }}(\%)$ & $11.26 / 13.14$ & $11.12 / 13.05$ & $15.83 / 18.99$ \\
\hline \multicolumn{4}{|l|}{ Number of atoms } \\
\hline Protein & 5748 & 5860 & 4834 \\
\hline Ligands & 14 & 95 & 51 \\
\hline Water & 681 & 670 & 436 \\
\hline B-factor Protein & 14.64 & 15.31 & 39.30 \\
\hline B-factor Ligands & 22.33 & 26.14 & 38.80 \\
\hline B-factor Water & 27.31 & 27.95 & 45.70 \\
\hline \multicolumn{4}{|c|}{ Deviations from ideals (r.m.s.d.) } \\
\hline Bond distances $(\AA)$ & 0.010 & 0.011 & 0.011 \\
\hline Bond angles $\left({ }^{\circ}\right)$ & 1.476 & 1.83 & 1.38 \\
\hline Dihedrals $\left({ }^{\circ}\right)$ & 10.53 & 11.25 & 13.30 \\
\hline \multicolumn{4}{|l|}{ Ramachandran plot } \\
\hline $\begin{array}{l}\text { Favoured regions } \\
\qquad(\%)\end{array}$ & 98 & 98 & 98 \\
\hline Allowed regions (\%) & 2 & 2 & 2 \\
\hline Outlier regions (\%) & 0 & 0 & 0 \\
\hline $\begin{array}{l}\text { B-factor from Wilson } \\
\qquad \text { Plot }\left(\mathrm{A}^{2}\right)\end{array}$ & 10.74 & 11.37 & 27.08 \\
\hline
\end{tabular}


Table 7: X-ray statistics for the crystal structures of human transketolase wild type in complex with non-phosphorylated sugars xylulose and fructose as well as human transketolase wild type with activating phosphite dianion.

\begin{tabular}{ccc}
$h$ TK-wt & $h$ TK-wt & $h$ TK-wt \\
+ xylulose & + fructose & + phosphite \\
\hline
\end{tabular}

\section{Data collection}

Wavelength $(\AA)$

Space group

Cell dimensions

$$
\begin{aligned}
& a(\AA) \\
& b(\AA) \\
& c(\AA) \\
& \alpha\left(^{\circ}\right) \\
& \beta\left(^{\circ}\right) \\
& \gamma\left({ }^{\circ}\right)
\end{aligned}
$$

Resolution range $(\AA)$

No. of reflections

No. of unique reflections

Completeness (\%)

I/sigma (I)

$R_{\text {meas }}(\%)$

Redundancy

$\mathrm{CC}_{1 / 2}(\%)$
0.82656

P21

73.5

86.5

92.7

90.0

93.8

90.0

$50-1.5$

(1.6 - 1.5)

1251117 (182855)

$223490(31820)$

$98.9(98.1)$

$16.96(2.53)$

$6.6(91.1)$

$6.8(6.7)$

$99.9(81.5)$
0.855

P21

$\mathrm{C} 2$

73.4

86.4

92.5

90.0

94.0

90.0

114.5

86.4

73.4

90.0

125.5

90.0

$25-1.5$

$25-1.48$

(1.6 - 1.5)

(1.60 - 1.48)

597042 (105119)

313356 (94543)

$342211(59465) \quad 65740(19699)$

$94.2(92.9)$

$97.4(97.7)$

$5.29(1.38)$

$28.14(2.70)$

$10.8(65.1)$

$2.5(67.1)$

$1.7(1.7)$

$3.3(3.2)$

$99.2(61.5)$

$99.9(75.2)$ 


\begin{tabular}{|c|c|c|c|}
\hline & $\begin{array}{c}\text { hTK-wt } \\
+ \text { xylulose } \\
\end{array}$ & $\begin{array}{c}h \text { TK-wt } \\
+ \text { fructose } \\
\end{array}$ & $\begin{aligned} & h \text { TK-wt } \\
&+ \text { phosphite } \\
&\end{aligned}$ \\
\hline \multicolumn{4}{|l|}{ Refinement } \\
\hline Resolution range ( $\mathrm{A})$ & $50-1.5$ & $25-1.5$ & $25-1.48$ \\
\hline $\mathrm{R}_{\text {work }} / \mathrm{R}_{\text {free }}(\%)$ & $14.42 / 17.76$ & $15.69 / 18.10$ & $15.04 / 17.53$ \\
\hline \multicolumn{4}{|l|}{ Number of atoms } \\
\hline Protein & 11021 & 10652 & 5320 \\
\hline Ligands & 132 & 120 & 53 \\
\hline Water & 1130 & 1232 & 632 \\
\hline B-factor Protein & 16.25 & 18.32 & 17.52 \\
\hline B-factor Ligands & 18.35 & 19.63 & 08.98 \\
\hline B-factor Water & 20.35 & 22.12 & 22.56 \\
\hline \multicolumn{4}{|c|}{ Deviations from ideals (r.m.s.d.) } \\
\hline Bond distances $(\AA)$ & 0.016 & 0.009 & 0.006 \\
\hline Bond angles $\left({ }^{\circ}\right)$ & 1.648 & 1.281 & 1.101 \\
\hline Dihedrals $\left(^{\circ}\right)$ & 13.604 & 12.909 & 12.930 \\
\hline \multicolumn{4}{|l|}{ Ramachandran plot } \\
\hline $\begin{array}{l}\text { Favoured regions } \\
\qquad(\%)\end{array}$ & 97.39 & 97.16 & 97.82 \\
\hline Allowed regions (\%) & 2.45 & 2.68 & 2.02 \\
\hline Outlier regions (\%) & 0.15 & 0.15 & 0.16 \\
\hline $\begin{array}{c}\text { B-factor from Wilson } \\
\text { Plot }\left(\mathrm{A}^{2}\right)\end{array}$ & 28.65 & 23.68 & 29.86 \\
\hline
\end{tabular}


Table 8: X-ray statistics for the crystal structures of human transketolase active site variants Q428A, S427A and H77A in complex with donor substrate fructose 6-phosphate (F6P).

\begin{tabular}{ccc}
$h \mathrm{TK}-\mathrm{Q} 428 \mathrm{~A}$ & $h \mathrm{TK}-\mathrm{S427A}$ & $h \mathrm{TK}-\mathrm{H} 77 \mathrm{~A}$ \\
$+\mathrm{F} 6 \mathrm{P}$ & $+\mathrm{F} 6 \mathrm{P}$ & + F6P \\
\hline
\end{tabular}

\section{Data collection}

Wavelength (̊̊)

0.855

0.82656

0.855

Space group

P21

$\mathrm{P} 21$

$\mathrm{C} 2$

Cell dimensions

$\begin{array}{cccc}\mathrm{a}(\AA) & 73.1 & 73.1 & 114.1 \\ \mathrm{~b}(\AA) & 85.9 & 95.9 & 86.2 \\ \mathrm{c}(\AA) & 92.8 & 73.4 \\ \alpha\left(^{\circ}\right) & 90.0 & 90.0 & 90.0 \\ \beta\left(^{\circ}\right) & 94.3 & 94.4 & 125.3 \\ \gamma\left({ }^{\circ}\right) & 90.0 & 90.0 & 90.0\end{array}$

Resolution range $(\AA)$

$50-1.5$

$50-1.25$

$50-1.9$

(1.6 - 1.5)

(1.37 - 1.25)

(2.0 - 1.9)

No. of reflections

520841 (330481)

1456399 (312845)

142956 (86063)

No. of unique reflections

85872 (55563)

336472 (74724)

19955 (12111)

Completeness (\%)

$91.4(87.3)$

$99.3(99.0)$

$95.4(94.2)$

I/sigma (I)

$7.0(1.7)$

$15.04(2.29)$

$3.95(0.95)$

$R_{\text {meas }}(\%)$

$9.0(75.2)$

$5.3(79.1)$

$20.6(101.9)$

Redundancy

$1.8(1.8)$

$2.3(2.2)$

$1.8(1.7)$

$\mathrm{CC}_{1 / 2}(\%)$

99.7 (75.2)

$100(81.8)$

$97.8(25.2)$ 


\section{$h \mathrm{TK}-\mathrm{Q} 428 \mathrm{~A}+\mathrm{F} 6 \mathrm{P} \quad h \mathrm{TK}-\mathrm{S} 427 \mathrm{~A}+\mathrm{F} 6 \mathrm{P} \quad h \mathrm{TK}-\mathrm{H} 77 \mathrm{~A}+\mathrm{F} 6 \mathrm{P}$}

\section{Refinement}

Resolution range $(\AA)$

$50-1.5$

$50-1.25$

$50-1.9$

$\mathrm{R}_{\text {work }} / \mathrm{R}_{\text {free }}(\%)$

$16.89 / 19.24$

$13.46 / 16.23$

$17.36 / 22.68$

Number of atoms

$\begin{array}{cccc}\text { Protein } & 10065 & 10135 & 5320 \\ \text { Ligands } & 120 & 132 & 52 \\ \text { Water } & 998 & 1032 & 556 \\ \text { B-factor Protein } & 21.35 & 20.12 & 45.65 \\ \text { B-factor Ligands } & 23.25 & 21.22 & 43.02 \\ \text { B-factor Water } & 30.98 & 30.21 & 48.74\end{array}$

Deviations from ideals (r.m.s.d.)

$\begin{array}{cccc}\text { Bond distances }(\AA) & 0.008 & 0.010 & 0.010 \\ \text { Bond angles }\left(^{\circ}\right) & 1.628 & 1.783 & 1.605 \\ \text { Dihedrals }\left(^{\circ}\right) & 13.963 & 14.101 & 14.525\end{array}$

Ramachandran plot

Favoured regions

$(\%)$

97.32

97.39

97.33

Allowed regions (\%)

2.53

2.45

2.52

Outlier regions (\%)

0.15

0.15

0.15

B-factor from Wilson

Plot $\left(\mathrm{A}^{2}\right)$

28.21

23.25

40.21 
Table 9: X-ray statistics for the crystal structures of E.coli transketolase active site variants D469N, D469A holo-enzyme in ground state and D469N apo-enzyme.

EcTK-D469N

\section{Data collection}

Wavelength $(\AA)$

Space group

Cell dimensions

$a(\AA)$
$b(\AA)$
$c(\AA)$
$\alpha\left(^{\circ}\right)$
$\beta\left(^{\circ}\right)$
$\gamma\left({ }^{\circ}\right)$
tion range $(\AA)$

Resolution range ( $\mathrm{A})$$$
25-1.06
$$$$
(1.10-1.06)
$$

102.2

133.1

90.0

90.0

90.0

$23900(1382988)$

No. of reflections

$6073900(1382988)$

1862797 (425446)

(1.20 - 1.15)

(1.2 - 1.1)

10349386

(1181962)

$$
\begin{aligned}
& \text { No. of unique } \\
& \text { reflections }
\end{aligned}
$$

Completeness (\%)

I/sigma (I)

$R_{\text {meas }}(\%)$

Redundancy

$\mathrm{CC}_{1 / 2}(\%)$
232454 (54163)

$95.7(92.5)$

$8.98(2.81)$

$10.2(39.7)$

$4.3(4.2)$

99.8 (77.9)
207057 (49648)

1819659 (214657)

$99.0(97.0)$

$98.1(97.3)$

$15.99(3.40)$

$11.36(4.48)$

$6.0(47.4)$

$31.6(60.7)$

$5.2(5.1)$

$8.5(8.3)$

$99.9(87.5)$

90.5 (73.3) 


\section{$E c$ TK-D469N}

\section{EcTK-D469N}

(apo)

\section{Refinement}

Resolution range $(\AA)$

$\mathrm{R}_{\text {work }} / \mathrm{R}_{\text {free }}(\%)$

$25-1.06$

$50-1.15$

$25-1.2$

Number of atoms

$\begin{array}{cccc}\text { Protein } & 12301 & 12166 & 10453 \\ \text { Ligands } & 243 & 210 & 65 \\ \text { Water } & 1862 & 1601 & 1657 \\ \text { B-factor Protein } & 10.65 & 16.63 & \text { n.d. } \\ \text { B-factor Ligands } & 14.32 & 23.21 & \text { n.d. } \\ \text { B-factor Water } & 20.24 & 25.98 & \text { n.d. }\end{array}$

Deviations from ideals (r.m.s.d.)

Bond distances $(\AA)$
Bond angles $\left(^{\circ}\right)$
Dihedrals $\left(^{\circ}\right)$
Ramachandran plot

Favoured regions

(\%)

98.40

98.33

98.55

Allowed regions (\%)

1.60

1.67

1.45

Outlier regions (\%)

0.00

0.00

0.00

B-factor from Wilson

Plot $\left(\mathrm{A}^{2}\right)$
10.35

20.74

26.32 


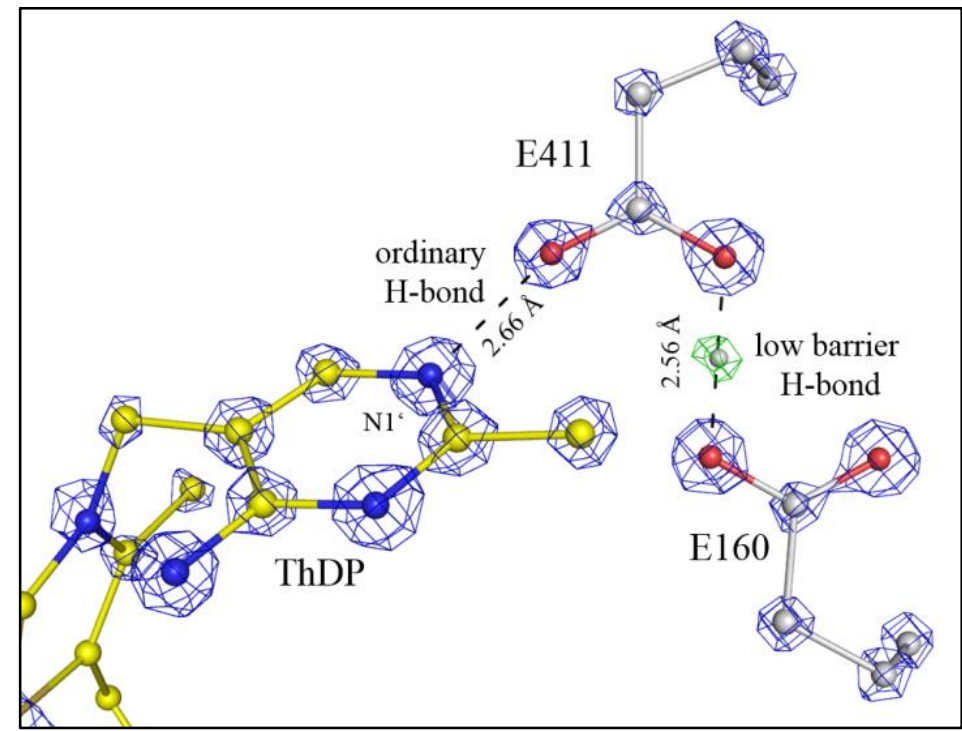

Fig 59: A possible low barrier hydrogen bond (LBHB) observed in the crystal structure of $E c$ TKD469N at a resolution of $1.06 \AA$. A LBHB is observed between Glu160 and Glu411 from neighbouring subunit with a hydrogen bond distance about $2.56 \AA$. The aminopyrimidine ring of ThDP, Glu160 and Glu411 are shown as ball and stick representation and surrounded by the calculated $2 \mathrm{mFo}-\mathrm{DFc}$ map (contoured at $4 \sigma$ level, blue). A hydrogen atom shown as sphere in grey could be observed at nearly equidistant position between E411 and E160 and is surrounded by a mFo-DFc map (contoured at $3 \sigma$ level, green). An ordinary hydrogen bond with distance of $2.66 \AA$ between N1' and E411 is also shown for comparison.
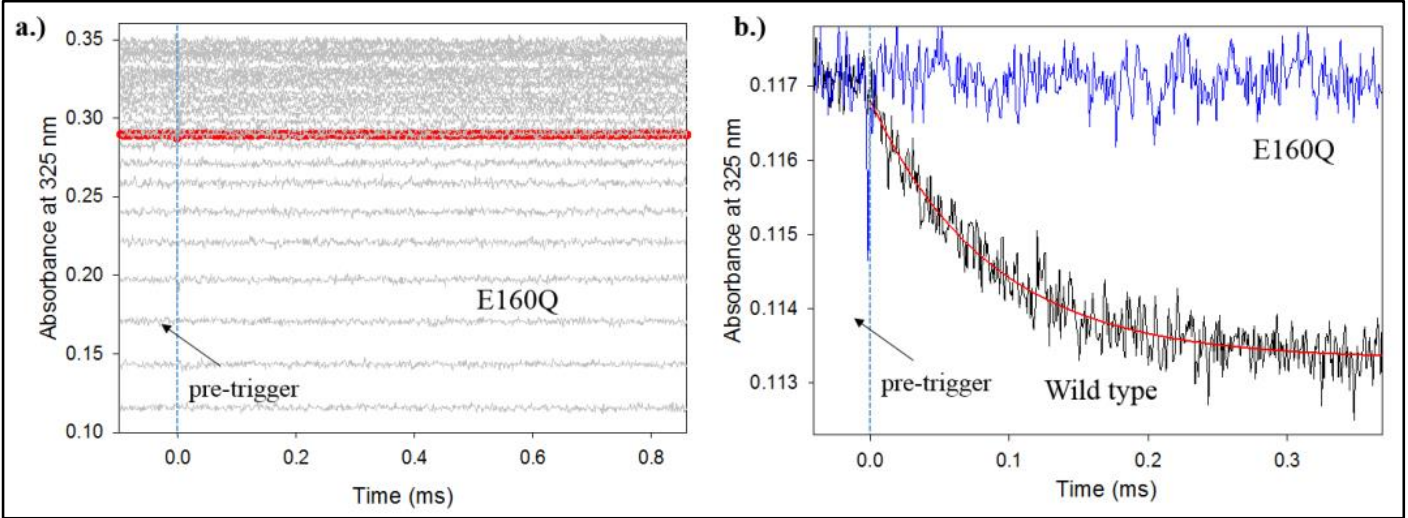

Fig 60: Temperature jump analysis of human TK E160Q. The AP tautomeric form of ThDP on $h$ TK-E160Q $\left(5 \mathrm{mg} / \mathrm{ml}\right.$ in $50 \mathrm{mM}$ glycylglycine, $5 \mathrm{mM} \mathrm{CaCl}_{2}, \mathrm{pH}$ 7.6) was studied by using the temperature jump relaxation technique with a jump of $10^{\circ} \mathrm{C}\left(12\right.$ to $\left.22^{\circ} \mathrm{C}\right)$ at $325 \mathrm{~nm}$. a.) Progress curve of 50 individual traces in one measurement. Please note that the increasing UV absorbance is caused by the precipitation of protein. The average of those 50 traces is shown in red. b.) Comparison of human TK-E160Q with wild type. The blue curve indicates that communication between the two active sites of transketolase is interrupted by the mutation. 


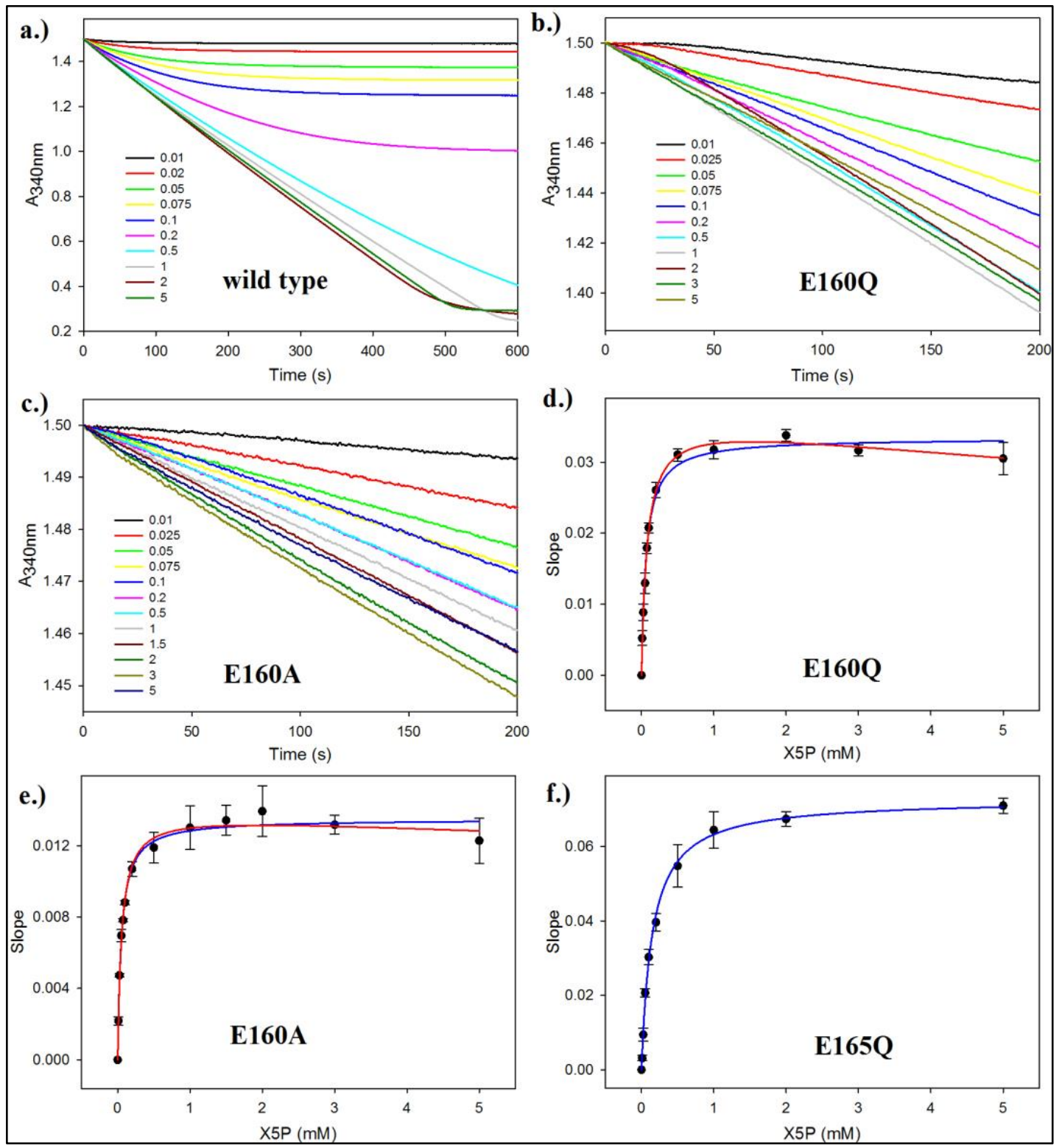

Fig 61: Steady-state kinetic analysis of human TK wild type and LBHB-related active site variants. a.) Progress curve of human TK wild type. The concentrations of X5P (in $\mathrm{mM}$ ) for each curve are shown. The concentration of R5P is kept at $5 \mathrm{mM}$ for all the measurements. b.) Progress curve of human TK E160Q. c.) Progress curve of human TK E160A. d.) Kinetic plot of the steady-state activity for $h \mathrm{TK}$ E160Q. Blue curve represents the fitting according to the Michaelis-Menten equation while the red curve represents the fitting according to the substrate inhibition equation. e.) Kinetic plot of the steady-state activity for $h$ TK E160A. f.) Kinetic plot of the steady-state activity for $h$ TK E165Q. 


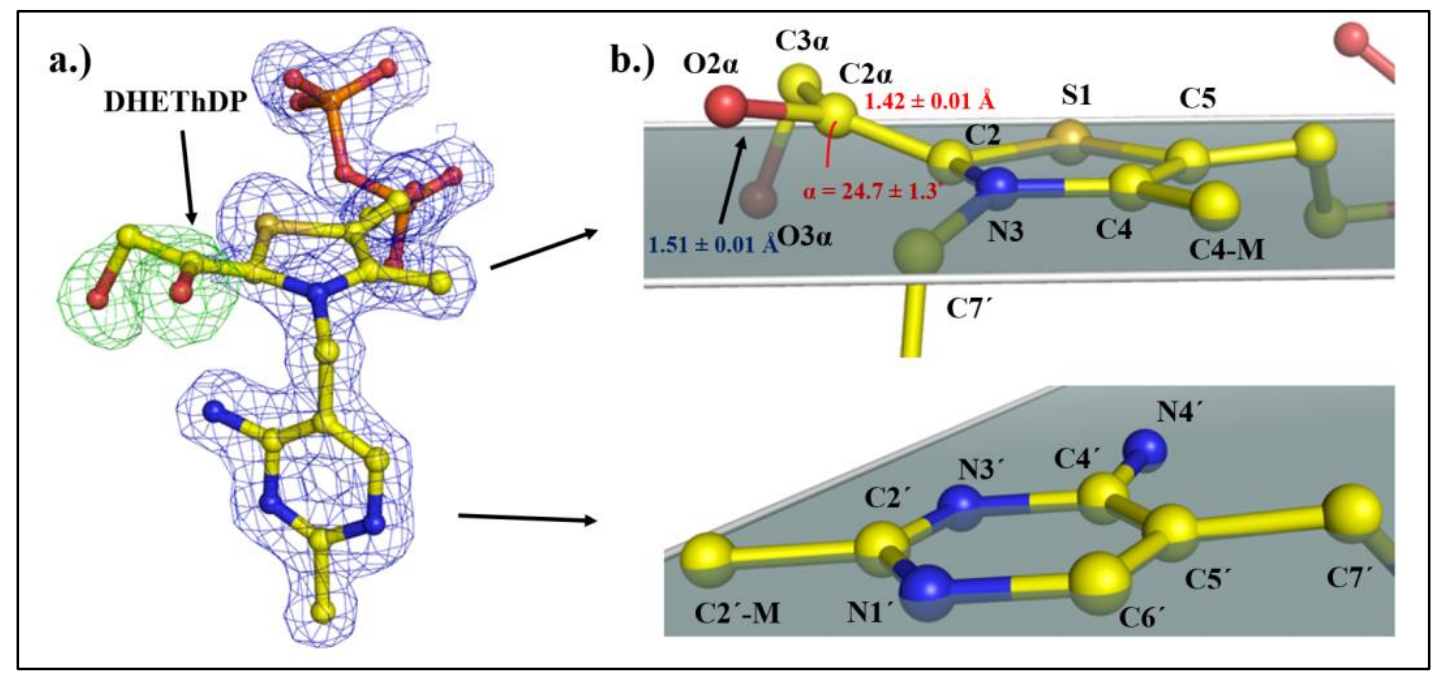

Fig 62: Detailed view of the carbanion-enamine intermediate trapped in $h \mathrm{TK}$ by using fructose at a resolution of $1.5 \AA$. a). The DHEThDP intermediate is shown in ball-stick representation with the $2 \mathrm{Fo}-\mathrm{Fc}$ map contoured at $1 \sigma$ level (blue) shown for ThDP moiety and the Fo-Fc map contoured at $3 \sigma$ level (green) shown for dihydroxyethyl moiety of DHEThDP intermediate. b). Close-up of the intermediate that highlights the deviations from planarity of the thiazolium moiety (top) and aminopyrimidine moiety (bottom) by shown with auxiliary planes. Selected atoms and bond lengths are labelled. 

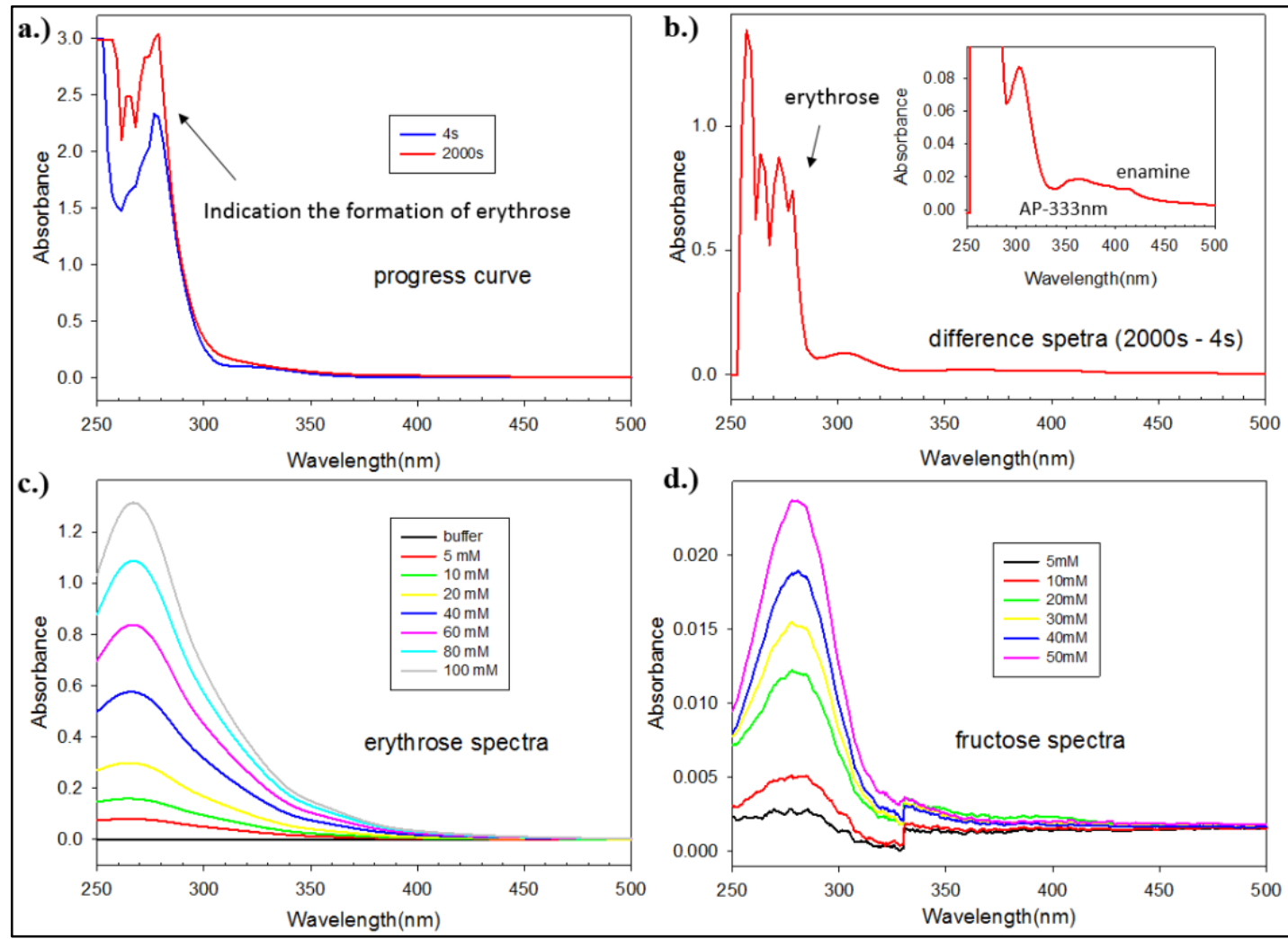

Fig 63: Photodiode array based stopped-flow analysis of human transketolase with nonphosphorylated sugar fructose. Single-mixing stopped-flow analysis (10 $\mathrm{mm}$ path length) for the reaction of $5 \mathrm{mg} / \mathrm{ml} h \mathrm{TK}, 5 \mathrm{mM} \mathrm{CaCl}_{2}, 50 \mathrm{mM}$ glycylglycine (pH 7.6) with $50 \mathrm{mM}$ fructose at $4{ }^{\circ} \mathrm{C}$ and measured by photodiode array. a.) Progress curve for the reaction after $4 \mathrm{~s}$ and $2000 \mathrm{~s}$ after mixing. b.) The difference spectra of the reaction after $2000 \mathrm{~s}$ and $4 \mathrm{~s}$. Please note that the huge absorbance increase at 270-280 nm indicates the accumulation of erythrose in the reaction mixture. Inset: zoom-in view of the spectra indicates the decrease of AP form and the increase of enamine form. c.) UV-Vis spectra of standard erythrose with strong maximal absorbance at 260-270 nm. d.) UV-Vis spectra of standard fructose with very small absorbance at $260-270 \mathrm{~nm}$ compared with erythrose. 


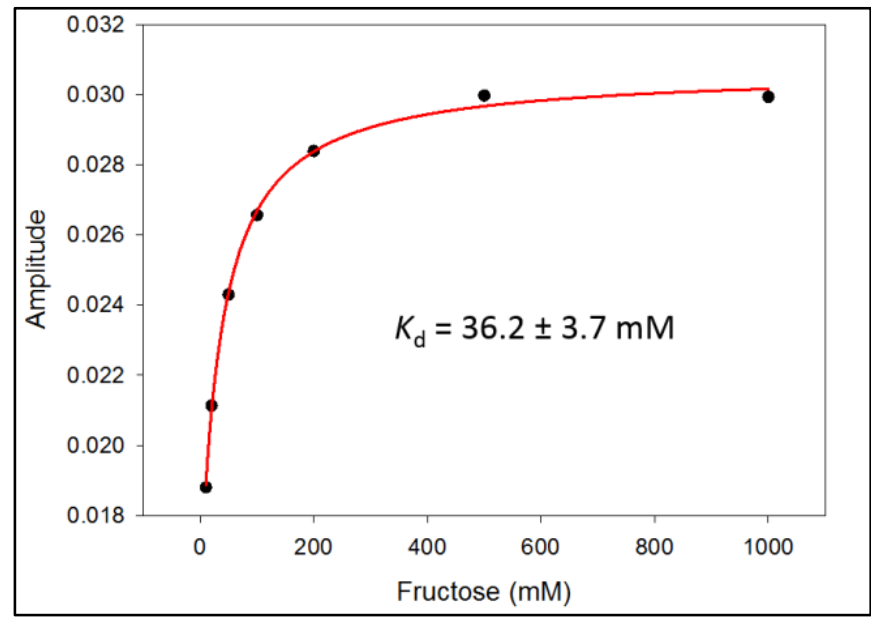

Fig 64: Single-mixing stopped-flow analysis of human TK with fructose. Plotting of absorbance amplitude against utilised fructose concentration reveals an apparent $K_{\mathrm{d}}$ value of $36.2 \pm 3.7 \mathrm{mM}$. Reaction condition refers to Fig 39.
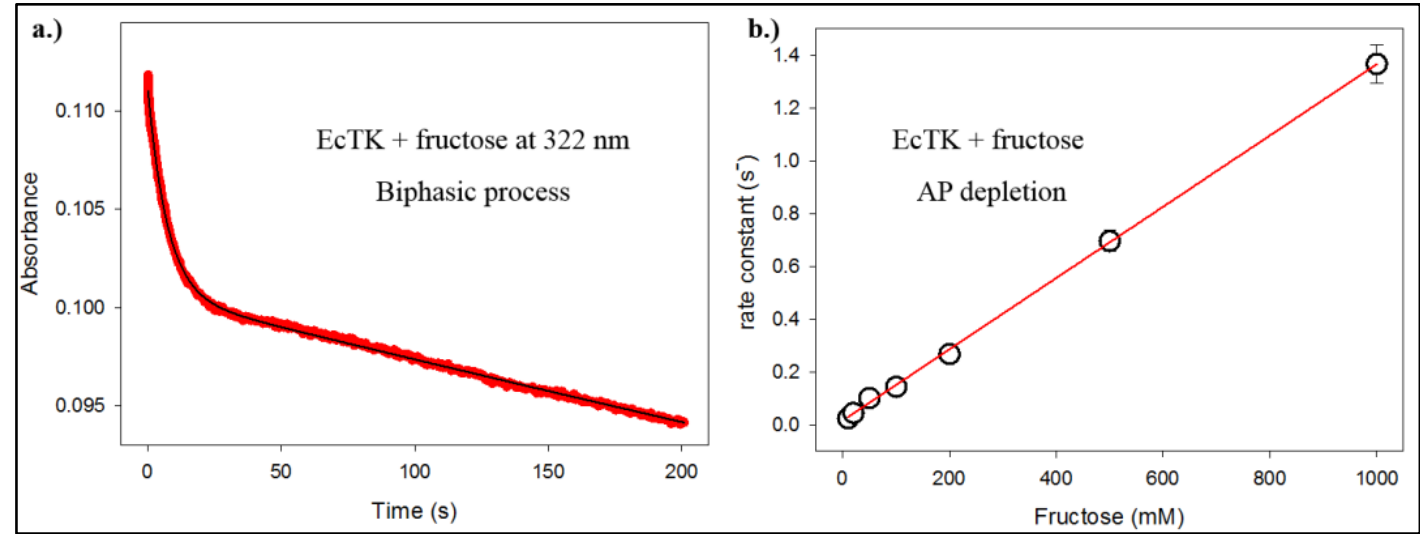

Fig 65: Single-mixing stopped-flow analysis of E.coli TK with fructose. $E c$ TK (4 mg/ml) was first supplemented with $300 \mu \mathrm{M}$ ThDP and incubated for $20 \mathrm{~min}$ to reconstitute the holo-enzyme, then mixed with increasing concentrations of fructose in $5 \mathrm{mM} \mathrm{CaCl}_{2}, 50 \mathrm{mM}$ glycylglycine (pH 7.6) and measured by a single-mixing stopped-flow at $322 \mathrm{~nm}$ at $4{ }^{\circ} \mathrm{C}$. a.) Representative stopped-flow reaction progress curve with $100 \mathrm{mM}$ fructose. A biphasic process is observed compared to human TK. b.) Plotting of the reaction velocity of AP depletion ( $322 \mathrm{~nm}$ ) against utilised fructose concentration. Please note that at same substrate concentration, the rate constants of E.coli TK is 40 times faster than that of the human TK. 

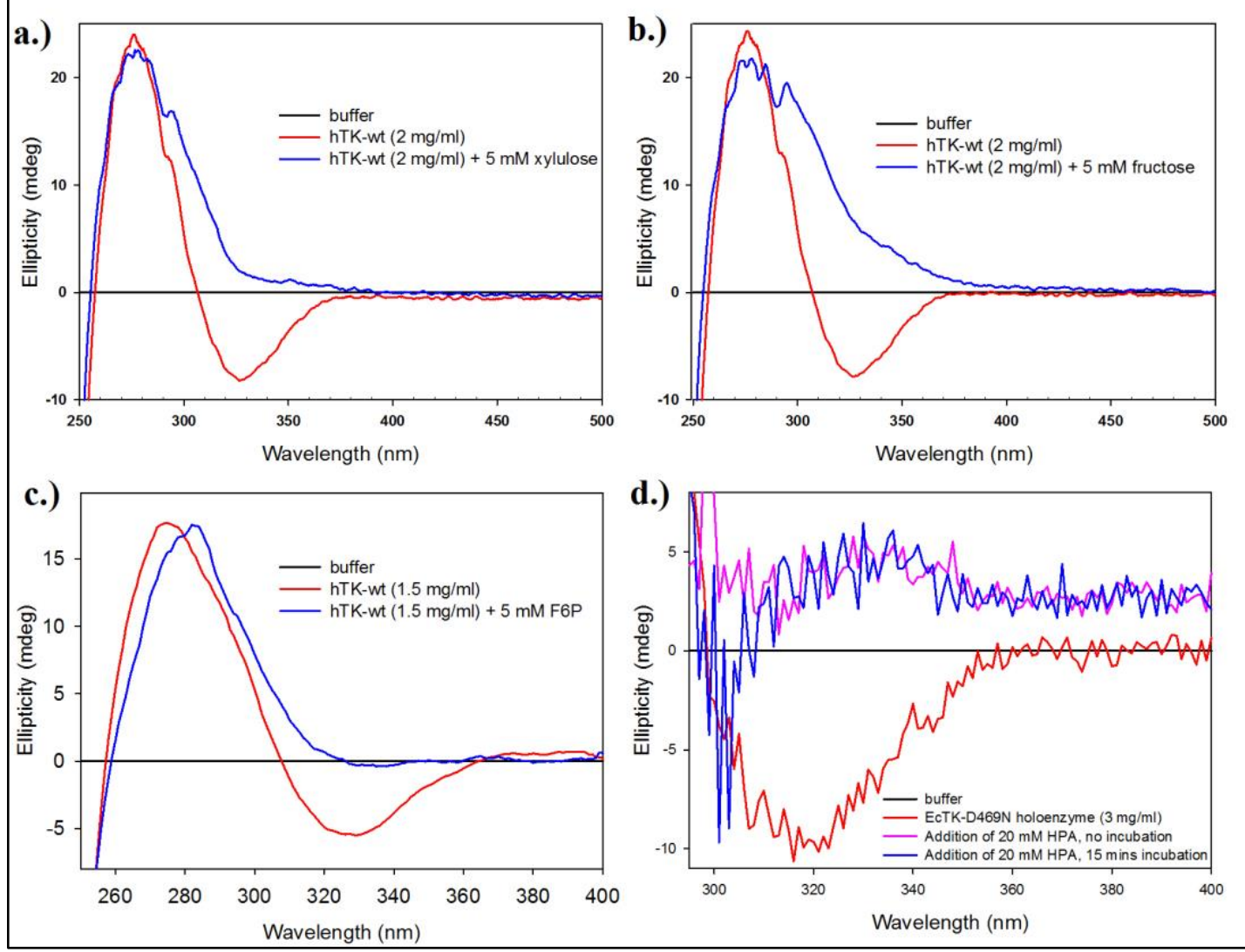

Fig 66: Circular Dichroism (CD) spectra of substrate binding to human and E.coli TK. Nonphosphorylated substrates xylulose and fructose, native substrate F6P and artificial substrate HPA were added to human and E.coli transketolase and the binding behaviours were measured by CD. For experimental details, please refer to Table 2. a.) The binding of xylulose to human TK. b.) The binding of fructose to human TK. c.) The binding of fructose 6-phosphate (F6P) to human TK. d.) The binding of hydroxypyruvate (HPA) to EcTK-D469N. Please note that the absorbance at $300 \mathrm{~nm}$ drops after 20 min incubation, indicating instability of the DHEThDP intermediate. 


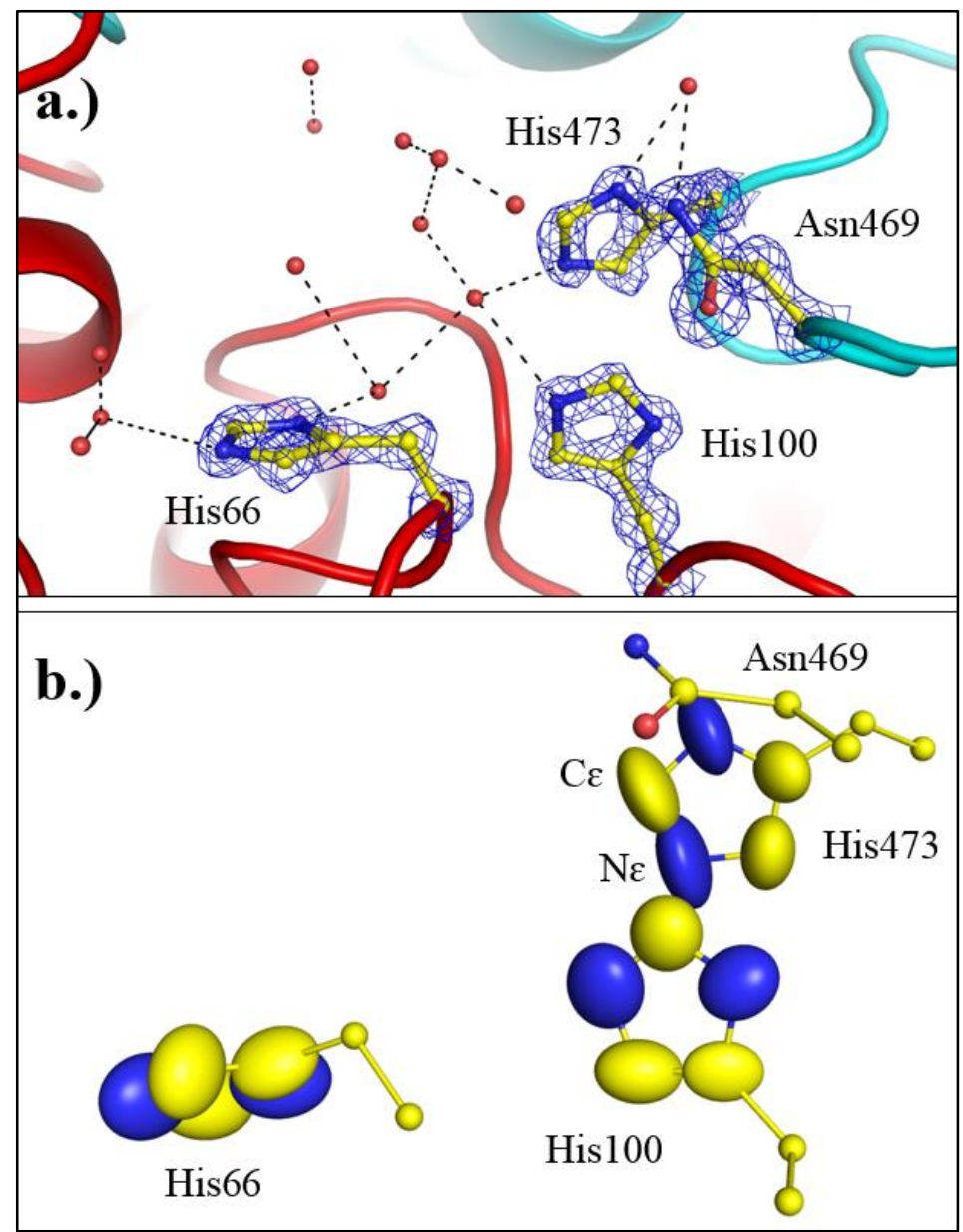

Fig 67: "Flipping histidine" observed in the active site of $E c$ TK D469N apo-enzyme. a.) Active site architecture of $E c$ TK D469N-apo at a resolution of 1.20 A. Amino acid residues His66, His100, His473 and Asn 469 are shown in ball-stick representation surrounded by a $2 \mathrm{mFo}-\mathrm{DFc}$ map (contoured at $2 \sigma$ level, blue). The electron density for His473 is more diffuse while others (His66 and His100) are welldefined. Several water molecules are observed at the position where cofactor ThDP should bind. Water molecules are shown as red spheres and hydrogen bonding interactions are shown as black dashed lines. b.) Anisotropic motion of His66, His100 and His473 in EcTK D469N apo-enzyme. The atomic displacement parameters (ADPs) of His473 indicate that $\mathrm{N} \varepsilon$ and $\mathrm{C} \varepsilon$ would thermally move along their bond axis, which is not possible because of rigid bond rule. This suggests that His473 exists in two conformation though ring flipping. 


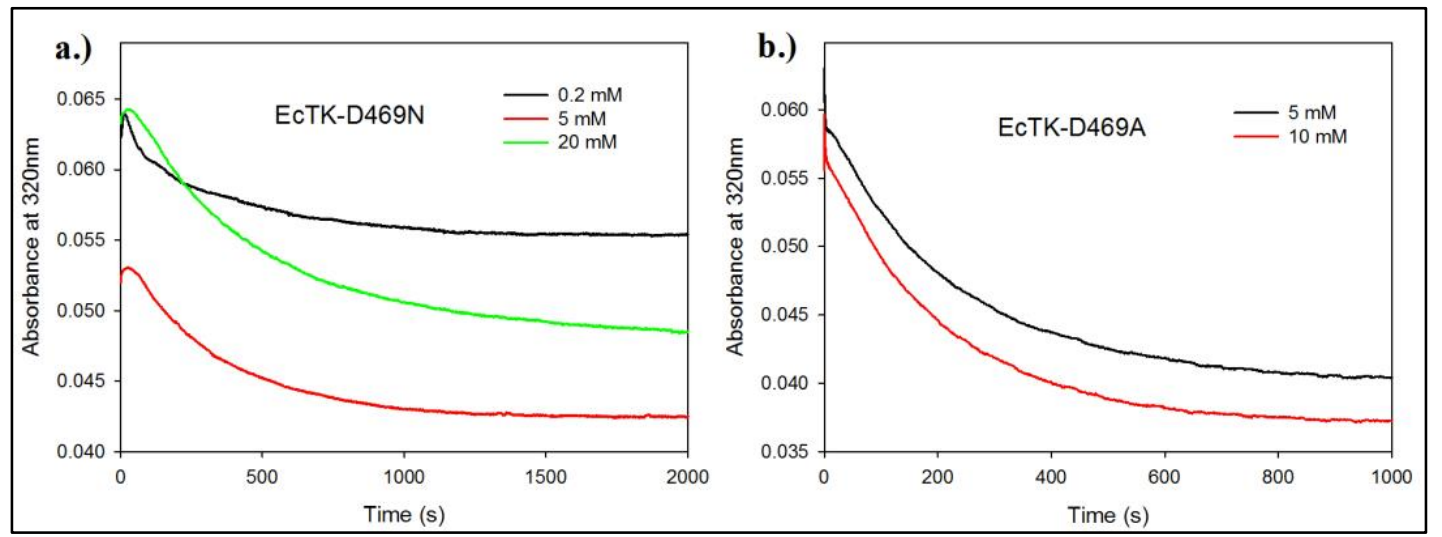

Fig 68: Single-mixing stopped-flow analysis of $E c$ TK-D469N and $E c$ TK-D469A with physiological substrate fructose 6-phosphate (F6P). EcTK $(4 \mathrm{mg} / \mathrm{ml})$ was first supplemented with $300 \mu \mathrm{M}$ ThDP and incubated for $20 \mathrm{~min}$ to reconstitute the holo-enzyme, then mixed with increasing concentrations of F6P in $5 \mathrm{mM} \mathrm{CaCl}_{2}, 50 \mathrm{mM}$ glycylglycine ( $\mathrm{pH} \mathrm{7.6)}$ and measured by a single-mixing stopped-flow at $320 \mathrm{~nm}$ at $25{ }^{\circ} \mathrm{C}$. a.) Representative stopped-flow reaction progress curve of EcTK-D469N. a.) Representative stopped-flow reaction progress curve of EcTK-D469A.
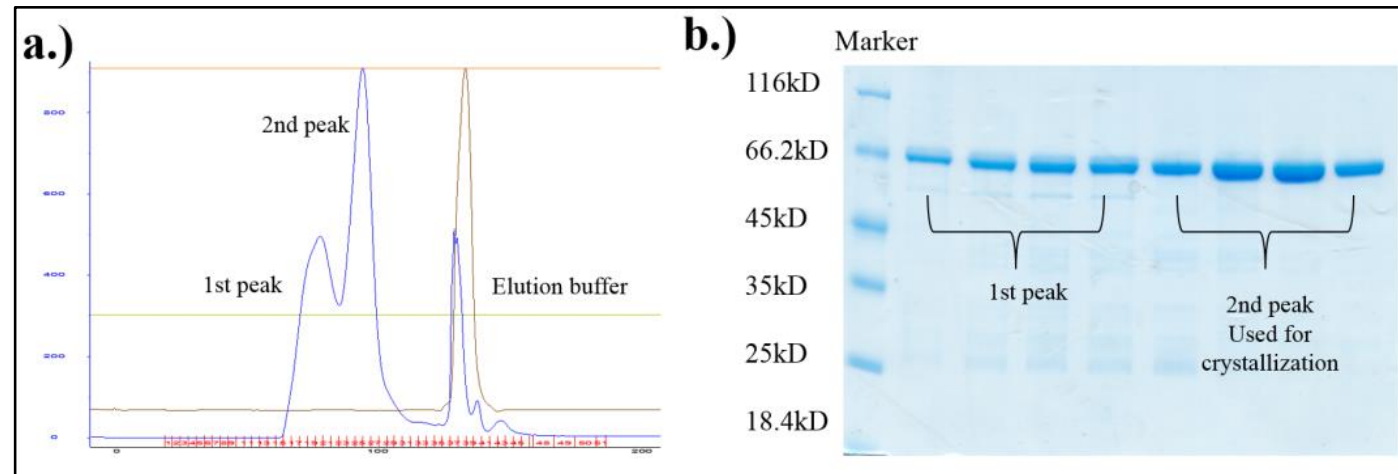

Fig 69: Purification of $\boldsymbol{h}$ TK for crystallization. In order to obtain $h \mathrm{TK}$ with high purity for crystallization purposes, a Superdex 200 (S200) column was used to separate the protein samples after Ni-NTA purification. a.) Typical chromatogram of the purification step using the S200 column. b.) SDS-PAGE analysis of the fractions eluted from S200 column. Please note that the protein samples from the second peak is more pure than the first one therefore was used in the crystallization experiments. 
Table 10: Analysis of the hydrogen bond distance at the same LBHB position in other transketolase structures. Transketolase structures with a resolution higher than $2 \AA$ are analysed.

\begin{tabular}{|c|c|c|c|c|}
\hline Organism & Enzyme stage & Distance $(\AA)$ & PDB code & Resolution (̊̊) \\
\hline \multirow[t]{5}{*}{ H. sapiens } & Ground state & 2.48 & $3 \mathrm{MOS}$ & 1.75 \\
\hline & F6P & 2.55 & $4 \mathrm{KXU}$ & 0.98 \\
\hline & $\mathrm{X} 5 \mathrm{P}$ & 2.56 & $4 \mathrm{KXV}$ & 0.97 \\
\hline & $\mathrm{X} 5 \mathrm{P}$ & 2.56 & $4 \mathrm{KXW}$ & 0.97 \\
\hline & S7P & 2.49 & $4 \mathrm{KXX}$ & 1.03 \\
\hline \multirow[t]{4}{*}{ E. coli } & Ground state & 2.51 & 1QGD & 1.90 \\
\hline & $\mathrm{X} 5 \mathrm{P}$ & 2.58 & $2 \mathrm{R} 8 \mathrm{O}$ & 1.47 \\
\hline & F6P & 2.54 & 2R8P & 1.65 \\
\hline & R5P & 2.57 & $2 \mathrm{R} 5 \mathrm{~N}$ & 1.60 \\
\hline \multirow[t]{2}{*}{ S. cerevisiae } & Ground state & 2.63 & $1 \mathrm{TRK}$ & 2.00 \\
\hline & HPA & 2.50 & $1 \mathrm{GPU}$ & 1.86 \\
\hline \multirow[t]{3}{*}{ P. Stipitis } & Ground state & 2.52 & $5 \mathrm{HYV}$ & 1.03 \\
\hline & Apo form & 2.51 & $5 \mathrm{I} 4 \mathrm{I}$ & 1.06 \\
\hline & R356L with F6P & 2.45 & $5 \mathrm{I} 51$ & 1.54 \\
\hline F. tularensis & Apo form & 2.52 & $3 \mathrm{KOM}$ & 1.60 \\
\hline \multirow[t]{2}{*}{ C. jejuni } & Ground state & 2.52 & $3 \mathrm{M} 34$ & 1.65 \\
\hline & R5P & 2.46 & 3M61 & 1.59 \\
\hline P. aeruginosa & Apo form & 2.45 & 4XEU & 1.95 \\
\hline
\end{tabular}




\section{References}

Adams, P.D., Afonine, P.V., Bunkoczi, G., Chen, V.B., Davis, I.W., Echols, N., Headd, J.J., Hung, L.W., Kapral, G.J., Grosse-Kunstleve, R.W., et al. (2010). PHENIX: a comprehensive Python-based system for macromolecular structure solution. Acta Crystallogr. Allen, F.H., Kennard, O., Watson, D.G., Brammer, L., Orpen, A.G., and Taylor, R. (1987). Tables of bond lengths determined by X-ray and neutron diffraction. Part 1. Bond lengths in organic compounds. J. Chem. Soc. Perkin Trans. 2 S1-S19.

Amara, P., Fdez Galván, I., Fontecilla-Camps, J.C., and Field, M.J. (2008). The enamine intermediate may not be universal to thiamine catalysis. Angew. Chem. Int. Ed Engl. 47, 628.

Amyes, T.L., and Richard, J.P. (2007). Enzymatic catalysis of proton transfer at carbon: activation of triosephosphate isomerase by phosphite dianion. Biochemistry (Mosc.) 46, 58415854 .

Amyes, T.L., Richard, J.P., and Tait, J.J. (2005). Activation of orotidine 5'-monophosphate decarboxylase by phosphite dianion: the whole substrate is the sum of two parts. J. Am. Chem. Soc. $127,15708-15709$.

Asztalos (2008). Untersuchungen zu molekularen, strukturellen und biokatalytischen Aspekten des Vitamin B1-abhängigen Enzyms Transketolase A aus Escherichia coli.

Asztalos, P., Parthier, C., Golbik, R., Kleinschmidt, M., Hübner, G., Weiss, M.S., Friedemann, R., Wille, G., and Tittmann, K. (2007). Strain and near attack conformers in enzymic thiamin catalysis: X-ray crystallographic snapshots of bacterial transketolase in covalent complex with donor ketoses xylulose 5-phosphate and fructose 6-phosphate, and in noncovalent complex with acceptor aldose ribose 5-phosphate. Biochemistry (Mosc.) 46, 12037-12052.

Aucamp, J.P., Martinez-Torres, R.J., Hibbert, E.G., and Dalby, P.A. (2008). A microplate-based evaluation of complex denaturation pathways: Structural stability of Escherichia coli transketolase. Biotechnol. Bioeng. 99, 1303-1310.

Barclay, T., Ginic-Markovic, M., Johnston, M.R., Cooper, P., and Petrovsky, N. (2012). Observation of the keto tautomer of D-fructose in D2O using 1H NMR spectroscopy. Carbohydr. Res. 347, 136-141.

Belenky, I., Steinmetz, A., Vyazmensky, M., Barak, Z., Tittmann, K., and Chipman, D.M. (2012). Many of the functional differences between acetohydroxyacid synthase (AHAS) 
isozyme I and other AHASs are a result of the rapid formation and breakdown of the covalent acetolactate-thiamin diphosphate adduct in AHAS I. FEBS J. 279, 1967-1979.

Berkessel, A., Elfert, S., Etzenbach-Effers, K., and Teles, J.H. (2010). Aldehyde Umpolung by N-Heterocyclic Carbenes: NMR Characterization of the Breslow Intermediate in its Keto Form, and a Spiro-Dioxolane as the Resting State of the Catalytic System. Angew. Chem. Int. Ed. 49, 7120-7124.

Berthold, C.L., Toyota, C.G., Moussatche, P., Wood, M.D., Leeper, F., Richards, N.G.J., and Lindqvist, Y. (2007). Crystallographic snapshots of oxalyl-CoA decarboxylase give insights into catalysis by nonoxidative ThDP-dependent decarboxylases. Struct. Lond. Engl. 1993 15, 853-861.

Bettendorff, L. (1994). The compartmentation of phosphorylated thiamine derivatives in cultured neuroblastoma cells. Biochim. Biophys. Acta 1222, 7-14.

Bettendorff, L., and Wins, P. (2009). Thiamin diphosphate in biological chemistry: new aspects of thiamin metabolism, especially triphosphate derivatives acting other than as cofactors. FEBS J. 276, 2917-2925.

Bettendorff, L., Wirtzfeld, B., Makarchikov, A.F., Mazzucchelli, G., Frédérich, M., Gigliobianco, T., Gangolf, M., De Pauw, E., Angenot, L., and Wins, P. (2007). Discovery of a natural thiamine adenine nucleotide. Nat. Chem. Biol. 3, 211-212.

Bornscheuer, U.T., Huisman, G.W., Kazlauskas, R.J., Lutz, S., Moore, J.C., and Robins, K. (2012). Engineering the third wave of biocatalysis. Nature 485, 185-194.

Bradford, M.M. (1976). A rapid and sensitive method for the quantitation of microgram quantities of protein utilizing the principle of protein-dye binding. Anal. Biochem. 72, 248254.

Breslow, R. (1957). RAPID DEUTERIUM EXCHANGE IN THIAZOLIUM SALTS1. J. Am. Chem. Soc. 79, 1762-1763.

Bruning, M., Berheide, M., Meyer, D., Golbik, R., Bartunik, H., Liese, A., and Tittmann, K. (2009). Structural and kinetic studies on native intermediates and an intermediate analogue in benzoylformate decarboxylase reveal a least motion mechanism with an unprecedented short-lived predecarboxylation intermediate. Biochemistry (Mosc.) 48, 3258-3268.

Bubb, W.A. (2003). NMR spectroscopy in the study of carbohydrates: Characterizing the structural complexity. Concepts Magn. Reson. Part A 19A, 1-19.

Bunik, V.I. (2013). Thiamin-dependent enzymes: new perspectives from the interface between chemistry and biology. FEBS J. 280, 6373. 
Bunik, V.I., Tylicki, A., and Lukashev, N.V. (2013). Thiamin diphosphate-dependent enzymes: from enzymology to metabolic regulation, drug design and disease models. FEBS J. $280,6412-6442$.

Burgi, H.B., Dunitz, J.D., and Shefter, E. (1973). Geometrical reaction coordinates. II. Nucleophilic addition to a carbonyl group. J. Am. Chem. Soc. 95, 5065-5067.

Burgi, H.B., Dunitz, J.D., Lehn, J.M., and Wipff, G. (1974). Stereochemistry of reaction paths at carbonyl centres. Tetrahedron 30, 1563-1572.

Candy, J.M., Koga, J., Nixon, P.F., and Duggleby, R.G. (1996). The role of residues glutamate-50 and phenylalanine-496 in Zymomonas mobilis pyruvate decarboxylase. Biochem. J. 315, 745-751.

Cassidy, C.S., Lin, J., and Frey, P.A. (1997). A new concept for the mechanism of action of chymotrypsin: the role of the low-barrier hydrogen bond. Biochemistry (Mosc.) 36, 45764584.

Cázares, A., Galman, J.L., Crago, L.G., Smith, M.E.B., Strafford, J., Ríos-Solís, L., Lye, G.J., Dalby, P.A., and Hailes, H.C. (2010). Non-alpha-hydroxylated aldehydes with evolved transketolase enzymes. Org. Biomol. Chem. 8, 1301-1309.

Chabrière, E., Vernède, X., Guigliarelli, B., Charon, M.H., Hatchikian, E.C., and Fontecilla-Camps, J.C. (2001). Crystal structure of the free radical intermediate of pyruvate:ferredoxin oxidoreductase. Science 294, 2559-2563.

Chipman, D.M., Duggleby, R.G., and Tittmann, K. (2005). Mechanisms of acetohydroxyacid synthases. Curr. Opin. Chem. Biol. 9, 475-481.

Ciszak, E.M., Korotchkina, L.G., Dominiak, P.M., Sidhu, S., and Patel, M.S. (2003). Structural basis for flip-flop action of thiamin pyrophosphate-dependent enzymes revealed by human pyruvate dehydrogenase. J. Biol. Chem. 278, 21240-21246.

Cleland, W.W. (2000). Low-barrier hydrogen bonds and enzymatic catalysis. Arch. Biochem. Biophys. 382, 1-5.

Cleland, W.W., and Kreevoy, M.M. (1994). Low-barrier hydrogen bonds and enzymic catalysis. Science $264,1887-1890$.

Cleland, W.W., Frey, P.A., and Gerlt, J.A. (1998). The Low Barrier Hydrogen Bond in Enzymatic Catalysis. J. Biol. Chem. 273, 25529-25532.

Collaborative Computational Project, Number 4 (1994). The CCP4 suite: programs for protein crystallography. Acta Crystallogr. D Biol. Crystallogr. 50, 760-763. 
Coy, J.F., Dressler, D., Wilde, J., and Schubert, P. (2005). Mutations in the transketolaselike gene TKTL1: clinical implications for neurodegenerative diseases, diabetes and cancer. Clin. Lab. 51, 257-273.

Crooks, J.E. (1983). The temperature-jump technique for the study of fast reactions in solution. J. Phys. [E] 16, 1142.

Crosby, J., and Lienhard, G.E. (1970). Mechanisms of thiamine-catalyzed reactions. Kinetic analysis of the decarboxylation of pyruvate by 3,4-dimethylthiazolium ion in water and ethanol. J. Am. Chem. Soc. 92, 5707-5716.

Davis, I.W., Leaver-Fay, A., Chen, V.B., Block, J.N., Kapral, G.J., Wang, X., Murray, L.W., Arendall, W.B., Snoeyink, J., Richardson, J.S., et al. (2007). MolProbity: all-atom contacts and structure validation for proteins and nucleic acids. Nucleic Acids Res. 35, W375383.

Du, J.-T., Li, Y.-M., Wei, W., Wu, G.-S., Zhao, Y.-F., Kanazawa, K., Nemoto, T., and Nakanishi, H. (2005). Low-barrier hydrogen bond between phosphate and the amide group in phosphopeptide. J. Am. Chem. Soc. 127, 16350-16351.

Du, Q., Wang, H., and Xie, J. (2011). Thiamin (Vitamin B1) Biosynthesis and Regulation: A Rich Source of Antimicrobial Drug Targets? Int. J. Biol. Sci. 7, 41-52.

Eisenmesser, E.Z., Millet, O., Labeikovsky, W., Korzhnev, D.M., Wolf-Watz, M., Bosco, D.A., Skalicky, J.J., Kay, L.E., and Kern, D. (2005). Intrinsic dynamics of an enzyme underlies catalysis. Nature 438, 117-121.

Eisinger, J., Arroyo, P., Braquet, M., Arroyo, H., and Ayavou, T. (1994). [Erythrocyte transketolases and Alzheimer disease]. Rev. Médecine Interne Fondée Par Société Natl. Francaise Médecine Interne 15, 387-389.

Elias, M., Wellner, A., Goldin-Azulay, K., Chabriere, E., Vorholt, J.A., Erb, T.J., and Tawfik, D.S. (2012). The molecular basis of phosphate discrimination in arsenate-rich environments. Nature 491, 134-137.

Emsley, P., Lohkamp, B., Scott, W.G., and Cowtan, K. (2010). Features and development of Coot. Acta Crystallogr. D Biol. Crystallogr. 66, 486-501.

Fiedler, E., Thorell, S., Sandalova, T., Golbik, R., König, S., and Schneider, G. (2002). Snapshot of a key intermediate in enzymatic thiamin catalysis: crystal structure of the alphacarbanion of (alpha,beta-dihydroxyethyl)-thiamin diphosphate in the active site of transketolase from Saccharomyces cerevisiae. Proc. Natl. Acad. Sci. U. S. A. 99, 591-595. 
Frank, R. a. W., Leeper, F.J., and Luisi, B.F. (2007a). Structure, mechanism and catalytic duality of thiamine-dependent enzymes. Cell. Mol. Life Sci. CMLS 64, 892-905.

Frank, R.A.W., Titman, C.M., Pratap, J.V., Luisi, B.F., and Perham, R.N. (2004). A molecular switch and proton wire synchronize the active sites in thiamine enzymes. Science 306, 872-876.

Frank, R.A.W., Price, A.J., Northrop, F.D., Perham, R.N., and Luisi, B.F. (2007b). Crystal Structure of the E1 Component of the Escherichia coli 2-Oxoglutarate Dehydrogenase Multienzyme Complex. J. Mol. Biol. 368, 639-651.

French, C., and Ward, J.M. (1995). Improved production and stability of E.coli recombinants expressing transketolase for large scale biotransformation. Biotechnol. Lett. 17, $247-252$.

Frey, P.A., Whitt, S.A., and Tobin, J.B. (1994). A low-barrier hydrogen bond in the catalytic triad of serine proteases. Science 264, 1927-1930.

Friedemann, R., and Breitkopf, C. (1996). Theoretical studies on the decarboxylation reaction in thiamin catalysis. Int. J. Quantum Chem. 57, 943-948.

Fry, K., Ingraham, L.L., and Westheimer, F.H. (1957). The Thiamin-Pyruvate Reaction. J. Am. Chem. Soc. 79, 5225-5227.

Fuhrmann, C.N., Daugherty, M.D., and Agard, D.A. (2006). Subangstrom crystallography reveals that short ionic hydrogen bonds, and not a His-Asp low-barrier hydrogen bond, stabilize the transition state in serine protease catalysis. J. Am. Chem. Soc. 128, 9086-9102.

Fullam, E., Pojer, F., Bergfors, T., Jones, T.A., and Cole, S.T. (2012). Structure and function of the transketolase from Mycobacterium tuberculosis and comparison with the human enzyme. Open Biol. 2, 110026.

Gedi, V., and Yoon, M.-Y. (2012). Bacterial acetohydroxyacid synthase and its inhibitors--a summary of their structure, biological activity and current status. FEBS J. 279, 946-963.

Gerhardt, S., Echt, S., Busch, M., Freigang, J., Auerbach, G., Bader, G., Martin, W.F., Bacher, A., Huber, R., and Fischer, M. (2003). Structure and properties of an engineered transketolase from maize. Plant Physiol. 132, 1941-1949.

Gerlt, J.A., and Gassman, P.G. (1993a). An explanation for rapid enzyme-catalyzed proton abstraction from carbon acids: importance of late transition states in concerted mechanisms. J. Am. Chem. Soc. 115, 11552-11568. 
Gerlt, J.A., and Gassman, P.G. (1993b). Understanding the rates of certain enzymecatalyzed reactions: proton abstraction from carbon acids, acyl-transfer reactions, and displacement reactions of phosphodiesters. Biochemistry (Mosc.) 32, 11943-11952.

Golbik, R., Meshalkina, L.E., Sandalova, T., Tittmann, K., Fiedler, E., Neef, H., König, S., Kluger, R., Kochetov, G.A., Schneider, G., et al. (2005). Effect of coenzyme modification on the structural and catalytic properties of wild-type transketolase and of the variant E418A from Saccharomyces cerevisiae. FEBS J. 272, 1326-1342.

Goldman, L.M., Amyes, T.L., Goryanova, B., Gerlt, J.A., and Richard, J.P. (2014). Enzyme architecture: deconstruction of the enzyme-activating phosphodianion interactions of orotidine 5'-monophosphate decarboxylase. J. Am. Chem. Soc. 136, 10156-10165.

Goselink, R.J.M., Harlaar, J.J., Vermeij, F.H., Biter, U.L.U., Mannaerts, G.H.H., and den Heijer, T. (2012). [Beriberi after bariatric surgery]. Ned. Tijdschr. Geneeskd. 155, A4500.

Gouet, P., Courcelle, E., Stuart, D.I., and Métoz, F. (1999). ESPript: analysis of multiple sequence alignments in PostScript. Bioinforma. Oxf. Engl. 15, 305-308.

Grabski, A., Mehler, M., and Drott, D. (2005). The Overnight Express Autoinduction System: High-density cell growth and protein expression while you sleep. Nat. Methods 2, 233-235.

Graham, J.D., Buytendyk, A.M., Wang, D., Bowen, K.H., and Collins, K.D. (2014). Strong, Low-Barrier Hydrogen Bonds May Be Available to Enzymes. Biochemistry (Mosc.) $53,344-349$.

Guérard, C., Alphand, V., Archelas, A., Demuynck, C., Hecquet, L., Furstoss, R., and Bolte, J. (1999). Transketolase-Mediated Synthesis of 4-Deoxy-D-fructose 6-Phosphate by Epoxide Hydrolase-Catalysed Resolution of 1,1-Diethoxy-3,4-epoxybutane. Eur. J. Org. Chem. 1999, 3399-3402.

Gutfreund, H. (1981). Kinetics of Fast Enzyme Reactions: Theory and Practice. Biochem. Soc. Trans. 9, 177-178.

Hailes, H.C., Rother, D., Müller, M., Westphal, R., Ward, J.M., Pleiss, J., Vogel, C., and Pohl, M. (2013). Engineering stereoselectivity of ThDP-dependent enzymes. FEBS J. 280, 6374-6394.

Hammes, H.-P., Du, X., Edelstein, D., Taguchi, T., Matsumura, T., Ju, Q., Lin, J., Bierhaus, A., Nawroth, P., Hannak, D., et al. (2003). Benfotiamine blocks three major 
pathways of hyperglycemic damage and prevents experimental diabetic retinopathy. Nat. Med. 9, 294-299.

Hansen, D.E., and Raines, R.T. (1990). Binding energy and enzymatic catalysis. J. Chem. Educ. 67, 483.

Hecquet, L., Bolte, J., and Demuynck, C. (1996). Enzymatic synthesis of "natural-labeled" 6-deoxy-L-sorbose precursor of an important food flavor. Tetrahedron 52, 8223-8232.

Hibbert, E.G., Senussi, T., Costelloe, S.J., Lei, W., Smith, M.E.B., Ward, J.M., Hailes, H.C., and Dalby, P.A. (2007). Directed evolution of transketolase activity on nonphosphorylated substrates. J. Biotechnol. 131, 425-432.

Hobbs, G.R., Lilly, M.D., Turner, N.J., Ward, J.M., Willets, A.J., and Woodley, J.M. (1993). Enzyme-catalysed carbon?carbon bond formation: use of transketolase from Escherichia coli. ResearchGate 1 .

Hübner, G., Tittmann, K., Killenberg-Jabs, M., Schäffner, J., Spinka, M., Neef, H., Kern, D., Kern, G., Schneider, G., Wikner, C., et al. (1998). Activation of thiamin diphosphate in enzymes1. Biochim. Biophys. Acta BBA - Protein Struct. Mol. Enzymol. 1385, 221-228.

Inoue, H., Nojima, H., and Okayama, H. (1990). High efficiency transformation of Escherichia coli with plasmids. Gene 96, 23-28.

Ishikita, H., and Saito, K. (2014). Proton transfer reactions and hydrogen-bond networks in protein environments. J. R. Soc. Interface R. Soc. 11, 20130518.

Jang, D.S., Choi, G., Cha, H.J., Shin, S., Hong, B.H., Lee, H.J., Lee, H.C., and Choi, K.Y. (2015). Contribution of a low-barrier hydrogen bond to catalysis is not significant in ketosteroid isomerase. Mol. Cells 38, 409-415.

Jordan, F. (2003). Current mechanistic understanding of thiamin diphosphate-dependent enzymatic reactions. Nat. Prod. Rep. 20, 184-201.

Jordan, F. (2004). Biochemistry. How active sites communicate in thiamine enzymes. Science $306,818-820$.

Jordan, F., Zhang, Z., and Sergienko, E. (2002). Spectroscopic evidence for participation of the 1',4'-imino tautomer of thiamin diphosphate in catalysis by yeast pyruvate decarboxylase. Bioorganic Chem. 30, 188-198.

Kabsch, W. (2010). XDS. Acta Crystallogr. D Biol. Crystallogr. 66, 125-132. 
Kaplun, A., Binshtein, E., Vyazmensky, M., Steinmetz, A., Barak, Z. 'ev, Chipman, D.M., Tittmann, K., and Shaanan, B. (2008). Glyoxylate carboligase lacks the canonical active site glutamate of thiamine-dependent enzymes. Nat. Chem. Biol. 4, 113-118.

Keller, M.A., Turchyn, A.V., and Ralser, M. (2014). Non-enzymatic glycolysis and pentose phosphate pathway-like reactions in a plausible Archean ocean. Mol. Syst. Biol. 10, 725.

Kern, D., Kern, G., Neef, H., Tittmann, K., Killenberg-Jabs, M., Wikner, C., Schneider, G., and Hübner, G. (1997). How thiamine diphosphate is activated in enzymes. Science 275, $67-70$.

Kholodar, S.A., Allen, C.L., Gulick, A.M., and Murkin, A.S. (2015). The role of phosphate in a multistep enzymatic reaction: reactions of the substrate and intermediate in pieces. J. Am. Chem. Soc. 137, 2748-2756.

Klinman, J.P. (2015). Low Barrier Hydrogen Bonds: Getting Close, but Not Sharing... ACS Cent. Sci. $1,115-116$.

Kluger, R. (1990). Ionic intermediates in enzyme-catalyzed carbon-carbon bond formation: patterns, prototypes, probes, and proposals. Chem. Rev. 90, 1151-1169.

Kluger, R., and Tittmann, K. (2008). Thiamin diphosphate catalysis: enzymic and nonenzymic covalent intermediates. Chem. Rev. 108, 1797-1833.

Kochetov, G.A. (1982). Structure of the active center of transketolase. Ann. N. Y. Acad. Sci. 378, 306-311.

Kruger, N.J., and von Schaewen, A. (2003). The oxidative pentose phosphate pathway: structure and organisation. Curr. Opin. Plant Biol. 6, 236-246.

Kuhn, P., Knapp, M., Soltis, S.M., Ganshaw, G., Thoene, M., and Bott, R. (1998). The $0.78 \AA$ A structure of a serine protease: Bacillus lentus subtilisin. Biochemistry (Mosc.) 37, 13446-13452.

Kuo, D.J., and Jordan, F. (1983). Direct spectroscopic observation of a brewer's yeast pyruvate decarboxylase-bound enamine intermediate produced from a suicide substrate. Evidence for nonconcerted decarboxylation. J. Biol. Chem. 258, 13415-13417.

Kutter, S., Weiss, M.S., Wille, G., Golbik, R., Spinka, M., and König, S. (2009). Covalently bound substrate at the regulatory site of yeast pyruvate decarboxylases triggers allosteric enzyme activation. J. Biol. Chem. 284, 12136-12144.

Laemmli, U.K. (1970). Cleavage of structural proteins during the assembly of the head of bacteriophage T4. Nature 227, 680-685. 
Larkin, M.A., Blackshields, G., Brown, N.P., Chenna, R., McGettigan, P.A., McWilliam, H., Valentin, F., Wallace, I.M., Wilm, A., Lopez, R., et al. (2007). Clustal W and Clustal X version 2.0. Bioinforma. Oxf. Engl. 23, 2947-2948.

LaRossa, R.A., and Schloss, J.V. (1984). The sulfonylurea herbicide sulfometuron methyl is an extremely potent and selective inhibitor of acetolactate synthase in Salmonella typhimurium. J. Biol. Chem. 259, 8753-8757.

Lehwess-Litzmann, A., Neumann, P., Parthier, C., Lüdtke, S., Golbik, R., Ficner, R., and Tittmann, K. (2011). Twisted Schiff base intermediates and substrate locale revise transaldolase mechanism. Nat. Chem. Biol. 7, 678-684.

Lichtenthaler, H.K. (1999). The 1-Deoxy-D-Xylulose-5-Phosphate Pathway of Isoprenoid Biosynthesis in Plants. Annu. Rev. Plant Physiol. Plant Mol. Biol. 50, 47-65.

Lichtenthaler, H.K. (2000). Non-mevalonate isoprenoid biosynthesis: enzymes, genes and inhibitors. Biochem. Soc. Trans. 28, 785-789.

Lindqvist, Y., Schneider, G., Ermler, U., and Sundström, M. (1992). Three-dimensional structure of transketolase, a thiamine diphosphate dependent enzyme, at $2.5 \AA$ resolution. EMBO J. 11, 2373-2379.

Littlechild, J., Turner, N., Hobbs, G., Lilly, M., Rawas, A., and Watson, H. (1995). Crystallization and preliminary X-ray crystallographic data with Escherichia coli transketolase. Acta Crystallogr. D Biol. Crystallogr. 51, 1074-1076.

Lobell, M., and Crout, D.H.G. (1996). Pyruvate Decarboxylase: A Molecular Modeling Study of Pyruvate Decarboxylation and Acyloin Formation. J. Am. Chem. Soc. 118, 18671873.

Lüdtke, S. (2012). Structural and Funtional Studies on VitaminB1-Dependent Human and Bacterial Transketolases.

Lüdtke, S., Neumann, P., Erixon, K.M., Leeper, F., Kluger, R., Ficner, R., and Tittmann, K. (2013). Sub-ångström-resolution crystallography reveals physical distortions that enhance reactivity of a covalent enzymatic intermediate. Nat. Chem. 5, 762-767.

Lukacik, P., Lobley, C.M.C., Bumann, M., Arena de Souza, V., Owens, R.J., O’Toole, P.W., and Walsh, M.A. (2015). High-resolution structures of Lactobacillus salivarius transketolase in the presence and absence of thiamine pyrophosphate. Acta Crystallogr. Sect. F Struct. Biol. Commun. 71, 1327-1334.

Machius, M., Wynn, R.M., Chuang, J.L., Li, J., Kluger, R., Yu, D., Tomchick, D.R., Brautigam, C.A., and Chuang, D.T. (2006). A versatile conformational switch regulates 
reactivity in human branched-chain alpha-ketoacid dehydrogenase. Struct. Lond. Engl. 1993 14, 287-298.

Malabanan, M.M., Amyes, T.L., and Richard, J.P. (2011). Mechanism for activation of triosephosphate isomerase by phosphite dianion: the role of a ligand-driven conformational change. J. Am. Chem. Soc. 133, 16428-16431.

Matsue, Y., Mizuno, H., Tomita, T., Asami, T., Nishiyama, M., and Kuzuyama, T. (2010). The herbicide ketoclomazone inhibits 1-deoxy-D-xylulose 5-phosphate synthase in the 2-Cmethyl-D-erythritol 4-phosphate pathway and shows antibacterial activity against Haemophilus influenzae. J. Antibiot. (Tokyo) 63, 583-588.

Meshalkina, L., Nilsson, U., Wikner, C., Kostikowa, T., and Schneider, G. (1997). Examination of the Thiamin Diphosphate Binding Site in Yeast Transketolase by SiteDirected Mutagenesis. Eur. J. Biochem. 244, 646-652.

Meyer, D., Neumann, P., Parthier, C., Friedemann, R., Nemeria, N., Jordan, F., and Tittmann, K. (2010). Double duty for a conserved glutamate in pyruvate decarboxylase: evidence of the participation in stereoelectronically controlled decarboxylation and in protonation of the nascent carbanion/enamine intermediate. Biochemistry (Mosc.) 49, $8197-$ 8212.

Meyer, D., Neumann, P., Koers, E., Sjuts, H., Lüdtke, S., Sheldrick, G.M., Ficner, R., and Tittmann, K. (2012). Unexpected tautomeric equilibria of the carbanion-enamine intermediate in pyruvate oxidase highlight unrecognized chemical versatility of thiamin. Proc. Natl. Acad. Sci. U. S. A. 109, 10867-10872.

Meyer, D., Neumann, P., Ficner, R., and Tittmann, K. (2013). Observation of a stable carbene at the active site of a thiamin enzyme. Nat. Chem. Biol. 9, 488-490.

Mitschke, L., Parthier, C., Schröder-Tittmann, K., Coy, J., Lüdtke, S., and Tittmann, K. (2010). The crystal structure of human transketolase and new insights into its mode of action. J. Biol. Chem. 285, 31559-31570.

Mueller, C., Schwender, J., Zeidler, J., and Lichtenthaler, H.K. (2000). Properties and inhibition of the first two enzymes of the non-mevalonate pathway of isoprenoid biosynthesis. Biochem. Soc. Trans. 28, 792-793.

Muller, Y.A., Lindqvist, Y., Furey, W., Schulz, G.E., Jordan, F., and Schneider, G. (1993). A thiamin diphosphate binding fold revealed by comparison of the crystal structures of transketolase, pyruvate oxidase and pyruvate decarboxylase. Structure 1, 95-103. 
Nadal-Ferret, M., Gelabert, R., Moreno, M., and Lluch, J.M. (2014). Are There Really Low-Barrier Hydrogen Bonds in Proteins? The Case of Photoactive Yellow Protein. J. Am. Chem. Soc. 136, 3542-3552.

Nauton, L., Hélaine, V., Théry, V., and Hecquet, L. (2016). Insights into the Thiamine Diphosphate Enzyme Activation Mechanism: Computational Model for Transketolase Using a Quantum Mechanical/Molecular Mechanical Method. Biochemistry (Mosc.) 55, 2144-2152.

Nemeria, N., Baykal, A., Joseph, E., Zhang, S., Yan, Y., Furey, W., and Jordan, F. (2004). Tetrahedral intermediates in thiamin diphosphate-dependent decarboxylations exist as a 1',4'imino tautomeric form of the coenzyme, unlike the michaelis complex or the free coenzyme. Biochemistry (Mosc.) 43, 6565-6575.

Nemeria, N., Chakraborty, S., Baykal, A., Korotchkina, L.G., Patel, M.S., and Jordan, F. (2007). The 1',4'-iminopyrimidine tautomer of thiamin diphosphate is poised for catalysis in asymmetric active centers on enzymes. Proc. Natl. Acad. Sci. 104, 78-82.

Nemeria, N.S., Chakraborty, S., Balakrishnan, A., and Jordan, F. (2009). Reaction mechanisms of thiamin diphosphate enzymes: defining states of ionization and tautomerization of the cofactor at individual steps. FEBS J. 276, 2432-2446.

Nemeria, N.S., Arjunan, P., Chandrasekhar, K., Mossad, M., Tittmann, K., Furey, W., and Jordan, F. (2010). Communication between thiamin cofactors in the Escherichia coli pyruvate dehydrogenase complex E1 component active centers: evidence for a "direct pathway" between the 4'-aminopyrimidine N1' atoms. J. Biol. Chem. 285, 11197-11209.

Neumann, P., and Tittmann, K. (2014). Marvels of enzyme catalysis at true atomic resolution: distortions, bond elongations, hidden flips, protonation states and atom identities. Curr. Opin. Struct. Biol. 29, 122-133.

Nichols, D.A., Hargis, J.C., Sanishvili, R., Jaishankar, P., Defrees, K., Smith, E.W., Wang, K.K., Prati, F., Renslo, A.R., Woodcock, H.L., et al. (2015). Ligand-Induced Proton Transfer and Low-Barrier Hydrogen Bond Revealed by X-ray Crystallography. J. Am. Chem. Soc. $137,8086-8095$.

Nilsson, U., Hecquet, L., Gefflaut, T., Guerard, C., and Schneider, G. (1998). Asp477 is a determinant of the enantioselectivity in yeast transketolase. FEBS Lett. 424, 49-52.

Oltrogge, L.M., and Boxer, S.G. (2015). Short Hydrogen Bonds and Proton Delocalization in Green Fluorescent Protein (GFP). ACS Cent. Sci. 1, 148-156. 
Payongsri, P., Steadman, D., Hailes, H.C., and Dalby, P.A. (2015). Second generation engineering of transketolase for polar aromatic aldehyde substrates. Enzyme Microb. Technol. $71,45-52$.

Pierce, J., Serianni, A.S., and Barker, R. (1985). Anomerization of furanose sugars and sugar phosphates. J. Am. Chem. Soc. 107, 2448-2456.

Racker, E., Haba, G.D.L., and Leder, I.G. (1953). THIAMINE PYROPHOSPHATE, A COENZYME OF TRANSKETOLASE. J. Am. Chem. Soc. 75, 1010-1011.

Raines, C.A. (2003). The Calvin cycle revisited. Photosynth. Res. 75, 1-10.

Rebek, J. (1990). On the structure of histidine and its role in enzyme active sites. Struct. Chem. 1, 129-131.

Reyes, A.C., Zhai, X., Morgan, K.T., Reinhardt, C.J., Amyes, T.L., and Richard, J.P. (2015). The activating oxydianion binding domain for enzyme-catalyzed proton transfer, hydride transfer, and decarboxylation: specificity and enzyme architecture. J. Am. Chem. Soc. 137, 1372-1382.

Rindi, G. (1982). Metabolism of thiamin and its phosphoric esters in different regions of the nervous system: a new approach. Acta Vitaminol. Enzymol. 4, 59-68.

Rohdich, F., Kis, K., Bacher, A., and Eisenreich, W. (2001). The non-mevalonate pathway of isoprenoids: genes, enzymes and intermediates. Curr. Opin. Chem. Biol. 5, 535-540.

Sautner, V., Friedrich, M.M., Lehwess-Litzmann, A., and Tittmann, K. (2015). Converting Transaldolase into Aldolase through Swapping of the Multifunctional Acid-Base Catalyst: Common and Divergent Catalytic Principles in F6P Aldolase and Transaldolase. Biochemistry (Mosc.) 54, 4475-4486.

Sax, C.M., Salamon, C., Kays, W.T., Guo, J., Yu, F.X., Cuthbertson, R.A., and Piatigorsky, J. (1996). Transketolase is a major protein in the mouse cornea. J. Biol. Chem. $271,33568-33574$.

Schellenberger, A. (1998). Sixty years of thiamin diphosphate biochemistry. Biochim. Biophys. Acta 1385, 177-186.

Schenk, G., Duggleby, R.G., and Nixon, P.F. (1998). Properties and functions of the thiamin diphosphate dependent enzyme transketolase. Int. J. Biochem. Cell Biol. 30, 1297-1318.

Schneider, S. (2014). Functional characterization of transketolase-like proteins and related model systems with respect to thiamin diphosphate mediated chemistry. 
Schneider, G., and Lindqvist, Y. (1998). Crystallography and mutagenesis of transketolase: mechanistic implications for enzymatic thiamin catalysis. Biochim. Biophys. Acta BBA Protein Struct. Mol. Enzymol. 1385, 387-398.

Schneider, S., Lüdtke, S., Schröder-Tittmann, K., Wechsler, C., Meyer, D., and Tittmann, K. (2012). A $\delta 38$ deletion variant of human transketolase as a model of transketolase-like protein 1 exhibits no enzymatic activity. PloS One 7, e48321.

Schüttelkopf, A.W., and van Aalten, D.M.F. (2004). PRODRG: a tool for high-throughput crystallography of protein-ligand complexes. Acta Crystallogr. D Biol. Crystallogr. 60, 13551363.

Schütz, A., Golbik, R., König, S., Hübner, G., and Tittmann, K. (2005). Intermediates and transition states in thiamin diphosphate-dependent decarboxylases. A kinetic and NMR study on wild-type indolepyruvate decarboxylase and variants using indolepyruvate, benzoylformate, and pyruvate as substrates. Biochemistry (Mosc.) 44, 6164-6179.

Sergienko, E.A., Wang, J., Polovnikova, L., Hasson, M.S., McLeish, M.J., Kenyon, G.L., and Jordan, F. (2000). Spectroscopic detection of transient thiamin diphosphate-bound intermediates on benzoylformate decarboxylase. Biochemistry (Mosc.) 39, 13862-13869.

Serianni, A.S., Pierce, J., and Barker, R. (1979). Carbon-13-enriched carbohydrates: preparation of triose, tetrose, and pentose phosphates. Biochemistry (Mosc.) 18, 1192-1199.

Serianni, A.S., Pierce, J., Huang, S.G., and Barker, R. (1982). Anomerization of furanose sugars: Kinetics of ring-opening reactions by $1 \mathrm{H}$ and $13 \mathrm{C}$ saturation-transfer NMR spectroscopy. ResearchGate 104.

Shaanan, B., and Chipman, D.M. (2009). Reaction mechanisms of thiamin diphosphate enzymes: new insights into the role of a conserved glutamate residue. FEBS J. 276, 24472453.

Shaeri, J., Wright, I., Rathbone, E.B., Wohlgemuth, R., and Woodley, J.M. (2008). Characterization of enzymatic D-xylulose 5-phosphate synthesis. Biotechnol. Bioeng. 101, 761-767.

Smith, M.E.B., Hibbert, E.G., Jones, A.B., Dalby, P.A., and Hailes, H.C. (2008). Enhancing and Reversing the Stereoselectivity of Escherichia coli Transketolase via SinglePoint Mutations. Adv. Synth. Catal. 350, 2631-2638.

Soderberg, T. (2005). Biosynthesis of ribose-5-phosphate and erythrose-4-phosphate in archaea: a phylogenetic analysis of archaeal genomes. Archaea Vanc. BC 1, 347-352. 
Sprenger, G.A., Schörken, U., Sprenger, G., and Sahm, H. (1995). Transketolase A of Escherichia coli K12. Purification and properties of the enzyme from recombinant strains. Eur. J. Biochem. FEBS 230, 525-532.

Srere, P., Cooper, J.R., Tabachnick, M., and Racker, E. (1958). The oxidative pentose phosphate cycle. I. Preparation of substrates and enzymes. Arch. Biochem. Biophys. 74, 295305.

Stroh, C., Meyer, F., and Manger, T. (2014). Beriberi, a severe complication after metabolic surgery - review of the literature. Obes. Facts 7, 246-252.

Studier, F.W. (2005). Protein production by auto-induction in high-density shaking cultures. Protein Expr. Purif. 41, 207-234.

Suzuki, R., Katayama, T., Kim, B.-J., Wakagi, T., Shoun, H., Ashida, H., Yamamoto, K., and Fushinobu, S. (2010). Crystal structures of phosphoketolase: thiamine diphosphatedependent dehydration mechanism. J. Biol. Chem. 285, 34279-34287.

Takayama, S., McGarvey, G.J., and Wong, C.H. (1997). Microbial aldolases and transketolases: new biocatalytic approaches to simple and complex sugars. Annu. Rev. Microbiol. 51, 285-310.

Tamada, T., Kinoshita, T., Kurihara, K., Adachi, M., Ohhara, T., Imai, K., Kuroki, R., and Tada, T. (2009). Combined high-resolution neutron and X-ray analysis of inhibited elastase confirms the active-site oxyanion hole but rules against a low-barrier hydrogen bond. J. Am. Chem. Soc. 131, 11033-11040.

Tittmann, K. (2014). Sweet siblings with different faces: The mechanisms of FBP and F6P aldolase, transaldolase, transketolase and phosphoketolase revisited in light of recent structural data. Bioorganic Chem. 57, 263-280.

Tittmann, K., and Wille, G. (2009). X-ray crystallographic snapshots of reaction intermediates in pyruvate oxidase and transketolase illustrate common themes in thiamin catalysis. J. Mol. Catal. B Enzym. 61, 93-99.

Tittmann, K., Golbik, R., Hübner, G., and Ghisla, S. (2002). Phosphate mediates electron transfer in pyruvate oxidase from Lactobacillus plantarum. pp. 659-662.

Tittmann, K., Golbik, R., Uhlemann, K., Khailova, L., Schneider, G., Patel, M., Jordan, F., Chipman, D.M., Duggleby, R.G., and Hübner, G. (2003). NMR Analysis of Covalent Intermediates in Thiamin Diphosphate Enzymes. Biochemistry (Mosc.) 42, 7885-7891. 
Tsang, W.-Y., Amyes, T.L., and Richard, J.P. (2008). A substrate in pieces: allosteric activation of glycerol 3-phosphate dehydrogenase (NAD+) by phosphite dianion. Biochemistry (Mosc.) 47, 4575-4582.

Tung, W.L., and Chow, K.C. (1995). A modified medium for efficient electrotransformation of E. coli. Trends Genet. TIG 11, 128-129.

Turano, A., Furey, W., Pletcher, J., Sax, M., Pike, D., and Kluger, R. (1982). Synthesis and crystal structure of an analog of 2-(.alpha--lactyl) thiamin, racemic methyl 2-hydroxy-2(2-thiamin) ethylphosphonate chloride trihydrate. A conformation for a least-motion, maximum-overlap mechanism for thiamin catalysis. J. Am. Chem. Soc. 104, 3089-3095.

Turner, N.J. (2000). Applications of transketolases in organic synthesis. Curr. Opin. Biotechnol. 11, 527-531.

Veitch, N.J., Maugeri, D.A., Cazzulo, J.J., Lindqvist, Y., and Barrett, M.P. (2004). Transketolase from Leishmania mexicana has a dual subcellular localization. Biochem. J. 382, $759-767$.

Villafranca, J.J., and Axelrod, B. (1971). Heptulose synthesis from nonphosphorylated aldoses and ketoses by spinach transketolase. J. Biol. Chem. 246, 3126-3131.

Wang, J.J.-L., Martin, P.R., and Singleton, C.K. (1997). Aspartate 155 of human transketolase is essential for thiamine diphosphate-magnesium binding, and cofactor binding is required for dimer formation. Biochim. Biophys. Acta BBA - Protein Struct. Mol. Enzymol. $1341,165-172$.

Warshel, A., Papazyan, A., and Kollman, P.A. (1995). On low-barrier hydrogen bonds and enzyme catalysis. Science 269, 102-106.

Warshel, A., Sharma, P.K., Kato, M., Xiang, Y., Liu, H., and Olsson, M.H.M. (2006). Electrostatic basis for enzyme catalysis. Chem. Rev. 106, 3210-3235.

Washabaugh, M.W., and Jencks, W.P. (1988). Thiazolium C(2)-proton exchange: structurereactivity correlations and the $\mathrm{pKa}$ of thiamin $\mathrm{C}(2)-\mathrm{H}$ revisited. Biochemistry (Mosc.) 27, $5044-5053$.

Weber, K., Pringle, J.R., and Osborn, M. (1972). Measurement of molecular weights by electrophoresis on SDS-acrylamide gel. Methods Enzymol. 26, 3-27.

Wechsler, C. (2014). Intermediates in the carboligation of the ThDP-dependent pyruvate decarboxylase tailor-made enzyme catalysts for industrial applications. 
White, J.K., Handa, S., Vankayala, S.L., Merkler, D.J., and Woodcock, H.L. (2016). Thiamin Diphosphate Activation in 1-Deoxy-d-xylulose 5-Phosphate Synthase: Insights into the Mechanism and Underlying Intermolecular Interactions. J. Phys. Chem. B 120, 9922 9934.

Wikner, C., Nilsson, U., Meshalkina, L., Udekwu, C., Lindqvist, Y., and Schneider, G. (1997). Identification of Catalytically Important Residues in Yeast Transketolase. Biochemistry (Mosc.) 36, 15643-15649.

Wille, G., Meyer, D., Steinmetz, A., Hinze, E., Golbik, R., and Tittmann, K. (2006). The catalytic cycle of a thiamin diphosphate enzyme examined by cryocrystallography. Nat. Chem. Biol. 2, 324-328.

Williams, R.R. (1935). STRUCTURE OF VITAMIN B. J. Am. Chem. Soc. 57, 229-230.

Williams, R.R. (1936). STRUCTURE OF VITAMIN B1. J. Am. Chem. Soc. 58, 1063-1064. Williams, R.R., and Cline, J.K. (1936). SYNTHESIS OF VITAMIN B1. J. Am. Chem. Soc. $58,1504-1505$.

Xiang, S., Usunow, G., Lange, G., Busch, M., and Tong, L. (2007). Crystal Structure of 1Deoxy-d-xylulose 5-Phosphate Synthase, a Crucial Enzyme for Isoprenoids Biosynthesis. J. Biol. Chem. 282, 2676-2682.

Yamaguchi, S., Kamikubo, H., Kurihara, K., Kuroki, R., Niimura, N., Shimizu, N., Yamazaki, Y., and Kataoka, M. (2009). Low-barrier hydrogen bond in photoactive yellow protein. Proc. Natl. Acad. Sci. 106, 440-444.

Zahr, N.M., Kaufman, K.L., and Harper, C.G. (2011). Clinical and pathological features of alcohol-related brain damage. Nat. Rev. Neurol. 7, 284-294.

Zavitsas, A.A. (2003). The Relation between Bond Lengths and Dissociation Energies of Carbon-Carbon Bonds. J. Phys. Chem. A 107, 897-898.

Zhao, Y., Pan, X., Zhao, J., Wang, Y., Peng, Y., and Zhong, C. (2009). Decreased transketolase activity contributes to impaired hippocampal neurogenesis induced by thiamine deficiency. J. Neurochem. 111, 537-546.

Zimmermann, F.T., Schneider, A., Schörken, U., Sprenger, G.A., and Fessner, W.-D. (1999). Efficient multi-enzymatic synthesis of d-xylulose 5-phosphate. Tetrahedron Asymmetry 10, 1643-1646. 


\section{Curriculum Vitae}

\section{Personal information}

Name:

Gender:

Date of Birth:

E-mail:

Nationality:

\section{Educational background}

9/2005-7/2009

9/2009-7/2012

2/2013-present

\section{Teaching Experience}

2014, 2015, 2016

2014, 2015, 2016

\section{Shao-Bo Dai}

male

20/08/1986

Shaobo.Dai@biologie.uni-goettingen.de

Chinese
Bachelor studies of Life Science and Technology.

College of Life Sciences, Northwest University, Xi'an, China.

Bachelor Thesis: Chemo-enzymatic synthesis of 1-deoxy-Dxylulose 5-phosphate and 1-deoxy-D-xylulose.

under the supervision of Prof. Dr. Wen-Yun Gao

Master studies of Biochemistry and Molecular Biology.

College of Life Sciences, Northwest University, Xi'an, China.

Master Thesis: Studies on the catalytic mechanism of thiamine diphosphate dependent enzyme 1-deoxy-D-xylulose 5phosphate synthase.

under the supervision of Prof. Dr. Wen-Yun Gao

$\mathrm{PhD}$ studies under the supervision of Prof. Dr. Kai Tittmann.

Department of Molecular Enzymology at the University of Göttingen.

$\mathrm{PhD}$ Thesis: Mechanistic and structural characterization of thiamine diphosphate dependent enzyme transketolases from human and E.coli.

Supervision of practical course for B.Sc Biochemistry: enzyme kinetics

Supervision of practical course for M.Sc. Microbiology and Biochemistry: enzyme catalysis and biological chemistry

\section{Research Interest}

- Structure, mechanism and intermediate studies of enzyme catalysis

- Chemical and enzymatic syntheses of natural products 


\section{Skills and Interests}

Languages

Computer skills

Experimental skills

Hobbies
English: advanced in written and spoken,

German: basic,

Chinese: mother tongue.

Windows and Linux, Crystallographic software (XDS, MOSFLM, CCP4 suite, PHENIX suite, SHELX, COOT, Chimera, PYMOL), MestreNova, Origin, SigmaPlot, MS Office.

Protein crystallization, crystal fishing, data collection and processing, structure determination, refinement, and model building, Stopped-flow (single, sequential mixing), ITC, Tjump, CD-spectroscopy, UV-vis spectroscopy, HPLC, LCMS, GC and GC-MS, NMR.

Reading, playing badminton and basketball

\section{Conference Presentations and Posters}

- A chemo-enzymatic cascade for the one-pot syntheses of 1-deoxy-D-xylulose 5phosphate and 1-deoxy-D-xylulose. Presented at the 7th national conference of Chemical Biology. August 26-29th, 2011, Nanjing, China (Also as a poster)

- The role of phosphate in transketolase: capture of the central dihydroxyethyl thiamine diphosphate (DHEThDP) intermediate from non-phosphorylated substrates. EMBO conference, March 6-8th, 2017, Groningen, The Netherlands

\section{Publications}

- Dai S.B., Liao J., Tian J., Li H., Gao W.Y. A chemo-enzymatic cascade for the one-pot syntheses of 1-deoxy-D-xylulose 5-phosphate and 1-deoxy-D-xylulose. Tetrahedron Letters 53 (2012) 4809-4812.

- Li H., Dai S.B., Gao W.Y. Preparation of isotope labeled/unlabeled key intermediates in 2-methyl-D-erythritol 4-phosphate terpenoid biosynthetic pathway. Helvetica Chimica Acta 95 (2012) 683-715.

- Affaticati P.*, Dai S.B.*, Payongsri P., Tittmann K., Dalby P.-A. Structural Analysis of an Evolved Transketolase Reveals Divergent Binding Modes. Scientific Reports, 6 (2016) 35716 (*co-first author). 